\title{
Ligand and Substrate Effects on the Mechanism of Rhodium-Catalyzed Hydrogenation of Enamides
}

\author{
Patrick J. Donoghue, Paul Helquist and Olaf Wiest* \\ Department of Chemistry and Biochemistry, \\ University of Notre Dame, Notre Dame, Indiana, 46556-5670 \\ owiest@nd.edu \\ Supporting Information
}

\section{Contents}

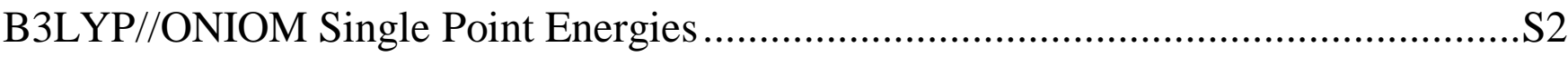

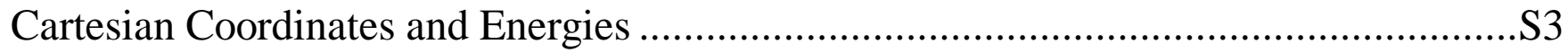

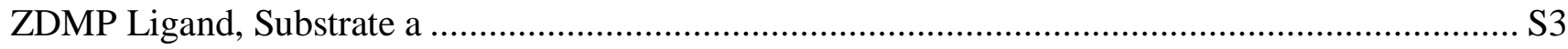

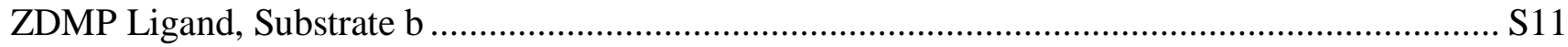

ZDMP Ligand, Substrate c ……………………………….............................................. S20

ZDMP Ligand, Substrate d .............................................................................................. S28

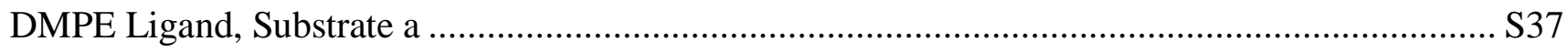

DuPHOS Ligand, Substrate a, Major Manifold …………………………………………........ S46

DuPHOS Ligand, Substrate a, Minor Manifold ……………………......................................... S59

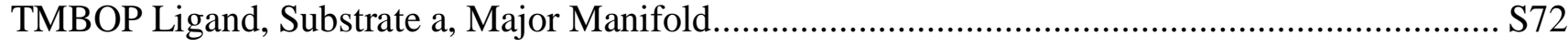

TMBOP Ligand, Substrate a, Minor Manifold …………………………………………............. S85

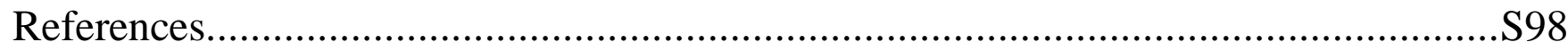


Supporting Information for Donoghue et al.: Ligand and Substrate Effects on the Mechanisms of the Rhodium...

\section{B3LYP//ONIOM Single Point Energies}

Energies shown are single point SCF energies at B3LYP/LACVP** at the optimized geometries reported by Landis and coworkers ${ }^{4 \mathrm{~b}}$ using ONIOM (B3LYP/LANL2DZ:HF/LANL2MB:UFF). Nomenclature is used as defined in reference $4 \mathrm{~b}$.

\begin{tabular}{|c|c|c|c|c|c|}
\hline$\cdots \cdots \cdots$ & $\begin{array}{l}\mathrm{SCF} \\
\mathrm{SCF}\end{array}$ & $\begin{array}{l}\text { Energy } \\
\text { Energy }\end{array}$ & $\begin{array}{l}= \\
=\end{array}$ & $\begin{array}{l}-1834.452076 \\
-1835.629919\end{array}$ & $\begin{array}{l}\text { lartree } \\
\text { lartree }\end{array}$ \\
\hline$\ldots \ldots \ldots$ & $\mathrm{SCF}$ & Energy & $=$ & -1835.629541 & 列 \\
\hline$D-A-t s \ldots \ldots \ldots$ & $\mathrm{SCF}$ & Energy & $=$ & -1835.61 & $r t$ \\
\hline ID-B-ts $\ldots \ldots \ldots$ & $\mathrm{SCF}$ & Energy & $=$ & -1835.596667 & +2 \\
\hline$D-C-t s \ldots \ldots \ldots$ & $\mathrm{SCF}$ & Energy & $=$ & -1835.628397 & $r t$ \\
\hline$I D-D-t s \ldots \ldots \ldots$ & $\mathrm{SCF}$ & Energy & $=$ & -1835.591472 & \\
\hline$\ldots \ldots \ldots$ & $\mathrm{SCF}$ & Energy & $=$ & -1835.614909 & 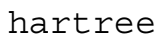 \\
\hline $\mathrm{OLH} 2-\mathrm{BD} \ldots \ldots \ldots \ldots$ & $\mathrm{SCF}$ & Energy & $=$ & -1835.601030 & \\
\hline$\ldots \ldots \ldots$ & $\mathrm{SCF}$ & Energy & $=$ & -1835.63 & 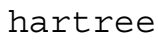 \\
\hline OLH2 -A-ts . . . . . . . & $\mathrm{SCF}$ & Energy & $=$ & -1835.606024 & \\
\hline $\mathrm{OLH} 2-\mathrm{B}-\mathrm{ts} \ldots \ldots \ldots$ & $\mathrm{SCF}$ & Energy & $=$ & -1835.602412 & \\
\hline MOLH2 $-C-t s \ldots \ldots \ldots$ & $\mathrm{SCF}$ & Energy & $=$ & -1835.613305 & \\
\hline 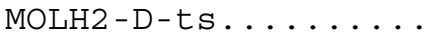 & $\mathrm{SCF}$ & Energy & $=$ & -1835.599625 & \\
\hline DIHY-A ........... & $\mathrm{SCF}$ & Energy & $=$ & -1835.608793 & \\
\hline$\ldots \ldots \ldots$ & $\mathrm{SCF}$ & Energy & $=$ & -1835.628272 & $\perp$ \\
\hline DIHY-C ........... & $\mathrm{SCF}$ & Energy & $=$ & -1835.616115 & \\
\hline$\ldots \ldots \ldots$ & $\mathrm{SCF}$ & Energy & $=$ & -1835.622273 & \\
\hline DIHY-Aa-ts ........ & $\mathrm{SCF}$ & Energy & $=$ & -1835.608624 & \\
\hline DIHY-Ba-ts .......... & $\mathrm{SCF}$ & Energy & $=$ & -1835.625768 & \\
\hline $\mathrm{DIHY}-\mathrm{Cb}-\mathrm{ts} \ldots \ldots \ldots$ & $\mathrm{SCF}$ & Energy & $=$ & -1835.594 & \\
\hline DIHY-Db-ts ......... & $\mathrm{SCF}$ & Energy & $=$ & -1835.608546 & \\
\hline ALHY-Ba ........... & SCF & Energy & $=$ & -1835.65 & \\
\hline ALHY-CDb . . . . . . . . & $\mathrm{SCF}$ & Energy & $=$ & -1835.639873 & \\
\hline PROD-A . . . . . . . & $\mathrm{SCF}$ & Energy & $=$ & -1835.67 & \\
\hline MIN .............. & $\mathrm{SCF}$ & Energy & $=$ & -1834.446065 & \\
\hline 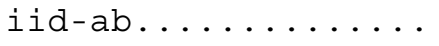 & $\mathrm{SCF}$ & Energy & $=$ & -1835 & \\
\hline$\ldots \ldots \ldots$ & $\mathrm{SCF}$ & Energy & $=$ & -1835.624484 & 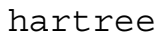 \\
\hline 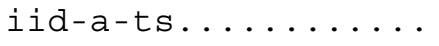 & $\mathrm{SCF}$ & Energy & $=$ & -1835.619566 & \\
\hline 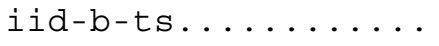 & $\mathrm{SCF}$ & Energy & $=$ & -1835.590655 & \\
\hline 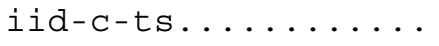 & $\mathrm{SCF}$ & Energy & $=$ & 5.623422 & \\
\hline 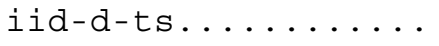 & $\mathrm{SCF}$ & Energy & $=$ & -1835.595787 & \\
\hline $\operatorname{molh} 2-a \ldots . . .$. & $\mathrm{SCF}$ & Energy & $=$ & .622360 & \\
\hline $\operatorname{molh} 2-\mathrm{bd} \ldots \ldots$ & $\mathrm{SCF}$ & Energy & $=$ & .605103 & \\
\hline $\operatorname{molh} 2-c \ldots \ldots \ldots$ & $\mathrm{SCF}$ & Energy & $=$ & -1835.626191 & \\
\hline $\operatorname{molh} 2-a-t s \ldots \ldots$. & $\mathrm{SCF}$ & Energy & $=$ & -1835.611297 & \\
\hline $\operatorname{molh} 2-\mathrm{b}-\mathrm{ts} \ldots \ldots \ldots$ & $\mathrm{SCF}$ & Energy & $=$ & 5.601651 & $2+$ \\
\hline $\operatorname{molh} 2-c-t s \ldots \ldots .$. & $\mathrm{SCF}$ & Energy & $=$ & -1835.608813 & \\
\hline $\operatorname{molh} 2-d-t s \ldots . .$. & $\mathrm{SCF}$ & Energy & $=$ & .600298 & h \\
\hline$\ldots \ldots \ldots$ & $\mathrm{SCF}$ & Energy & $=$ & -1835.615191 & \\
\hline dihy-b. & $\mathrm{SCF}$ & Energy & $=$ & -1835.626050 & $1+$ \\
\hline$\ldots \ldots \ldots \ldots$ & $\mathrm{SCF}$ & Energy & $=$ & -1835.609709 & \\
\hline dihy-d. & $\mathrm{SCF}$ & Energy & $=$ & -1835.623978 & ct \\
\hline dihy-aa-ts........ & $\mathrm{SCF}$ & Energy & $=$ & -1835.613268 & \\
\hline dihy-ba-ts.... & $\mathrm{SCF}$ & Energy & $=$ & -1835.624689 & ret \\
\hline dihy-cb-ts........ & $\mathrm{SCF}$ & Energy & $=$ & -1835.589081 & \\
\hline dihy-db-ts.... & $\mathrm{SCF}$ & Energy & $=$ & -1835.609704 & hart \\
\hline 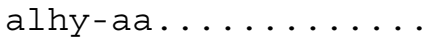 & $\mathrm{SCF}$ & Energy & $=$ & -1835.658809 & \\
\hline alhy-aa-trans.. & $\mathrm{SCF}$ & Energy & $=$ & -1835.633820 & \\
\hline alhy-ba. & $\mathrm{SCF}$ & Energy & - & 649229 & \\
\hline
\end{tabular}


Supporting Information for Donoghue et al.: Ligand and Substrate Effects on the Mechanisms of the Rhodium...

alhy-cdb.......................... Energy $=-1835.640113$ hartree

prod-a............SCF Energy $=-1835.677331$ hartree

\section{Cartesian Coordinates and Energies}

All coordinates shown are optimized at B3LYP/LACVP** and listed in angstroms. Energies shown are in hartrees. Total Enthalpies include ZPE and thermal corrections and are listed as $\mathrm{H}_{\text {tot }}$ in the Jaguar 5.5 output file. All negative frequencies are listed in $\mathrm{cm}^{-1}$.

$\mathrm{H}_{2}$

$\begin{array}{lll}\text { SCF Energy } & =-1.178539 & \text { hartree } \\ \text { Total Enthalpy }=-1.165051 & \text { hartree }\end{array}$
$\mathrm{H}$
$-0.37126$
0.00000
0.00000
$\mathrm{H}$
0.37126
0.00000
0.00000

\section{ZDMP Ligand, Substrate a}

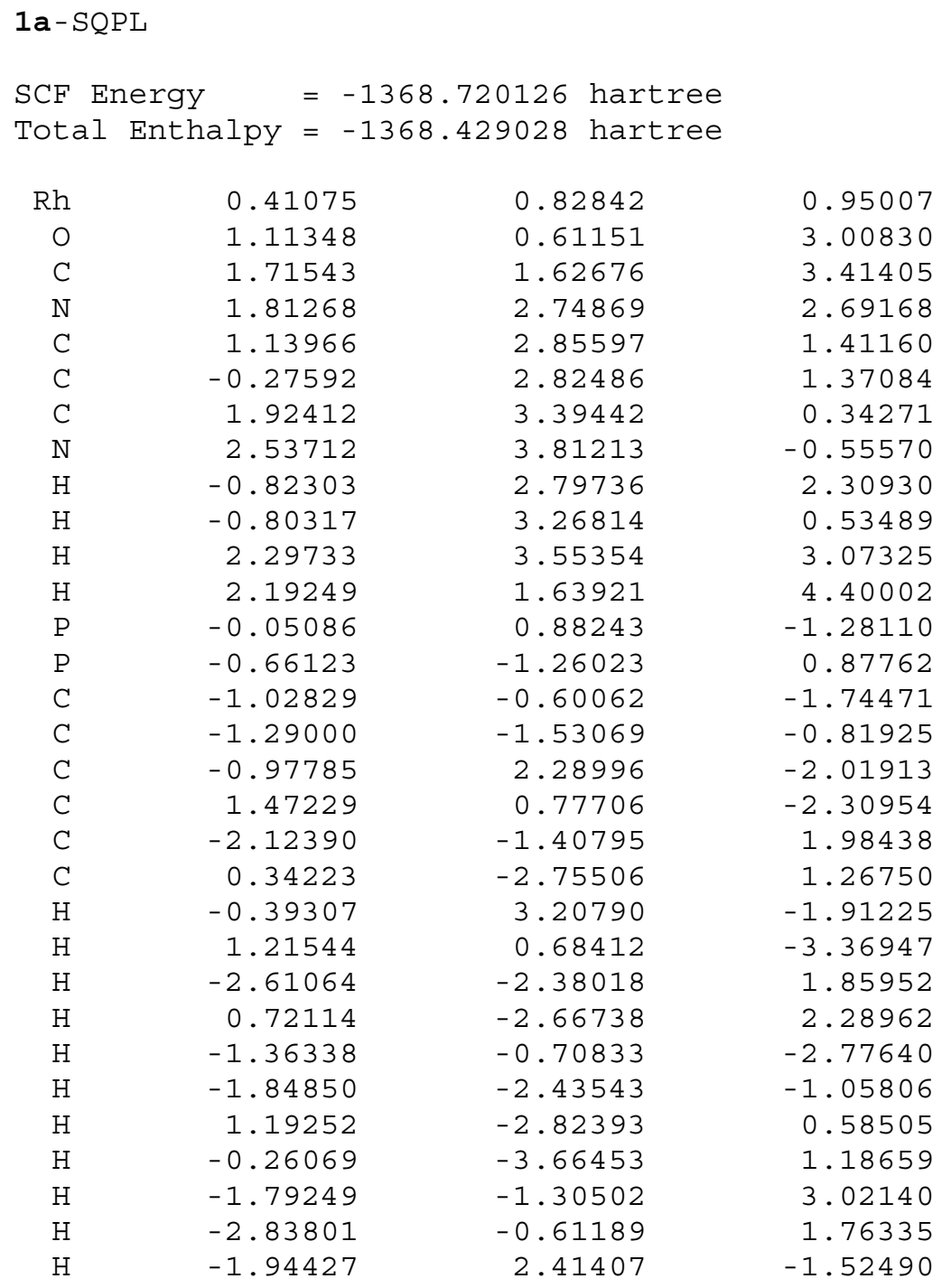


Supporting Information for Donoghue et al.: Ligand and Substrate Effects on the Mechanisms of the Rhodium...

$\begin{array}{rrrr}\mathrm{H} & -1.14419 & 2.11012 & -3.08541 \\ \mathrm{H} & 2.06427 & 1.68439 & -2.15836 \\ \mathrm{H} & 2.06645 & -0.08816 & -2.00646\end{array}$

$1 \mathrm{a}-\mathrm{MOLH}-\mathrm{A}$

$\begin{array}{lll}\text { SCF Energy } & =-1369.893203 & \text { hartree } \\ \text { Total Enthalpy } & =-1369.586013 \text { hartree }\end{array}$

\begin{tabular}{|c|c|c|c|}
\hline $\mathrm{Rh}$ & 0.12824 & 1.06375 & 0.95405 \\
\hline O & 0.33800 & 0.95333 & 3.12570 \\
\hline $\mathrm{C}$ & 1.53865 & 0.93441 & 3.46863 \\
\hline $\mathrm{N}$ & 2.53240 & 1.00891 & 2.58188 \\
\hline C & 2.23512 & 1.33489 & 1.18938 \\
\hline C & 1.64241 & 2.61623 & 0.92704 \\
\hline C & 3.11145 & 0.66390 & 0.27151 \\
\hline $\mathrm{N}$ & 3.81857 & 0.10463 & -0.46432 \\
\hline $\mathrm{H}$ & 1.54431 & 3.31023 & 1.75637 \\
\hline $\mathrm{H}$ & 1.79952 & 3.07846 & -0.04087 \\
\hline $\mathrm{H}$ & 3.49706 & 1.01305 & 2.89662 \\
\hline $\mathrm{H}$ & 1.81842 & 0.84607 & 4.52380 \\
\hline $\mathrm{P}$ & -0.13673 & 0.94219 & -1.33273 \\
\hline $\mathrm{P}$ & -0.61878 & -1.22792 & 0.92348 \\
\hline C & -0.72920 & -0.73399 & -1.77435 \\
\hline $\mathrm{C}$ & -0.94473 & -1.65245 & -0.82693 \\
\hline $\mathrm{H}$ & -1.70729 & 1.60360 & 1.16070 \\
\hline $\mathrm{H}$ & -1.30790 & 2.28970 & 1.11026 \\
\hline C & -1.45088 & 2.06620 & -1.96739 \\
\hline $\mathrm{C}$ & 1.26249 & 1.23635 & -2.48640 \\
\hline $\mathrm{C}$ & -2.15711 & -1.71793 & 1.81324 \\
\hline $\mathrm{C}$ & 0.60054 & -2.49987 & 1.47037 \\
\hline $\mathrm{H}$ & -1.14367 & 3.10492 & -1.81800 \\
\hline $\mathrm{H}$ & 0.91740 & 1.07722 & -3.51277 \\
\hline $\mathrm{H}$ & -2.38342 & -2.77842 & 1.66696 \\
\hline $\mathrm{H}$ & 0.78743 & -2.37580 & 2.54090 \\
\hline $\mathrm{H}$ & -0.90339 & -0.94976 & -2.82886 \\
\hline $\mathrm{H}$ & -1.30694 & -2.64841 & -1.08229 \\
\hline $\mathrm{H}$ & 1.54045 & -2.36570 & 0.93017 \\
\hline $\mathrm{H}$ & 0.22237 & -3.51181 & 1.29674 \\
\hline $\mathrm{H}$ & -2.02133 & -1.52746 & 2.88168 \\
\hline $\mathrm{H}$ & -2.99926 & -1.11734 & 1.46066 \\
\hline $\mathrm{H}$ & -2.38463 & 1.89348 & -1.42828 \\
\hline $\mathrm{H}$ & -1.61537 & 1.89512 & -3.03552 \\
\hline $\mathrm{H}$ & 1.61439 & 2.26730 & -2.39522 \\
\hline $\mathrm{H}$ & 2.09148 & 0.55993 & -2.2735 \\
\hline
\end{tabular}

$1 \mathrm{a}-\mathrm{MOLH}-\mathrm{C}$

SCF Energy = -1369.900104 hartree

Total Enthalpy $=-1369.592944$ hartree

$\begin{array}{rrrr}\text { Rh } & 0.68404 & -0.28896 & 0.98202 \\ \mathrm{O} & 0.70854 & -0.37239 & 3.17260 \\ \mathrm{C} & 1.87393 & -0.35156 & 3.62747 \\ \mathrm{~N} & 2.94452 & -0.18579 & 2.84796 \\ \mathrm{C} & 2.76009 & 0.13781 & 1.44400\end{array}$


Supporting Information for Donoghue et al.: Ligand and Substrate Effects on the Mechanisms of the Rhodium...

$\begin{array}{rrrr}\mathrm{C} & 2.01909 & 1.33345 & 1.12467 \\ \mathrm{C} & 3.74075 & -0.40829 & 0.55705 \\ \mathrm{~N} & 4.50786 & -0.84755 & -0.20220 \\ \mathrm{H} & 1.74621 & 1.99200 & 1.94570 \\ \mathrm{H} & 2.23218 & 1.84510 & 0.19343 \\ \mathrm{H} & 3.87192 & -0.16031 & 3.25742 \\ \mathrm{H} & 2.05601 & -0.47305 & 4.70014 \\ \mathrm{P} & 0.65526 & -0.32806 & -1.30980 \\ \mathrm{P} & -1.59312 & 0.34783 & 0.77213 \\ \mathrm{C} & -1.03176 & 0.08325 & -1.89722 \\ \mathrm{C} & -1.99208 & 0.38194 & -1.01503 \\ \mathrm{H} & 0.00282 & -2.19970 & 1.11745 \\ \mathrm{H} & 0.77510 & -2.27519 & 1.09002 \\ \mathrm{C} & 1.75211 & 0.79087 & -2.27192 \\ \mathrm{C} & 1.02951 & -1.98796 & -2.00740 \\ \mathrm{C} & -2.01022 & 2.03266 & 1.38940 \\ \mathrm{C} & -2.90166 & -0.71202 & 1.52013 \\ \mathrm{H} & 2.79705 & 0.57019 & -2.03799 \\ \mathrm{H} & 0.90618 & -1.98286 & -3.09477 \\ \mathrm{H} & -3.05337 & 2.28490 & 1.17621 \\ \mathrm{H} & -2.74335 & -0.75636 & 2.60145 \\ \mathrm{H} & -3.00095 & 0.63498 & -1.34114 \\ \mathrm{H} & -1.22046 & 0.08459 & -2.97098 \\ \mathrm{H} & 1.53674 & 1.83611 & -2.03995 \\ \mathrm{H} & 1.59049 & 0.62682 & -3.34171 \\ \mathrm{H} & 2.06585 & -2.24341 & -1.76744 \\ \mathrm{H} & 0.36542 & -2.73884 & -1.57406 \\ \mathrm{H} & -2.83646 & -1.72616 & 1.11812 \\ \mathrm{H} & -3.89997 & -0.30979 & 1.32309 \\ \mathrm{H} & -1.85267 & 2.05880 & 0.92301 \\ \mathrm{H} & -1.35454 & 2.77117 & \end{array}$

$1 a-M O L H-t s-A$

SCF Energy $=-1369.883201$ hartree
Total Enthalpy $=-1369.577632$ hartree
Negative Frequencies: $-864.27 \mathrm{~cm}^{-1}$

$\begin{array}{rrrr}\mathrm{Rh} & 0.00000 & 0.00000 & 0.00000 \\ \mathrm{O} & 2.19570 & 0.00000 & 0.00000 \\ \mathrm{C} & 2.69164 & 1.14328 & 0.00000 \\ \mathrm{~N} & 1.94950 & 2.25741 & 0.03316 \\ \mathrm{C} & 0.52131 & 2.17977 & 0.27447 \\ \mathrm{C} & 0.08084 & 1.62922 & 1.50033 \\ \mathrm{C} & -0.26925 & 3.06778 & -0.52204 \\ \mathrm{~N} & -0.90967 & 3.77732 & -1.18801 \\ \mathrm{H} & 0.81947 & 1.38540 & 2.25566 \\ \mathrm{H} & -0.91003 & 1.86456 & 1.86687 \\ \mathrm{H} & 2.40591 & 3.16320 & 0.05298 \\ \mathrm{H} & 3.77776 & 1.28062 & -0.04001 \\ \mathrm{P} & -2.28164 & -0.11917 & -0.17981 \\ \mathrm{P} & -0.01953 & -0.18318 & -2.44125 \\ \mathrm{C} & -2.71955 & -0.37112 & -1.93856 \\ \mathrm{C} & -1.78008 & -0.40295 & -2.88906 \\ \mathrm{H} & -0.05850 & -1.59416 & 0.17249 \\ \mathrm{H} & -0.08943 & -1.00979 & 1.25075 \\ \mathrm{C} & -3.07342 & -1.52351 & 0.70036\end{array}$


Supporting Information for Donoghue et al.: Ligand and Substrate Effects on the Mechanisms of the Rhodium...

$\begin{array}{rrrr}\mathrm{C} & -3.27687 & 1.34000 & 0.32784 \\ \mathrm{C} & 0.83634 & -1.65381 & -3.14529 \\ \mathrm{C} & 0.53838 & 1.19738 & -3.53105 \\ \mathrm{H} & -2.92293 & -1.40621 & 1.77681 \\ \mathrm{H} & -4.32466 & 1.17347 & 0.05915 \\ \mathrm{H} & 0.69350 & -1.71703 & -4.22806 \\ \mathrm{H} & 1.61557 & 1.33992 & -3.40553 \\ \mathrm{H} & -3.77626 & -0.49080 & -2.17955 \\ \mathrm{H} & -2.05052 & -0.54773 & -3.93517 \\ \mathrm{H} & 0.02490 & 2.12119 & -3.25530 \\ \mathrm{H} & 0.33772 & 0.96935 & -4.58236 \\ \mathrm{H} & 1.90552 & -1.57340 & -2.92969 \\ \mathrm{H} & 0.45812 & -2.56188 & -2.67087 \\ \mathrm{H} & -2.61746 & -2.46352 & 0.38280 \\ \mathrm{H} & -4.14670 & -1.54859 & 0.48939 \\ \mathrm{H} & -3.21675 & 1.47456 & 1.41113 \\ \mathrm{H} & -2.91870 & 2.24167 & -0.17280\end{array}$

1a-MOLH-ts-C

SCF Energy $=-1369.881949$ hartree
Total Enthalpy $=-1369.576467$ hartree
Negative Frequencies: $-843.42 \mathrm{~cm}^{-1}$

$\begin{array}{rrrr}\text { Rh } & 0.00000 & 0.00000 & 0.00000 \\ \mathrm{O} & 2.19198 & 0.00000 & 0.00000 \\ \mathrm{C} & 2.66642 & 1.15343 & 0.00000 \\ \mathrm{~N} & 1.90832 & 2.24600 & 0.14021 \\ \mathrm{C} & 0.50347 & 2.12518 & 0.47792 \\ \mathrm{C} & 0.15325 & 1.47621 & 1.68368 \\ \mathrm{C} & -0.34728 & 3.09688 & -0.14669 \\ \mathrm{~N} & -1.04738 & 3.89177 & -0.62858 \\ \mathrm{H} & 0.94668 & 1.17945 & 2.36068 \\ \mathrm{H} & -0.81169 & 1.67626 & 2.13205 \\ \mathrm{H} & 2.33285 & 3.16644 & 0.10922 \\ \mathrm{H} & 3.74272 & 1.31490 & -0.12084 \\ \mathrm{P} & -2.28136 & -0.08810 & -0.19604 \\ \mathrm{P} & -0.25365 & -1.92683 & 1.44133 \\ \mathrm{C} & -2.87954 & -1.57259 & 0.69691 \\ \mathrm{C} & -2.03564 & -2.34488 & 1.39062 \\ \mathrm{H} & 0.01770 & -0.97305 & -1.26993 \\ \mathrm{H} & 0.06902 & 0.28267 & -1.58425 \\ \mathrm{C} & -3.28138 & 1.28915 & 0.49660 \\ \mathrm{C} & -2.91278 & -0.27174 & -1.90944 \\ \mathrm{C} & 0.14649 & -1.85856 & 3.24065 \\ \mathrm{C} & 0.61438 & -3.44834 & 0.87739 \\ \mathrm{H} & -3.02748 & 2.22338 & -0.01033 \\ \mathrm{H} & -3.99859 & -0.40574 & -1.90482 \\ \mathrm{H} & -0.11178 & -2.79849 & 3.73794 \\ \mathrm{H} & 1.69255 & -3.26696 & 0.90791 \\ \mathrm{H} & -2.39457 & -3.22115 & 1.93081 \\ \mathrm{H} & -3.94552 & -1.79687 & 0.64903 \\ \mathrm{H} & -3.09597 & 1.39911 & 1.56718 \\ \mathrm{H} & -4.34409 & 1.07679 & 0.34407 \\ \mathrm{H} & -2.66544 & 0.62956 & -2.47684 \\ \mathrm{H} & -2.43943 & -1.13084 & -2.38894 \\ \mathrm{H} & 0.33213 & -3.67173 & -0.15355\end{array}$


Supporting Information for Donoghue et al.: Ligand and Substrate Effects on the Mechanisms of the Rhodium...

$\begin{array}{rrrr}\mathrm{H} & 0.37664 & -4.30241 & 1.51847 \\ \mathrm{H} & 1.21985 & -1.68389 & 3.35892 \\ \mathrm{H} & -0.39691 & -1.03901 & 3.71610\end{array}$

$1 a-D I H Y-A$

SCF Energy = $\quad-1369.889515$ hartree

Total Enthalpy $=-1369.581346$ hartree

$\begin{array}{rrrr}\mathrm{Rh} & -0.12552 & 1.07063 & 1.02682 \\ \mathrm{O} & -0.32087 & 0.88134 & 3.22608 \\ \mathrm{C} & 0.74533 & 0.70339 & 3.83615 \\ \mathrm{~N} & 1.95157 & 0.70904 & 3.23826 \\ \mathrm{C} & 2.10352 & 1.10774 & 1.86115 \\ \mathrm{C} & 1.72082 & 2.38677 & 1.47161 \\ \mathrm{C} & 2.95433 & 0.28535 & 1.05437 \\ \mathrm{~N} & 3.65378 & -0.37184 & 0.39481 \\ \mathrm{H} & 1.40523 & 3.10052 & 2.22280 \\ \mathrm{H} & 2.05444 & 2.78823 & 0.52408 \\ \mathrm{H} & 2.78329 & 0.55624 & 3.79789 \\ \mathrm{H} & 0.75058 & 0.51603 & 4.91612 \\ \mathrm{P} & -0.13069 & 1.15826 & -1.24478 \\ \mathrm{P} & -0.33232 & -1.36223 & 0.75122 \\ \mathrm{C} & -0.41646 & -0.53374 & -1.88165 \\ \mathrm{C} & -0.49859 & -1.58422 & -1.05822 \\ \mathrm{H} & -1.66167 & 1.04492 & 0.80836 \\ \mathrm{H} & -0.58993 & 2.58260 & 0.99777 \\ \mathrm{C} & -1.43007 & 2.18288 & -2.03625 \\ \mathrm{C} & 1.42803 & 1.68830 & -2.06458 \\ \mathrm{C} & -1.89509 & -2.04560 & 1.44863 \\ \mathrm{C} & 0.92670 & -2.62150 & 1.23481 \\ \mathrm{H} & -1.27209 & 3.23069 & -1.76873 \\ \mathrm{H} & 1.34262 & 1.54555 & -3.14627 \\ \mathrm{H} & -2.02920 & -3.09474 & 1.16996 \\ \mathrm{H} & 1.01221 & -2.64092 & 2.32517 \\ \mathrm{H} & -0.51384 & -0.65359 & -2.96118 \\ \mathrm{H} & -0.66057 & -2.58715 & -1.45359 \\ \mathrm{H} & 1.89937 & -2.36922 & 0.80801 \\ \mathrm{H} & 0.62554 & -3.61623 & 0.89240 \\ \mathrm{H} & -1.85813 & -1.96647 & 2.53879 \\ \mathrm{H} & -2.74431 & -1.45911 & 1.09193 \\ \mathrm{H} & -2.41239 & 1.87613 & -1.67170 \\ \mathrm{H} & -1.39119 & 2.07802 & -3.12457 \\ \mathrm{H} & 1.60737 & 2.74939 & -1.87004 \\ \mathrm{H} & 2.27081 & 1.10046 & -1.69385\end{array}$

$1 a-D I H Y-C$

SCF Energy = $\quad-1369.887197$ hartree Total Enthalpy $=-1369.579184$ hartree

$\begin{array}{rrrr}\text { Rh } & 0.54355 & -0.62746 & 0.98074 \\ \mathrm{O} & 0.50009 & -0.74261 & 3.18465 \\ \mathrm{C} & 1.62089 & -0.69661 & 3.71363 \\ \mathrm{~N} & 2.74293 & -0.38655 & 3.03934 \\ \mathrm{C} & 2.70819 & 0.04581 & 1.66463 \\ \mathrm{C} & 2.03247 & 1.20166 & 1.31592\end{array}$


Supporting Information for Donoghue et al.: Ligand and Substrate Effects on the Mechanisms of the Rhodium...

$\begin{array}{rrrr}\mathrm{C} & 3.70980 & -0.54015 & 0.81722 \\ \mathrm{~N} & 4.55087 & -0.98085 & 0.14639 \\ \mathrm{H} & 1.57635 & 1.80859 & 2.08778 \\ \mathrm{H} & 2.19300 & 1.65326 & 0.34540 \\ \mathrm{H} & 3.64005 & -0.43401 & 3.50920 \\ \mathrm{H} & 1.75156 & -0.91277 & 4.77991 \\ \mathrm{P} & 0.48471 & -0.84149 & -1.28253 \\ \mathrm{P} & -1.27716 & 0.98720 & 0.70466 \\ \mathrm{C} & -0.91800 & 0.15881 & -1.90978 \\ \mathrm{C} & -1.65246 & 0.91862 & -1.08853 \\ \mathrm{H} & -0.61319 & -1.65380 & 0.87947 \\ \mathrm{H} & 1.28576 & -2.02683 & 0.97406 \\ \mathrm{C} & 1.93599 & -0.25057 & -2.24401 \\ \mathrm{C} & 0.18297 & -2.53191 & -1.92710 \\ \mathrm{C} & -1.17362 & 2.79889 & 1.05270 \\ \mathrm{C} & -2.84985 & 0.50192 & 1.53161 \\ \mathrm{H} & 2.82326 & -0.82222 & -1.96062 \\ \mathrm{H} & 0.06573 & -2.51373 & -3.01458 \\ \mathrm{H} & -2.09006 & 3.31023 & 0.74268 \\ \mathrm{H} & -2.71269 & 0.57054 & 2.61456 \\ \mathrm{H} & -2.48202 & 1.51117 & -1.47533 \\ \mathrm{H} & -1.13481 & 0.11250 & -2.97750 \\ \mathrm{H} & 2.11981 & 0.80847 & -2.04940 \\ \mathrm{H} & 1.74864 & -0.38483 & -3.31391 \\ \mathrm{H} & 1.03381 & -3.16617 & -1.66583 \\ \mathrm{H} & -0.71518 & -2.94695 & -1.46578 \\ \mathrm{H} & -3.09103 & -0.53323 & 1.28155 \\ \mathrm{H} & -3.67517 & 1.15480 & 1.23308 \\ \mathrm{H} & -1.03857 & 2.95200 & 2.12755 \\ \mathrm{H} & -0.32515 & 3.24080 & 0.52486\end{array}$

$1 a-D I H Y-t s-A$

SCF Energy $=-1369.882959$ hartree Total Enthalpy $=-1369.576442$ hartree Negative Frequencies: $-705.66 \mathrm{~cm}^{-1}$

$\begin{array}{rrrr}\text { Rh } & 0.00000 & 0.00000 & 0.00000 \\ \mathrm{O} & 2.21545 & 0.00000 & 0.00000 \\ \mathrm{C} & 2.73309 & 1.13277 & 0.00000 \\ \mathrm{~N} & 2.01767 & 2.26490 & 0.08440 \\ \mathrm{C} & 0.59698 & 2.21987 & 0.33157 \\ \mathrm{C} & 0.15305 & 1.68539 & 1.57295 \\ \mathrm{C} & -0.21069 & 3.06615 & -0.48388 \\ \mathrm{~N} & -0.86488 & 3.74821 & -1.16744 \\ \mathrm{H} & 0.90083 & 1.54319 & 2.34735 \\ \mathrm{H} & -0.83249 & 1.96054 & 1.93035 \\ \mathrm{H} & 2.49536 & 3.15960 & 0.07215 \\ \mathrm{H} & 3.81964 & 1.25228 & -0.07533 \\ \mathrm{P} & -2.25956 & -0.25645 & -0.12859 \\ \mathrm{P} & -0.00237 & -0.32266 & -2.35354 \\ \mathrm{C} & -2.67705 & -0.70657 & -1.85222 \\ \mathrm{C} & -1.72815 & -0.73909 & -2.79364 \\ \mathrm{H} & -0.09357 & -1.55694 & 0.10722 \\ \mathrm{H} & -0.09359 & 0.01786 & 1.63274 \\ \mathrm{C} & -3.00661 & -1.57367 & 0.90689 \\ \mathrm{C} & -3.28672 & 1.23021 & 0.21703\end{array}$


Supporting Information for Donoghue et al.: Ligand and Substrate Effects on the Mechanisms of the Rhodium...

$\begin{array}{lrrr}\mathrm{C} & 1.01724 & -1.73464 & -2.93722 \\ \mathrm{C} & 0.43615 & 1.06130 & -3.48666 \\ \mathrm{H} & -2.84019 & -1.33770 & 1.96131 \\ \mathrm{H} & -4.33343 & 1.02255 & -0.02642 \\ \mathrm{H} & 0.90834 & -1.88364 & -4.01529 \\ \mathrm{H} & 1.50116 & 1.28641 & -3.37889 \\ \mathrm{H} & -3.71766 & -0.93483 & -2.08286 \\ \mathrm{H} & -1.96753 & -0.99439 & -3.82578 \\ \mathrm{H} & -0.14024 & 1.95239 & -3.22659 \\ \mathrm{H} & 0.24031 & 0.78899 & -4.52822 \\ \mathrm{H} & 2.06539 & -1.52715 & -2.70541 \\ \mathrm{H} & 0.72202 & -2.64234 & -2.40689 \\ \mathrm{H} & -2.52705 & -2.52932 & 0.68662 \\ \mathrm{H} & -4.08230 & -1.64650 & 0.72081 \\ \mathrm{H} & -3.22520 & 1.48420 & 1.27920 \\ \mathrm{H} & -2.93860 & 2.07850 & -0.37650\end{array}$

$1 a-D I H Y-t s-C$

SCF Energy $=-1369.860352$ hartree

Total Enthalpy $=-1369.555052$ hartree Negative Frequencies: $-707.06 \mathrm{~cm}^{-1}$

$\begin{array}{rrrr}\text { Rh } & 0.00000 & 0.00000 & 0.00000 \\ \mathrm{O} & 2.23562 & 0.00000 & 0.00000 \\ \mathrm{C} & 2.75109 & 1.12925 & 0.00000 \\ \mathrm{~N} & 2.04457 & 2.26426 & 0.14345 \\ \mathrm{C} & 0.61884 & 2.20255 & 0.44280 \\ \mathrm{C} & 0.24787 & 1.51303 & 1.65518 \\ \mathrm{C} & -0.09403 & 3.42427 & 0.10129 \\ \mathrm{~N} & -0.69123 & 4.39016 & -0.14079 \\ \mathrm{H} & 1.05494 & 1.21055 & 2.31228 \\ \mathrm{H} & -0.67778 & 1.80452 & 2.13643 \\ \mathrm{H} & 2.50636 & 3.16533 & 0.11946 \\ \mathrm{H} & 3.83167 & 1.25677 & -0.13053 \\ \mathrm{P} & -2.23260 & -0.06573 & -0.38091 \\ \mathrm{P} & -0.28636 & -2.01960 & 1.09894 \\ \mathrm{C} & -2.86562 & -1.66575 & 0.24267 \\ \mathrm{C} & -2.03649 & -2.50434 & 0.87335 \\ \mathrm{H} & -0.00276 & -0.78091 & -1.38790 \\ \mathrm{H} & 0.19693 & 1.50138 & -0.76806 \\ \mathrm{C} & -3.24238 & 1.21098 & 0.47727 \\ \mathrm{C} & -2.78524 & 0.03458 & -2.12715 \\ \mathrm{C} & 0.00369 & -2.14290 & 2.91237 \\ \mathrm{C} & 0.71632 & -3.38250 & 0.38726 \\ \mathrm{H} & -2.95161 & 2.20633 & 0.12868 \\ \mathrm{H} & -3.87399 & -0.05316 & -2.19328 \\ \mathrm{H} & -0.22817 & -3.14827 & 3.27751 \\ \mathrm{H} & 1.77508 & -3.14219 & 0.51864 \\ \mathrm{H} & -2.38196 & -3.46167 & 1.26281 \\ \mathrm{H} & -3.91761 & -1.90588 & 0.08965 \\ \mathrm{H} & -3.08557 & 1.15175 & 1.55638 \\ \mathrm{H} & -4.30458 & 1.06029 & 0.26089 \\ \mathrm{H} & -2.47806 & 0.99840 & -2.54231 \\ \mathrm{H} & -2.31069 & -0.75930 & -2.70657 \\ \mathrm{H} & 0.51086 & -3.46417 & -0.68179 \\ \mathrm{H} & 0.49788 & -4.33285 & 0.88327 \\ & & & \end{array}$


Supporting Information for Donoghue et al.: Ligand and Substrate Effects on the Mechanisms of the Rhodium...

$\begin{array}{rrrr}\mathrm{H} & 1.05559 & -1.92639 & 3.11877 \\ \mathrm{H} & -0.61490 & -1.41281 & 3.43843\end{array}$

$1 \mathrm{a}-\mathrm{ALHY}-\mathrm{A}$

SCF Energy = $\quad-1369.927139$ hartree

Total Enthalpy $=-1369.615932$ hartree

$\begin{array}{rrrr}\mathrm{Rh} & 0.79037 & -0.09009 & 0.91396 \\ \mathrm{O} & 0.86679 & -0.16804 & 3.06229 \\ \mathrm{C} & 2.04365 & -0.22774 & 3.48781 \\ \mathrm{~N} & 3.09550 & -0.28431 & 2.67383 \\ \mathrm{C} & 2.90630 & -0.43865 & 1.22850 \\ \mathrm{C} & 4.06838 & 0.14237 & 0.44368 \\ \mathrm{C} & 2.50670 & -1.80081 & 0.91351 \\ \mathrm{~N} & 1.91094 & -2.77977 & 0.66238 \\ \mathrm{H} & 5.03026 & -0.27806 & 0.76844 \\ \mathrm{H} & 3.96683 & -0.07933 & -0.62085 \\ \mathrm{H} & 4.02570 & -0.37203 & 3.07249 \\ \mathrm{H} & 2.24112 & -0.23317 & 4.56444 \\ \mathrm{P} & 0.67892 & 0.18470 & -1.35687 \\ \mathrm{P} & -1.57760 & -0.01514 & 0.83724 \\ \mathrm{C} & -1.08036 & 0.39608 & -1.82610 \\ \mathrm{C} & -2.04344 & 0.31684 & -0.90068 \\ \mathrm{H} & 0.87681 & 1.42879 & 0.96592 \\ \mathrm{H} & 4.10652 & 1.22669 & 0.57274 \\ \mathrm{C} & 1.51644 & 1.64795 & -2.08849 \\ \mathrm{C} & 1.25155 & -1.23753 & -2.37266 \\ \mathrm{C} & -2.38924 & 1.29998 & 1.83154 \\ \mathrm{C} & -2.48450 & -1.55363 & 1.28646 \\ \mathrm{H} & 2.59282 & 1.58096 & -1.91258 \\ \mathrm{H} & 0.99301 & -1.07842 & -3.42386 \\ \mathrm{H} & -3.47383 & 1.29100 & 1.68978 \\ \mathrm{H} & -2.29721 & -1.77764 & 2.34033 \\ \mathrm{H} & -3.09357 & 0.43236 & -1.16867 \\ \mathrm{H} & -1.31296 & 0.57780 & -2.87563 \\ \mathrm{H} & 1.14188 & 2.55942 & -1.61761 \\ \mathrm{H} & 1.33519 & 1.69407 & -3.16645 \\ \mathrm{H} & 2.33594 & -1.34259 & -2.28619 \\ \mathrm{H} & 0.78917 & -2.15895 & -2.01343 \\ \mathrm{H} & -2.11905 & -2.38865 & 0.68433 \\ \mathrm{H} & -3.56124 & -1.43554 & 1.13206 \\ \mathrm{H} & -2.16444 & 1.12625 & 2.88755 \\ \mathrm{H} & -1.99112 & 2.27674 & 1.54802\end{array}$

$1 \mathrm{a}-\mathrm{ALHY}-\mathrm{C}$

SCF Energy = $\quad-1369.906807$ hartree

Total Enthalpy $=-1369.595118$ hartree

$\begin{array}{rrrr}\mathrm{Rh} & 0.63974 & 0.15634 & 0.79776 \\ \mathrm{O} & 0.64048 & 0.15086 & 2.94872 \\ \mathrm{C} & 1.63593 & 0.26192 & 3.69365 \\ \mathrm{~N} & 2.91418 & 0.14714 & 3.34255 \\ \mathrm{C} & 3.41791 & -0.21570 & 1.99656 \\ \mathrm{C} & 2.70853 & 0.52849 & 0.85126 \\ \mathrm{C} & 4.86454 & 0.06589 & 1.99428\end{array}$


Supporting Information for Donoghue et al.: Ligand and Substrate Effects on the Mechanisms of the Rhodium...

$\begin{array}{rrrr}\mathrm{N} & 6.00158 & 0.29565 & 1.96746 \\ \mathrm{H} & 2.83479 & 1.61245 & 0.99209 \\ \mathrm{H} & 3.23161 & 0.25475 & -0.06822 \\ \mathrm{H} & 3.60030 & 0.23460 & 4.08272 \\ \mathrm{H} & 1.47700 & 0.47661 & 4.75778 \\ \mathrm{P} & -1.74619 & -0.33430 & 0.68514 \\ \mathrm{P} & 0.52819 & 0.32056 & -1.46450 \\ \mathrm{C} & -2.14010 & -0.33050 & -1.10696 \\ \mathrm{C} & -1.18380 & -0.05269 & -2.00235 \\ \mathrm{H} & 0.95995 & -1.29407 & 0.53364 \\ \mathrm{H} & 3.29723 & -1.29901 & 1.86402 \\ \mathrm{C} & -2.31066 & -1.98645 & 1.27699 \\ \mathrm{C} & -2.98490 & 0.82180 & 1.41751 \\ \mathrm{C} & 1.58153 & -0.78443 & -2.48602 \\ \mathrm{C} & 0.87251 & 2.00763 & -2.11627 \\ \mathrm{H} & -2.16790 & -2.03719 & 2.36022 \\ \mathrm{H} & -4.00761 & 0.51785 & 1.17536 \\ \mathrm{H} & 1.37916 & -0.63075 & -3.55008 \\ \mathrm{H} & 1.91764 & 2.26486 & -1.92298 \\ \mathrm{H} & -3.15663 & -0.55046 & -1.43412 \\ \mathrm{H} & -1.39713 & -0.04090 & -3.07162 \\ \mathrm{H} & 0.23152 & 2.73968 & -1.61868 \\ \mathrm{H} & 0.69030 & 2.04904 & -3.19463 \\ \mathrm{H} & 2.63551 & -0.56847 & -2.29310 \\ \mathrm{H} & 1.38415 & -1.82702 & -2.22756 \\ \mathrm{H} & -1.71024 & -2.77180 & 0.81226 \\ \mathrm{H} & -3.36740 & -2.15452 & 1.04969 \\ \mathrm{H} & -2.86363 & 0.82355 & 2.50450 \\ \mathrm{H} & -2.81578 & 1.83648 & 1.04856\end{array}$

\section{ZDMP Ligand, Substrate b}

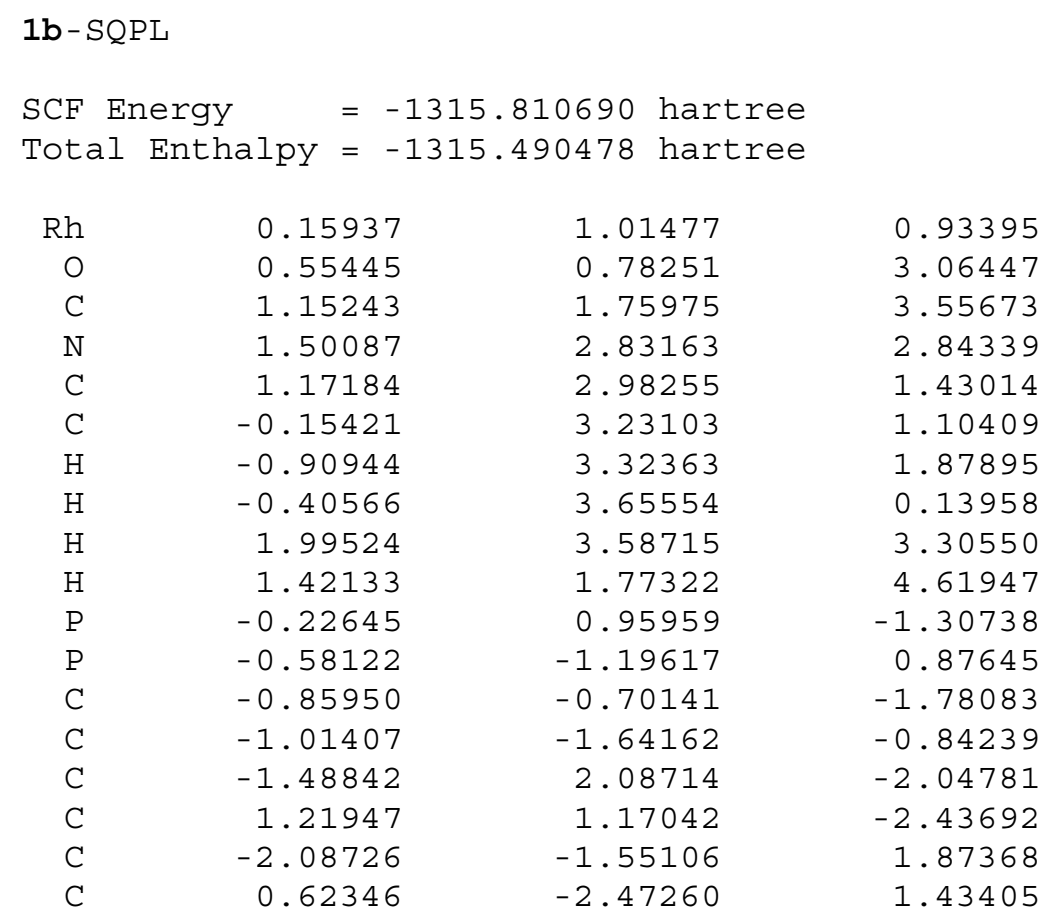


Supporting Information for Donoghue et al.: Ligand and Substrate Effects on the Mechanisms of the Rhodium...

$\begin{array}{rrrr}\mathrm{H} & -1.14251 & 3.12344 & -2.00572 \\ \mathrm{H} & 0.93264 & 0.97331 & -3.47464 \\ \mathrm{H} & -2.38462 & -2.59992 & 1.77803 \\ \mathrm{H} & 0.89943 & -2.25911 & 2.47027 \\ \mathrm{H} & -1.09750 & -0.89247 & -2.82762 \\ \mathrm{H} & -1.38414 & -2.63836 & -1.08104 \\ \mathrm{H} & 1.52348 & -2.43047 & 0.81676 \\ \mathrm{H} & 0.18961 & -3.47554 & 1.37725 \\ \mathrm{H} & -1.86994 & -1.33355 & 2.92290 \\ \mathrm{H} & -2.90780 & -0.90870 & 1.54692 \\ \mathrm{H} & -2.42543 & 2.00814 & -1.49202 \\ \mathrm{H} & -1.66788 & 1.82722 & -3.09551 \\ \mathrm{H} & 1.59805 & 2.19353 & -2.36853 \\ \mathrm{H} & 2.01736 & 0.48352 & -2.14668 \\ \mathrm{H} & 2.06510 & 3.52738 & -0.45260 \\ \mathrm{H} & 2.85393 & 4.23925 & 0.95651 \\ \mathrm{H} & 3.10960 & 2.52845 & 0.58408 \\ \mathrm{C} & 2.36524 & 3.33031 & 0.57781\end{array}$

1b-MOLH - A

SCF Energy = $\quad-1316.982550$ hartree

Total Enthalpy $=-1316.646779$ hartree

\begin{tabular}{|c|c|c|c|}
\hline $\mathrm{Rh}$ & 0.17222 & 1.00376 & 0.94416 \\
\hline O & 0.38263 & 0.87929 & 3.10790 \\
\hline $\mathrm{C}$ & 1.58415 & 0.92835 & 3.44839 \\
\hline $\mathrm{N}$ & 2.56858 & 1.06561 & 2.56289 \\
\hline C & 2.29892 & 1.41199 & 1.16508 \\
\hline C & 1.61109 & 2.64015 & 0.95718 \\
\hline $\mathrm{H}$ & 1.40926 & 3.29553 & 1.79959 \\
\hline $\mathrm{H}$ & 1.72019 & 3.14365 & 0.00205 \\
\hline $\mathrm{H}$ & 3.52764 & 1.10191 & 2.89288 \\
\hline $\mathrm{H}$ & 1.86688 & 0.84512 & 4.50366 \\
\hline $\mathrm{P}$ & -0.14889 & 0.91131 & -1.32158 \\
\hline $\mathrm{P}$ & -0.67043 & -1.23475 & 0.91693 \\
\hline $\mathrm{C}$ & -0.76086 & -0.75700 & -1.78170 \\
\hline $\mathrm{C}$ & -0.98272 & -1.67495 & -0.83445 \\
\hline $\mathrm{H}$ & -1.75952 & 1.62986 & 1.27523 \\
\hline $\mathrm{H}$ & -1.33886 & 2.27839 & 1.19499 \\
\hline $\mathrm{C}$ & -1.48338 & 2.04175 & -1.91145 \\
\hline $\mathrm{C}$ & 1.18958 & 1.21249 & -2.5516 \\
\hline $\mathrm{C}$ & -2.26539 & -1.63337 & 1.7583 \\
\hline $\mathrm{C}$ & 0.44208 & -2.57230 & 1.5356 \\
\hline $\mathrm{H}$ & -1.16609 & 3.07952 & -1.77618 \\
\hline $\mathrm{H}$ & 0.78072 & 1.13853 & -3.56388 \\
\hline $\mathrm{H}$ & -2.54705 & -2.68107 & 1.61509 \\
\hline $\mathrm{H}$ & 0.60998 & -2.42031 & 2.6057 \\
\hline $\mathrm{H}$ & -0.94793 & -0.96656 & -2.8352 \\
\hline $\mathrm{H}$ & -1.36211 & -2.66526 & -1.0871 \\
\hline $\mathrm{H}$ & 1.40371 & -2.52399 & 1.02002 \\
\hline $\mathrm{H}$ & -0.00131 & -3.56184 & 1.38750 \\
\hline $\mathrm{H}$ & -2.15649 & -1.43767 & 2.8290 \\
\hline $\mathrm{H}$ & -3.06017 & -0.99109 & 1.37048 \\
\hline $\mathrm{H}$ & -2.39289 & 1.87657 & -1.3302 \\
\hline $\mathrm{H}$ & -1.69758 & 1.87030 & -2.9706 \\
\hline $\mathrm{H}$ & 1.60668 & 2.21426 & -2.41972 \\
\hline
\end{tabular}


Supporting Information for Donoghue et al.: Ligand and Substrate Effects on the Mechanisms of the Rhodium...

$\begin{array}{rrrr}\mathrm{H} & 1.98483 & 0.47370 & -2.43934 \\ \mathrm{H} & 3.23775 & 1.23298 & -0.76568 \\ \mathrm{H} & 4.35752 & 1.19500 & 0.58691 \\ \mathrm{H} & 3.36884 & -0.23151 & 0.23590 \\ \mathrm{C} & 3.36464 & 0.86191 & 0.25004\end{array}$

1b-MOLH-C

SCF Energy $\quad=-1316.986354$ hartree

Total Enthalpy $=-1316.650436$ hartree

$\begin{array}{rrrr}\mathrm{Rh} & 0.70613 & -0.28419 & 0.96488 \\ \mathrm{O} & 0.65696 & -0.29817 & 3.14454 \\ \mathrm{C} & 1.81032 & -0.27504 & 3.63230 \\ \mathrm{~N} & 2.90200 & -0.18160 & 2.87747 \\ \mathrm{C} & 2.80804 & 0.07560 & 1.44165 \\ \mathrm{C} & 2.14560 & 1.28785 & 1.08150 \\ \mathrm{H} & 1.84180 & 1.98637 & 1.85737 \\ \mathrm{H} & 2.38105 & 1.75094 & 0.12970 \\ \mathrm{H} & 3.81205 & -0.16471 & 3.32558 \\ \mathrm{H} & 1.95447 & -0.34253 & 4.71588 \\ \mathrm{P} & 0.62570 & -0.31710 & -1.31714 \\ \mathrm{P} & -1.59804 & 0.33028 & 0.79009 \\ \mathrm{C} & -1.07358 & 0.08572 & -1.88377 \\ \mathrm{C} & -2.02655 & 0.37301 & -0.99055 \\ \mathrm{H} & 0.06201 & -2.22859 & 1.14297 \\ \mathrm{H} & 0.83733 & -2.26541 & 1.10559 \\ \mathrm{C} & 1.65979 & 0.82792 & -2.32681 \\ \mathrm{C} & 0.96824 & -1.96175 & -2.07660 \\ \mathrm{C} & -2.04193 & 2.00771 & 1.41581 \\ \mathrm{C} & -2.90248 & -0.73919 & 1.53850 \\ \mathrm{H} & 2.72243 & 0.62321 & -2.17302 \\ \mathrm{H} & 0.79699 & -1.93458 & -3.15697 \\ \mathrm{H} & -3.09061 & 2.24507 & 1.21231 \\ \mathrm{H} & -2.74180 & -0.78480 & 2.61943 \\ \mathrm{H} & -3.04048 & 0.62059 & -1.30545 \\ \mathrm{H} & -1.27270 & 0.09128 & -2.95585 \\ \mathrm{H} & 1.45378 & 1.86448 & -2.05070 \\ \mathrm{H} & 1.43177 & 0.69329 & -3.38843 \\ \mathrm{H} & 2.00903 & -2.23925 & -1.88947 \\ \mathrm{H} & 0.31933 & -2.71796 & -1.62949 \\ \mathrm{H} & -2.83002 & -1.75234 & 1.13527 \\ \mathrm{H} & -3.90470 & -0.34490 & 1.34484 \\ \mathrm{H} & -1.87536 & 2.03255 & 2.49655 \\ \mathrm{H} & -1.40086 & 2.75809 & 0.94810 \\ \mathrm{H} & 3.89274 & -0.28501 & -0.38690 \\ \mathrm{H} & 4.92269 & -0.12310 & 1.03054 \\ \mathrm{H} & 3.99917 & -1.61417 & 0.78162 \\ \mathrm{C} & 3.96292 & -0.52630 & 0.67540\end{array}$

$1 b-M O L H-t s-A$

SCF Energy $=-1316.970255$ hartree

Total Enthalpy $=-1316.635740$ hartree Negative Frequencies: $-906.40 \mathrm{~cm}^{-1}$

$\mathrm{Rh}$

0.01546

$-0.01146$

$-0.01102$ 
Supporting Information for Donoghue et al.: Ligand and Substrate Effects on the Mechanisms of the Rhodium...

\begin{tabular}{|c|c|c|c|}
\hline O & 0.01090 & 0.00080 & 2.17123 \\
\hline C & 1.14944 & 0.01012 & 2.68119 \\
\hline $\mathrm{N}$ & 2.27375 & 0.03545 & 1.96076 \\
\hline C & 2.27837 & 0.27004 & 0.52554 \\
\hline $\mathrm{C}$ & 1.71676 & 1.47179 & 0.08990 \\
\hline $\mathrm{H}$ & 1.38840 & 2.22101 & 0.80134 \\
\hline $\mathrm{H}$ & 1.94280 & 1.83313 & -0.90609 \\
\hline $\mathrm{H}$ & 3.16211 & 0.02907 & 2.45084 \\
\hline $\mathrm{H}$ & 1.26546 & -0.02440 & 3.77033 \\
\hline $\mathrm{P}$ & -0.16703 & -0.18077 & -2.27684 \\
\hline $\mathrm{P}$ & -0.19469 & -2.43370 & -0.02409 \\
\hline $\mathrm{C}$ & -0.33719 & -1.94877 & -2.73084 \\
\hline $\mathrm{C}$ & -0.35555 & -2.89938 & -1.79001 \\
\hline $\mathrm{H}$ & -1.57731 & 0.22966 & -0.02470 \\
\hline $\mathrm{H}$ & -0.97736 & 1.26763 & -0.07453 \\
\hline C & -1.65319 & 0.60965 & -3.01962 \\
\hline $\mathrm{C}$ & 1.19630 & 0.42705 & -3.35456 \\
\hline C & -1.74477 & -3.06717 & 0.75111 \\
\hline C & 1.06750 & -3.62234 & 0.62008 \\
\hline $\mathrm{H}$ & -1.59189 & 1.69226 & -2.88105 \\
\hline $\mathrm{H}$ & 0.96688 & 0.20562 & -4.40144 \\
\hline $\mathrm{H}$ & -1.85965 & -4.14526 & 0.60487 \\
\hline $\mathrm{H}$ & 1.19813 & -3.45762 & 1.69340 \\
\hline $\mathrm{H}$ & -0.44788 & -2.19357 & -3.78779 \\
\hline $\mathrm{H}$ & -0.47652 & -3.94872 & -2.06041 \\
\hline $\mathrm{H}$ & 2.02711 & -3.46464 & 0.12284 \\
\hline $\mathrm{H}$ & 0.75092 & -4.65821 & 0.46300 \\
\hline $\mathrm{H}$ & -1.71119 & -2.85189 & 1.82299 \\
\hline $\mathrm{H}$ & -2.60402 & -2.54451 & 0.32580 \\
\hline $\mathrm{H}$ & -2.55173 & 0.24449 & -2.51830 \\
\hline $\mathrm{H}$ & -1.71548 & 0.38707 & -4.08898 \\
\hline $\mathrm{H}$ & 1.30027 & 1.50956 & -3.24343 \\
\hline $\mathrm{H}$ & 2.14055 & -0.05334 & -3.09272 \\
\hline C & 3.37518 & -0.47483 & -0.19450 \\
\hline $\mathrm{H}$ & 3.35541 & -0.25947 & -1.26364 \\
\hline $\mathrm{H}$ & 4.35946 & -0.15362 & 0.17671 \\
\hline $\mathrm{H}$ & 3.30746 & -1.55566 & -0.05180 \\
\hline
\end{tabular}

$1 b-M O L H-t s-C$

SCF Energy = $=-1316.973597$ hartree

Total Enthalpy $=-1316.639155$ hartree Negative Frequencies: $-930.50 \mathrm{~cm}^{-1}$

$\begin{array}{rrrr}\text { Rh } & 0.01470 & -0.01863 & -0.02151 \\ \mathrm{O} & -0.02752 & 0.02779 & 2.15670 \\ \mathrm{C} & 1.11262 & 0.07681 & 2.66291 \\ \mathrm{~N} & 2.22313 & 0.20791 & 1.93603 \\ \mathrm{C} & 2.19346 & 0.49550 & 0.51075 \\ \mathrm{C} & 1.54019 & 1.66891 & 0.12155 \\ \mathrm{H} & 1.16879 & 2.36112 & 0.86918 \\ \mathrm{H} & 1.74077 & 2.08659 & -0.85801 \\ \mathrm{H} & 3.12186 & 0.18838 & 2.40572 \\ \mathrm{H} & 1.23922 & -0.00122 & 3.74827 \\ \mathrm{P} & -0.12838 & -0.19675 & -2.29199 \\ \mathrm{P} & -1.92592 & 1.42138 & -0.23261 \\ \mathrm{C} & -1.57626 & 0.76527 & -2.87875\end{array}$


Supporting Information for Donoghue et al.: Ligand and Substrate Effects on the Mechanisms of the Rhodium...

$\begin{array}{rrrr}\mathrm{C} & -2.33850 & 1.44880 & -2.01795 \\ \mathrm{H} & -0.84658 & -1.38114 & 0.03124 \\ \mathrm{H} & 0.33304 & -1.61032 & 0.05587 \\ \mathrm{C} & 1.24479 & 0.40962 & -3.36034 \\ \mathrm{C} & -0.43155 & -1.89243 & -2.93684 \\ \mathrm{C} & -1.88580 & 3.20713 & 0.23762 \\ \mathrm{C} & -3.46619 & 0.82575 & 0.58605 \\ \mathrm{H} & 2.12872 & -0.21713 & -3.21889 \\ \mathrm{H} & -0.59353 & -1.87257 & -4.01855 \\ \mathrm{H} & -2.82761 & 3.70604 & -0.01097 \\ \mathrm{H} & -3.30389 & 0.81662 & 1.66770 \\ \mathrm{H} & -3.20161 & 2.01741 & -2.36493 \\ \mathrm{H} & -1.79490 & 0.75283 & -3.94709 \\ \mathrm{H} & 1.49519 & 1.44498 & -3.12053 \\ \mathrm{H} & 0.94609 & 0.35373 & -4.41149 \\ \mathrm{H} & 0.43375 & -2.52268 & -2.71460 \\ \mathrm{H} & -1.30842 & -2.31858 & -2.44481 \\ \mathrm{H} & -3.67994 & -0.19546 & 0.26329 \\ \mathrm{H} & -4.31967 & 1.47105 & 0.35736 \\ \mathrm{H} & -1.72377 & 3.28484 & 1.31681 \\ \mathrm{H} & -1.06459 & 3.71228 & -0.27606 \\ \mathrm{H} & 3.29645 & 0.11750 & -1.29850 \\ \mathrm{H} & 4.29183 & 0.21553 & 0.14802 \\ \mathrm{H} & 3.31916 & -1.23735 & -0.14794 \\ \mathrm{C} & 3.33061 & -0.14955 & -0.24195\end{array}$

\section{$1 \mathrm{~b}-\mathrm{DIHY}-\mathrm{A}$}

$\begin{array}{ll}\text { SCF Energy } & =-1316.980471 \text { hartree } \\ \text { Total Enthalpy } & =-1316.642880 \text { hartree }\end{array}$

$\begin{array}{rrrr}\text { Rh } & -0.14506 & 1.05528 & 0.97010 \\ \mathrm{O} & -0.31962 & 0.97554 & 3.16060 \\ \mathrm{C} & 0.69923 & 0.86932 & 3.85739 \\ \mathrm{~N} & 1.95967 & 0.86286 & 3.38117 \\ \mathrm{C} & 2.32174 & 1.10994 & 2.01092 \\ \mathrm{C} & 1.95849 & 2.28999 & 1.43885 \\ \mathrm{H} & 1.50030 & 3.08127 & 2.01777 \\ \mathrm{H} & 2.32486 & 2.54541 & 0.45229 \\ \mathrm{H} & 2.70706 & 0.67400 & 4.03956 \\ \mathrm{H} & 0.61453 & 0.74574 & 4.94403 \\ \mathrm{P} & -0.24262 & 1.15909 & -1.28424 \\ \mathrm{P} & -0.31156 & -1.39206 & 0.69137 \\ \mathrm{C} & -0.51476 & -0.53161 & -1.93608 \\ \mathrm{C} & -0.55026 & -1.59040 & -1.11859 \\ \mathrm{H} & -1.67463 & 1.02668 & 0.81448 \\ \mathrm{H} & -0.50692 & 2.58919 & 0.94267 \\ \mathrm{C} & -1.59799 & 2.15263 & -2.02425 \\ \mathrm{C} & 1.25686 & 1.74888 & -2.17717 \\ \mathrm{C} & -1.86697 & -2.07912 & 1.41240 \\ \mathrm{C} & 0.90294 & -2.73972 & 1.07143 \\ \mathrm{H} & -1.45526 & 3.20295 & -1.75849 \\ \mathrm{H} & 1.11848 & 1.65142 & -3.25843 \\ \mathrm{H} & -2.05052 & -3.10317 & 1.07440 \\ \mathrm{H} & 1.09143 & -2.76756 & 2.14885 \\ \mathrm{H} & -0.66039 & -0.64536 & -3.01094 \\ \mathrm{H} & -0.72081 & -2.59024 & -1.51944\end{array}$


Supporting Information for Donoghue et al.: Ligand and Substrate Effects on the Mechanisms of the Rhodium...

$\begin{array}{rrrr}\mathrm{H} & 1.84792 & -2.56115 & 0.55331 \\ \mathrm{H} & 0.51283 & -3.71461 & 0.76361 \\ \mathrm{H} & -1.78322 & -2.07219 & 2.50307 \\ \mathrm{H} & -2.70987 & -1.44388 & 1.13228 \\ \mathrm{H} & -2.55625 & 1.82172 & -1.61914 \\ \mathrm{H} & -1.60455 & 2.05157 & -3.11359 \\ \mathrm{H} & 1.43086 & 2.80205 & -1.94031 \\ \mathrm{H} & 2.13016 & 1.16439 & -1.87889 \\ \mathrm{H} & 3.50829 & 0.34503 & 0.38520 \\ \mathrm{H} & 4.29702 & 0.28035 & 1.96077 \\ \mathrm{H} & 3.03491 & -0.89118 & 1.57475 \\ \mathrm{C} & 3.33500 & 0.15020 & 1.44542\end{array}$

1b-DIHY-C

SCF Energy = $\quad-1316.984823$ hartree

Total Enthalpy $=-1316.647475$ hartree

$\begin{array}{rrrr}\mathrm{Rh} & 0.52930 & -0.66261 & 0.94366 \\ \mathrm{O} & 0.52976 & -0.73960 & 3.14288 \\ \mathrm{C} & 1.62790 & -0.65352 & 3.70992 \\ \mathrm{~N} & 2.77488 & -0.30256 & 3.09788 \\ \mathrm{C} & 2.87199 & 0.14129 & 1.73317 \\ \mathrm{C} & 2.13211 & 1.21204 & 1.33033 \\ \mathrm{H} & 1.54230 & 1.78244 & 2.03616 \\ \mathrm{H} & 2.29302 & 1.63983 & 0.34797 \\ \mathrm{H} & 3.63686 & -0.39445 & 3.62319 \\ \mathrm{H} & 1.72120 & -0.88168 & 4.77865 \\ \mathrm{P} & 0.40747 & -0.86217 & -1.30175 \\ \mathrm{P} & -1.28182 & 0.97583 & 0.72374 \\ \mathrm{C} & -0.94968 & 0.20893 & -1.91501 \\ \mathrm{C} & -1.65813 & 0.97239 & -1.07394 \\ \mathrm{H} & -0.58138 & -1.72726 & 0.88467 \\ \mathrm{H} & 1.41848 & -1.97481 & 0.91113 \\ \mathrm{C} & 1.87133 & -0.34504 & -2.29335 \\ \mathrm{C} & 0.01056 & -2.52611 & -1.97009 \\ \mathrm{C} & -1.16979 & 2.77856 & 1.12176 \\ \mathrm{C} & -2.87766 & 0.49497 & 1.51378 \\ \mathrm{H} & 2.70844 & -1.01354 & -2.07595 \\ \mathrm{H} & -0.11184 & -2.49035 & -3.05664 \\ \mathrm{H} & -2.07695 & 3.31006 & 0.81865 \\ \mathrm{H} & -2.75812 & 0.54082 & 2.60012 \\ \mathrm{H} & -2.46790 & 1.60049 & -1.44685 \\ \mathrm{H} & -1.16812 & 0.19514 & -2.98340 \\ \mathrm{H} & 2.15718 & 0.67990 & -2.04682 \\ \mathrm{H} & 1.64587 & -0.40109 & -3.36273 \\ \mathrm{H} & 0.82261 & -3.21280 & -1.71850 \\ \mathrm{H} & -0.90810 & -2.89455 & -1.50929 \\ \mathrm{H} & -3.12431 & -0.53324 & 1.24070 \\ \mathrm{H} & -3.69308 & 1.16017 & 1.21513 \\ \mathrm{H} & -1.04258 & 2.89894 & 2.20190 \\ \mathrm{H} & -0.31066 & 3.22663 & 0.61650 \\ \mathrm{H} & 4.03282 & -0.10240 & -0.06159 \\ \mathrm{H} & 4.97373 & -0.11496 & 1.42933 \\ \mathrm{H} & 4.01363 & -1.53841 & 0.99110 \\ \mathrm{C} & 4.03073 & -0.44706 & 0.97361\end{array}$


Supporting Information for Donoghue et al.: Ligand and Substrate Effects on the Mechanisms of the Rhodium...

$1 b-D I H Y-t s-A$

SCF Energy $=-1316.967116$ hartree
Total Enthalpy $=-1316.631683$ hartree
Negative Frequencies: $-771.79 \mathrm{~cm}^{-1}$

$\begin{array}{rrrr}\mathrm{Rh} & 0.02688 & -0.02072 & -0.01596 \\ \mathrm{O} & -0.02099 & -0.00695 & 2.18809 \\ \mathrm{C} & 1.10886 & -0.00483 & 2.71925 \\ \mathrm{~N} & 2.24427 & 0.07168 & 2.01737 \\ \mathrm{C} & 2.25059 & 0.31313 & 0.58855 \\ \mathrm{C} & 1.71658 & 1.56741 & 0.18433 \\ \mathrm{H} & 1.54234 & 2.32619 & 0.94369 \\ \mathrm{H} & 2.02149 & 1.95941 & -0.78094 \\ \mathrm{H} & 3.12696 & 0.04039 & 2.51708 \\ \mathrm{H} & 1.21033 & -0.08157 & 3.80767 \\ \mathrm{P} & -0.29529 & -0.13424 & -2.25759 \\ \mathrm{P} & -0.33496 & -2.35339 & -0.00380 \\ \mathrm{C} & -0.73164 & -1.86330 & -2.67970 \\ \mathrm{C} & -0.75237 & -2.80471 & -1.72957 \\ \mathrm{H} & -1.54363 & 0.12982 & -0.09691 \\ \mathrm{H} & 0.14937 & 1.61295 & -0.10081 \\ \mathrm{C} & -1.65208 & 0.87699 & -2.97211 \\ \mathrm{C} & 1.13381 & 0.24742 & -3.35763 \\ \mathrm{C} & -1.78832 & -2.87222 & 0.99694 \\ \mathrm{C} & 0.96526 & -3.57323 & 0.47310 \\ \mathrm{H} & -1.43193 & 1.93512 & -2.80700 \\ \mathrm{H} & 0.86461 & 0.07398 & -4.40399 \\ \mathrm{H} & -1.98782 & -3.94254 & 0.89198 \\ \mathrm{H} & 1.23054 & -3.41847 & 1.52285 \\ \mathrm{H} & -0.97697 & -2.09449 & -3.71641 \\ \mathrm{H} & -1.01385 & -3.83623 & -1.96558 \\ \mathrm{H} & 1.85843 & -3.42905 & -0.13925 \\ \mathrm{H} & 0.60868 & -4.60052 & 0.35026 \\ \mathrm{H} & -1.58678 & -2.64165 & 2.04660 \\ \mathrm{H} & -2.66380 & -2.29932 & 0.68487 \\ \mathrm{H} & -2.58829 & 0.63820 & -2.46433 \\ \mathrm{H} & -1.75303 & 0.69335 & -4.04595 \\ \mathrm{H} & 1.41647 & 1.29777 & -3.24313 \\ \mathrm{H} & 1.98765 & -0.38299 & -3.10155 \\ \mathrm{H} & 3.36231 & -0.12827 & -1.20408 \\ \mathrm{H} & 4.35430 & -0.05769 & 0.24844 \\ \mathrm{H} & 3.32884 & -1.46743 & -0.04299 \\ \mathrm{C} & 3.37689 & -0.37987 & -0.14193\end{array}$

$1 b-D I H Y-t s-C$

SCF Energy = $=-1316.964222$ hartree Total Enthalpy $=-1316.629364$ hartree Negative Frequencies: $-727.41 \mathrm{~cm}^{-1}$

$\begin{array}{rrrr}\text { Rh } & 0.02002 & -0.01332 & -0.01377 \\ \mathrm{O} & -0.04206 & 0.00305 & 2.20254 \\ \mathrm{C} & 1.07734 & 0.02916 & 2.74224 \\ \mathrm{~N} & 2.22345 & 0.17985 & 2.06362 \\ \mathrm{C} & 2.24845 & 0.47469 & 0.63398 \\ \mathrm{C} & 1.53942 & 1.65283 & 0.24448\end{array}$


Supporting Information for Donoghue et al.: Ligand and Substrate Effects on the Mechanisms of the Rhodium...

$\begin{array}{rrrr}\mathrm{H} & 1.17265 & 2.31581 & 1.01947 \\ \mathrm{H} & 1.81694 & 2.12397 & -0.69198 \\ \mathrm{H} & 3.10346 & 0.13619 & 2.56234 \\ \mathrm{H} & 1.17544 & -0.08958 & 3.82768 \\ \mathrm{P} & -0.08445 & -0.38261 & -2.24156 \\ \mathrm{P} & -2.01041 & 1.09906 & -0.26730 \\ \mathrm{C} & -1.69035 & 0.25295 & -2.85316 \\ \mathrm{C} & -2.51677 & 0.88640 & -2.01399 \\ \mathrm{H} & -0.75721 & -1.40478 & -0.01264 \\ \mathrm{H} & 1.52056 & -0.75533 & 0.16695 \\ \mathrm{C} & 1.15896 & 0.46395 & -3.30666 \\ \mathrm{C} & -0.01846 & -2.12490 & -2.81806 \\ \mathrm{C} & -2.14915 & 2.91193 & 0.03233 \\ \mathrm{C} & -3.37581 & 0.38337 & 0.73242 \\ \mathrm{H} & 2.15389 & 0.05664 & -3.10462 \\ \mathrm{H} & -0.12866 & -2.18113 & -3.90530 \\ \mathrm{H} & -3.15736 & 3.27321 & -0.19308 \\ \mathrm{H} & -3.13296 & 0.50703 & 1.79142 \\ \mathrm{H} & -3.47589 & 1.27997 & -2.34998 \\ \mathrm{H} & -1.94347 & 0.10284 & -3.90274 \\ \mathrm{H} & 1.16301 & 1.53656 & -3.10041 \\ \mathrm{H} & 0.92720 & 0.30244 & -4.36443 \\ \mathrm{H} & 0.94268 & -2.55940 & -2.53071 \\ \mathrm{H} & -0.81201 & -2.69609 & -2.33303 \\ \mathrm{H} & -3.45608 & -0.68462 & 0.52072 \\ \mathrm{H} & -4.32765 & 0.87878 & 0.51945 \\ \mathrm{H} & -1.92949 & 3.11386 & 1.08454 \\ \mathrm{H} & -1.42422 & 3.44698 & -0.58482 \\ \mathrm{C} & 3.57307 & 0.09572 & -0.01088 \\ \mathrm{H} & 3.53400 & 0.28131 & -1.08546 \\ \mathrm{H} & 4.36846 & 0.72820 & 0.40107 \\ \mathrm{H} & 3.83019 & -0.95346 & 0.15808\end{array}$

1b-ALHY -A

SCF Energy $\quad=-1317.006446$ hartree Total Enthalpy $=-1316.666405$ hartree

$\begin{array}{rrrr}\mathrm{Rh} & 0.78877 & -0.13858 & 0.93238 \\ \mathrm{O} & 0.83337 & -0.27819 & 3.05478 \\ \mathrm{C} & 2.02276 & -0.30759 & 3.47131 \\ \mathrm{~N} & 3.05441 & -0.32872 & 2.64813 \\ \mathrm{C} & 2.86126 & -0.48600 & 1.17872 \\ \mathrm{C} & 3.92421 & 0.34861 & 0.47479 \\ \mathrm{H} & 4.93824 & 0.05430 & 0.78889 \\ \mathrm{H} & 3.88160 & 0.19736 & -0.60723 \\ \mathrm{H} & 3.99226 & -0.36718 & 3.03931 \\ \mathrm{H} & 2.22206 & -0.31335 & 4.54770 \\ \mathrm{P} & 0.67918 & 0.17350 & -1.33854 \\ \mathrm{P} & -1.63455 & 0.08140 & 0.85602 \\ \mathrm{C} & -1.08064 & 0.37671 & -1.82068 \\ \mathrm{C} & -2.05767 & 0.35135 & -0.90618 \\ \mathrm{H} & 1.04033 & 1.35154 & 0.96923 \\ \mathrm{H} & 3.80495 & 1.41562 & 0.67921 \\ \mathrm{C} & 1.47496 & 1.67412 & -2.04757 \\ \mathrm{C} & 1.26854 & -1.18992 & -2.43137 \\ \mathrm{C} & -2.38270 & 1.51178 & 1.74405\end{array}$


Supporting Information for Donoghue et al.: Ligand and Substrate Effects on the Mechanisms of the Rhodium...

$\begin{array}{rrrr}\mathrm{C} & -2.70300 & -1.33695 & 1.36160 \\ \mathrm{H} & 2.55563 & 1.62763 & -1.89701 \\ \mathrm{H} & 0.99594 & -0.98770 & -3.47175 \\ \mathrm{H} & -3.45723 & 1.58507 & 1.55317 \\ \mathrm{H} & -2.55834 & -1.52338 & 2.42964 \\ \mathrm{H} & -3.09889 & 0.48613 & -1.20031 \\ \mathrm{H} & -1.30033 & 0.53341 & -2.87744 \\ \mathrm{H} & 1.09084 & 2.56337 & -1.54294 \\ \mathrm{H} & 1.26701 & 1.74909 & -3.11898 \\ \mathrm{H} & 2.35641 & -1.27435 & -2.36631 \\ \mathrm{H} & 0.82212 & -2.13909 & -2.12500 \\ \mathrm{H} & -2.41721 & -2.23657 & 0.81067 \\ \mathrm{H} & -3.76087 & -1.12535 & 1.17878 \\ \mathrm{H} & -2.21810 & 1.37960 & 2.81707 \\ \mathrm{H} & -1.89359 & 2.43708 & 1.43168 \\ \mathrm{C} & 2.99189 & -1.98517 & 0.85125 \\ \mathrm{H} & 2.97650 & -2.15647 & -0.22737 \\ \mathrm{H} & 3.93896 & -2.39507 & 1.23186 \\ \mathrm{H} & 2.18947 & -2.58489 & 1.30396\end{array}$

\section{1b-ALHY-C}

$\begin{array}{lll}\text { SCF Energy } & =-1317.006100 & \text { hartree } \\ \text { Total Enthalpy } & =-1316.665111 \text { hartree }\end{array}$

$\begin{array}{rrrr}\mathrm{Rh} & 0.63857 & 0.16492 & 0.80598 \\ \mathrm{O} & 0.64290 & 0.15385 & 2.94652 \\ \mathrm{C} & 1.65230 & 0.19689 & 3.68450 \\ \mathrm{~N} & 2.92184 & 0.07898 & 3.31711 \\ \mathrm{C} & 3.45711 & -0.20018 & 1.95984 \\ \mathrm{C} & 2.70017 & 0.56055 & 0.86950 \\ \mathrm{H} & 2.77761 & 1.64581 & 1.05108 \\ \mathrm{H} & 3.21237 & 0.35320 & -0.07462 \\ \mathrm{H} & 3.59883 & 0.10391 & 4.06987 \\ \mathrm{H} & 1.50008 & 0.35088 & 4.76047 \\ \mathrm{P} & -1.75955 & -0.32663 & 0.68792 \\ \mathrm{P} & 0.53033 & 0.30983 & -1.45525 \\ \mathrm{C} & -2.14639 & -0.31268 & -1.10705 \\ \mathrm{C} & -1.18507 & -0.04143 & -1.99928 \\ \mathrm{H} & 0.98014 & -1.28317 & 0.55242 \\ \mathrm{H} & 3.35104 & -1.27974 & 1.78710 \\ \mathrm{C} & -2.34726 & -1.98011 & 1.25792 \\ \mathrm{C} & -3.01040 & 0.82540 & 1.41043 \\ \mathrm{C} & 1.56251 & -0.82896 & -2.46379 \\ \mathrm{C} & 0.90251 & 1.97874 & -2.13838 \\ \mathrm{H} & -2.22175 & -2.04091 & 2.34267 \\ \mathrm{H} & -4.03039 & 0.52744 & 1.14970 \\ \mathrm{H} & 1.37406 & -0.68027 & -3.53103 \\ \mathrm{H} & 1.94922 & 2.22441 & -1.93977 \\ \mathrm{H} & -3.16388 & -0.52188 & -1.43907 \\ \mathrm{H} & -1.39672 & -0.02506 & -3.06894 \\ \mathrm{H} & 0.26921 & 2.72785 & -1.65700 \\ \mathrm{H} & 0.72895 & 2.00537 & -3.21833 \\ \mathrm{H} & 2.61960 & -0.64024 & -2.26053 \\ \mathrm{H} & 1.33524 & -1.86341 & -2.19735 \\ \mathrm{H} & -1.74491 & -2.76610 & 0.79658 \\ \mathrm{H} & -3.40141 & -2.13950 & 1.01270\end{array}$


Supporting Information for Donoghue et al.: Ligand and Substrate Effects on the Mechanisms of the Rhodium...

$\begin{array}{rrrr}\mathrm{H} & -2.90642 & 0.81762 & 2.49923 \\ \mathrm{H} & -2.83242 & 1.84304 & 1.05388 \\ \mathrm{H} & 5.41138 & -0.12560 & 1.03190 \\ \mathrm{H} & 5.10834 & 1.21301 & 2.15282 \\ \mathrm{H} & 5.48246 & -0.41559 & 2.77074 \\ \mathrm{C} & 4.95388 & 0.14149 & 1.98745\end{array}$

\section{ZDMP Ligand, Substrate c}

\begin{tabular}{|c|c|c|c|}
\hline \multicolumn{2}{|c|}{ SCF Energy } & \multicolumn{2}{|c|}{-1408.040756 hartree } \\
\hline Total & Enthalpy $=$ & 20088 hart & \\
\hline $\mathrm{Rh}$ & 0.17223 & 1.02249 & 0.93666 \\
\hline $\mathrm{O}$ & 0.55523 & 0.74358 & 3.06191 \\
\hline $\mathrm{C}$ & 1.12128 & 1.72386 & 3.58236 \\
\hline $\mathrm{N}$ & 1.49701 & 2.79349 & 2.87599 \\
\hline $\mathrm{C}$ & 1.21734 & 2.92716 & 1.45631 \\
\hline $\mathrm{C}$ & -0.10204 & 3.22602 & 1.06830 \\
\hline $\mathrm{H}$ & -0.26925 & 3.66669 & 0.09099 \\
\hline $\mathrm{H}$ & 1.94843 & 3.56615 & 3.35465 \\
\hline $\mathrm{H}$ & 1.34160 & 1.74259 & 4.65535 \\
\hline $\mathrm{P}$ & -0.24173 & 0.96388 & -1.30825 \\
\hline $\mathrm{P}$ & -0.57362 & -1.20022 & 0.88954 \\
\hline $\mathrm{C}$ & -0.85543 & -0.70646 & -1.76923 \\
\hline $\mathrm{C}$ & -1.00394 & -1.64559 & -0.82861 \\
\hline $\mathrm{C}$ & -1.54592 & 2.07199 & -2.00109 \\
\hline $\mathrm{C}$ & 1.17861 & 1.21405 & -2.45902 \\
\hline $\mathrm{C}$ & -2.07173 & -1.53874 & 1.89994 \\
\hline $\mathrm{C}$ & 0.64720 & -2.45627 & 1.45386 \\
\hline $\mathrm{H}$ & -1.21788 & 3.11468 & -1.97060 \\
\hline $\mathrm{H}$ & 0.87746 & 1.01751 & -3.49270 \\
\hline $\mathrm{H}$ & -2.37103 & -2.58810 & 1.81744 \\
\hline $\mathrm{H}$ & 0.92414 & -2.22875 & 2.48688 \\
\hline $\mathrm{H}$ & -1.09553 & -0.90138 & -2.81476 \\
\hline $\mathrm{H}$ & -1.37096 & -2.64370 & -1.06588 \\
\hline $\mathrm{H}$ & 1.54404 & -2.41309 & 0.83203 \\
\hline $\mathrm{H}$ & 0.22108 & -3.46308 & 1.40984 \\
\hline $\mathrm{H}$ & -1.84439 & -1.30891 & 2.94435 \\
\hline $\mathrm{H}$ & -2.89251 & -0.89647 & 1.57401 \\
\hline $\mathrm{H}$ & -2.46162 & 1.97596 & -1.41314 \\
\hline $\mathrm{H}$ & -1.75683 & 1.81042 & -3.04242 \\
\hline $\mathrm{H}$ & 1.53408 & 2.24565 & -2.39090 \\
\hline $\mathrm{H}$ & 1.99699 & 0.54363 & -2.18770 \\
\hline $\mathrm{H}$ & 2.15581 & 3.47232 & -0.40664 \\
\hline $\mathrm{H}$ & 2.89112 & 4.20627 & 1.02172 \\
\hline $\mathrm{H}$ & 3.17930 & 2.49575 & 0.67080 \\
\hline $\mathrm{C}$ & 2.42649 & 3.28841 & 0.63429 \\
\hline $\mathrm{N}$ & -2.04614 & 3.51772 & 2.76158 \\
\hline C & -1.17194 & 3.38800 & 2.00367 \\
\hline
\end{tabular}

$1 c-M O L H-A$

SCF Energy = $\quad-1409.215439$ hartree 
Supporting Information for Donoghue et al.: Ligand and Substrate Effects on the Mechanisms of the Rhodium...

Total Enthalpy $=-1408.878914$ hartree

\begin{tabular}{|c|c|c|c|}
\hline $\mathrm{Rh}$ & 0.15615 & 1.00543 & 0.95405 \\
\hline 0 & 0.32364 & 0.87382 & 3.10810 \\
\hline C & 1.51679 & 0.92970 & 3.47424 \\
\hline $\mathrm{N}$ & 2.51607 & 1.01818 & 2.59566 \\
\hline C & 2.25663 & 1.32619 & 1.19229 \\
\hline C & 1.62965 & 2.60858 & 0.93573 \\
\hline $\mathrm{H}$ & 1.77930 & 3.04181 & -0.04898 \\
\hline $\mathrm{H}$ & 3.46949 & 1.08911 & 2.93781 \\
\hline $\mathrm{H}$ & 1.77798 & 0.88820 & 4.53620 \\
\hline $\mathrm{P}$ & -0.15930 & 0.92448 & -1.32509 \\
\hline P & -0.64283 & -1.24764 & 0.91400 \\
\hline C & -0.77352 & -0.74240 & -1.78117 \\
\hline C & -0.97853 & -1.66808 & -0.83739 \\
\hline $\mathrm{H}$ & -1.79508 & 1.54500 & 1.25062 \\
\hline $\mathrm{H}$ & -1.41355 & 2.21735 & 1.18974 \\
\hline C & -1.48520 & 2.06872 & -1.90206 \\
\hline C & 1.18707 & 1.22563 & -2.54544 \\
\hline C & -2.21015 & -1.66972 & 1.78932 \\
\hline C & 0.51376 & -2.56123 & 1.49550 \\
\hline $\mathrm{H}$ & -1.16543 & 3.10279 & -1.74469 \\
\hline $\mathrm{H}$ & 0.77917 & 1.16783 & -3.55906 \\
\hline $\mathrm{H}$ & -2.47622 & -2.72190 & 1.64996 \\
\hline $\mathrm{H}$ & 0.69856 & -2.41864 & 2.56399 \\
\hline $\mathrm{H}$ & -0.97322 & -0.94681 & -2.83336 \\
\hline $\mathrm{H}$ & -1.35632 & -2.65819 & -1.09299 \\
\hline $\mathrm{H}$ & 1.46329 & -2.48528 & 0.96144 \\
\hline $\mathrm{H}$ & 0.09029 & -3.55858 & 1.34295 \\
\hline $\mathrm{H}$ & -2.07952 & -1.47297 & 2.85731 \\
\hline $\mathrm{H}$ & -3.02423 & -1.04096 & 1.42015 \\
\hline $\mathrm{H}$ & -2.40062 & 1.89653 & -1.33233 \\
\hline $\mathrm{H}$ & -1.69018 & 1.91746 & -2.96604 \\
\hline $\mathrm{H}$ & 1.61466 & 2.22185 & -2.40391 \\
\hline $\mathrm{H}$ & 1.97389 & 0.47661 & -2.44146 \\
\hline $\mathrm{H}$ & 3.20932 & 1.10533 & -0.73028 \\
\hline $\mathrm{H}$ & 4.31909 & 1.10134 & 0.63164 \\
\hline $\mathrm{H}$ & 3.33182 & -0.33340 & 0.30895 \\
\hline C & 3.32911 & 0.75993 & 0.29546 \\
\hline $\mathrm{N}$ & 1.26894 & 4.34223 & 2.83215 \\
\hline C & 1.43192 & 3.57224 & 1.97359 \\
\hline
\end{tabular}

1c-MOLH-C

SCF Energy = $\quad-1409.219822$ hartree

Total Enthalpy $=-1408.883118$ hartree

$\begin{array}{rrrr}\text { Rh } & 0.71793 & -0.31400 & 0.96039 \\ \mathrm{O} & 0.62957 & -0.37330 & 3.13236 \\ \mathrm{C} & 1.76552 & -0.29647 & 3.65073 \\ \mathrm{~N} & 2.87164 & -0.17346 & 2.91682 \\ \mathrm{C} & 2.79887 & 0.05780 & 1.48317 \\ \mathrm{C} & 2.13567 & 1.27761 & 1.07087 \\ \mathrm{H} & 2.40806 & 1.67945 & 0.10007 \\ \mathrm{H} & 3.76916 & -0.10208 & 3.38525 \\ \mathrm{H} & 1.88519 & -0.34175 & 4.73746 \\ \mathrm{P} & 0.61998 & -0.31777 & -1.33092\end{array}$


Supporting Information for Donoghue et al.: Ligand and Substrate Effects on the Mechanisms of the Rhodium...

$\begin{array}{rrrr}\mathrm{P} & -1.58332 & 0.33772 & 0.80805 \\ \mathrm{C} & -1.07966 & 0.10945 & -1.87282 \\ \mathrm{C} & -2.02227 & 0.39721 & -0.96859 \\ \mathrm{H} & 0.06093 & -2.18854 & 1.08488 \\ \mathrm{H} & 0.84198 & -2.25461 & 1.07153 \\ \mathrm{C} & 1.66294 & 0.83261 & -2.32371 \\ \mathrm{C} & 0.94867 & -1.95632 & -2.10523 \\ \mathrm{C} & -1.98521 & 2.01007 & 1.46325 \\ \mathrm{C} & -2.87146 & -0.73835 & 1.56931 \\ \mathrm{H} & 2.72423 & 0.61748 & -2.17425 \\ \mathrm{H} & 0.77507 & -1.91508 & -3.18490 \\ \mathrm{H} & -3.03701 & 2.25791 & 1.29169 \\ \mathrm{H} & -2.68328 & -0.79999 & 2.64507 \\ \mathrm{H} & -3.03497 & 0.66063 & -1.27398 \\ \mathrm{H} & -1.28656 & 0.13039 & -2.94319 \\ \mathrm{H} & 1.46307 & 1.86832 & -2.03948 \\ \mathrm{H} & 1.43416 & 0.71096 & -3.38671 \\ \mathrm{H} & 1.98816 & -2.24326 & -1.92387 \\ \mathrm{H} & 0.29437 & -2.71237 & -1.66586 \\ \mathrm{H} & -2.81682 & -1.74541 & 1.14840 \\ \mathrm{H} & -3.87473 & -0.33271 & 1.40750 \\ \mathrm{H} & -1.78166 & 2.02264 & 2.53740 \\ \mathrm{H} & -1.34756 & 2.76014 & 0.99143 \\ \mathrm{H} & 3.93372 & -0.26901 & -0.32313 \\ \mathrm{H} & 4.92807 & -0.05808 & 1.11530 \\ \mathrm{H} & 4.06322 & -1.58374 & 0.85868 \\ \mathrm{C} & 3.99192 & -0.49899 & 0.74255 \\ \mathrm{~N} & 1.36358 & 3.08321 & 2.77044 \\ \mathrm{C} & 1.72130 & 2.28070 & 2.00638\end{array}$

1c-MOLH-ts $-\mathrm{A}$

SCF Energy = $\quad-1409.198396$ hartree Total Enthalpy $=-1408.863170$ hartree Negative Frequencies: $-824.62 \mathrm{~cm}^{-1}$

$\begin{array}{rrrr}\text { Rh } & 0.00330 & 0.00266 & 0.00753 \\ \mathrm{O} & 0.01881 & 0.00333 & 2.18164 \\ \mathrm{C} & 1.16413 & -0.00552 & 2.67384 \\ \mathrm{~N} & 2.27227 & -0.04538 & 1.92429 \\ \mathrm{C} & 2.23529 & 0.15390 & 0.48967 \\ \mathrm{C} & 1.74216 & 1.40732 & 0.03481 \\ \mathrm{H} & 1.98211 & 1.69864 & -0.98201 \\ \mathrm{H} & 3.17376 & -0.04069 & 2.39089 \\ \mathrm{H} & 1.30095 & -0.00672 & 3.76001 \\ \mathrm{P} & -0.24583 & -0.11806 & -2.26339 \\ \mathrm{P} & -0.26650 & -2.42307 & -0.03438 \\ \mathrm{C} & -0.43792 & -1.87773 & -2.73442 \\ \mathrm{C} & -0.45518 & -2.84328 & -1.80851 \\ \mathrm{H} & -1.58983 & 0.15022 & 0.02910 \\ \mathrm{H} & -0.97357 & 1.27436 & -0.03685 \\ \mathrm{C} & -1.75058 & 0.69841 & -2.93294 \\ \mathrm{C} & 1.08735 & 0.51291 & -3.36372 \\ \mathrm{C} & -1.82073 & -3.03017 & 0.74937 \\ \mathrm{C} & 0.98068 & -3.64326 & 0.57457 \\ \mathrm{H} & -1.68000 & 1.77573 & -2.76088 \\ \mathrm{H} & 0.83202 & 0.30386 & -4.40701\end{array}$


Supporting Information for Donoghue et al.: Ligand and Substrate Effects on the Mechanisms of the Rhodium...

$\begin{array}{rrrr}\mathrm{H} & -1.96280 & -4.10175 & 0.58132 \\ \mathrm{H} & 1.13713 & -3.49188 & 1.64630 \\ \mathrm{H} & -0.56770 & -2.10626 & -3.79276 \\ \mathrm{H} & -0.59430 & -3.88560 & -2.09650 \\ \mathrm{H} & 1.93311 & -3.50817 & 0.05805 \\ \mathrm{H} & 0.63258 & -4.66839 & 0.41460 \\ \mathrm{H} & -1.76626 & -2.83963 & 1.82505 \\ \mathrm{H} & -2.67315 & -2.47861 & 0.34759 \\ \mathrm{H} & -2.63457 & 0.32071 & -2.41535 \\ \mathrm{H} & -1.84652 & 0.51034 & -4.00626 \\ \mathrm{H} & 1.18304 & 1.59555 & -3.24323 \\ \mathrm{H} & 2.04330 & 0.03829 & -3.13556 \\ \mathrm{H} & 3.24646 & -0.43168 & -1.32414 \\ \mathrm{H} & 4.30176 & -0.27630 & 0.07558 \\ \mathrm{H} & 3.24201 & -1.68448 & -0.06752 \\ \mathrm{C} & 3.30567 & -0.60990 & -0.24962 \\ \mathrm{~N} & 1.35041 & 3.38561 & 1.66661 \\ \mathrm{C} & 1.50624 & 2.50106 & 0.92704\end{array}$

1c-MOLH-ts-C

SCF Energy = $\quad-1409.206641$ hartree Total Enthalpy $=-1408.871542$ hartree Negative Frequencies: $-851.64 \mathrm{~cm}^{-1}$

$\begin{array}{rrrr}\text { Rh } & 0.00157 & -0.00665 & -0.00475 \\ \mathrm{O} & 0.00268 & -0.01508 & 2.16511 \\ \mathrm{C} & 1.15608 & -0.00731 & 2.64150 \\ \mathrm{~N} & 2.24568 & 0.05954 & 1.87178 \\ \mathrm{C} & 2.16178 & 0.34076 & 0.45201 \\ \mathrm{C} & 1.59362 & 1.59604 & 0.08104 \\ \mathrm{H} & 1.81029 & 1.96868 & -0.91419 \\ \mathrm{H} & 3.16126 & 0.03422 & 2.30842 \\ \mathrm{H} & 1.31184 & -0.07239 & 3.72267 \\ \mathrm{P} & -0.23466 & -0.13270 & -2.28151 \\ \mathrm{P} & -2.02923 & 1.34304 & -0.09683 \\ \mathrm{C} & -1.83706 & 0.62603 & -2.74644 \\ \mathrm{C} & -2.59569 & 1.24714 & -1.83635 \\ \mathrm{H} & -0.91603 & -1.32192 & 0.03793 \\ \mathrm{H} & 0.32257 & -1.58401 & 0.01942 \\ \mathrm{C} & 0.97158 & 0.73029 & -3.37404 \\ \mathrm{C} & -0.30454 & -1.83103 & -2.97738 \\ \mathrm{C} & -2.06452 & 3.14351 & 0.29192 \\ \mathrm{C} & -3.41550 & 0.66254 & 0.90403 \\ \mathrm{H} & 1.96259 & 0.28032 & -3.27647 \\ \mathrm{H} & -0.52481 & -1.80086 & -4.04854 \\ \mathrm{H} & -3.08137 & 3.53672 & 0.19733 \\ \mathrm{H} & -3.13379 & 0.71104 & 1.95975 \\ \mathrm{H} & -3.54102 & 1.70974 & -2.12069 \\ \mathrm{H} & -2.14037 & 0.56074 & -3.79184 \\ \mathrm{H} & 1.02900 & 1.79067 & -3.11928 \\ \mathrm{H} & 0.65264 & 0.63797 & -4.41682 \\ \mathrm{H} & 0.65887 & -2.32427 & -2.82350 \\ \mathrm{H} & -1.07663 & -2.40613 & -2.46221 \\ \mathrm{H} & -3.58465 & -0.38340 & 0.63969 \\ \mathrm{H} & -4.33460 & 1.23603 & 0.75208 \\ \mathrm{H} & -1.70736 & 3.29920 & 1.31319 \\ & & & \end{array}$


Supporting Information for Donoghue et al.: Ligand and Substrate Effects on the Mechanisms of the Rhodium...

$\begin{array}{rrrr}\mathrm{H} & -1.39969 & 3.68768 & -0.38236 \\ \mathrm{H} & 3.17722 & -0.06773 & -1.40474 \\ \mathrm{H} & 4.23820 & 0.03154 & -0.00478 \\ \mathrm{H} & 3.22793 & -1.40935 & -0.24119 \\ \mathrm{C} & 3.25544 & -0.32302 & -0.34731 \\ \mathrm{~N} & 1.01274 & 3.40423 & 1.84536 \\ \mathrm{C} & 1.27755 & 2.59824 & 1.04729\end{array}$

$1 c-D I H Y-A$

SCF Energy = $\quad-1409.204157$ hartree

Total Enthalpy $=-1408.866284$ hartree

\begin{tabular}{|c|c|c|c|}
\hline $\mathrm{Rh}$ & -0.15463 & 1.04833 & 0.97794 \\
\hline 0 & -0.36990 & 0.97728 & 3.16336 \\
\hline C & 0.64993 & 0.87782 & 3.85722 \\
\hline $\mathrm{N}$ & 1.90349 & 0.83682 & 3.35427 \\
\hline C & 2.22339 & 1.08619 & 1.98161 \\
\hline C & 1.88678 & 2.30711 & 1.43128 \\
\hline $\mathrm{H}$ & 2.25335 & 2.54291 & 0.43834 \\
\hline $\mathrm{H}$ & 2.66799 & 0.66312 & 3.99790 \\
\hline $\mathrm{H}$ & 0.57847 & 0.78554 & 4.94689 \\
\hline $\mathrm{P}$ & -0.24306 & 1.15469 & -1.28291 \\
\hline $\mathrm{P}$ & -0.31035 & -1.40428 & 0.69902 \\
\hline C & -0.46132 & -0.54285 & -1.93419 \\
\hline C & -0.49925 & -1.60136 & -1.11637 \\
\hline $\mathrm{H}$ & -1.68267 & 0.97139 & 0.80611 \\
\hline $\mathrm{H}$ & -0.61045 & 2.55269 & 0.92026 \\
\hline C & -1.63219 & 2.10685 & -2.01364 \\
\hline C & 1.23574 & 1.80444 & -2.16742 \\
\hline C & -1.88539 & -2.08309 & 1.38265 \\
\hline C & 0.89129 & -2.74997 & 1.11935 \\
\hline $\mathrm{H}$ & -1.52735 & 3.15917 & -1.73770 \\
\hline $\mathrm{H}$ & 1.10113 & 1.71026 & -3.24932 \\
\hline $\mathrm{H}$ & -2.05985 & -3.10903 & 1.04582 \\
\hline $\mathrm{H}$ & 1.06090 & -2.76297 & 2.19998 \\
\hline $\mathrm{H}$ & -0.58170 & -0.66041 & -3.01164 \\
\hline $\mathrm{H}$ & -0.64544 & -2.60349 & -1.52097 \\
\hline $\mathrm{H}$ & 1.84547 & -2.58712 & 0.61342 \\
\hline $\mathrm{H}$ & 0.49868 & -3.72637 & 0.81948 \\
\hline $\mathrm{H}$ & -1.83031 & -2.06988 & 2.47499 \\
\hline $\mathrm{H}$ & -2.72049 & -1.44991 & 1.07641 \\
\hline $\mathrm{H}$ & -2.57863 & 1.73817 & -1.61308 \\
\hline $\mathrm{H}$ & -1.63378 & 2.01554 & -3.10380 \\
\hline $\mathrm{H}$ & 1.36866 & 2.86273 & -1.92543 \\
\hline $\mathrm{H}$ & 2.13153 & 1.25110 & -1.87600 \\
\hline $\mathrm{H}$ & 3.36718 & 0.35450 & 0.30907 \\
\hline $\mathrm{H}$ & 4.22019 & 0.33703 & 1.85123 \\
\hline $\mathrm{H}$ & 2.98856 & -0.88736 & 1.52728 \\
\hline C & 3.24416 & 0.16201 & 1.37657 \\
\hline $\mathrm{N}$ & 1.18921 & 4.33328 & 2.89177 \\
\hline C & 1.46622 & 3.42087 & 2.22618 \\
\hline
\end{tabular}

1c-DIHY-C

$\mathrm{SCF}$ Energy $\quad=-1409.213315$ hartree

Total Enthalpy $=-1408.875580$ hartree 
Supporting Information for Donoghue et al.: Ligand and Substrate Effects on the Mechanisms of the Rhodium...

\begin{tabular}{|c|c|c|c|}
\hline $\mathrm{Rh}$ & 0.50721 & -0.63254 & 0.95194 \\
\hline 0 & 0.40374 & -0.70442 & 3.13987 \\
\hline C & 1.49675 & -0.70394 & 3.72324 \\
\hline $\mathrm{N}$ & 2.67336 & -0.51938 & 3.09173 \\
\hline $\mathrm{C}$ & 2.78352 & -0.12542 & 1.71690 \\
\hline $\mathrm{C}$ & 2.21168 & 1.07739 & 1.31994 \\
\hline $\mathrm{H}$ & 2.44416 & 1.46041 & 0.33242 \\
\hline $\mathrm{H}$ & 3.53030 & -0.65295 & 3.61731 \\
\hline $\mathrm{H}$ & 1.55759 & -0.87605 & 4.80336 \\
\hline $\mathrm{P}$ & 0.41409 & -0.84411 & -1.30716 \\
\hline $\mathrm{P}$ & -1.36553 & 0.94909 & 0.68962 \\
\hline $\mathrm{C}$ & -1.05627 & 0.06213 & -1.91509 \\
\hline $\mathrm{C}$ & -1.80399 & 0.80026 & -1.08641 \\
\hline $\mathrm{H}$ & -0.62792 & -1.67657 & 0.86485 \\
\hline $\mathrm{H}$ & 1. 28874 & -2.01025 & 0.95600 \\
\hline $\mathrm{C}$ & 1.80273 & -0.16181 & -2.30781 \\
\hline C & 0.21155 & -2.54683 & -1.96195 \\
\hline C & -1.37040 & 2.77349 & 0.96509 \\
\hline $\mathrm{C}$ & -2.87544 & 0.39499 & 1.58891 \\
\hline $\mathrm{H}$ & 2.71328 & -0.73533 & -2.11567 \\
\hline $\mathrm{H}$ & 0.07029 & -2.53090 & -3.04667 \\
\hline $\mathrm{H}$ & -2.35166 & 3.19060 & 0.71854 \\
\hline $\mathrm{H}$ & -2.70063 & 0.51063 & 2.66242 \\
\hline $\mathrm{H}$ & -2.67652 & 1.33685 & -1.46021 \\
\hline $\mathrm{H}$ & -1.30204 & -0.02478 & -2.97394 \\
\hline $\mathrm{H}$ & 1.97555 & 0.88765 & -2.05855 \\
\hline $\mathrm{H}$ & 1.56709 & -0.23391 & -3.37416 \\
\hline $\mathrm{H}$ & 1.10350 & -3.13104 & -1.72205 \\
\hline $\mathrm{H}$ & -0.64947 & -3.02027 & -1.48614 \\
\hline $\mathrm{H}$ & -3.06301 & -0.66071 & 1.38249 \\
\hline $\mathrm{H}$ & -3.74924 & 0.98779 & 1.30326 \\
\hline $\mathrm{H}$ & -1.13907 & 2.98523 & 2.01167 \\
\hline $\mathrm{H}$ & -0.60928 & 3.25341 & 0.34571 \\
\hline $\mathrm{H}$ & 3.91187 & -0.47398 & -0.08223 \\
\hline $\mathrm{H}$ & 4.86238 & -0.48257 & 1.40008 \\
\hline $\mathrm{H}$ & 3.83559 & -1.87791 & 1.01122 \\
\hline $\mathrm{C}$ & 3.90250 & -0.79015 & 0.96146 \\
\hline $\mathrm{N}$ & 1.28129 & 2.78069 & 3.03462 \\
\hline $\mathrm{C}$ & 1.68304 & 2.01328 & 2.2572 \\
\hline
\end{tabular}

$1 c-D I H Y-t s-A$

SCF Energy $=-1409.186814$ hartree Total Enthalpy $=-1408.851301$ hartree Negative Frequencies: $-774.33 \mathrm{~cm}^{-1}$

$\begin{array}{rrrr}\text { Rh } & -0.00079 & -0.00094 & 0.00061 \\ \mathrm{O} & 0.00032 & 0.00261 & 2.19598 \\ \mathrm{C} & 1.13960 & 0.00453 & 2.70574 \\ \mathrm{~N} & 2.25938 & 0.04869 & 1.97290 \\ \mathrm{C} & 2.22416 & 0.27942 & 0.54774 \\ \mathrm{C} & 1.70767 & 1.56804 & 0.14370 \\ \mathrm{H} & 2.00949 & 1.90536 & -0.84517 \\ \mathrm{H} & 3.15335 & 0.05540 & 2.45433 \\ \mathrm{H} & 1.26352 & -0.04600 & 3.79238 \\ \mathrm{P} & -0.38218 & -0.09604 & -2.23941\end{array}$


Supporting Information for Donoghue et al.: Ligand and Substrate Effects on the Mechanisms of the Rhodium...

$\begin{array}{lrrr}\mathrm{P} & -0.43082 & -2.30959 & 0.01422 \\ \mathrm{C} & -0.87095 & -1.81167 & -2.65259 \\ \mathrm{C} & -0.89600 & -2.75151 & -1.70100 \\ \mathrm{H} & -1.56828 & 0.19376 & -0.04023 \\ \mathrm{H} & 0.16143 & 1.63784 & -0.11521 \\ \mathrm{C} & -1.72951 & 0.96032 & -2.90235 \\ \mathrm{C} & 1.03023 & 0.26277 & -3.36756 \\ \mathrm{C} & -1.86844 & -2.78134 & 1.05619 \\ \mathrm{C} & 0.86463 & -3.53961 & 0.46859 \\ \mathrm{H} & -1.47877 & 2.00971 & -2.72442 \\ \mathrm{H} & 0.73384 & 0.10343 & -4.40880 \\ \mathrm{H} & -2.09140 & -3.84859 & 0.96883 \\ \mathrm{H} & 1.16102 & -3.37679 & 1.50860 \\ \mathrm{H} & -1.14439 & -2.03596 & -3.68362 \\ \mathrm{H} & -1.18934 & -3.77628 & -1.92805 \\ \mathrm{H} & 1.74133 & -3.41496 & -0.17093 \\ \mathrm{H} & 0.48888 & -4.56229 & 0.36729 \\ \mathrm{H} & -1.63581 & -2.54181 & 2.09731 \\ \mathrm{H} & -2.73940 & -2.19460 & 0.75752 \\ \mathrm{H} & -2.65933 & 0.73678 & -2.37611 \\ \mathrm{H} & -1.86031 & 0.79852 & -3.97640 \\ \mathrm{H} & 1.33621 & 1.30712 & -3.25498 \\ \mathrm{H} & 1.87680 & -0.38683 & -3.13624 \\ \mathrm{H} & 3.30092 & -0.14535 & -1.27382 \\ \mathrm{H} & 4.32717 & -0.04467 & 0.15405 \\ \mathrm{H} & 3.31962 & -1.47518 & -0.10260 \\ \mathrm{C} & 3.34705 & -0.38801 & -0.21034 \\ \mathrm{~N} & 1.63472 & 3.48466 & 1.90928 \\ \mathrm{C} & 1.64538 & 2.63865 & 1.11370\end{array}$

1c-DIHY-ts-C

SCF Energy = $=-1409.200632$ hartree Total Enthalpy $=-1408.864997$ hartree Negative Frequencies: $-732.79 \mathrm{~cm}^{-1}$

$\begin{array}{rrrr}\text { Rh } & 0.00020 & 0.00041 & -0.00175 \\ \mathrm{O} & 0.00198 & -0.00696 & 2.19229 \\ \mathrm{C} & 1.13394 & -0.00641 & 2.70306 \\ \mathrm{~N} & 2.26475 & 0.06861 & 1.98099 \\ \mathrm{C} & 2.26367 & 0.34950 & 0.56021 \\ \mathrm{C} & 1.66193 & 1.59624 & 0.16126 \\ \mathrm{H} & 1.92541 & 1.97462 & -0.82086 \\ \mathrm{H} & 3.15788 & 0.01755 & 2.45679 \\ \mathrm{H} & 1.25920 & -0.09260 & 3.78746 \\ \mathrm{P} & -0.22760 & -0.29750 & -2.24113 \\ \mathrm{P} & -2.04035 & 1.16285 & -0.12367 \\ \mathrm{C} & -1.87611 & 0.33037 & -2.73201 \\ \mathrm{C} & -2.65231 & 0.94793 & -1.83467 \\ \mathrm{H} & -0.85818 & -1.32043 & 0.01659 \\ \mathrm{H} & 1.38327 & -0.91104 & 0.08456 \\ \mathrm{C} & 0.93931 & 0.60017 & -3.34841 \\ \mathrm{C} & -0.17001 & -2.02557 & -2.85623 \\ \mathrm{C} & -2.10310 & 2.97390 & 0.18261 \\ \mathrm{C} & -3.33350 & 0.46059 & 0.97335 \\ \mathrm{H} & 1.95317 & 0.21438 & -3.21007 \\ \mathrm{H} & -0.35627 & -2.06082 & -3.93383\end{array}$


Supporting Information for Donoghue et al.: Ligand and Substrate Effects on the Mechanisms of the Rhodium...

$\begin{array}{rrrr}\mathrm{H} & -3.13721 & 3.33107 & 0.16704 \\ \mathrm{H} & -3.01125 & 0.58645 & 2.01068 \\ \mathrm{H} & -3.63251 & 1.33765 & -2.10833 \\ \mathrm{H} & -2.19429 & 0.19270 & -3.76555 \\ \mathrm{H} & 0.92783 & 1.66841 & -3.12120 \\ \mathrm{H} & 0.65518 & 0.45635 & -4.39535 \\ \mathrm{H} & 0.81690 & -2.44761 & -2.64844 \\ \mathrm{H} & -0.91786 & -2.62279 & -2.33120 \\ \mathrm{H} & -3.44506 & -0.60668 & 0.77199 \\ \mathrm{H} & -4.29111 & 0.96920 & 0.82893 \\ \mathrm{H} & -1.65465 & 3.19114 & 1.15617 \\ \mathrm{H} & -1.52467 & 3.49977 & -0.58027 \\ \mathrm{H} & 3.44526 & 0.03328 & -1.21569 \\ \mathrm{H} & 4.37852 & 0.42750 & 0.22666 \\ \mathrm{H} & 3.69146 & -1.20643 & 0.03401 \\ \mathrm{C} & 3.51618 & -0.14229 & -0.14106 \\ \mathrm{~N} & 0.93232 & 3.37005 & 1.89877 \\ \mathrm{C} & 1.27229 & 2.57614 & 1.11484\end{array}$

1c-ALHY - A

SCF Energy = $\quad-1409.233826$ hartree

Total Enthalpy $=-1408.892952$ hartree

$\begin{array}{rrrr}\mathrm{Rh} & 0.78873 & -0.16554 & 0.90793 \\ \mathrm{O} & 0.80043 & -0.33995 & 3.02259 \\ \mathrm{C} & 1.98051 & -0.40410 & 3.46479 \\ \mathrm{~N} & 3.03092 & -0.41406 & 2.66703 \\ \mathrm{C} & 2.86488 & -0.50014 & 1.19088 \\ \mathrm{C} & 3.89378 & 0.45407 & 0.54525 \\ \mathrm{H} & 3.87881 & 0.33261 & -0.54167 \\ \mathrm{H} & 3.96273 & -0.49701 & 3.07065 \\ \mathrm{H} & 2.15374 & -0.45329 & 4.54414 \\ \mathrm{P} & 0.66688 & 0.13015 & -1.37308 \\ \mathrm{P} & -1.61509 & 0.10196 & 0.84640 \\ \mathrm{C} & -1.08653 & 0.42470 & -1.82728 \\ \mathrm{C} & -2.05282 & 0.42389 & -0.90153 \\ \mathrm{H} & 1.02303 & 1.32493 & 0.95243 \\ \mathrm{H} & 3.65007 & 1.49788 & 0.76508 \\ \mathrm{C} & 1.53119 & 1.56427 & -2.13909 \\ \mathrm{C} & 1.15411 & -1.29232 & -2.44089 \\ \mathrm{C} & -2.30349 & 1.52438 & 1.78963 \\ \mathrm{C} & -2.69229 & -1.31179 & 1.33924 \\ \mathrm{H} & 2.61376 & 1.43912 & -2.06453 \\ \mathrm{H} & 0.86753 & -1.10083 & -3.47962 \\ \mathrm{H} & -3.38113 & 1.62517 & 1.63163 \\ \mathrm{H} & -2.52575 & -1.52826 & 2.39833 \\ \mathrm{H} & -3.09137 & 0.60527 & -1.17873 \\ \mathrm{H} & -1.31047 & 0.60689 & -2.87898 \\ \mathrm{H} & 1.24863 & 2.48403 & -1.62235 \\ \mathrm{H} & 1.26253 & 1.64866 & -3.19634 \\ \mathrm{H} & 2.23664 & -1.43808 & -2.40010 \\ \mathrm{H} & 0.66293 & -2.20681 & -2.09966 \\ \mathrm{H} & -2.43471 & -2.20045 & 0.75759 \\ \mathrm{H} & -3.75006 & -1.07748 & 1.18684 \\ \mathrm{H} & -2.11088 & 1.36064 & 2.85365 \\ \mathrm{H} & -1.80303 & 2.44654 & 1.48574\end{array}$


Supporting Information for Donoghue et al.: Ligand and Substrate Effects on the Mechanisms of the Rhodium...

$\begin{array}{rrrr}\mathrm{C} & 3.10147 & -1.96334 & 0.77721 \\ \mathrm{H} & 3.09401 & -2.07444 & -0.30994 \\ \mathrm{H} & 4.07159 & -2.33348 & 1.13731 \\ \mathrm{H} & 2.33418 & -2.62986 & 1.19215 \\ \mathrm{~N} & 6.29021 & -0.07608 & 1.48875 \\ \mathrm{C} & 5.25908 & 0.18680 & 1.02364\end{array}$

1c-ALHY-C

SCF Energy = $\quad-1409.248017$ hartree

Total Enthalpy $=-1408.906298$ hartree

$\begin{array}{rrrr}\mathrm{Rh} & 0.70303 & 0.09999 & 0.76697 \\ \mathrm{O} & 0.66870 & -0.14281 & 2.90779 \\ \mathrm{C} & 1.63051 & 0.01771 & 3.68682 \\ \mathrm{~N} & 2.92303 & 0.07921 & 3.37505 \\ \mathrm{C} & 3.56810 & -0.12847 & 2.05949 \\ \mathrm{C} & 2.79740 & 0.52727 & 0.90631 \\ \mathrm{H} & 3.35628 & 0.37733 & -0.01882 \\ \mathrm{H} & 3.55198 & 0.21487 & 4.15682 \\ \mathrm{H} & 1.42006 & 0.12708 & 4.75834 \\ \mathrm{P} & -1.66482 & -0.21894 & 0.69322 \\ \mathrm{P} & 0.55743 & 0.33658 & -1.49361 \\ \mathrm{C} & -2.12363 & -0.20025 & -1.08000 \\ \mathrm{C} & -1.17830 & 0.03358 & -1.99842 \\ \mathrm{H} & 0.93965 & -1.37173 & 0.47661 \\ \mathrm{H} & 3.58682 & -1.20685 & 1.85989 \\ \mathrm{C} & -2.31310 & -1.81258 & 1.34509 \\ \mathrm{C} & -2.75031 & 1.05060 & 1.47193 \\ \mathrm{C} & 1.54939 & -0.78775 & -2.55563 \\ \mathrm{C} & 0.93291 & 2.02132 & -2.12891 \\ \mathrm{H} & -2.09767 & -1.86210 & 2.41607 \\ \mathrm{H} & -3.80772 & 0.83638 & 1.29011 \\ \mathrm{H} & 1.33770 & -0.60516 & -3.61340 \\ \mathrm{H} & 1.99479 & 2.23916 & -1.99022 \\ \mathrm{H} & -3.15984 & -0.36779 & -1.37400 \\ \mathrm{H} & -1.41518 & 0.06391 & -3.06217 \\ \mathrm{H} & 0.35696 & 2.76387 & -1.57302 \\ \mathrm{H} & 0.68965 & 2.08881 & -3.19354 \\ \mathrm{H} & 2.61363 & -0.61520 & -2.37454 \\ \mathrm{H} & 1.31732 & -1.82673 & -2.31236 \\ \mathrm{H} & -1.80985 & -2.64685 & 0.85138 \\ \mathrm{H} & -3.39315 & -1.89595 & 1.19336 \\ \mathrm{H} & -2.56689 & 1.05221 & 2.55006 \\ \mathrm{H} & -2.50712 & 2.04033 & 1.07839 \\ \mathrm{H} & 5.54927 & 0.18773 & 1.24025 \\ \mathrm{H} & 5.02852 & 1.46256 & 2.35679 \\ \mathrm{H} & 5.55269 & -0.12339 & 2.97676 \\ \mathrm{C} & 5.01084 & 0.38394 & 2.17044 \\ \mathrm{~N} & 1.84684 & 2.91942 & 1.23020 \\ \mathrm{C} & 2.41359 & 1.90230 & 1.09323 \\ & & & \\ & & & \end{array}$

\section{ZDMP Ligand, Substrate d}

$1 d-S Q P L$ 
Supporting Information for Donoghue et al.: Ligand and Substrate Effects on the Mechanisms of the Rhodium...

$\begin{array}{lll}\text { SCF Energy } & =-1408.042464 & \text { hartree } \\ \text { Total Enthalpy } & =-1407.721712 \text { hartree }\end{array}$

$\begin{array}{rrrr}\mathrm{Rh} & 1.51837 & 4.44413 & 1.60946 \\ \mathrm{O} & 2.34905 & 4.07874 & -0.38292 \\ \mathrm{C} & 2.45998 & 2.85880 & -0.61149 \\ \mathrm{~N} & 2.01564 & 1.92892 & 0.23937 \\ \mathrm{C} & 1.21586 & 2.26752 & 1.40572 \\ \mathrm{C} & -0.02137 & 2.90183 & 1.13980 \\ \mathrm{H} & -0.30967 & 3.10589 & 0.11150 \\ \mathrm{H} & 2.14487 & 0.94742 & 0.01660 \\ \mathrm{H} & 2.93952 & 2.49771 & -1.52812 \\ \mathrm{P} & 0.86716 & 5.04749 & 3.73788 \\ \mathrm{P} & 3.08468 & 6.18316 & 1.85706 \\ \mathrm{C} & 2.05940 & 6.29726 & 4.37173 \\ \mathrm{C} & 3.00671 & 6.80016 & 3.57236 \\ \mathrm{C} & -0.74500 & 5.93519 & 3.84848 \\ \mathrm{C} & 0.76703 & 3.82999 & 5.11613 \\ \mathrm{C} & 2.84567 & 7.66217 & 0.78570 \\ \mathrm{C} & 4.83240 & 5.69559 & 1.55485 \\ \mathrm{H} & -1.55397 & 5.23055 & 3.64029 \\ \mathrm{H} & 0.59871 & 4.35770 & 6.06001 \\ \mathrm{H} & 3.61041 & 8.41893 & 0.98507 \\ \mathrm{H} & 4.91850 & 5.33549 & 0.52622 \\ \mathrm{H} & 1.94516 & 6.64005 & 5.40062 \\ \mathrm{H} & 3.69911 & 7.56872 & 3.91464 \\ \mathrm{H} & 5.11791 & 4.89160 & 2.23639 \\ \mathrm{H} & 5.50484 & 6.54773 & 1.69319 \\ \mathrm{H} & 2.91159 & 7.35302 & -0.26116 \\ \mathrm{H} & 1.85722 & 8.09295 & 0.96046 \\ \mathrm{H} & -0.77516 & 6.74423 & 3.11526 \\ \mathrm{H} & -0.88059 & 6.35220 & 4.85135 \\ \mathrm{H} & -0.07513 & 3.15568 & 4.94384 \\ \mathrm{H} & 1.69263 & 3.25541 & 5.18863 \\ \mathrm{H} & 0.80990 & 1.57404 & 3.40392 \\ \mathrm{H} & 1.36122 & 0.32273 & 2.29370 \\ \mathrm{H} & 2.52640 & 1.49632 & 2.93038 \\ \mathrm{C} & 1.49682 & 1.37514 & 2.58277 \\ \mathrm{~N} & -1.96590 & 2.84618 & 2.85065 \\ \mathrm{C} & -1.09502 & 2.86845 & 2.07713 \\ & & & \\ & & & \end{array}$

$1 d-M O L H-A$

SCF Energy $=-1409.220701$ hartree

Total Enthalpy $=-1408.884118$ hartree

$\begin{array}{rrrr}\text { Rh } & 3.29303 & 1.27617 & 1.28065 \\ \mathrm{O} & 3.17277 & 0.68113 & -0.81454 \\ \mathrm{C} & 1.98795 & 0.45144 & -1.14163 \\ \mathrm{~N} & 0.97671 & 0.62188 & -0.29129 \\ \mathrm{C} & 1.17173 & 1.30568 & 0.98544 \\ \mathrm{C} & 1.66869 & 2.66090 & 0.85253 \\ \mathrm{H} & 1.79985 & 3.07312 & -0.14514 \\ \mathrm{H} & 0.02892 & 0.42533 & -0.59725 \\ \mathrm{H} & 1.74616 & 0.08880 & -2.14635 \\ \mathrm{P} & 3.55460 & 1.65282 & 3.55562\end{array}$


Supporting Information for Donoghue et al.: Ligand and Substrate Effects on the Mechanisms of the Rhodium...

$\begin{array}{rrrr}\mathrm{P} & 4.43615 & -0.76637 & 1.72891 \\ \mathrm{C} & 4.59854 & 0.30506 & 4.23820 \\ \mathrm{C} & 4.96015 & -0.73550 & 3.47981 \\ \mathrm{H} & 5.17562 & 2.02330 & 0.91338 \\ \mathrm{H} & 4.69394 & 2.62527 & 0.83152 \\ \mathrm{C} & 4.46947 & 3.20361 & 3.93167 \\ \mathrm{C} & 2.15542 & 1.70438 & 4.75069 \\ \mathrm{C} & 5.98120 & -1.09714 & 0.78032 \\ \mathrm{C} & 3.49183 & -2.33903 & 1.53962 \\ \mathrm{H} & 3.83492 & 4.05203 & 3.65984 \\ \mathrm{H} & 2.56003 & 1.78687 & 5.76437 \\ \mathrm{H} & 6.43439 & -2.04889 & 1.07359 \\ \mathrm{H} & 3.21238 & -2.45909 & 0.48901 \\ \mathrm{H} & 4.88918 & 0.37235 & 5.28706 \\ \mathrm{H} & 5.56076 & -1.55037 & 3.88372 \\ \mathrm{H} & 2.58260 & -2.30133 & 2.14334 \\ \mathrm{H} & 4.09329 & -3.20139 & 1.84267 \\ \mathrm{H} & 5.73879 & -1.12973 & -0.28572 \\ \mathrm{H} & 6.69996 & -0.29072 & 0.94614 \\ \mathrm{H} & 5.39770 & 3.24562 & 3.35831 \\ \mathrm{H} & 4.70115 & 3.26037 & 4.99960 \\ \mathrm{H} & 1.53438 & 2.58006 & 4.54997 \\ \mathrm{H} & 1.55693 & 0.79422 & 4.68266 \\ \mathrm{H} & 0.18774 & 1.50821 & 2.89262 \\ \mathrm{H} & -0.87058 & 1.01455 & 1.58189 \\ \mathrm{H} & 0.25421 & -0.16316 & 2.28186 \\ \mathrm{C} & 0.13856 & 0.88689 & 2.00035 \\ \mathrm{~N} & 1.27462 & 4.40383 & 2.73061 \\ \mathrm{C} & 1.43464 & 3.63253 & 1.87082\end{array}$

$1 d-M O L H-C$

SCF Energy $\quad=-1409.217324$ hartree Total Enthalpy $=-1408.880133$ hartree

$\begin{array}{rrrr}\text { Rh } & 3.71818 & -0.06847 & 1.69947 \\ \mathrm{O} & 3.81443 & -0.86666 & -0.32725 \\ \mathrm{C} & 2.67119 & -1.10176 & -0.78574 \\ \mathrm{~N} & 1.56451 & -0.76582 & -0.12705 \\ \mathrm{C} & 1.63702 & 0.05563 & 1.07408 \\ \mathrm{C} & 2.33204 & 1.31489 & 0.87113 \\ \mathrm{H} & 2.68430 & 1.53758 & -0.13526 \\ \mathrm{H} & 0.65821 & -0.97479 & -0.53298 \\ \mathrm{H} & 2.55338 & -1.60256 & -1.75182 \\ \mathrm{P} & 3.79059 & 0.57783 & 3.92362 \\ \mathrm{P} & 5.91744 & 0.90434 & 1.61925 \\ \mathrm{C} & 5.33705 & 1.51659 & 4.22443 \\ \mathrm{C} & 6.25465 & 1.64086 & 3.25983 \\ \mathrm{H} & 4.49171 & -1.76583 & 2.18173 \\ \mathrm{H} & 3.71017 & -1.89620 & 2.22959 \\ \mathrm{C} & 2.50272 & 1.56460 & 4.78561 \\ \mathrm{C} & 3.96130 & -0.89059 & 5.02939 \\ \mathrm{C} & 6.07078 & 2.32107 & 0.44868 \\ \mathrm{C} & 7.40787 & -0.11242 & 1.24152 \\ \mathrm{H} & 1.56912 & 0.99774 & 4.82693 \\ \mathrm{H} & 4.12387 & -0.56767 & 6.06198 \\ \mathrm{H} & 7.05376 & 2.79504 & 0.52888\end{array}$


Supporting Information for Donoghue et al.: Ligand and Substrate Effects on the Mechanisms of the Rhodium...

$\begin{array}{rrrr}\mathrm{H} & 7.29381 & -0.54329 & 0.24259 \\ \mathrm{H} & 7.19012 & 2.17201 & 3.43565 \\ \mathrm{H} & 5.48709 & 1.94610 & 5.21516 \\ \mathrm{H} & 2.32309 & 2.51351 & 4.27908 \\ \mathrm{H} & 2.83834 & 1.75320 & 5.81033 \\ \mathrm{H} & 3.04630 & -1.48780 & 4.98599 \\ \mathrm{H} & 4.80364 & -1.50806 & 4.71145 \\ \mathrm{H} & 7.49657 & -0.92900 & 1.96215 \\ \mathrm{H} & 8.31976 & 0.49184 & 1.26544 \\ \mathrm{H} & 5.93795 & 1.95384 & -0.57301 \\ \mathrm{H} & 5.29511 & 3.06016 & 0.66173 \\ \mathrm{H} & 0.45898 & 0.60185 & 2.79175 \\ \mathrm{H} & -0.49704 & 0.12052 & 1.39793 \\ \mathrm{H} & 0.34277 & -1.11573 & 2.35277 \\ \mathrm{C} & 0.42391 & -0.09966 & 1.95758 \\ \mathrm{~N} & 1.74540 & 3.47185 & 2.18568 \\ \mathrm{C} & 1.99946 & 2.49726 & 1.60124\end{array}$

1d-MOLH-ts - A

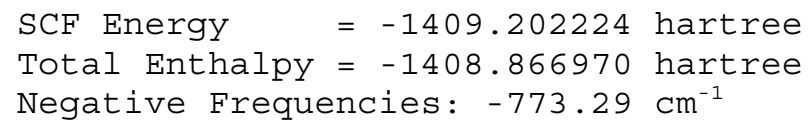

$\begin{array}{rrrr}\text { Rh } & -0.00004 & -0.00032 & 0.00057 \\ \mathrm{O} & 0.00355 & -0.00276 & 2.19439 \\ \mathrm{C} & 1.15162 & -0.00728 & 2.68563 \\ \mathrm{~N} & 2.25645 & 0.05557 & 1.93644 \\ \mathrm{C} & 2.20322 & 0.36214 & 0.52023 \\ \mathrm{C} & 1.54877 & 1.58639 & 0.20774 \\ \mathrm{H} & 1.18933 & 2.21491 & 1.01766 \\ \mathrm{H} & 3.16193 & 0.04139 & 2.39479 \\ \mathrm{H} & 1.29193 & -0.08429 & 3.76915 \\ \mathrm{P} & -0.21308 & -0.24617 & -2.28029 \\ \mathrm{P} & -0.20521 & -2.41585 & 0.05142 \\ \mathrm{C} & -0.81162 & -1.95349 & -2.58480 \\ \mathrm{C} & -0.78283 & -2.87953 & -1.62039 \\ \mathrm{H} & -1.59909 & 0.02108 & -0.03707 \\ \mathrm{H} & -1.04002 & 1.21679 & -0.09585 \\ \mathrm{C} & -1.43297 & 0.86042 & -3.08583 \\ \mathrm{C} & 1.25798 & -0.15073 & -3.38083 \\ \mathrm{C} & -1.52973 & -2.98519 & 1.19599 \\ \mathrm{C} & 1.17758 & -3.59199 & 0.39427 \\ \mathrm{H} & -1.06549 & 1.88841 & -3.02305 \\ \mathrm{H} & 0.95449 & -0.42167 & -4.39723 \\ \mathrm{H} & -1.67667 & -4.06722 & 1.13163 \\ \mathrm{H} & 1.54935 & -3.42175 & 1.40872 \\ \mathrm{H} & -1.14457 & -2.19584 & -3.59448 \\ \mathrm{H} & -1.09216 & -3.90596 & -1.81736 \\ \mathrm{H} & 1.99529 & -3.42711 & -0.31107 \\ \mathrm{H} & 0.84140 & -4.63039 & 0.31518 \\ \mathrm{H} & -1.24916 & -2.71845 & 2.21850 \\ \mathrm{H} & -2.46274 & -2.47152 & 0.95483 \\ \mathrm{H} & -2.39361 & 0.79440 & -2.57164 \\ \mathrm{H} & -1.55766 & 0.59027 & -4.13879 \\ \mathrm{H} & 1.65840 & 0.86509 & -3.38564 \\ \mathrm{H} & 2.02615 & -0.85033 & -3.04528\end{array}$


Supporting Information for Donoghue et al.: Ligand and Substrate Effects on the Mechanisms of the Rhodium...

$\begin{array}{rrrr}\mathrm{H} & 3.31629 & 0.10964 & -1.30540 \\ \mathrm{H} & 4.31187 & 0.16626 & 0.14170 \\ \mathrm{H} & 3.38363 & -1.29734 & -0.21960 \\ \mathrm{C} & 3.35924 & -0.20584 & -0.26286 \\ \mathrm{~N} & 2.01022 & 2.85604 & -2.00477 \\ \mathrm{C} & 1.80416 & 2.28724 & -1.00993\end{array}$

1d-MOLH-ts-C

SCF Energy $=-1409.207566$ hartree
Total Enthalpy $=-1408.872264$ hartree
Negative Frequencies: $-905.58 \mathrm{~cm}^{-1}$

\begin{tabular}{|c|c|c|c|}
\hline $\mathrm{Rh}$ & 0.00071 & 0.01294 & 0.00337 \\
\hline 0 & -0.00365 & 0.03245 & 2.19131 \\
\hline C & 1.15349 & 0.02521 & 2.66184 \\
\hline $\mathrm{N}$ & 2.23694 & 0.16819 & 1.89514 \\
\hline C & 2.13512 & 0.54199 & 0.49774 \\
\hline C & 1.41771 & 1.74822 & 0.25608 \\
\hline $\mathrm{H}$ & 1.04153 & 2.30749 & 1.10824 \\
\hline $\mathrm{H}$ & 3.15752 & 0.11230 & 2.31753 \\
\hline $\mathrm{H}$ & 1.31704 & -0.11064 & 3.73586 \\
\hline $\mathrm{P}$ & -0.18512 & -0.28212 & -2.27749 \\
\hline $\mathrm{P}$ & -2.00191 & 1.38411 & -0.24527 \\
\hline C & -1.66671 & 0.61975 & -2.86599 \\
\hline C & -2.44262 & 1.30420 & -2.01927 \\
\hline $\mathrm{H}$ & -0.82667 & -1.36137 & 0.08059 \\
\hline $\mathrm{H}$ & 0.36093 & -1.56249 & 0.08765 \\
\hline C & 1.14514 & 0.21370 & -3.44304 \\
\hline C & -0.51585 & -2.02832 & -2.75769 \\
\hline C & -1.93931 & 3.19532 & 0.10343 \\
\hline C & -3.50987 & 0.82873 & 0.65303 \\
\hline $\mathrm{H}$ & 2.03519 & -0.39408 & -3.26082 \\
\hline $\mathrm{H}$ & -0.71512 & -2.09469 & -3.83135 \\
\hline $\mathrm{H}$ & -2.88720 & 3.67701 & -0.15565 \\
\hline $\mathrm{H}$ & -3.32269 & 0.90211 & 1.72812 \\
\hline $\mathrm{H}$ & -3.32944 & 1.82955 & -2.37418 \\
\hline $\mathrm{H}$ & -1.89658 & 0.56600 & -3.93046 \\
\hline $\mathrm{H}$ & 1.39269 & 1.27000 & -3.33009 \\
\hline $\mathrm{H}$ & 0.80247 & 0.02764 & -4.46594 \\
\hline $\mathrm{H}$ & 0.35811 & -2.64021 & -2.51896 \\
\hline $\mathrm{H}$ & -1.37510 & -2.41119 & -2.20384 \\
\hline $\mathrm{H}$ & -3.71956 & -0.21531 & 0.41100 \\
\hline $\mathrm{H}$ & -4.37595 & 1.44671 & 0.39826 \\
\hline $\mathrm{H}$ & -1.74864 & 3.35013 & 1.16947 \\
\hline $\mathrm{H}$ & -1.13326 & 3.65596 & -0.47283 \\
\hline $\mathrm{H}$ & 3.20967 & 0.41843 & -1.36264 \\
\hline $\mathrm{H}$ & 4.23598 & 0.47707 & 0.06284 \\
\hline $\mathrm{H}$ & 3.37374 & -1.02352 & -0.33258 \\
\hline C & 3.29713 & 0.06573 & -0.33500 \\
\hline $\mathrm{N}$ & 1.73104 & 3.17802 & -1.88057 \\
\hline C & 1.60660 & 2.52845 & -0.92128 \\
\hline
\end{tabular}

1d-DIHY-A

SCF Energy = $\quad-1409.210786$ hartree

Total Enthalpy $=-1408.873051$ hartree 
Supporting Information for Donoghue et al.: Ligand and Substrate Effects on the Mechanisms of the Rhodium...

\begin{tabular}{rrrr}
$\mathrm{Rh}$ & 4.30493 & 2.22173 & 2.55788 \\
$\mathrm{O}$ & 4.29385 & 0.77197 & 0.88960 \\
$\mathrm{C}$ & 3.52087 & 0.04057 & 0.27549 \\
$\mathrm{~N}$ & 2.18028 & -0.10708 & 0.48517 \\
$\mathrm{C}$ & 1.36147 & 0.53318 & 1.43876 \\
$\mathrm{C}$ & 1.50800 & 1.85718 & 1.69675 \\
$\mathrm{H}$ & 2.17283 & 2.48077 & 1.10373 \\
$\mathrm{H}$ & 1.75291 & -0.87406 & -0.02225 \\
$\mathrm{H}$ & 3.89810 & -0.60823 & -0.52626 \\
$\mathrm{P}$ & 4.50005 & 3.82998 & 4.12507 \\
$\mathrm{P}$ & 4.77109 & 0.62320 & 4.38069 \\
$\mathrm{C}$ & 4.93607 & 3.04171 & 5.72186 \\
$\mathrm{C}$ & 5.05847 & 1.71330 & 5.82911 \\
$\mathrm{H}$ & 5.77810 & 2.49674 & 2.35852 \\
$\mathrm{H}$ & 4.20666 & 3.41309 & 1.51595 \\
$\mathrm{C}$ & 5.81997 & 5.07704 & 3.85421 \\
$\mathrm{C}$ & 3.00244 & 4.83153 & 4.48012 \\
$\mathrm{C}$ & 6.32432 & -0.36731 & 4.25943 \\
$\mathrm{C}$ & 3.55807 & -0.61053 & 5.04049 \\
$\mathrm{H}$ & 5.59177 & 5.64024 & 2.94605 \\
$\mathrm{H}$ & 3.19040 & 5.48811 & 5.33555 \\
$\mathrm{H}$ & 6.55739 & -0.87005 & 5.20265 \\
$\mathrm{H}$ & 3.39783 & -1.39227 & 4.29187 \\
$\mathrm{H}$ & 5.09897 & 3.69252 & 6.58144 \\
$\mathrm{H}$ & 5.32401 & 1.25923 & 6.78448 \\
$\mathrm{H}$ & 2.60325 & -0.12080 & 5.24795 \\
$\mathrm{H}$ & 3.92408 & -1.08029 & 5.95867 \\
$\mathrm{H}$ & 6.20385 & -1.12103 & 3.47591 \\
$\mathrm{H}$ & 7.15452 & 0.28537 & 3.98055 \\
$\mathrm{H}$ & 6.78233 & 4.57824 & 3.72258 \\
$\mathrm{H}$ & 5.87781 & 5.76509 & 4.70270 \\
$\mathrm{H}$ & 2.76951 & 5.44441 & 3.60541 \\
$\mathrm{H}$ & 2.14394 & 4.19169 & 4.69247 \\
$\mathrm{H}$ & -0.30456 & 0.19962 & 2.75900 \\
$\mathrm{H}$ & -0.31606 & -0.79420 & 1.29589 \\
$\mathrm{H}$ & 0.80394 & -1.18551 & 2.60432 \\
$\mathrm{C}$ & 0.32958 & -0.35609 & 2.06786 \\
$\mathrm{~N}$ & 0.15151 & 3.06606 & 3.53969 \\
$\mathrm{C}$ & 0.74027 & 2.51421 & 2.70016 \\
& & & \\
\hline & & &
\end{tabular}

1d-DIHY-C

$\mathrm{SCF}$ Energy $\quad=-1409.216904$ hartree

Total Enthalpy $=-1408.879035$ hartree

$\begin{array}{rrrr}\mathrm{Rh} & 4.47375 & 1.40457 & 2.21058 \\ \mathrm{O} & 4.91692 & 0.34196 & 0.30445 \\ \mathrm{C} & 4.28211 & -0.68612 & 0.05039 \\ \mathrm{~N} & 3.11573 & -1.02934 & 0.65186 \\ \mathrm{C} & 2.38050 & -0.20007 & 1.53904 \\ \mathrm{C} & 2.15101 & 1.10538 & 1.17779 \\ \mathrm{H} & 2.45449 & 1.46380 & 0.20065 \\ \mathrm{H} & 2.78233 & -1.97749 & 0.51889 \\ \mathrm{H} & 4.64961 & -1.40982 & -0.68711 \\ \mathrm{P} & 4.33415 & 2.33704 & 4.26790 \\ \mathrm{P} & 4.59557 & 3.71174 & 1.36538\end{array}$


Supporting Information for Donoghue et al.: Ligand and Substrate Effects on the Mechanisms of the Rhodium...

$\begin{array}{lrrr}\mathrm{C} & 4.45791 & 4.15565 & 4.09418 \\ \mathrm{C} & 4.57435 & 4.72968 & 2.89135 \\ \mathrm{H} & 5.96786 & 1.52207 & 2.51121 \\ \mathrm{H} & 4.68632 & 0.03491 & 2.98750 \\ \mathrm{C} & 2.80154 & 2.05968 & 5.24323 \\ \mathrm{C} & 5.67961 & 1.89786 & 5.43900 \\ \mathrm{C} & 3.32106 & 4.53026 & 0.30557 \\ \mathrm{C} & 6.17741 & 4.17799 & 0.53961 \\ \mathrm{H} & 2.70600 & 0.99335 & 5.46528 \\ \mathrm{H} & 5.57670 & 2.46246 & 6.37040 \\ \mathrm{H} & 3.53715 & 5.59621 & 0.18527 \\ \mathrm{H} & 6.23165 & 3.67461 & -0.42991 \\ \mathrm{H} & 4.65074 & 5.81367 & 2.80148 \\ \mathrm{H} & 4.44067 & 4.75430 & 5.00544 \\ \mathrm{H} & 1.92663 & 2.39566 & 4.68369 \\ \mathrm{H} & 2.86062 & 2.61108 & 6.18692 \\ \mathrm{H} & 5.62658 & 0.82831 & 5.65681 \\ \mathrm{H} & 6.64978 & 2.11247 & 4.98664 \\ \mathrm{H} & 7.02099 & 3.84364 & 1.14741 \\ \mathrm{H} & 6.24430 & 5.25878 & 0.38441 \\ \mathrm{H} & 3.31947 & 4.06449 & -0.68470 \\ \mathrm{H} & 2.33131 & 4.41688 & 0.75441 \\ \mathrm{H} & 1.19510 & -0.21786 & 3.33273 \\ \mathrm{H} & 0.93881 & -1.59666 & 2.26264 \\ \mathrm{H} & 2.41541 & -1.51507 & 3.24149 \\ \mathrm{C} & 1.70205 & -0.91852 & 2.66875 \\ \mathrm{~N} & 0.61055 & 2.68608 & 2.53122 \\ \mathrm{C} & 1.29518 & 1.96194 & 1.92831\end{array}$

$1 d-D I H Y-t s-A$

SCF Energy $\quad=-1409.190925$ hartree

Total Enthalpy $=-1408.855230$ hartree Negative Frequencies: $-800.75 \mathrm{~cm}^{-1}$

$\begin{array}{rrrr}\mathrm{Rh} & -0.00107 & 0.01138 & -0.00492 \\ \mathrm{O} & -0.00901 & 0.02771 & 2.20123 \\ \mathrm{C} & 1.13172 & 0.01516 & 2.71141 \\ \mathrm{~N} & 2.24986 & 0.09409 & 1.98254 \\ \mathrm{C} & 2.21489 & 0.34709 & 0.56000 \\ \mathrm{C} & 1.64151 & 1.63136 & 0.21790 \\ \mathrm{H} & 1.47764 & 2.32573 & 1.04224 \\ \mathrm{H} & 3.14754 & 0.04724 & 2.45458 \\ \mathrm{H} & 1.25582 & -0.07335 & 3.79609 \\ \mathrm{P} & -0.36120 & -0.14583 & -2.25785 \\ \mathrm{P} & -0.47047 & -2.28753 & 0.05477 \\ \mathrm{C} & -1.05902 & -1.81049 & -2.57719 \\ \mathrm{C} & -1.09038 & -2.73013 & -1.60697 \\ \mathrm{H} & -1.57056 & 0.19505 & -0.08125 \\ \mathrm{H} & 0.10522 & 1.65759 & -0.04855 \\ \mathrm{C} & -1.55277 & 1.04125 & -2.99028 \\ \mathrm{C} & 1.07977 & -0.07798 & -3.40189 \\ \mathrm{C} & -1.83265 & -2.69129 & 1.21927 \\ \mathrm{C} & 0.82133 & -3.54957 & 0.42500 \\ \mathrm{H} & -1.14296 & 2.05146 & -2.90208 \\ \mathrm{H} & 0.74383 & -0.28567 & -4.42284 \\ \mathrm{H} & -2.09465 & -3.75213 & 1.17120\end{array}$


Supporting Information for Donoghue et al.: Ligand and Substrate Effects on the Mechanisms of the Rhodium...

$\begin{array}{rrrr}\mathrm{H} & 1.19842 & -3.38687 & 1.43866 \\ \mathrm{H} & -1.41454 & -2.03077 & -3.58379 \\ \mathrm{H} & -1.47434 & -3.73382 & -1.78744 \\ \mathrm{H} & 1.65106 & -3.45243 & -0.27884 \\ \mathrm{H} & 0.41265 & -4.56268 & 0.36180 \\ \mathrm{H} & -1.51249 & -2.44023 & 2.23374 \\ \mathrm{H} & -2.70573 & -2.08233 & 0.97654 \\ \mathrm{H} & -2.49727 & 0.99860 & -2.44501 \\ \mathrm{H} & -1.72017 & 0.81719 & -4.04816 \\ \mathrm{H} & 1.53626 & 0.91419 & -3.37463 \\ \mathrm{H} & 1.82189 & -0.82556 & -3.11338 \\ \mathrm{H} & 3.31432 & -0.02070 & -1.26013 \\ \mathrm{H} & 4.32859 & 0.08628 & 0.17318 \\ \mathrm{H} & 3.35502 & -1.36609 & -0.10615 \\ \mathrm{C} & 3.35956 & -0.27738 & -0.20097 \\ \mathrm{~N} & 2.41808 & 2.81593 & -1.96743 \\ \mathrm{C} & 2.07313 & 2.29034 & -0.99039\end{array}$

1d-DIHY-ts-C

$\mathrm{SCF}$ Energy $=-1409.202154$ hartree
Total Enthalpy $=-1408.866348$ hartree
Negative Frequencies: $-761.96 \mathrm{~cm}^{-1}$

$\begin{array}{rrrr}\text { Rh } & 0.00220 & -0.01097 & 0.00224 \\ \mathrm{O} & 0.01762 & -0.03198 & 2.23045 \\ \mathrm{C} & 1.15400 & -0.02222 & 2.73197 \\ \mathrm{~N} & 2.27072 & 0.19511 & 2.01711 \\ \mathrm{C} & 2.22590 & 0.57451 & 0.61860 \\ \mathrm{C} & 1.43953 & 1.75060 & 0.35983 \\ \mathrm{H} & 1.01211 & 2.27272 & 1.20927 \\ \mathrm{H} & 3.17506 & 0.11601 & 2.46624 \\ \mathrm{H} & 1.29806 & -0.20657 & 3.80268 \\ \mathrm{P} & -0.15106 & -0.47800 & -2.22498 \\ \mathrm{P} & -2.06300 & 1.07419 & -0.25489 \\ \mathrm{C} & -1.77527 & 0.12679 & -2.81254 \\ \mathrm{C} & -2.59465 & 0.77467 & -1.97843 \\ \mathrm{H} & -0.75110 & -1.38842 & 0.02942 \\ \mathrm{H} & 1.49998 & -0.73879 & 0.10226 \\ \mathrm{C} & 1.06423 & 0.25079 & -3.39534 \\ \mathrm{C} & -0.13547 & -2.26307 & -2.65966 \\ \mathrm{C} & -2.13161 & 2.90548 & -0.07385 \\ \mathrm{C} & -3.42465 & 0.45244 & 0.80865 \\ \mathrm{H} & 2.05017 & -0.18602 & -3.21314 \\ \mathrm{H} & -0.26868 & -2.39566 & -3.73767 \\ \mathrm{H} & -3.12080 & 3.28894 & -0.34231 \\ \mathrm{H} & -3.17022 & 0.64755 & 1.85410 \\ \mathrm{H} & -3.56415 & 1.14537 & -2.31056 \\ \mathrm{H} & -2.04563 & -0.05515 & -3.85273 \\ \mathrm{H} & 1.12598 & 1.33327 & -3.27097 \\ \mathrm{H} & 0.76500 & 0.01136 & -4.42080 \\ \mathrm{H} & 0.82399 & -2.69408 & -2.36169 \\ \mathrm{H} & -0.93028 & -2.78175 & -2.12069 \\ \mathrm{H} & -3.52915 & -0.62620 & 0.67409 \\ \mathrm{H} & -4.36980 & 0.94897 & 0.56988 \\ \mathrm{H} & -1.92196 & 3.17092 & 0.96624 \\ \mathrm{H} & -1.37690 & 3.36590 & -0.71607\end{array}$


Supporting Information for Donoghue et al.: Ligand and Substrate Effects on the Mechanisms of the Rhodium...

$\begin{array}{rrrr}\mathrm{H} & 3.42220 & 0.56407 & -1.17281 \\ \mathrm{H} & 4.30661 & 0.96136 & 0.29633 \\ \mathrm{H} & 3.84113 & -0.73007 & -0.02425 \\ \mathrm{C} & 3.52541 & 0.31166 & -0.11689 \\ \mathrm{~N} & 1.74140 & 3.17951 & -1.77486 \\ \mathrm{C} & 1.62547 & 2.53512 & -0.80903\end{array}$

$1 d-A L H Y-A$

SCF Energy = -1409.235849 hartree

Total Enthalpy $=-1408.895047$ hartree

$\begin{array}{rrrr}\mathrm{Rh} & 3.55925 & 2.44817 & 2.87524 \\ \mathrm{O} & 1.81750 & 3.46070 & 2.19337 \\ \mathrm{C} & 1.57846 & 3.19645 & 0.98651 \\ \mathrm{~N} & 2.35851 & 2.40725 & 0.27016 \\ \mathrm{C} & 3.64437 & 1.88269 & 0.81165 \\ \mathrm{C} & 3.70165 & 0.41204 & 0.33461 \\ \mathrm{H} & 3.53709 & 0.35375 & -0.75471 \\ \mathrm{H} & 2.13112 & 2.26968 & -0.71203 \\ \mathrm{H} & 0.69808 & 3.62614 & 0.49796 \\ \mathrm{P} & 5.39026 & 1.39955 & 3.84311 \\ \mathrm{P} & 3.25265 & 3.41598 & 5.06428 \\ \mathrm{C} & 5.56989 & 2.06093 & 5.55094 \\ \mathrm{C} & 4.66943 & 2.89135 & 6.08796 \\ \mathrm{H} & 2.81894 & 1.17623 & 3.20660 \\ \mathrm{H} & 2.90883 & -0.17691 & 0.80651 \\ \mathrm{C} & 5.33293 & -0.41304 & 4.15774 \\ \mathrm{C} & 7.05404 & 1.68927 & 3.10984 \\ \mathrm{C} & 1.74881 & 2.91786 & 6.00164 \\ \mathrm{C} & 3.24032 & 5.25641 & 5.16788 \\ \mathrm{H} & 5.41334 & -0.95611 & 3.21528 \\ \mathrm{H} & 7.82738 & 1.36543 & 3.81367 \\ \mathrm{H} & 1.73214 & 3.37135 & 6.99699 \\ \mathrm{H} & 2.38447 & 5.63466 & 4.60191 \\ \mathrm{H} & 4.77200 & 3.24979 & 7.11218 \\ \mathrm{H} & 6.43178 & 1.71729 & 6.12458 \\ \mathrm{H} & 4.39398 & -0.66919 & 4.65335 \\ \mathrm{H} & 6.16936 & -0.70008 & 4.80251 \\ \mathrm{H} & 7.15191 & 1.10679 & 2.19123 \\ \mathrm{H} & 7.19451 & 2.75111 & 2.89317 \\ \mathrm{H} & 4.15553 & 5.66315 & 4.73046 \\ \mathrm{H} & 3.15857 & 5.59409 & 6.20542 \\ \mathrm{H} & 0.86605 & 3.24448 & 5.44479 \\ \mathrm{H} & 1.71618 & 1.83022 & 6.09756 \\ \mathrm{C} & 4.78173 & 2.72697 & 0.21353 \\ \mathrm{H} & 5.75958 & 2.32141 & 0.48022 \\ \mathrm{H} & 4.72902 & 2.76023 & -0.88546 \\ \mathrm{H} & 4.73698 & 3.76730 & 0.56158 \\ \mathrm{~N} & 6.02208 & -0.70427 & 0.81957 \\ \mathrm{C} & 4.98692 & -0.22718 & 0.59965 \\ & & & \\ & & & \end{array}$

1d-ALHY-C

SCF Energy $\quad=-1409.248041$ hartree

Total Enthalpy $=-1408.906342$ hartree 
Supporting Information for Donoghue et al.: Ligand and Substrate Effects on the Mechanisms of the Rhodium...

\begin{tabular}{|c|c|c|c|}
\hline $\mathrm{Rh}$ & 3.64967 & -0.52253 & 2.33079 \\
\hline O & 2.38383 & -2.20796 & 1.88211 \\
\hline C & 1.50501 & -2.25900 & 0.99729 \\
\hline $\mathrm{N}$ & 0.94580 & -1.23733 & 0.35316 \\
\hline C & 1.13182 & 0.21274 & 0.58291 \\
\hline C & 2.59657 & 0.59401 & 0.83616 \\
\hline $\mathrm{H}$ & 2.66323 & 1.67774 & 0.94472 \\
\hline $\mathrm{H}$ & 0.24676 & -1.48807 & -0.33509 \\
\hline $\mathrm{H}$ & 1.13101 & -3.24422 & 0.69040 \\
\hline $\mathrm{P}$ & 4.88846 & -1.86948 & 3.86724 \\
\hline $\mathrm{P}$ & 5.08945 & 1.14309 & 2.91595 \\
\hline $\mathrm{C}$ & 6.12002 & -0.75657 & 4.64154 \\
\hline C & 6.19722 & 0.51691 & 4.23609 \\
\hline $\mathrm{H}$ & 2.77203 & 0.00270 & 3.45317 \\
\hline $\mathrm{H}$ & 0.56892 & 0.48593 & 1.48396 \\
\hline C & 3.95098 & -2.60741 & 5.26773 \\
\hline C & 5.87515 & -3.27887 & 3.20660 \\
\hline C & 4.39441 & 2.70152 & 3.59709 \\
\hline C & 6.23093 & 1.69044 & 1.58145 \\
\hline $\mathrm{H}$ & 3.21845 & -3.31042 & 4.86123 \\
\hline $\mathrm{H}$ & 6.46145 & -3.75969 & 3.99544 \\
\hline $\mathrm{H}$ & 5.19651 & 3.38515 & 3.89089 \\
\hline $\mathrm{H}$ & 5.66515 & 2.20376 & 0.79983 \\
\hline $\mathrm{H}$ & 6.78556 & -1.13850 & 5.41581 \\
\hline $\mathrm{H}$ & 6.92443 & 1.20671 & 4.6651 \\
\hline $\mathrm{H}$ & 6.72017 & 0.82284 & 1.1338 \\
\hline $\mathrm{H}$ & 6.98636 & 2.37221 & 1.98363 \\
\hline $\mathrm{H}$ & 3.77767 & 3.18816 & 2.83670 \\
\hline $\mathrm{H}$ & 3.76939 & 2.47959 & 4.46471 \\
\hline $\mathrm{H}$ & 3.41594 & -1.82406 & 5.80926 \\
\hline $\mathrm{H}$ & 4.61405 & -3.13989 & 5.95579 \\
\hline $\mathrm{H}$ & 5.18808 & -4.01246 & 2.7753 \\
\hline $\mathrm{H}$ & 6.54526 & -2.92781 & 2.41829 \\
\hline $\mathrm{H}$ & 0.58458 & 2.02876 & -0.46665 \\
\hline $\mathrm{H}$ & 1.07924 & 0.70708 & -1.54030 \\
\hline $\mathrm{H}$ & -0.52418 & 0.68885 & -0.76307 \\
\hline $\mathrm{C}$ & 0.53138 & 0.94867 & -0.62349 \\
\hline $\mathrm{N}$ & 4.44189 & -0.46998 & -0.64519 \\
\hline $\mathrm{C}$ & 3.56129 & 0.08035 & -0.10092 \\
\hline
\end{tabular}

\section{DMPE Ligand, Substrate a}

\begin{tabular}{|c|c|c|c|}
\hline \multicolumn{2}{|c|}{ SCF Energy } & \multicolumn{2}{|c|}{-1369.954360 hartree } \\
\hline Total & 1 Enthalpy $=-$ & 9396 hart & \\
\hline $\mathrm{Rh}$ & 0.48878 & 0.74825 & 0.92276 \\
\hline 0 & 1.12381 & 0.51945 & 3.00388 \\
\hline $\mathrm{C}$ & 1.76175 & 1.50791 & 3.42105 \\
\hline $\mathrm{N}$ & 1.93291 & 2.61868 & 2.69617 \\
\hline $\mathrm{C}$ & 1.30224 & 2.75268 & 1.39694 \\
\hline $\mathrm{C}$ & -0.11251 & 2.77296 & 1.31655 \\
\hline $\mathrm{C}$ & 2.13540 & 3.27931 & 0.36126 \\
\hline $\mathrm{N}$ & 2.79199 & 3.69466 & -0.50696 \\
\hline
\end{tabular}


Supporting Information for Donoghue et al.: Ligand and Substrate Effects on the Mechanisms of the Rhodium...

$\begin{array}{rrrr}\mathrm{H} & -0.68566 & 2.77102 & 2.23991 \\ \mathrm{H} & -0.59768 & 3.23862 & 0.46746 \\ \mathrm{H} & 2.44650 & 3.40071 & 3.08719 \\ \mathrm{H} & 2.21079 & 1.50457 & 4.42020 \\ \mathrm{P} & 0.07458 & 0.79749 & -1.32851 \\ \mathrm{P} & -0.72886 & -1.25715 & 0.88712 \\ \mathrm{C} & -0.98638 & 2.13635 & -2.01682 \\ \mathrm{C} & 1.59851 & 0.86835 & -2.35377 \\ \mathrm{C} & -1.97495 & -1.43307 & 2.22462 \\ \mathrm{C} & 0.24447 & -2.82050 & 0.97191 \\ \mathrm{H} & -0.49872 & 3.10559 & -1.88213 \\ \mathrm{H} & 1.34673 & 0.78483 & -3.41596 \\ \mathrm{H} & -2.53366 & -2.36801 & 2.11721 \\ \mathrm{H} & 0.81755 & -2.83709 & 1.90286 \\ \mathrm{H} & 0.94753 & -2.87909 & 0.13713 \\ \mathrm{H} & -0.41725 & -3.69201 & 0.94554 \\ \mathrm{H} & -1.46371 & -1.43024 & 3.19085 \\ \mathrm{H} & -2.66972 & -0.59028 & 2.19753 \\ \mathrm{H} & -1.95777 & 2.15423 & -1.51610 \\ \mathrm{H} & -1.13937 & 1.97586 & -3.08826 \\ \mathrm{H} & 2.11798 & 1.81398 & -2.17734 \\ \mathrm{H} & 2.26793 & 0.04920 & -2.07860 \\ \mathrm{C} & -1.66096 & -1.33372 & -0.71917 \\ \mathrm{H} & -1.99950 & -2.35394 & -0.93037 \\ \mathrm{H} & -2.55447 & -0.71028 & -0.60132 \\ \mathrm{C} & -0.76369 & -0.79429 & -1.84241 \\ \mathrm{H} & -1.32589 & -0.63888 & -2.76935 \\ \mathrm{H} & 0.03485 & -1.50963 & -2.07076\end{array}$

$2 a-M O L H-A$

SCF Energy = $\quad-1371.130391$ hartree

Total Enthalpy $=-1370.799183$ hartree

$\begin{array}{rrrr}\mathrm{Rh} & 0.14339 & 1.11479 & 0.94742 \\ \mathrm{O} & 0.36116 & 1.00537 & 3.12730 \\ \mathrm{C} & 1.55825 & 0.87718 & 3.45614 \\ \mathrm{~N} & 2.54552 & 0.84790 & 2.55689 \\ \mathrm{C} & 2.25941 & 1.19538 & 1.16837 \\ \mathrm{C} & 1.79085 & 2.52753 & 0.90520 \\ \mathrm{C} & 3.01820 & 0.42075 & 0.22734 \\ \mathrm{~N} & 3.60737 & -0.22743 & -0.53996 \\ \mathrm{H} & 1.77218 & 3.22923 & 1.73359 \\ \mathrm{H} & 1.97797 & 2.96972 & -0.06605 \\ \mathrm{H} & 3.51043 & 0.77871 & 2.86313 \\ \mathrm{H} & 1.84313 & 0.77339 & 4.50879 \\ \mathrm{P} & -0.08968 & 0.95899 & -1.34087 \\ \mathrm{P} & -0.70208 & -1.15409 & 0.93490 \\ \mathrm{H} & -1.63525 & 1.78489 & 1.14263 \\ \mathrm{H} & -1.18711 & 2.44123 & 1.06250 \\ \mathrm{C} & -1.66448 & 1.70625 & -1.93224 \\ \mathrm{C} & 1.17269 & 1.65773 & -2.47559 \\ \mathrm{C} & -2.17906 & -1.63452 & 1.92308 \\ \mathrm{C} & 0.56123 & -2.40942 & 1.41535 \\ \mathrm{H} & -1.64659 & 2.78467 & -1.75491 \\ \mathrm{H} & 0.89674 & 1.42203 & -3.50818 \\ \mathrm{H} & -2.42560 & -2.69185 & 1.78557\end{array}$


Supporting Information for Donoghue et al.: Ligand and Substrate Effects on the Mechanisms of the Rhodium...

$\begin{array}{lrrr}\mathrm{H} & 0.84371 & -2.25985 & 2.46101 \\ \mathrm{H} & 1.45608 & -2.29527 & 0.79803 \\ \mathrm{H} & 0.16890 & -3.42477 & 1.30098 \\ \mathrm{H} & -1.97449 & -1.45160 & 2.98156 \\ \mathrm{H} & -3.03767 & -1.02341 & 1.63219 \\ \mathrm{H} & -2.51622 & 1.28310 & -1.39493 \\ \mathrm{H} & -1.79034 & 1.52515 & -3.00403 \\ \mathrm{H} & 1.21486 & 2.74451 & -2.36801 \\ \mathrm{H} & 2.15660 & 1.23351 & -2.26678 \\ \mathrm{C} & -1.13079 & -1.58562 & -0.82695 \\ \mathrm{H} & -1.07463 & -2.66793 & -0.98387 \\ \mathrm{H} & -2.17357 & -1.29199 & -0.99259 \\ \mathrm{C} & -0.19761 & -0.83947 & -1.79280 \\ \mathrm{H} & -0.52943 & -0.93930 & -2.83261 \\ \mathrm{H} & 0.82294 & -1.23537 & -1.73735\end{array}$

$2 \mathrm{a}-\mathrm{MOLH}-\mathrm{C}$

$\begin{array}{lll}\text { SCF Energy } & =-1371.134603 & \text { hartree } \\ \text { Total Enthalpy } & =-1370.803698 \text { hartree }\end{array}$

$\begin{array}{rrrr}\mathrm{Rh} & 0.69080 & -0.30130 & 0.95315 \\ \mathrm{O} & 0.65608 & -0.42023 & 3.14663 \\ \mathrm{C} & 1.80385 & -0.34765 & 3.63853 \\ \mathrm{~N} & 2.88880 & -0.12078 & 2.89573 \\ \mathrm{C} & 2.74048 & 0.20267 & 1.48670 \\ \mathrm{C} & 1.96328 & 1.36854 & 1.14636 \\ \mathrm{C} & 3.78961 & -0.29028 & 0.64873 \\ \mathrm{~N} & 4.62664 & -0.68777 & -0.05738 \\ \mathrm{H} & 1.63823 & 2.01386 & 1.95904 \\ \mathrm{H} & 2.19196 & 1.89176 & 0.22477 \\ \mathrm{H} & 3.80058 & -0.05850 & 3.33533 \\ \mathrm{H} & 1.95918 & -0.47220 & 4.71529 \\ \mathrm{P} & 0.66408 & -0.27079 & -1.34208 \\ \mathrm{P} & -1.61152 & 0.24212 & 0.75174 \\ \mathrm{H} & 0.10843 & -2.26230 & 1.06760 \\ \mathrm{H} & 0.88223 & -2.29100 & 1.03471 \\ \mathrm{C} & 1.98305 & 0.58890 & -2.28617 \\ \mathrm{C} & 0.66610 & -1.97355 & -2.04021 \\ \mathrm{C} & -1.98786 & 1.98125 & 1.22552 \\ \mathrm{C} & -2.85363 & -0.74187 & 1.68548 \\ \mathrm{H} & 2.96033 & 0.17517 & -2.02493 \\ \mathrm{H} & 0.57026 & -1.93848 & -3.12975 \\ \mathrm{H} & -3.04834 & 2.20890 & 1.07972 \\ \mathrm{H} & -2.63363 & -0.67128 & 2.75428 \\ \mathrm{H} & 1.98015 & 1.65941 & -2.06736 \\ \mathrm{H} & 1.81244 & 0.45129 & -3.35827 \\ \mathrm{H} & 1.60689 & -2.46760 & -1.78290 \\ \mathrm{H} & -0.15828 & -2.55875 & -1.62607 \\ \mathrm{H} & -2.79554 & -1.79359 & 1.39252 \\ \mathrm{H} & -3.86823 & -0.37349 & -1.50505 \\ \mathrm{H} & -1.73460 & 2.12721 & 2.27904 \\ \mathrm{H} & -1.38406 & 2.67094 & 0.63073 \\ \mathrm{C} & -0.92234 & 0.51011 & -1.93323 \\ \mathrm{H} & -0.76928 & 1.59481 & -1.89561 \\ \mathrm{H} & -1.09743 & 0.24620 & -10022 \\ \mathrm{C} & -2.10264 & & -1.03968\end{array}$


Supporting Information for Donoghue et al.: Ligand and Substrate Effects on the Mechanisms of the Rhodium...

$\begin{array}{rrrr}\mathrm{H} & -2.37625 & -0.94637 & -1.21596 \\ \mathrm{H} & -2.99049 & 0.70551 & -1.25122\end{array}$

$2 a-M O L H-t s-A$

SCF Energy = -1371.119829 hartree

Total Enthalpy $=-1370.790331$ hartree

Negative Frequencies: $-840.24 \mathrm{~cm}^{-1}$

$\begin{array}{rrrr}\mathrm{Rh} & 0.00000 & 0.00000 & 0.00000 \\ \mathrm{O} & 2.20780 & 0.00000 & 0.00000 \\ \mathrm{C} & 2.69252 & 1.14784 & 0.00000 \\ \mathrm{~N} & 1.93976 & 2.25653 & 0.04624 \\ \mathrm{C} & 0.52034 & 2.15510 & 0.32345 \\ \mathrm{C} & 0.11815 & 1.56586 & 1.54846 \\ \mathrm{C} & -0.32574 & 3.02678 & -0.43226 \\ \mathrm{~N} & -1.03900 & 3.69969 & -1.06258 \\ \mathrm{H} & 0.88289 & 1.30811 & 2.27297 \\ \mathrm{H} & -0.85904 & 1.78836 & 1.95641 \\ \mathrm{H} & 2.39022 & 3.16476 & 0.08406 \\ \mathrm{H} & 3.77689 & 1.29683 & -0.05104 \\ \mathrm{P} & -2.28434 & -0.00799 & -0.22144 \\ \mathrm{P} & 0.02460 & -0.14566 & -2.45125 \\ \mathrm{H} & -0.09132 & -1.59672 & 0.10274 \\ \mathrm{H} & -0.14203 & -1.03256 & 1.22403 \\ \mathrm{C} & -3.07650 & -1.62399 & 0.14003 \\ \mathrm{C} & -3.26296 & 1.20199 & 0.75139 \\ \mathrm{C} & 0.94555 & -1.56287 & -3.17460 \\ \mathrm{C} & 0.59665 & 1.29690 & -3.44843 \\ \mathrm{H} & -2.91303 & -1.88099 & 1.18959 \\ \mathrm{H} & -4.31924 & 1.11440 & 0.47782 \\ \mathrm{H} & 0.82631 & -1.60208 & -4.26155 \\ \mathrm{H} & 1.65553 & 1.48174 & -3.24704 \\ \mathrm{H} & 0.03249 & 2.19269 & -3.17808 \\ \mathrm{H} & 0.47430 & 1.09909 & -4.51794 \\ \mathrm{H} & 2.00716 & -1.45711 & -2.93533 \\ \mathrm{H} & 0.59075 & -2.49735 & -2.73333 \\ \mathrm{H} & -2.63276 & -2.40818 & -0.47727 \\ \mathrm{H} & -4.15197 & -1.57190 & -0.05457 \\ \mathrm{H} & -3.16226 & 0.99547 & -1.82000 \\ \mathrm{H} & -2.92973 & 2.22075 & 0.54133 \\ \mathrm{C} & -1.75377 & -0.39204 & -2.95125 \\ \mathrm{H} & -1.90925 & -0.07045 & -3.98638 \\ \mathrm{H} & -1.95910 & -1.46777 & -2.91337 \\ \mathrm{C} & -2.68088 & 0.37551 & -1.99612 \\ \mathrm{H} & -3.73573 & 0.14810 & -18901 \\ \mathrm{H} & -2.55190 & 1.45794 & \\ & & & \\ \mathrm{H} & -1314\end{array}$

$2 a-M O L H-t s-C$

SCF Energy = $\quad-1371.116496$ hartree

Total Enthalpy $=-1370.787205$ hartree Negative Frequencies: $-829.79 \mathrm{~cm}^{-1}$

$\begin{array}{rlll}\text { Rh } & 0.00000 & 0.00000 & 0.00000 \\ \mathrm{O} & 2.19412 & 0.00000 & 0.00000 \\ \mathrm{C} & 2.66771 & 1.15363 & 0.00000\end{array}$


Supporting Information for Donoghue et al.: Ligand and Substrate Effects on the Mechanisms of the Rhodium...

$\begin{array}{rrrr}\mathrm{N} & 1.90788 & 2.24569 & 0.13081 \\ \mathrm{C} & 0.49854 & 2.12789 & 0.45390 \\ \mathrm{C} & 0.13668 & 1.48936 & 1.66446 \\ \mathrm{C} & -0.33409 & 3.10762 & -0.18139 \\ \mathrm{~N} & -1.01091 & 3.91436 & -0.67645 \\ \mathrm{H} & 0.92416 & 1.20484 & 2.35363 \\ \mathrm{H} & -0.82935 & 1.70292 & 2.10536 \\ \mathrm{H} & 2.33186 & 3.16629 & 0.09794 \\ \mathrm{H} & 3.74485 & 1.31612 & -0.11270 \\ \mathrm{P} & -2.28934 & -0.10336 & -0.16878 \\ \mathrm{P} & -0.20504 & -1.94085 & 1.43940 \\ \mathrm{H} & 0.02414 & -0.99218 & -1.25506 \\ \mathrm{H} & 0.05128 & 0.27293 & -1.58685 \\ \mathrm{C} & -3.27943 & 1.40693 & 0.14803 \\ \mathrm{C} & -2.88532 & -0.69697 & -1.80024 \\ \mathrm{C} & -0.00797 & -1.76587 & 3.26428 \\ \mathrm{C} & 0.87940 & -3.36305 & 1.02115 \\ \mathrm{H} & -3.01929 & 2.18686 & -0.57105 \\ \mathrm{H} & -3.97551 & -0.78921 & -1.79693 \\ \mathrm{H} & -0.18178 & -2.72081 & 3.76993 \\ \mathrm{H} & 1.92256 & -3.07171 & 1.17033 \\ \mathrm{H} & -3.09331 & 1.78949 & 1.15430 \\ \mathrm{H} & -4.34322 & 1.16745 & 0.05278 \\ \mathrm{H} & -2.58672 & 0.01539 & -2.57323 \\ \mathrm{H} & -2.43790 & -1.66549 & -2.03454 \\ \mathrm{H} & 0.74811 & -3.62866 & -0.03043 \\ \mathrm{H} & 0.65631 & -4.23039 & 1.64997 \\ \mathrm{H} & 1.00820 & -1.43025 & 3.48917 \\ \mathrm{H} & -0.70805 & -1.02186 & 3.65282 \\ \mathrm{C} & -2.90749 & -1.34799 & 1.07303 \\ \mathrm{H} & -2.99530 & -0.82094 & 2.03029 \\ \mathrm{H} & -3.91646 & -1.67044 & 0.79198 \\ \mathrm{C} & -1.95005 & -2.54293 & 1.20063 \\ \mathrm{H} & -1.95877 & -3.14508 & 0.28513 \\ \mathrm{H} & -2.25147 & -3.19987 & \\ & & & 02358\end{array}$

$2 \mathrm{a}-\mathrm{DIHY}-\mathrm{A}$

SCF Energy $=-1371.126295$ hartree Total Enthalpy $=-1370.794232$ hartree

$\begin{array}{rrrr}\text { Rh } & -0.13116 & 1.06446 & 1.03161 \\ \mathrm{O} & -0.28736 & 0.86404 & 3.23801 \\ \mathrm{C} & 0.79213 & 0.70264 & 3.82733 \\ \mathrm{~N} & 1.98672 & 0.72342 & 3.20542 \\ \mathrm{C} & 2.10440 & 1.11118 & 1.82169 \\ \mathrm{C} & 1.71524 & 2.38891 & 1.43102 \\ \mathrm{C} & 2.92431 & 0.27115 & 1.00086 \\ \mathrm{~N} & 3.58986 & -0.41155 & 0.33156 \\ \mathrm{H} & 1.42107 & 3.10794 & 2.18580 \\ \mathrm{H} & 2.02972 & 2.78499 & 0.47476 \\ \mathrm{H} & 2.83158 & 0.58565 & 3.74891 \\ \mathrm{H} & 0.82268 & 0.51933 & 4.90770 \\ \mathrm{P} & -0.20582 & 1.16641 & -1.24401 \\ \mathrm{P} & -0.26507 & -1.38181 & 0.75069 \\ \mathrm{H} & -1.67206 & 1.02215 & 0.84158 \\ \mathrm{H} & -0.61315 & 2.57139 & 1.02605\end{array}$


Supporting Information for Donoghue et al.: Ligand and Substrate Effects on the Mechanisms of the Rhodium...

$\begin{array}{rrrr}\mathrm{C} & -1.38421 & 2.35697 & -1.98644 \\ \mathrm{C} & 1.39063 & 1.50640 & -2.09090 \\ \mathrm{C} & -1.93478 & -2.03247 & 1.17580 \\ \mathrm{C} & 0.87351 & -2.61442 & 1.51246 \\ \mathrm{H} & -1.07927 & 3.37532 & -1.73362 \\ \mathrm{H} & 1.26153 & 1.40328 & -3.17294 \\ \mathrm{H} & -2.01490 & -3.09792 & 0.93985 \\ \mathrm{H} & 0.76881 & -2.58314 & 2.60060 \\ \mathrm{H} & 1.90856 & -2.38780 & 1.24791 \\ \mathrm{H} & 0.62792 & -3.62433 & 1.16964 \\ \mathrm{H} & -2.11364 & -1.88751 & 2.24469 \\ \mathrm{H} & -2.70358 & -1.47971 & 0.63089 \\ \mathrm{H} & -2.38290 & 2.18689 & -1.57839 \\ \mathrm{H} & -1.40674 & 2.24724 & -3.07484 \\ \mathrm{H} & 1.71543 & 2.52895 & -1.88075 \\ \mathrm{H} & 2.16545 & 0.81388 & -1.75372 \\ \mathrm{C} & -0.74253 & -0.50310 & -1.86729 \\ \mathrm{H} & -1.83256 & -0.54021 & -1.76074 \\ \mathrm{H} & -0.52080 & -0.57557 & -2.93803 \\ \mathrm{C} & -0.07973 & -1.64592 & -1.08220 \\ \mathrm{H} & 0.99622 & -1.68460 & -1.28751 \\ \mathrm{H} & -0.50117 & -2.61357 & -1.37555\end{array}$

$2 a-D I H Y-C$

SCF Energy $\quad=-1371.122882$ hartree

Total Enthalpy $=-1370.790989$ hartree

$\begin{array}{rrrr}\mathrm{Rh} & 0.53425 & -0.60116 & 0.99187 \\ \mathrm{O} & 0.50796 & -0.67370 & 3.20733 \\ \mathrm{C} & 1.64049 & -0.68329 & 3.71298 \\ \mathrm{~N} & 2.76295 & -0.44714 & 3.00980 \\ \mathrm{C} & 2.71429 & -0.02123 & 1.63320 \\ \mathrm{C} & 2.08037 & 1.16465 & 1.29536 \\ \mathrm{C} & 3.67626 & -0.64064 & 0.76377 \\ \mathrm{~N} & 4.47870 & -1.10351 & 0.06090 \\ \mathrm{H} & 1.66898 & 1.79154 & 2.07658 \\ \mathrm{H} & 2.25361 & 1.61340 & 0.32588 \\ \mathrm{H} & 3.66645 & -0.52477 & 3.46298 \\ \mathrm{H} & 1.78244 & -0.89080 & 4.77970 \\ \mathrm{P} & 0.45773 & -0.86515 & -1.26882 \\ \mathrm{P} & -1.22371 & 1.08529 & 0.68609 \\ \mathrm{H} & -0.66066 & -1.59087 & 0.90698 \\ \mathrm{H} & 1.20959 & -2.03330 & 1.02013 \\ \mathrm{C} & 1.76096 & -0.00463 & -2.23860 \\ \mathrm{C} & 0.47920 & -2.58484 & -1.89615 \\ \mathrm{C} & -1.12709 & 2.84292 & 1.23575 \\ \mathrm{C} & -2.85067 & 0.53246 & 1.34589 \\ \mathrm{H} & 2.74654 & -0.37990 & -1.95260 \\ \mathrm{H} & 0.31730 & -2.60194 & -2.97810 \\ \mathrm{H} & -2.01175 & 3.40205 & 0.91569 \\ \mathrm{H} & -2.78170 & 0.43645 & 2.43291 \\ \mathrm{H} & 1.72740 & 1.07335 & -2.06113 \\ \mathrm{H} & 1.60775 & -0.18908 & -3.30653 \\ \mathrm{H} & 1.44871 & -3.03518 & -1.66935 \\ \mathrm{H} & -0.29797 & -3.16730 & -1.39679 \\ \mathrm{H} & -3.10379 & -0.44899 & 0.93816\end{array}$


Supporting Information for Donoghue et al.: Ligand and Substrate Effects on the Mechanisms of the Rhodium...

$\begin{array}{rrrr}\mathrm{H} & -3.64144 & 1.24696 & 1.09767 \\ \mathrm{H} & -1.06886 & 2.88005 & 2.32726 \\ \mathrm{H} & -0.23672 & 3.32423 & 0.82283 \\ \mathrm{C} & -1.15329 & -0.13894 & -1.85065 \\ \mathrm{H} & -1.13443 & -0.02824 & -2.94072 \\ \mathrm{H} & -1.92927 & -0.87532 & -1.61336 \\ \mathrm{C} & -1.45078 & 1.20353 & -1.16189 \\ \mathrm{H} & -2.46358 & 1.54630 & -1.40032 \\ \mathrm{H} & -0.76134 & 1.97765 & -1.51888\end{array}$

$2 a-D I H Y-t s-A$

SCF Energy = -1371.118938 hartree Total Enthalpy $=-1370.788575$ hartree Negative Frequencies: $-705.62 \mathrm{~cm}^{-1}$

\begin{tabular}{|c|c|c|c|}
\hline $\mathrm{Rh}$ & 0.00000 & 0.00000 & 0.00000 \\
\hline O & 2.22256 & 0.00000 & 0.00000 \\
\hline C & 2.73729 & 1.13324 & 0.00000 \\
\hline $\mathrm{N}$ & 2.01829 & 2.26415 & 0.07717 \\
\hline $\mathrm{C}$ & 0.59528 & 2.21629 & 0.31017 \\
\hline $\mathrm{C}$ & 0.14020 & 1.69600 & 1.55453 \\
\hline $\mathrm{C}$ & -0.20535 & 3.04701 & -0.52798 \\
\hline $\mathrm{N}$ & -0.85027 & 3.71511 & -1.23366 \\
\hline $\mathrm{H}$ & 0.88139 & 1.56874 & 2.33785 \\
\hline $\mathrm{H}$ & -0.84921 & 1.97286 & 1.90029 \\
\hline $\mathrm{H}$ & 2.49376 & 3.15996 & 0.06572 \\
\hline $\mathrm{H}$ & 3.82401 & 1.25669 & -0.06855 \\
\hline $\mathrm{P}$ & -2.25849 & -0.28712 & -0.12328 \\
\hline $\mathrm{P}$ & 0.00619 & -0.31195 & -2.36295 \\
\hline $\mathrm{H}$ & -0.09886 & -1.55860 & 0.11534 \\
\hline $\mathrm{H}$ & -0.09083 & 0.02245 & 1.63288 \\
\hline $\mathrm{C}$ & -3.02555 & -1.37525 & 1.13576 \\
\hline $\mathrm{C}$ & -3.26941 & 1.24842 & -0.08402 \\
\hline $\mathrm{C}$ & 0.82324 & -1.87374 & -2.88009 \\
\hline $\mathrm{C}$ & 0.71582 & 0.97169 & -3.47210 \\
\hline $\mathrm{H}$ & -2.89916 & -0.93058 & 2.12631 \\
\hline $\mathrm{H}$ & -4.31937 & 1.00559 & -0.27650 \\
\hline $\mathrm{H}$ & 0.78151 & -2.00129 & -3.96591 \\
\hline $\mathrm{H}$ & 1.79428 & 1.04119 & -3.30613 \\
\hline $\mathrm{H}$ & 0.26137 & 1.94182 & -3.25504 \\
\hline $\mathrm{H}$ & 0.54056 & 0.71559 & -4.52162 \\
\hline $\mathrm{H}$ & 1.86774 & -1.84934 & -2.55799 \\
\hline $\mathrm{H}$ & 0.34085 & -2.72387 & -2.39202 \\
\hline $\mathrm{H}$ & -2.52964 & -2.34823 & 1.13110 \\
\hline $\mathrm{H}$ & -4.09382 & -1.50214 & 0.93566 \\
\hline $\mathrm{H}$ & -3.20038 & 1.71571 & 0.90233 \\
\hline $\mathrm{H}$ & -2.91673 & 1.96688 & -0.82733 \\
\hline $\mathrm{C}$ & -2.62083 & -1.08146 & -1.76317 \\
\hline $\mathrm{H}$ & -2.38458 & -2.14561 & -1.65286 \\
\hline $\mathrm{H}$ & -3.69214 & -1.00544 & -1.98019 \\
\hline $\mathrm{C}$ & -1.77745 & -0.44678 & -2.87958 \\
\hline $\mathrm{H}$ & -2.12222 & 0.57187 & -3.09060 \\
\hline $\mathrm{H}$ & -1.86397 & -1.01630 & -3.81109 \\
\hline
\end{tabular}

$2 a-D I H Y-t s-C$ 
Supporting Information for Donoghue et al.: Ligand and Substrate Effects on the Mechanisms of the Rhodium...

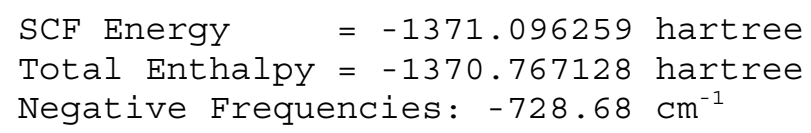

\begin{tabular}{|c|c|c|c|}
\hline $\mathrm{Rh}$ & 0.00000 & 0.00000 & 0.00000 \\
\hline O & 2.24459 & 0.00000 & 0.00000 \\
\hline C & 2.75574 & 1.13074 & 0.00000 \\
\hline $\mathrm{N}$ & 2.04518 & 2.26308 & 0.14782 \\
\hline C & 0.62101 & 2.19661 & 0.45127 \\
\hline $\mathrm{C}$ & 0.25442 & 1.50133 & 1.66124 \\
\hline C & -0.09552 & 3.41663 & 0.11370 \\
\hline $\mathrm{N}$ & -0.69026 & 4.38442 & -0.12717 \\
\hline $\mathrm{H}$ & 1.06404 & 1.19639 & 2.31424 \\
\hline $\mathrm{H}$ & -0.66996 & 1.79001 & 2.14674 \\
\hline $\mathrm{H}$ & 2.50342 & 3.16588 & 0.12281 \\
\hline $\mathrm{H}$ & 3.83540 & 1.26391 & -0.13366 \\
\hline $\mathrm{P}$ & -2.23215 & -0.07883 & -0.38656 \\
\hline $\mathrm{P}$ & -0.26855 & -2.02777 & 1.10520 \\
\hline $\mathrm{H}$ & -0.01878 & -0.77516 & -1.39422 \\
\hline $\mathrm{H}$ & 0.19562 & 1.49371 & -0.77585 \\
\hline C & -3.26230 & 0.95660 & 0.73178 \\
\hline $\mathrm{C}$ & -2.80005 & 0.37475 & -2.06798 \\
\hline C & 0.28967 & -2.20423 & 2.84751 \\
\hline $\mathrm{C}$ & 0.56391 & -3.40399 & 0.22097 \\
\hline $\mathrm{H}$ & -3.00633 & 2.01145 & 0.59795 \\
\hline $\mathrm{H}$ & -3.88307 & 0.24406 & -2.15638 \\
\hline $\mathrm{H}$ & 0.06980 & -3.20685 & 3.22769 \\
\hline $\mathrm{H}$ & 1.63415 & -3.18865 & 0.16078 \\
\hline $\mathrm{H}$ & -3.08474 & 0.68486 & 1.77499 \\
\hline $\mathrm{H}$ & -4.32401 & 0.82340 & 0.50106 \\
\hline $\mathrm{H}$ & -2.55129 & 1.42135 & -2.26375 \\
\hline $\mathrm{H}$ & -2.28872 & -0.24546 & -2.80682 \\
\hline $\mathrm{H}$ & 0.17662 & -3.47500 & -0.79767 \\
\hline $\mathrm{H}$ & 0.41470 & -4.35445 & 0.74238 \\
\hline $\mathrm{H}$ & 1.36930 & -2.03761 & 2.89617 \\
\hline $\mathrm{H}$ & -0.20543 & -1.46043 & 3.47620 \\
\hline $\mathrm{C}$ & -2.78376 & -1.83128 & -0.10937 \\
\hline $\mathrm{H}$ & -3.87419 & -1.87167 & -0.01246 \\
\hline $\mathrm{H}$ & -2.51321 & -2.38737 & -1.01341 \\
\hline $\mathrm{C}$ & -2.08741 & -2.42606 & 1.12527 \\
\hline $\mathrm{H}$ & -2.24046 & -3.50883 & 1.18447 \\
\hline $\mathrm{H}$ & -2.49732 & -1.99260 & 2.0445 \\
\hline
\end{tabular}

$2 a-A L H Y-A$

$\begin{array}{lll}\text { SCF Energy } & =-1371.162041 & \text { hartree } \\ \text { Total Enthalpy } & =-1370.827011 \text { hartree }\end{array}$

$\begin{array}{rrrr}\mathrm{Rh} & 0.78632 & -0.09994 & 0.89966 \\ \mathrm{O} & 0.83846 & -0.25173 & 3.04849 \\ \mathrm{C} & 2.01101 & -0.28018 & 3.48833 \\ \mathrm{~N} & 3.07430 & -0.26723 & 2.68761 \\ \mathrm{C} & 2.91126 & -0.38554 & 1.23487 \\ \mathrm{C} & 4.07229 & 0.25062 & 0.49139 \\ \mathrm{C} & 2.57411 & -1.75373 & 0.87806 \\ \mathrm{~N} & 2.03863 & -2.76005 & 0.60273 \\ \mathrm{H} & 5.03928 & -0.12991 & 0.84947\end{array}$


Supporting Information for Donoghue et al.: Ligand and Substrate Effects on the Mechanisms of the Rhodium...

$\begin{array}{lrrr}\mathrm{H} & 4.01898 & 0.02459 & -0.57551 \\ \mathrm{H} & 4.00174 & -0.33760 & 3.09643 \\ \mathrm{H} & 2.19549 & -0.31557 & 4.56698 \\ \mathrm{P} & 0.66473 & 0.25208 & -1.36234 \\ \mathrm{P} & -1.59163 & -0.10320 & 0.82841 \\ \mathrm{H} & 0.82763 & 1.41957 & 0.99263 \\ \mathrm{H} & 4.06091 & 1.33532 & 0.62211 \\ \mathrm{C} & 1.71301 & 1.55152 & -2.12404 \\ \mathrm{C} & 0.96013 & -1.25344 & -2.37384 \\ \mathrm{C} & -2.37918 & 1.33800 & 1.65377 \\ \mathrm{C} & -2.45697 & -1.56569 & 1.52904 \\ \mathrm{H} & 2.76826 & 1.28242 & -2.04598 \\ \mathrm{H} & 0.81245 & -1.03090 & -3.43518 \\ \mathrm{H} & -3.46932 & 1.28984 & 1.57412 \\ \mathrm{H} & -2.23760 & -1.63070 & 2.59803 \\ \mathrm{H} & 1.55723 & 2.49966 & -1.60405 \\ \mathrm{H} & 1.45804 & 1.67141 & -3.18144 \\ \mathrm{H} & 1.97935 & -1.61347 & -2.21769 \\ \mathrm{H} & 0.27855 & -2.05102 & -2.06927 \\ \mathrm{H} & -2.09529 & -2.47813 & 1.04851 \\ \mathrm{H} & -3.53952 & -1.48593 & 1.39028 \\ \mathrm{H} & -2.09608 & 1.33892 & 2.70983 \\ \mathrm{H} & -2.02289 & 2.26885 & 1.20558 \\ \mathrm{C} & -1.07418 & 0.78398 & -1.76706 \\ \mathrm{H} & -1.13780 & 1.85000 & -1.51919 \\ \mathrm{H} & -1.24597 & 0.69176 & -2.84506 \\ \mathrm{C} & -2.10553 & -0.02042 & -0.96035 \\ \mathrm{H} & -2.15808 & -1.05229 & -1.32603 \\ \mathrm{H} & -3.10841 & 0.40835 & -1.06169\end{array}$

$2 \mathrm{a}-\mathrm{ALHY}-\mathrm{C}$

SCF Energy $\quad=-1371.141627$ hartree Total Enthalpy $=-1370.806211$ hartree

$\begin{array}{rrrr}\mathrm{Rh} & 0.64917 & 0.16429 & 0.78144 \\ \mathrm{O} & 0.63389 & 0.24867 & 2.93650 \\ \mathrm{C} & 1.62631 & 0.36715 & 3.68409 \\ \mathrm{~N} & 2.90201 & 0.18413 & 3.35265 \\ \mathrm{C} & 3.39735 & -0.27571 & 2.03370 \\ \mathrm{C} & 2.73463 & 0.43782 & 0.84136 \\ \mathrm{C} & 4.85614 & -0.06595 & 2.03181 \\ \mathrm{~N} & 6.00335 & 0.10649 & 2.00537 \\ \mathrm{H} & 2.91616 & 1.52007 & 0.92421 \\ \mathrm{H} & 3.24963 & 0.08589 & -0.05516 \\ \mathrm{H} & 3.58700 & 0.29281 & 4.09096 \\ \mathrm{H} & 1.46815 & 0.64908 & 4.73270 \\ \mathrm{P} & -1.76355 & -0.23029 & 0.69474 \\ \mathrm{P} & 0.52743 & 0.26393 & -1.48569 \\ \mathrm{H} & 0.91826 & -1.30517 & 0.55920 \\ \mathrm{H} & 3.22418 & -1.35744 & 1.95922 \\ \mathrm{C} & -2.27722 & -1.95104 & 1.11068 \\ \mathrm{C} & -2.94737 & 0.81436 & 1.64477 \\ \mathrm{C} & 1.77255 & -0.60908 & -2.51228 \\ \mathrm{C} & 0.55190 & 1.99928 & -2.10113 \\ \mathrm{H} & -2.03907 & -2.15212 & 2.15881 \\ \mathrm{H} & -3.98488 & 0.54093 & 1.42949\end{array}$


Supporting Information for Donoghue et al.: Ligand and Substrate Effects on the Mechanisms of the Rhodium...

$\begin{array}{rrrr}\mathrm{H} & 1.52247 & -0.51832 & -3.57354 \\ \mathrm{H} & 1.51800 & 2.45585 & -1.86996 \\ \mathrm{H} & -0.23190 & 2.58765 & -1.61679 \\ \mathrm{H} & 0.39813 & 2.02528 & -3.18458 \\ \mathrm{H} & 2.76250 & -0.17665 & -2.34716 \\ \mathrm{H} & 1.80493 & -1.66646 & -2.23857 \\ \mathrm{H} & -1.72549 & -2.66670 & 0.49577 \\ \mathrm{H} & -3.35089 & -2.09441 & 0.95551 \\ \mathrm{H} & -2.75945 & 0.68120 & 2.71371 \\ \mathrm{H} & -2.79730 & 1.86955 & 1.40057 \\ \mathrm{C} & -1.11044 & -0.43791 & -2.02791 \\ \mathrm{H} & -1.31027 & -0.14811 & -3.06569 \\ \mathrm{H} & -0.99888 & -1.52844 & -2.01537 \\ \mathrm{C} & -2.25202 & -0.00071 & -1.09291 \\ \mathrm{H} & -3.17236 & -0.54861 & -1.32147 \\ \mathrm{H} & -2.47237 & 1.06416 & -1.23139\end{array}$

\section{DuPHOS Ligand, Substrate a, Major Manifold}

\begin{tabular}{|c|c|c|c|}
\hline \multicolumn{2}{|c|}{ SCF Energy } & \multicolumn{2}{|c|}{-1834.475911 hartree } \\
\hline Total & Enthalpy $=$ & 38857 har & \\
\hline $\mathrm{Rh}$ & 0.70611 & -0.01988 & 0.92307 \\
\hline 0 & 0.68253 & 0.01528 & 3.11576 \\
\hline $\mathrm{C}$ & 1.82943 & 0.02992 & 3.60731 \\
\hline $\mathrm{N}$ & 2.93511 & 0.12543 & 2.86351 \\
\hline $\mathrm{C}$ & 2.84215 & 0.30146 & 1.42295 \\
\hline $\mathrm{C}$ & 2.21157 & 1.47783 & 0.92844 \\
\hline $\mathrm{C}$ & 3.78728 & -0.45980 & 0.67090 \\
\hline $\mathrm{N}$ & 4.55042 & -1.07828 & 0.04788 \\
\hline $\mathrm{H}$ & 1.91761 & 2.24596 & 1.63647 \\
\hline $\mathrm{H}$ & 2.46124 & 1.83654 & -0.06224 \\
\hline $\mathrm{H}$ & 3.84264 & 0.14480 & 3.31314 \\
\hline $\mathrm{H}$ & 1.97445 & -0.03005 & 4.69120 \\
\hline $\mathrm{P}$ & -1.60179 & 0.33882 & 0.73925 \\
\hline $\mathrm{P}$ & 0.63520 & -0.34161 & -1.34886 \\
\hline $\mathrm{C}$ & -2.07442 & 0.33987 & -1.03688 \\
\hline $\mathrm{C}$ & -1.06890 & 0.01998 & -1.97525 \\
\hline $\mathrm{C}$ & -2.73424 & -0.83185 & 1.70295 \\
\hline $\mathrm{C}$ & -2.31509 & 1.91013 & 1.51638 \\
\hline $\mathrm{C}$ & 1.01221 & -2.10593 & -1.93611 \\
\hline $\mathrm{C}$ & 1.91336 & 0.47179 & -2.47556 \\
\hline $\mathrm{C}$ & -1.40270 & -0.03879 & -3.34064 \\
\hline $\mathrm{C}$ & -3.37724 & 0.61939 & -1.48303 \\
\hline $\mathrm{C}$ & -3.25583 & -2.05267 & 0.94459 \\
\hline $\mathrm{C}$ & -1.29260 & 2.98385 & 1.87712 \\
\hline $\mathrm{C}$ & 1.08458 & -3.16099 & -0.82706 \\
\hline $\mathrm{C}$ & 1.55315 & 1.84360 & -3.04930 \\
\hline $\mathrm{H}$ & -2.08776 & -1.18213 & 2.51671 \\
\hline $\mathrm{H}$ & -2.99780 & 2.31687 & 0.76137 \\
\hline $\mathrm{H}$ & 0.18093 & -2.36004 & -2.60574 \\
\hline $\mathrm{H}$ & 2.78335 & 0.59376 & -1.81798 \\
\hline $\mathrm{C}$ & -3.81818 & 0.08053 & 2.31598 \\
\hline
\end{tabular}


Supporting Information for Donoghue et al.: Ligand and Substrate Effects on the Mechanisms of the Rhodium...

$\begin{array}{rrrr}\mathrm{C} & -3.14719 & 1.40271 & 2.71341 \\ \mathrm{C} & 2.30992 & -1.97179 & -2.77023 \\ \mathrm{C} & 2.28330 & -0.62201 & -3.50616 \\ \mathrm{H} & -4.61308 & 0.26550 & 1.58006 \\ \mathrm{H} & -4.29002 & -0.41162 & 3.17063 \\ \mathrm{H} & -2.48212 & 1.23080 & 3.56899 \\ \mathrm{H} & -3.87370 & 2.16517 & 3.01884 \\ \mathrm{H} & 3.18025 & -2.00554 & -2.10200 \\ \mathrm{H} & 2.39994 & -2.81694 & -3.46311 \\ \mathrm{H} & 1.54445 & -0.64404 & -4.32039 \\ \mathrm{H} & 3.25079 & -0.39052 & -3.96571 \\ \mathrm{H} & -0.74125 & 3.33559 & 1.00486 \\ \mathrm{H} & -1.80445 & 3.84463 & 2.31615 \\ \mathrm{H} & -0.57143 & 2.60464 & 2.60742 \\ \mathrm{H} & -2.44135 & -2.66775 & 0.55058 \\ \mathrm{H} & -3.85000 & -2.68010 & 1.61694 \\ \mathrm{H} & -3.89279 & -1.76702 & 0.10609 \\ \mathrm{H} & 2.40129 & 2.23604 & -3.61596 \\ \mathrm{H} & 0.69601 & 1.79482 & -3.72259 \\ \mathrm{H} & 1.31997 & 2.56696 & -2.26313 \\ \mathrm{H} & 1.31062 & -4.14010 & -1.26197 \\ \mathrm{H} & 1.87531 & -2.93064 & -0.10854 \\ \mathrm{H} & 0.13766 & -3.25155 & -0.28378 \\ \mathrm{C} & -2.69983 & 0.23782 & -3.76779 \\ \mathrm{C} & -3.68632 & 0.57345 & -2.84072 \\ \mathrm{H} & -0.65311 & -0.29667 & -4.08091 \\ \mathrm{H} & -2.94037 & 0.19395 & -4.82563 \\ \mathrm{H} & -4.69534 & 0.79260 & -3.17442 \\ \mathrm{H} & -4.15579 & 0.88155 & -0.77325\end{array}$

3a-MOLH $-\mathrm{A}$

SCF Energy = $\quad-1835.641390$ hartree

Total Enthalpy $=-1835.088055$ hartree

$\begin{array}{rrrr}\text { Rh } & 0.17430 & 0.97584 & 0.88531 \\ \mathrm{O} & 0.07902 & 0.88813 & 3.06739 \\ \mathrm{C} & 1.21603 & 0.88576 & 3.58366 \\ \mathrm{~N} & 2.32691 & 0.94495 & 2.85177 \\ \mathrm{C} & 2.24722 & 1.20580 & 1.41267 \\ \mathrm{C} & 1.74205 & 2.48785 & 1.02020 \\ \mathrm{C} & 3.23290 & 0.43987 & 0.70515 \\ \mathrm{~N} & 4.04360 & -0.22776 & 0.20360 \\ \mathrm{H} & 1.56260 & 3.21928 & 1.80242 \\ \mathrm{H} & 2.03137 & 2.89970 & 0.06132 \\ \mathrm{H} & 3.23545 & 0.95010 & 3.30350 \\ \mathrm{H} & 1.33813 & 0.82733 & 4.67073 \\ \mathrm{P} & 0.01723 & 0.90270 & -1.44122 \\ \mathrm{P} & -0.55381 & -1.31076 & 0.79288 \\ \mathrm{C} & -0.60438 & -0.76366 & -1.95203 \\ \mathrm{C} & -0.88026 & -1.73436 & -0.96801 \\ \mathrm{H} & -1.64494 & 1.56344 & 0.92412 \\ \mathrm{H} & -1.22494 & 2.24097 & 0.94623 \\ \mathrm{C} & -1.25957 & 2.18573 & -2.00996 \\ \mathrm{C} & 1.28807 & 1.39318 & -2.76478 \\ \mathrm{C} & -2.04233 & -1.93234 & 1.77895 \\ \mathrm{C} & 0.64152 & -2.59468 & 1.50325\end{array}$


Supporting Information for Donoghue et al.: Ligand and Substrate Effects on the Mechanisms of the Rhodium...

\begin{tabular}{|c|c|c|c|}
\hline $\mathrm{H}$ & -1.15122 & 2.98577 & -1.26734 \\
\hline $\mathrm{H}$ & 1.19100 & -2.02725 & 2.26593 \\
\hline $\mathrm{H}$ & -2.61781 & -2.55459 & 1.08244 \\
\hline $\mathrm{H}$ & 1.09244 & 0.66048 & -3.55663 \\
\hline C & -1.36229 & -2.30692 & -3.67346 \\
\hline C & -0.85379 & -1.06448 & -3.30198 \\
\hline $\mathrm{H}$ & -1.55180 & -2.52037 & -4.72102 \\
\hline $\mathrm{H}$ & -0.66622 & -0.32830 & -4.07675 \\
\hline C & -1.63028 & -3.26952 & -2.69972 \\
\hline C & -1.39311 & -2.98207 & -1.35778 \\
\hline $\mathrm{H}$ & -2.02326 & -4.24088 & -2.98444 \\
\hline $\mathrm{H}$ & -1.60638 & -3.74106 & -0.61139 \\
\hline $\mathrm{C}$ & -0.25857 & -3.61734 & 2.22930 \\
\hline $\mathrm{H}$ & 0.31926 & -4.17328 & 2.97615 \\
\hline $\mathrm{H}$ & -0.63541 & -4.35709 & 1.51054 \\
\hline $\mathrm{C}$ & -1.42772 & -2.84988 & 2.86037 \\
\hline $\mathrm{H}$ & -1.06385 & -2.23628 & 3.69530 \\
\hline $\mathrm{H}$ & -2.19065 & -3.52152 & 3.26851 \\
\hline C & 0.80044 & 2.76819 & -3.26806 \\
\hline $\mathrm{H}$ & 1.11860 & 3.55609 & -2.57204 \\
\hline $\mathrm{H}$ & 1.26284 & 2.99474 & -4.23485 \\
\hline $\mathrm{C}$ & -0.72874 & 2.72407 & -3.35466 \\
\hline $\mathrm{H}$ & -1.15896 & 3.70961 & -3.56266 \\
\hline $\mathrm{H}$ & -1.04129 & 2.06383 & -4.17408 \\
\hline $\mathrm{C}$ & 2.78057 & 1.33077 & -2.44844 \\
\hline $\mathrm{H}$ & 3.34207 & 1.59124 & -3.35223 \\
\hline $\mathrm{H}$ & 3.07390 & 2.04264 & -1.67271 \\
\hline $\mathrm{H}$ & 3.10182 & 0.33683 & -2.13498 \\
\hline $\mathrm{C}$ & -2.71738 & 1.72248 & -2.03287 \\
\hline $\mathrm{H}$ & -3.36238 & 2.55635 & -2.32877 \\
\hline $\mathrm{H}$ & -2.87253 & 0.90963 & -2.74779 \\
\hline $\mathrm{H}$ & -3.05859 & 1.37672 & -1.05186 \\
\hline $\mathrm{C}$ & 1.65347 & -3.19451 & 0.52364 \\
\hline $\mathrm{H}$ & 2.30206 & -3.90048 & 1.05360 \\
\hline $\mathrm{H}$ & 1.15910 & -3.74144 & -0.28446 \\
\hline $\mathrm{H}$ & 2.29765 & -2.43071 & 0.07896 \\
\hline C & -2.95271 & -0.83159 & 2.32838 \\
\hline $\mathrm{H}$ & -3.76921 & -1.27575 & 2.90786 \\
\hline $\mathrm{H}$ & -2.40264 & -0.15133 & 2.98524 \\
\hline $\mathrm{H}$ & -3.40794 & -0.24237 & 1.52538 \\
\hline
\end{tabular}

3a-MOLH-C

SCF Energy $=-1835.654677$ hartree

Total Enthalpy $=-1835.101210$ hartree

$\begin{array}{rrrr}\mathrm{Rh} & 0.48170 & -0.30189 & 0.86467 \\ \mathrm{O} & 0.40013 & -0.26223 & 3.06707 \\ \mathrm{C} & 1.54283 & -0.27824 & 3.57765 \\ \mathrm{~N} & 2.65593 & -0.20851 & 2.84622 \\ \mathrm{C} & 2.55902 & 0.05592 & 1.42028 \\ \mathrm{C} & 1.88001 & 1.26251 & 1.01231 \\ \mathrm{C} & 3.57977 & -0.55134 & 0.62677 \\ \mathrm{~N} & 4.39401 & -1.03765 & -0.05065 \\ \mathrm{H} & 1.59577 & 1.96428 & 1.79163 \\ \mathrm{H} & 2.16157 & 1.73137 & 0.07753 \\ \mathrm{H} & 3.56162 & -0.20578 & 3.30235\end{array}$


Supporting Information for Donoghue et al.: Ligand and Substrate Effects on the Mechanisms of the Rhodium...

\begin{tabular}{|c|c|c|c|}
\hline $\mathrm{H}$ & 1.66815 & -0.35360 & 4.66289 \\
\hline $\mathrm{P}$ & 0.52039 & -0.41815 & -1.44380 \\
\hline $\mathrm{P}$ & -1.76857 & 0.39283 & 0.59798 \\
\hline $\mathrm{C}$ & -1.16223 & -0.00806 & -2.09426 \\
\hline $\mathrm{C}$ & -2.16671 & 0.40308 & -1.19564 \\
\hline $\mathrm{H}$ & -0.32238 & -2.12349 & 1.08703 \\
\hline $\mathrm{H}$ & 0.44484 & -2.25175 & 1.10947 \\
\hline $\mathrm{C}$ & 1.76746 & 0.63912 & -2.38413 \\
\hline $\mathrm{C}$ & 1.01896 & -2.03676 & -2.29916 \\
\hline $\mathrm{C}$ & -2.37738 & 2.03468 & 1.30707 \\
\hline $\mathrm{C}$ & -3.05154 & -0.64813 & 1.50982 \\
\hline $\mathrm{H}$ & 2.61954 & 0.70329 & -1.69761 \\
\hline $\mathrm{H}$ & -2.48036 & -1.02980 & 2.36745 \\
\hline $\mathrm{H}$ & 0.21798 & -2.19950 & -3.03107 \\
\hline $\mathrm{H}$ & -2.98004 & 2.47893 & 0.50456 \\
\hline $\mathrm{C}$ & -3.70007 & 0.71565 & -3.05473 \\
\hline $\mathrm{C}$ & -3.42855 & 0.76968 & -1.68956 \\
\hline $\mathrm{H}$ & -4.68120 & 0.99909 & -3.42355 \\
\hline $\mathrm{H}$ & -4.20909 & 1.09383 & -1.00782 \\
\hline $\mathrm{C}$ & -2.71181 & 0.29015 & -3.94372 \\
\hline $\mathrm{C}$ & -1.45229 & -0.06786 & -3.46741 \\
\hline $\mathrm{H}$ & -2.91948 & 0.24113 & -5.00837 \\
\hline $\mathrm{H}$ & -0.69600 & -0.38770 & -4.17674 \\
\hline $\mathrm{C}$ & 2.22310 & -0.24409 & -3.56636 \\
\hline $\mathrm{H}$ & 3.18338 & 0.11570 & -3.95108 \\
\hline $\mathrm{H}$ & 1.50591 & -0.17189 & -4.39396 \\
\hline $\mathrm{C}$ & 2.32257 & -1.68701 & -3.05586 \\
\hline $\mathrm{H}$ & 3.17616 & -1.78013 & -2.37424 \\
\hline $\mathrm{H}$ & 2.47953 & -2.40034 & -3.87207 \\
\hline $\mathrm{C}$ & -3.31825 & 1.62831 & 2.46447 \\
\hline $\mathrm{H}$ & -2.72134 & 1.41854 & 3.36160 \\
\hline $\mathrm{H}$ & -3.99119 & 2.45560 & 2.71398 \\
\hline $\mathrm{C}$ & -4.08385 & 0.36569 & 2.04615 \\
\hline $\mathrm{H}$ & -4.64633 & -0.06613 & 2.88122 \\
\hline $\mathrm{H}$ & -4.81565 & 0.60576 & 1.26340 \\
\hline $\mathrm{C}$ & -1.28190 & 3.02353 & 1.70874 \\
\hline $\mathrm{H}$ & -1.73272 & 3.94597 & 2.09032 \\
\hline $\mathrm{H}$ & -0.65025 & 2.60721 & 2.49954 \\
\hline $\mathrm{H}$ & -0.64305 & 3.29272 & 0.86230 \\
\hline $\mathrm{C}$ & -3.63475 & -1.83536 & 0.74044 \\
\hline $\mathrm{H}$ & -4.31322 & -2.39948 & 1.38886 \\
\hline $\mathrm{H}$ & -4.20253 & -1.51291 & -0.13707 \\
\hline $\mathrm{H}$ & -2.85695 & -2.52779 & 0.40060 \\
\hline $\mathrm{C}$ & 1.31425 & 2.05265 & -2.75689 \\
\hline $\mathrm{H}$ & 2.13341 & 2.58084 & -3.25588 \\
\hline $\mathrm{H}$ & 0.46111 & 2.03867 & -3.44089 \\
\hline $\mathrm{H}$ & 1.02799 & 2.64058 & -1.87861 \\
\hline $\mathrm{C}$ & 1.13797 & -3.28633 & -1.42488 \\
\hline $\mathrm{H}$ & 1.45253 & -4.13143 & -2.04656 \\
\hline $\mathrm{H}$ & 1.89066 & -3.15812 & -0.64093 \\
\hline $\mathrm{H}$ & 0.18650 & -3.56090 & -0.9608 \\
\hline
\end{tabular}

$3 a-M O L H-t s-A$

SCF Energy = $\quad-1835.631199$ hartree Total Enthalpy $=-1835.079609$ hartree Negative Frequencies: $-871.64 \mathrm{~cm}^{-1}$ 
Supporting Information for Donoghue et al.: Ligand and Substrate Effects on the Mechanisms of the Rhodium...

\begin{tabular}{|c|c|c|c|}
\hline $\mathrm{Rh}$ & 0.00000 & 0.00000 & 0.00000 \\
\hline 0 & 2.20180 & 0.00000 & 0.00000 \\
\hline C & 2.71107 & 1.13799 & 0.00000 \\
\hline $\mathrm{N}$ & 1.98102 & 2.25627 & 0.03449 \\
\hline C & 0.54755 & 2.19751 & 0.26218 \\
\hline C & 0.09820 & 1.63398 & 1.48153 \\
\hline C & -0.17812 & 3.16076 & -0.50563 \\
\hline $\mathrm{N}$ & -0.71926 & 3.96797 & -1.14736 \\
\hline $\mathrm{H}$ & 0.83608 & 1.38770 & 2.23714 \\
\hline $\mathrm{H}$ & -0.88954 & 1.87626 & 1.84977 \\
\hline $\mathrm{H}$ & 2.44401 & 3.15880 & 0.03784 \\
\hline $\mathrm{H}$ & 3.79877 & 1.26336 & -0.03918 \\
\hline P & -2.29519 & -0.31091 & -0.13606 \\
\hline $\mathrm{P}$ & -0.00611 & -0.32886 & -2.42048 \\
\hline C & -2.71086 & -0.82569 & -1.86130 \\
\hline C & -1.71555 & -0.84935 & -2.85840 \\
\hline $\mathrm{H}$ & -0.01574 & -1.58635 & 0.23604 \\
\hline $\mathrm{H}$ & -0.02253 & -0.96519 & 1.28705 \\
\hline C & -2.88470 & -1.65132 & 1.06009 \\
\hline C & -3.66977 & 0.90755 & 0.34565 \\
\hline C & 1.14615 & -1.63258 & -3.15857 \\
\hline C & 0.53624 & 1.04221 & -3.61139 \\
\hline $\mathrm{H}$ & -2.17938 & -1.54603 & 1.89294 \\
\hline $\mathrm{H}$ & 1.20070 & 1.65920 & -2.99266 \\
\hline $\mathrm{H}$ & 0.51889 & -2.20120 & -3.85673 \\
\hline $\mathrm{H}$ & -4.41648 & 0.71745 & -0.43555 \\
\hline C & -2.05591 & -1.26348 & -4.15650 \\
\hline $\mathrm{H}$ & -1.30131 & -1.28494 & -4.93674 \\
\hline C & -4.02160 & -1.21387 & -2.18851 \\
\hline $\mathrm{H}$ & -4.80201 & -1.21034 & -1.43429 \\
\hline C & -3.35776 & -1.64789 & -4.46759 \\
\hline $\mathrm{H}$ & -3.60258 & -1.96444 & -5.47700 \\
\hline C & -4.34316 & -1.62341 & -3.48019 \\
\hline $\mathrm{H}$ & -5.35983 & -1.92509 & -3.71332 \\
\hline C & 1.39890 & 0.32750 & -4.67507 \\
\hline $\mathrm{H}$ & 0.75253 & -0.07826 & -5.46463 \\
\hline $\mathrm{H}$ & 2.07436 & 1.04111 & -5.16000 \\
\hline C & 2.16051 & -0.81139 & -3.98467 \\
\hline $\mathrm{H}$ & 2.93066 & -0.40015 & -3.31809 \\
\hline $\mathrm{H}$ & 2.67635 & -1.45758 & -4.70314 \\
\hline C & -4.22536 & 0.34722 & 1.67176 \\
\hline $\mathrm{H}$ & -3.57765 & 0.64816 & 2.50619 \\
\hline $\mathrm{H}$ & -5.21491 & 0.77346 & 1.86927 \\
\hline C & -4.26663 & -1.18226 & 1.55956 \\
\hline $\mathrm{H}$ & -4.50084 & -1.65787 & 2.51794 \\
\hline $\mathrm{H}$ & -5.05182 & -1.48927 & 0.85667 \\
\hline C & -2.82085 & -3.09037 & 0.54624 \\
\hline $\mathrm{H}$ & -1.80688 & -3.37043 & 0.24294 \\
\hline $\mathrm{H}$ & -3.12687 & -3.77684 & 1.34232 \\
\hline $\mathrm{H}$ & -3.48551 & -3.25005 & -0.30721 \\
\hline C & -3.38805 & 2.40815 & 0.35213 \\
\hline $\mathrm{H}$ & -3.00324 & 2.76743 & -0.60342 \\
\hline $\mathrm{H}$ & -4.32246 & 2.94278 & 0.55413 \\
\hline $\mathrm{H}$ & -2.68116 & 2.69705 & 1.13429 \\
\hline C & 1.77431 & -2.60417 & -2.15703 \\
\hline $\mathrm{H}$ & 1.01469 & -3.19343 & -1.63430 \\
\hline
\end{tabular}


Supporting Information for Donoghue et al.: Ligand and Substrate Effects on the Mechanisms of the Rhodium...

$\begin{array}{rrrr}\mathrm{H} & 2.42937 & -3.30634 & -2.68446 \\ \mathrm{H} & 2.37032 & -2.07647 & -1.40797 \\ \mathrm{C} & -0.57016 & 1.94075 & -4.17159 \\ \mathrm{H} & -1.10258 & 2.47588 & -3.38130 \\ \mathrm{H} & -0.12674 & 2.69351 & -4.83252 \\ \mathrm{H} & -1.29759 & 1.37235 & -4.75805\end{array}$

$3 a-M O L H-t s-C$

SCF Energy $=-1835.636429$ hartree
Total Enthalpy $=-1835.084896$ hartree
Negative Frequencies: $-812.93 \mathrm{~cm}^{-1}$

$\begin{array}{rrrr}\mathrm{Rh} & 0.00000 & 0.00000 & 0.00000 \\ \mathrm{O} & 2.20678 & 0.00000 & 0.00000 \\ \mathrm{C} & 2.67439 & 1.15589 & 0.00000 \\ \mathrm{~N} & 1.91098 & 2.24417 & 0.13954 \\ \mathrm{C} & 0.50306 & 2.11799 & 0.47114 \\ \mathrm{C} & 0.15264 & 1.47940 & 1.68367 \\ \mathrm{C} & -0.33395 & 3.09876 & -0.15574 \\ \mathrm{~N} & -1.00859 & 3.91303 & -0.64223 \\ \mathrm{H} & 0.94747 & 1.19609 & 2.36172 \\ \mathrm{H} & -0.81219 & 1.67675 & 2.13213 \\ \mathrm{H} & 2.33348 & 3.16571 & 0.11881 \\ \mathrm{H} & 3.74979 & 1.32578 & -0.12052 \\ \mathrm{P} & -2.28832 & -0.11254 & -0.19365 \\ \mathrm{P} & -0.18179 & -2.05185 & 1.27143 \\ \mathrm{C} & -2.83910 & -1.77249 & 0.40754 \\ \mathrm{C} & -1.92659 & -2.61186 & 1.08021 \\ \mathrm{H} & 0.00956 & -0.95258 & -1.28534 \\ \mathrm{H} & 0.07857 & 0.32118 & -1.56853 \\ \mathrm{C} & -3.30678 & 1.19324 & 0.70882 \\ \mathrm{C} & -3.11425 & 0.15875 & -1.87043 \\ \mathrm{C} & 0.28280 & -2.41266 & 3.07316 \\ \mathrm{C} & 0.97853 & -3.35714 & 0.56041 \\ \mathrm{H} & -2.64862 & 2.06879 & 0.73817 \\ \mathrm{H} & 1.83363 & -2.75285 & 0.22977 \\ \mathrm{H} & -0.52956 & -3.05108 & 3.44256 \\ \mathrm{C} & -3.88942 & 1.48529 & -1.69285 \\ \mathrm{H} & -3.20949 & 2.33254 & -1.84464 \\ \mathrm{H} & -4.67661 & 1.56240 & -2.45018 \\ \mathrm{C} & -4.45763 & 1.52472 & -0.26836 \\ \mathrm{H} & -4.88190 & 2.50476 & -0.02599 \\ \mathrm{H} & -5.26718 & 0.79148 & -0.15647 \\ \mathrm{C} & 1.42792 & -4.20581 & 1.76772 \\ \mathrm{H} & 2.36714 & -4.72289 & 1.54269 \\ \mathrm{H} & 0.68251 & -4.98398 & 1.97914 \\ \mathrm{C} & 1.56790 & -3.26486 & 2.97136 \\ \mathrm{H} & 2.43363 & -2.60475 & 2.82703 \\ \mathrm{H} & 1.73581 & -3.81041 & 3.90628 \\ \mathrm{C} & -4.16923 & -2.19381 & 0.24132 \\ \mathrm{H} & -4.88856 & -1.55985 & -0.26703 \\ \mathrm{C} & -2.37078 & -3.84926 & 1.57330 \\ \mathrm{H} & -1.68133 & -4.50950 & 2.09059 \\ \mathrm{C} & -3.69183 & -4.25650 & 1.40004 \\ \mathrm{H} & -4.01511 & -5.21994 & 1.78257 \\ \mathrm{C} & -4.59355 & -3.42697 & 0.73265 \\ & & & \end{array}$


Supporting Information for Donoghue et al.: Ligand and Substrate Effects on the Mechanisms of the Rhodium...

$\begin{array}{rrrr}\mathrm{H} & -5.62492 & -3.73683 & 0.59553 \\ \mathrm{C} & -3.74711 & 0.84354 & 2.13249 \\ \mathrm{H} & -2.89772 & 0.63048 & 2.78993 \\ \mathrm{H} & -4.29427 & 1.68831 & 2.56373 \\ \mathrm{H} & -4.40886 & -0.02704 & 2.15059 \\ \mathrm{C} & 0.40891 & -1.20239 & 3.99882 \\ \mathrm{H} & -0.50481 & -0.60075 & 4.02065 \\ \mathrm{H} & 0.60812 & -1.53618 & 5.02293 \\ \mathrm{H} & 1.24059 & -0.55816 & 3.69783 \\ \mathrm{C} & 0.44924 & -4.14787 & -0.63673 \\ \mathrm{H} & -0.42037 & -4.75622 & -0.37162 \\ \mathrm{H} & 0.16513 & -3.48950 & -1.46386 \\ \mathrm{H} & 1.22879 & -4.82173 & -1.00691 \\ \mathrm{C} & -2.22417 & 0.11477 & -3.11250 \\ \mathrm{H} & -2.84495 & 0.25237 & -4.00437 \\ \mathrm{H} & -1.47673 & 0.91340 & -3.10069 \\ \mathrm{H} & -1.70457 & -0.84211 & -3.21587 \\ \mathrm{H} & -3.83847 & -0.66199 & -1.94050\end{array}$

3a-DIHY-A

SCF Energy $\quad=-1835.637953$ hartree Total Enthalpy $=-1835.083753$ hartree

$\begin{array}{rrrr}\text { Rh } & -0.01715 & 0.79112 & 0.71981 \\ \mathrm{O} & -0.52817 & 0.62050 & 2.88030 \\ \mathrm{C} & 0.43030 & 0.50967 & 3.66135 \\ \mathrm{~N} & 1.71312 & 0.58417 & 3.26854 \\ \mathrm{C} & 2.06525 & 0.97095 & 1.92481 \\ \mathrm{C} & 1.65729 & 2.21160 & 1.44596 \\ \mathrm{C} & 3.12588 & 0.20946 & 1.34275 \\ \mathrm{~N} & 4.01936 & -0.40682 & 0.92164 \\ \mathrm{H} & 1.18199 & 2.90835 & 2.12516 \\ \mathrm{H} & 2.11130 & 2.62631 & 0.55749 \\ \mathrm{H} & 2.45180 & 0.46903 & 3.95352 \\ \mathrm{H} & 0.26853 & 0.33039 & 4.73067 \\ \mathrm{P} & 0.10223 & 0.92001 & -1.57198 \\ \mathrm{P} & -0.13064 & -1.64544 & 0.42028 \\ \mathrm{C} & -0.26428 & -0.75828 & -2.25008 \\ \mathrm{C} & -0.37662 & -1.87116 & -1.39190 \\ \mathrm{H} & -1.51112 & 0.70488 & 0.31402 \\ \mathrm{H} & -0.51461 & 2.29134 & 0.66789 \\ \mathrm{C} & -1.10158 & 2.17293 & -2.30705 \\ \mathrm{C} & 1.57628 & 1.52722 & -2.60080 \\ \mathrm{C} & -1.55864 & -2.56619 & 1.24825 \\ \mathrm{C} & 1.19532 & -2.86927 & 1.01051 \\ \mathrm{H} & -1.15246 & 2.92715 & -1.51354 \\ \mathrm{H} & 1.70528 & -2.31895 & 1.81089 \\ \mathrm{H} & -1.96194 & -3.21935 & 0.46409 \\ \mathrm{H} & 1.57183 & 0.81835 & -3.43865 \\ \mathrm{C} & -0.72780 & -2.19115 & -4.16240 \\ \mathrm{C} & -0.43749 & -0.93617 & -3.63342 \\ \mathrm{H} & -0.86558 & -2.30745 & -5.23309 \\ \mathrm{H} & -0.35613 & -0.09147 & -4.30999 \\ \mathrm{C} & -0.84357 & -3.29214 & -3.31384 \\ \mathrm{C} & -0.66725 & -3.13063 & -1.94191 \\ \mathrm{H} & -1.06886 & -4.27402 & -3.71895\end{array}$


Supporting Information for Donoghue et al.: Ligand and Substrate Effects on the Mechanisms of the Rhodium...

$\begin{array}{rrrr}\mathrm{H} & -0.75529 & -3.99933 & -1.29678 \\ \mathrm{C} & 0.40682 & -4.03358 & 1.65209 \\ \mathrm{H} & 1.03505 & -4.56417 & 2.37608 \\ \mathrm{H} & 0.13338 & -4.76421 & 0.87932 \\ \mathrm{C} & -0.86199 & -3.46027 & 2.29590 \\ \mathrm{H} & -0.60195 & -2.86248 & 3.18038 \\ \mathrm{H} & -1.54306 & -4.24763 & 2.63651 \\ \mathrm{C} & 1.12399 & 2.90028 & -3.14143 \\ \mathrm{H} & 1.27188 & 3.67082 & -2.37291 \\ \mathrm{H} & 1.74170 & 3.18591 & -3.99975 \\ \mathrm{C} & -0.36371 & 2.80135 & -3.50769 \\ \mathrm{H} & -0.79261 & 3.78163 & -3.74105 \\ \mathrm{H} & -0.49244 & 2.18214 & -4.40483 \\ \mathrm{C} & 2.97373 & 1.50621 & -1.98442 \\ \mathrm{H} & 3.71243 & 1.74032 & -2.75824 \\ \mathrm{H} & 3.08975 & 2.26012 & -1.20056 \\ \mathrm{H} & 3.23460 & 0.53200 & -1.56553 \\ \mathrm{C} & -2.51928 & 1.67165 & -2.58429 \\ \mathrm{H} & -3.13698 & 2.50226 & -2.94051 \\ \mathrm{H} & -2.53827 & 0.88933 & -3.34782 \\ \mathrm{H} & -2.99130 & 1.27618 & -1.67936 \\ \mathrm{C} & 2.24584 & -3.29666 & -0.01867 \\ \mathrm{H} & 2.95488 & -3.98560 & 0.45307 \\ \mathrm{H} & 1.79571 & -3.81501 & -0.86989 \\ \mathrm{H} & 2.82098 & -2.44669 & -0.39317 \\ \mathrm{C} & -2.69507 & -1.69850 & 1.79306 \\ \mathrm{H} & -3.45492 & -2.33672 & 2.25755 \\ \mathrm{H} & -2.33730 & -0.98914 & 2.54305 \\ \mathrm{H} & -3.18625 & -1.12997 & 0.99772\end{array}$

3a-DIHY-C

SCF Energy $=-1835.640833$ hartree Total Enthalpy $=-1835.086883$ hartree

$\begin{array}{rrrr}\mathrm{Rh} & 0.24254 & -0.55591 & 0.76351 \\ \mathrm{O} & 0.06504 & -0.58189 & 2.98069 \\ \mathrm{C} & 1.15565 & -0.70744 & 3.55797 \\ \mathrm{~N} & 2.33899 & -0.62645 & 2.92482 \\ \mathrm{C} & 2.43200 & -0.24419 & 1.53531 \\ \mathrm{C} & 1.99267 & 1.00651 & 1.13074 \\ \mathrm{C} & 3.36241 & -1.01781 & 0.75970 \\ \mathrm{~N} & 4.15015 & -1.61111 & 0.14309 \\ \mathrm{H} & 1.62182 & 1.70326 & 1.86936 \\ \mathrm{H} & 2.27189 & 1.39950 & 0.16228 \\ \mathrm{H} & 3.19618 & -0.78356 & 3.44261 \\ \mathrm{H} & 1.20731 & -0.89691 & 4.63644 \\ \mathrm{P} & 0.25651 & -0.82421 & -1.50093 \\ \mathrm{P} & -1.46570 & 1.16571 & 0.39709 \\ \mathrm{C} & -1.19986 & 0.07611 & -2.19222 \\ \mathrm{C} & -1.94648 & 0.95024 & -1.37306 \\ \mathrm{H} & -1.03286 & -1.43021 & 0.61556 \\ \mathrm{H} & 0.76560 & -2.04464 & 0.85268 \\ \mathrm{C} & 1.79637 & -0.24278 & -2.42532 \\ \mathrm{C} & 0.25034 & -2.55264 & -2.24751 \\ \mathrm{C} & -1.52806 & 3.04047 & 0.69358 \\ \mathrm{C} & -2.95893 & 0.80453 & 1.49549\end{array}$


Supporting Information for Donoghue et al.: Ligand and Substrate Effects on the Mechanisms of the Rhodium...

\begin{tabular}{|c|c|c|c|}
\hline $\mathrm{H}$ & 2.59674 & -0.31437 & -1.68117 \\
\hline $\mathrm{H}$ & -2.48748 & 0.42974 & 2.41346 \\
\hline $\mathrm{H}$ & -0.47133 & -2.48869 & -3.07113 \\
\hline $\mathrm{H}$ & -2.05057 & 3.43725 & -0.18537 \\
\hline $\mathrm{C}$ & -3.38980 & 1.46653 & -3.26614 \\
\hline $\mathrm{C}$ & -3.03749 & 1.63782 & -1.92936 \\
\hline $\mathrm{H}$ & -4.24230 & 2.00146 & -3.67362 \\
\hline $\mathrm{H}$ & -3.62949 & 2.30914 & -1.31503 \\
\hline $\mathrm{C}$ & -2.64623 & 0.60818 & -4.07561 \\
\hline $\mathrm{C}$ & -1.55929 & -0.07976 & -3.54136 \\
\hline $\mathrm{H}$ & -2.91000 & 0.47373 & -5.12018 \\
\hline $\mathrm{H}$ & -0.98910 & -0.73737 & -4.18986 \\
\hline $\mathrm{C}$ & 2.05021 & -1.34552 & -3.47900 \\
\hline $\mathrm{H}$ & 3.09774 & -1.32612 & -3.79710 \\
\hline $\mathrm{H}$ & 1.44105 & -1.15408 & -4.37257 \\
\hline $\mathrm{C}$ & 1.66640 & -2.69277 & -2.85161 \\
\hline $\mathrm{H}$ & 2.38233 & -2.95286 & -2.06169 \\
\hline $\mathrm{H}$ & 1.68425 & -3.50672 & -3.58378 \\
\hline $\mathrm{C}$ & -2.45128 & 3.20146 & 1.92132 \\
\hline $\mathrm{H}$ & -1.87830 & 3.01411 & 2.83950 \\
\hline $\mathrm{H}$ & -2.82364 & 4.22988 & 1.98387 \\
\hline $\mathrm{C}$ & -3.58892 & 2.17957 & 1.80124 \\
\hline $\mathrm{H}$ & -4.19094 & 2.12913 & 2.71507 \\
\hline $\mathrm{H}$ & -4.27143 & 2.46797 & 0.99078 \\
\hline $\mathrm{C}$ & -0.18738 & 3.76474 & 0.82050 \\
\hline $\mathrm{H}$ & -0.35337 & 4.84528 & 0.88973 \\
\hline $\mathrm{H}$ & 0.34716 & 3.46275 & 1.72644 \\
\hline $\mathrm{H}$ & 0.46290 & 3.58905 & -0.04220 \\
\hline $\mathrm{C}$ & -3.93527 & -0.25664 & 0.98449 \\
\hline $\mathrm{H}$ & -4.72150 & -0.42111 & 1.72871 \\
\hline $\mathrm{H}$ & -4.41749 & 0.04843 & 0.05121 \\
\hline $\mathrm{H}$ & -3.43903 & -1.21697 & 0.81409 \\
\hline $\mathrm{C}$ & 1.75399 & 1.18348 & -2.98049 \\
\hline $\mathrm{H}$ & 2.71284 & 1.42193 & -3.45242 \\
\hline $\mathrm{H}$ & 0.97154 & 1.30349 & -3.73495 \\
\hline $\mathrm{H}$ & 1.57777 & 1.92914 & -2.19769 \\
\hline C & -0.17006 & -3.70145 & -1.33111 \\
\hline $\mathrm{H}$ & -0.16409 & -4.63809 & -1.89845 \\
\hline $\mathrm{H}$ & 0.51592 & -3.81238 & -0.48684 \\
\hline $\mathrm{H}$ & -1.17800 & -3.55924 & -0.93078 \\
\hline
\end{tabular}

$3 a-D I H Y-t s-A$

SCF Energy $=-1835.633348$ hartree Total Enthalpy $=-1835.080834$ hartree Negative Frequencies: $-659.50 \mathrm{~cm}^{-1}$

$\begin{array}{rrrr}\mathrm{Rh} & 0.00000 & 0.00000 & 0.00000 \\ \mathrm{O} & 2.22590 & 0.00000 & 0.00000 \\ \mathrm{C} & 2.76295 & 1.12323 & 0.00000 \\ \mathrm{~N} & 2.06593 & 2.26342 & 0.09557 \\ \mathrm{C} & 0.64524 & 2.24050 & 0.34974 \\ \mathrm{C} & 0.20357 & 1.67988 & 1.57761 \\ \mathrm{C} & -0.10967 & 3.17387 & -0.41780 \\ \mathrm{~N} & -0.67969 & 3.96258 & -1.05981 \\ \mathrm{H} & 0.95674 & 1.50585 & 2.33999 \\ \mathrm{H} & -0.77521 & 1.95489 & 1.95014\end{array}$


Supporting Information for Donoghue et al.: Ligand and Substrate Effects on the Mechanisms of the Rhodium...

\begin{tabular}{|c|c|c|c|}
\hline $\mathrm{H}$ & 2.55299 & 3.15254 & 0.07026 \\
\hline $\mathrm{H}$ & 3.85074 & 1.22641 & -0.08361 \\
\hline $\mathrm{P}$ & -2.25218 & -0.45894 & -0.02031 \\
\hline $\mathrm{P}$ & -0.05365 & -0.34856 & -2.36196 \\
\hline $\mathrm{C}$ & -2.68519 & -1.06623 & -1.70594 \\
\hline $\mathrm{C}$ & -1.72486 & -1.02042 & -2.73596 \\
\hline $\mathrm{H}$ & -0.04758 & -1.55604 & 0.09827 \\
\hline $\mathrm{H}$ & -0.04391 & -0.01209 & 1.62619 \\
\hline $\mathrm{C}$ & -2.74900 & -1.73574 & 1.27475 \\
\hline $\mathrm{C}$ & -3.64717 & 0.74404 & 0.42953 \\
\hline $\mathrm{C}$ & 1.18284 & -1.53669 & -3.14751 \\
\hline $\mathrm{C}$ & 0.32324 & 1.08043 & -3.54319 \\
\hline $\mathrm{H}$ & -2.01575 & -1.54863 & 2.06865 \\
\hline $\mathrm{H}$ & 0.94186 & 1.75127 & -2.93362 \\
\hline $\mathrm{H}$ & 0.58352 & -2.13765 & -3.84330 \\
\hline $\mathrm{H}$ & -4.41374 & 0.49687 & -0.31620 \\
\hline $\mathrm{C}$ & -2.07005 & -1.48210 & -4.01618 \\
\hline $\mathrm{H}$ & -1.34458 & -1.44854 & -4.82321 \\
\hline $\mathrm{C}$ & -3.96723 & -1.57013 & -1.98022 \\
\hline $\mathrm{H}$ & -4.71922 & -1.61545 & -1.19864 \\
\hline $\mathrm{C}$ & -3.34318 & -1.98470 & -4.27459 \\
\hline $\mathrm{H}$ & -3.59328 & -2.33869 & -5.27010 \\
\hline $\mathrm{C}$ & -4.29324 & -2.03034 & -3.25385 \\
\hline $\mathrm{H}$ & -5.28618 & -2.42446 & -3.44781 \\
\hline $\mathrm{C}$ & 1.21927 & 0.45877 & -4.63774 \\
\hline $\mathrm{H}$ & 0.59160 & 0.00460 & -5.41601 \\
\hline $\mathrm{H}$ & 1.81406 & 1.23665 & -5.12910 \\
\hline $\mathrm{C}$ & 2.09897 & -0.61535 & -3.98278 \\
\hline $\mathrm{H}$ & 2.84507 & -0.14506 & -3.32804 \\
\hline $\mathrm{H}$ & 2.65274 & -1.20217 & -4.72344 \\
\hline $\mathrm{C}$ & -4.14825 & 0.24926 & 1.80298 \\
\hline $\mathrm{H}$ & -3.49095 & 0.62612 & 2.59840 \\
\hline $\mathrm{H}$ & -5.14788 & 0.64978 & 2.00325 \\
\hline $\mathrm{C}$ & -4.13198 & -1.28581 & 1.78980 \\
\hline $\mathrm{H}$ & -4.31974 & -1.70579 & 2.78368 \\
\hline $\mathrm{H}$ & -4.92575 & -1.66608 & 1.13365 \\
\hline $\mathrm{C}$ & -2.63892 & -3.20236 & 0.85679 \\
\hline $\mathrm{H}$ & -1.62304 & -3.45502 & 0.53716 \\
\hline $\mathrm{H}$ & -2.88536 & -3.84483 & 1.70821 \\
\hline $\mathrm{H}$ & -3.32424 & -3.44969 & 0.04141 \\
\hline $\mathrm{C}$ & -3.37761 & 2.24447 & 0.34328 \\
\hline $\mathrm{H}$ & -3.00471 & 2.54907 & -0.63658 \\
\hline $\mathrm{H}$ & -4.30923 & 2.79018 & 0.52696 \\
\hline $\mathrm{H}$ & -2.65850 & 2.57916 & 1.09606 \\
\hline $\mathrm{C}$ & 1.91281 & -2.48035 & -2.18891 \\
\hline $\mathrm{H}$ & 1.21582 & -3.13854 & -1.66175 \\
\hline $\mathrm{H}$ & 2.60307 & -3.11589 & -2.75448 \\
\hline $\mathrm{H}$ & 2.48689 & -1.92771 & -1.44190 \\
\hline $\mathrm{C}$ & -0.87946 & 1.87523 & -4.05902 \\
\hline $\mathrm{H}$ & -1.42929 & 2.35437 & -3.24501 \\
\hline $\mathrm{H}$ & -0.53016 & 2.67075 & -4.72591 \\
\hline $\mathrm{H}$ & -1.57153 & 1.24558 & -4.6255 \\
\hline
\end{tabular}

$3 a-D I H Y-t s-C$

SCF Energy $\quad=-1835.616580$ hartree

Total Enthalpy $=-1835.065416$ hartree 
Supporting Information for Donoghue et al.: Ligand and Substrate Effects on the Mechanisms of the Rhodium...

Negative Frequencies: $-718.52 \mathrm{~cm}^{-1}$

\begin{tabular}{|c|c|c|c|}
\hline $\mathrm{Rh}$ & 0.00000 & 0.00000 & 0.00000 \\
\hline 0 & 2.26041 & 0.00000 & 0.00000 \\
\hline $\mathrm{C}$ & 2.76830 & 1.13200 & 0.00000 \\
\hline $\mathrm{N}$ & 2.05512 & 2.26130 & 0.15422 \\
\hline $\mathrm{C}$ & 0.63161 & 2.18836 & 0.46570 \\
\hline C & 0.27539 & 1.49551 & 1.67786 \\
\hline C & -0.08568 & 3.40921 & 0.13590 \\
\hline $\mathrm{N}$ & -0.68074 & 4.37918 & -0.09568 \\
\hline $\mathrm{H}$ & 1.09108 & 1.18399 & 2.31825 \\
\hline $\mathrm{H}$ & -0.64548 & 1.77431 & 2.17468 \\
\hline $\mathrm{H}$ & 2.51299 & 3.16445 & 0.14585 \\
\hline $\mathrm{H}$ & 3.84695 & 1.27046 & -0.13877 \\
\hline $\mathrm{P}$ & -2.23159 & -0.05756 & -0.40752 \\
\hline P & -0.24963 & -2.10809 & 0.94371 \\
\hline C & -2.82495 & -1.77147 & -0.08633 \\
\hline C & -1.94420 & -2.68426 & 0.52717 \\
\hline $\mathrm{H}$ & -0.01128 & -0.75752 & -1.39965 \\
\hline $\mathrm{H}$ & 0.20810 & 1.49095 & -0.76668 \\
\hline C & -3.29107 & 1.13867 & 0.59105 \\
\hline C & -2.92072 & 0.46897 & -2.08309 \\
\hline C & 0.04315 & -2.59504 & 2.74310 \\
\hline C & 1.05362 & -3.27141 & 0.24617 \\
\hline $\mathrm{H}$ & -2.62437 & 1.99314 & 0.76580 \\
\hline $\mathrm{H}$ & 1.87974 & -2.58484 & 0.02097 \\
\hline $\mathrm{H}$ & -0.72758 & -3.34997 & 2.94725 \\
\hline C & -3.72623 & 1.75419 & -1.77583 \\
\hline $\mathrm{H}$ & -3.04979 & 2.61869 & -1.76843 \\
\hline $\mathrm{H}$ & -4.46329 & 1.93395 & -2.56532 \\
\hline C & -4.38357 & 1.60065 & -0.39721 \\
\hline $\mathrm{H}$ & -4.83334 & 2.53795 & -0.05250 \\
\hline $\mathrm{H}$ & -5.19102 & 0.85761 & -0.43666 \\
\hline C & 1.47352 & -4.16049 & 1.43725 \\
\hline $\mathrm{H}$ & 2.47328 & -4.57480 & 1.26851 \\
\hline $\mathrm{H}$ & 0.78803 & -5.01384 & 1.52757 \\
\hline C & 1.41497 & -3.30374 & 2.71083 \\
\hline $\mathrm{H}$ & 2.21725 & -2.55390 & 2.69475 \\
\hline $\mathrm{H}$ & 1.55502 & -3.90200 & 3.61752 \\
\hline C & -4.12701 & -2.18136 & -0.41114 \\
\hline $\mathrm{H}$ & -4.82283 & -1.49157 & -0.87833 \\
\hline C & -2.38358 & -3.98705 & 0.80485 \\
\hline $\mathrm{H}$ & -1.71526 & -4.70186 & 1.27595 \\
\hline C & -3.67701 & -4.38501 & 0.47178 \\
\hline $\mathrm{H}$ & -4.00240 & -5.39892 & 0.68399 \\
\hline C & -4.54918 & -3.48101 & -0.13565 \\
\hline $\mathrm{H}$ & -5.55836 & -3.78648 & -0.39477 \\
\hline C & -3.79964 & 0.62197 & 1.93808 \\
\hline $\mathrm{H}$ & -2.97807 & 0.33277 & 2.60132 \\
\hline $\mathrm{H}$ & -4.37097 & 1.40769 & 2.44300 \\
\hline $\mathrm{H}$ & -4.45689 & -0.24398 & 1.81722 \\
\hline C & -0.08140 & -1.47839 & 3.77823 \\
\hline $\mathrm{H}$ & -1.06899 & -1.00755 & 3.75539 \\
\hline $\mathrm{H}$ & 0.06691 & -1.88475 & 4.78431 \\
\hline $\mathrm{H}$ & 0.66990 & -0.70072 & 3.61748 \\
\hline C & 0.67874 & -4.01544 & -1.03713 \\
\hline $\mathrm{H}$ & -0.15855 & -4.70264 & -0.88385 \\
\hline
\end{tabular}


Supporting Information for Donoghue et al.: Ligand and Substrate Effects on the Mechanisms of the Rhodium...

$\begin{array}{rrrr}\mathrm{H} & 0.40922 & -3.32064 & -1.83824 \\ \mathrm{H} & 1.53472 & -4.60427 & -1.38331 \\ \mathrm{C} & -1.91085 & 0.61786 & -3.22176 \\ \mathrm{H} & -2.43585 & 0.91480 & -4.13610 \\ \mathrm{H} & -1.16373 & 1.38506 & -2.99751 \\ \mathrm{H} & -1.38070 & -0.31633 & -3.42479 \\ \mathrm{H} & -3.62562 & -0.32922 & -2.34675\end{array}$

$3 a-A L H Y-A$

SCF Energy $\quad=-1835.679198$ hartree

Total Enthalpy $=-1835.121946$ hartree

\begin{tabular}{|c|c|c|c|}
\hline $\mathrm{Rh}$ & 0.76320 & -0.07242 & 0.87108 \\
\hline 0 & 0.78906 & 0.11003 & 3.03316 \\
\hline C & 1.95276 & 0.16550 & 3.49295 \\
\hline $\mathrm{N}$ & 3.02982 & 0.03287 & 2.72293 \\
\hline C & 2.88466 & -0.37203 & 1.32059 \\
\hline C & 4.09512 & 0.02974 & 0.50026 \\
\hline C & 2.45245 & -1.75570 & 1.25470 \\
\hline $\mathrm{N}$ & 1.81931 & -2.74335 & 1.21431 \\
\hline $\mathrm{H}$ & 5.03006 & -0.34738 & 0.93887 \\
\hline $\mathrm{H}$ & 4.02981 & -0.37549 & -0.51102 \\
\hline $\mathrm{H}$ & 3.94849 & 0.02578 & 3.15629 \\
\hline $\mathrm{H}$ & 2.11744 & 0.32851 & 4.56303 \\
\hline $\mathrm{P}$ & 0.68359 & 0.09347 & -1.43046 \\
\hline $\mathrm{P}$ & -1.59473 & -0.03085 & 0.78403 \\
\hline C & -1.07293 & 0.40183 & -1.92032 \\
\hline C & -2.08728 & 0.34609 & -0.94353 \\
\hline $\mathrm{H}$ & 0.82360 & 1.44613 & 0.76379 \\
\hline $\mathrm{H}$ & 4.16586 & 1.11880 & 0.43650 \\
\hline C & 1.76260 & 1.46482 & -2.13618 \\
\hline C & 1.27941 & -1.21817 & -2.66428 \\
\hline C & -2.57070 & 1.12417 & 1.90711 \\
\hline C & -2.46101 & -1.60345 & 1.36656 \\
\hline $\mathrm{H}$ & 0.47208 & -1.22922 & -3.40698 \\
\hline $\mathrm{H}$ & -3.35557 & 1.54433 & 1.26530 \\
\hline $\mathrm{H}$ & 2.60288 & 1.48877 & -1.43238 \\
\hline $\mathrm{H}$ & -1.73544 & -2.04155 & 2.06399 \\
\hline C & -2.74717 & 0.89658 & -3.61293 \\
\hline C & -1.41978 & 0.67323 & -3.25413 \\
\hline $\mathrm{H}$ & -2.99731 & 1.11373 & -4.64693 \\
\hline $\mathrm{H}$ & -0.65542 & 0.71945 & -4.02323 \\
\hline C & -3.42184 & 0.56573 & -1.31970 \\
\hline C & -3.75063 & 0.84130 & -2.64467 \\
\hline $\mathrm{H}$ & -4.21375 & 0.51870 & -0.57824 \\
\hline $\mathrm{H}$ & -4.78683 & 1.01034 & -2.92157 \\
\hline C & 2.52605 & -0.58849 & -3.32484 \\
\hline $\mathrm{H}$ & 2.72482 & -1.07447 & -4.28616 \\
\hline $\mathrm{H}$ & 3.40802 & -0.76760 & -2.69636 \\
\hline C & 2.28812 & 0.92080 & -3.48287 \\
\hline $\mathrm{H}$ & 1.55449 & 1.10719 & -4.27748 \\
\hline $\mathrm{H}$ & 3.20246 & 1.44900 & -3.77427 \\
\hline C & -3.67004 & -1.09877 & 2.18457 \\
\hline $\mathrm{H}$ & -4.50835 & -0.87611 & 1.51066 \\
\hline $\mathrm{H}$ & -4.01592 & -1.87834 & 2.87204 \\
\hline C & -3.23643 & 0.17284 & 2.92793 \\
\hline
\end{tabular}


Supporting Information for Donoghue et al.: Ligand and Substrate Effects on the Mechanisms of the Rhodium...

$\begin{array}{rrrr}\mathrm{H} & -4.08026 & 0.67263 & 3.41555 \\ \mathrm{H} & -2.51513 & -0.08251 & 3.71487 \\ \mathrm{C} & 1.47717 & -2.64529 & -2.15263 \\ \mathrm{H} & 1.73142 & -3.29836 & -2.99457 \\ \mathrm{H} & 2.28377 & -2.71291 & -1.42047 \\ \mathrm{H} & 0.57275 & -3.04521 & -1.68604 \\ \mathrm{C} & 1.13727 & 2.86054 & -2.18261 \\ \mathrm{H} & 1.87356 & 3.58241 & -2.55071 \\ \mathrm{H} & 0.27020 & 2.89935 & -2.84760 \\ \mathrm{H} & 0.81751 & 3.19321 & -1.18979 \\ \mathrm{C} & -2.78246 & -2.63531 & 0.28402 \\ \mathrm{H} & -3.25125 & -3.51329 & 0.74008 \\ \mathrm{H} & -3.47386 & -2.23799 & -0.46498 \\ \mathrm{H} & -1.87972 & -2.97822 & -0.23123 \\ \mathrm{C} & -1.77229 & 2.27412 & 2.52698 \\ \mathrm{H} & -2.42834 & 2.87691 & 3.16421 \\ \mathrm{H} & -0.94969 & 1.89827 & 3.14006 \\ \mathrm{H} & -1.35555 & 2.93654 & 1.76182\end{array}$

$3 a-A L H Y-C$

SCF Energy $=-1835.663385$ hartree Total Enthalpy $=-1835.105923$ hartree

\begin{tabular}{|c|c|c|c|}
\hline $\mathrm{Rh}$ & 0.59681 & 0.15262 & 0.75167 \\
\hline 0 & 0.53961 & 0.15630 & 2.91961 \\
\hline $\mathrm{C}$ & 1.51628 & 0.25550 & 3.68888 \\
\hline $\mathrm{N}$ & 2.80386 & 0.14053 & 3.37044 \\
\hline $\mathrm{C}$ & 3.34975 & -0.19153 & 2.03274 \\
\hline $\mathrm{C}$ & 2.66155 & 0.56317 & 0.88196 \\
\hline $\mathrm{C}$ & 4.79206 & 0.10812 & 2.08142 \\
\hline $\mathrm{N}$ & 5.92629 & 0.35266 & 2.09470 \\
\hline $\mathrm{H}$ & 2.75655 & 1.64593 & 1.05858 \\
\hline $\mathrm{H}$ & 3.22502 & 0.33339 & -0.02480 \\
\hline $\mathrm{H}$ & 3.46864 & 0.21939 & 4.13046 \\
\hline $\mathrm{H}$ & 1.33433 & 0.45787 & 4.75224 \\
\hline $\mathrm{P}$ & -1.76448 & -0.34604 & 0.62869 \\
\hline $\mathrm{P}$ & 0.52990 & 0.29889 & -1.51341 \\
\hline $\mathrm{C}$ & -2.20247 & -0.34244 & -1.16093 \\
\hline $\mathrm{C}$ & -1.18324 & -0.07303 & -2.10106 \\
\hline $\mathrm{H}$ & 0.93477 & -1.29684 & 0.50192 \\
\hline $\mathrm{H}$ & 3.24826 & -1.27388 & 1.87765 \\
\hline $\mathrm{C}$ & -2.32459 & -1.96712 & 1.41322 \\
\hline $\mathrm{C}$ & -3.06580 & 0.70645 & 1.50215 \\
\hline $\mathrm{C}$ & 1.69118 & -0.67450 & -2.63169 \\
\hline $\mathrm{C}$ & 1.05693 & 1.99202 & -2.15944 \\
\hline $\mathrm{H}$ & -3.85925 & 0.87848 & 0.76427 \\
\hline $\mathrm{H}$ & 1.05523 & -0.99257 & -3.46693 \\
\hline $\mathrm{H}$ & 1.73708 & 2.35682 & -1.37882 \\
\hline $\mathrm{H}$ & -1.59192 & -2.10466 & 2.21976 \\
\hline $\mathrm{C}$ & -2.79109 & -0.32944 & -3.90788 \\
\hline $\mathrm{C}$ & -1.49361 & -0.07174 & -3.47109 \\
\hline $\mathrm{H}$ & -3.01424 & -0.32304 & -4.97048 \\
\hline $\mathrm{H}$ & -0.72537 & 0.13784 & -4.20921 \\
\hline $\mathrm{C}$ & -3.50582 & -0.59455 & -1.61819 \\
\hline $\mathrm{C}$ & -3.79976 & -0.59083 & -2.97978 \\
\hline $\mathrm{H}$ & -4.30089 & -0.79963 & -0.90761 \\
\hline
\end{tabular}


Supporting Information for Donoghue et al.: Ligand and Substrate Effects on the Mechanisms of the Rhodium...

$\begin{array}{rrrr}\mathrm{H} & -4.81240 & -0.79203 & -3.31604 \\ \mathrm{C} & 2.68261 & 0.38663 & -3.16282 \\ \mathrm{H} & 3.17651 & 0.02126 & -4.06946 \\ \mathrm{H} & 3.47274 & 0.56405 & -2.42181 \\ \mathrm{C} & 1.90140 & 1.68423 & -3.41775 \\ \mathrm{H} & 1.24095 & 1.56306 & -4.28644 \\ \mathrm{H} & 2.56649 & 2.52484 & -3.64272 \\ \mathrm{C} & -3.68866 & -1.64645 & 2.06155 \\ \mathrm{H} & -4.48681 & -1.72286 & 1.31105 \\ \mathrm{H} & -3.92245 & -2.37635 & 2.84461 \\ \mathrm{C} & -3.61588 & -0.21611 & 2.61446 \\ \mathrm{H} & -4.59108 & 0.14354 & 2.96006 \\ \mathrm{H} & -2.93929 & -0.18798 & 3.47868 \\ \mathrm{C} & 2.33650 & -1.92090 & -2.02328 \\ \mathrm{H} & 2.96665 & -2.41307 & -2.77143 \\ \mathrm{H} & 2.96853 & -1.67257 & -1.16638 \\ \mathrm{H} & 1.58683 & -2.64743 & -1.69523 \\ \mathrm{C} & -0.05973 & 3.02043 & -2.35712 \\ \mathrm{H} & 0.36871 & 3.96713 & -2.70213 \\ \mathrm{H} & -0.78873 & 2.69222 & -3.10334 \\ \mathrm{H} & -0.59879 & 3.22391 & -1.42604 \\ \mathrm{C} & -2.29560 & -3.20357 & 0.51270 \\ \mathrm{H} & -2.58990 & -4.08798 & 1.08736 \\ \mathrm{H} & -2.98496 & -3.11000 & -0.33137 \\ \mathrm{H} & -1.29434 & -3.39067 & 0.11125 \\ \mathrm{C} & -2.54329 & 2.05735 & 2.00018 \\ \mathrm{H} & -3.33756 & 2.60292 & 2.52082 \\ \mathrm{H} & -1.71195 & 1.92450 & 2.70011 \\ \mathrm{H} & -2.20065 & 2.69019 & 1.17436\end{array}$

\section{DuPHOS Ligand, Substrate a, Minor Manifold}

\begin{tabular}{|c|c|c|c|}
\hline \multicolumn{2}{|c|}{ SCF Energy } & \multicolumn{2}{|c|}{-1834.471034 hartree } \\
\hline Total & 1 Enthalpy $=-$ & 34369 hart & \\
\hline $\mathrm{Rh}$ & 0.70733 & -0.44377 & 0.85089 \\
\hline 0 & 0.77430 & -0.24798 & 3.03898 \\
\hline $\mathrm{C}$ & 1.90533 & -0.48916 & 3.50808 \\
\hline $\mathrm{N}$ & 2.93916 & -0.85630 & 2.74281 \\
\hline $\mathrm{C}$ & 2.79411 & -0.98829 & 1.30222 \\
\hline $\mathrm{C}$ & 2.16175 & -2.12244 & 0.77003 \\
\hline $\mathrm{C}$ & 3.71224 & -0.17465 & 0.55872 \\
\hline $\mathrm{N}$ & 4.47283 & 0.48970 & -0.01963 \\
\hline $\mathrm{H}$ & 1.80302 & -2.89745 & 1.44194 \\
\hline $\mathrm{H}$ & 2.37676 & -2.42608 & -0.24664 \\
\hline $\mathrm{H}$ & 3.84428 & -1.04446 & 3.16520 \\
\hline $\mathrm{H}$ & 2.09916 & -0.42144 & 4.58433 \\
\hline $\mathrm{P}$ & -1.37518 & 0.65402 & 0.87584 \\
\hline $\mathrm{P}$ & 0.41241 & -0.45969 & -1.43843 \\
\hline $\mathrm{C}$ & -1.91670 & 0.99991 & -0.83827 \\
\hline $\mathrm{C}$ & -1.10185 & 0.51435 & -1.87752 \\
\hline $\mathrm{C}$ & -2.72871 & -0.22720 & 1.84501 \\
\hline $\mathrm{C}$ & -1.51790 & 2.27515 & 1.83145 \\
\hline
\end{tabular}


Supporting Information for Donoghue et al.: Ligand and Substrate Effects on the Mechanisms of the Rhodium...

\begin{tabular}{|c|c|c|c|}
\hline C & 0.18676 & -2.06715 & -2.41407 \\
\hline $\mathrm{C}$ & 1.85884 & 0.13960 & -2.48851 \\
\hline C & -1.48008 & 0.75258 & -3.20875 \\
\hline C & -3.08707 & 1.71421 & -1.14187 \\
\hline C & -3.66745 & -1.12517 & 1.03080 \\
\hline C & -0.19885 & 2.99394 & 2.11546 \\
\hline C & -0.22745 & -3.30880 & -1.62106 \\
\hline C & 1.89878 & 1.63850 & -2.79916 \\
\hline $\mathrm{H}$ & -2.15128 & -0.85127 & 2.54358 \\
\hline $\mathrm{H}$ & -2.13832 & 2.91143 & 1.18572 \\
\hline $\mathrm{H}$ & -0.62698 & -1.82510 & -3.11003 \\
\hline $\mathrm{H}$ & 2.72969 & -0.09324 & -1.86577 \\
\hline C & -3.42367 & 0.89294 & 2.66139 \\
\hline C & -2.34417 & 1.89974 & 3.08937 \\
\hline C & 1.48544 & -2.20547 & -3.23928 \\
\hline $\mathrm{C}$ & 1.89539 & -0.80331 & -3.71258 \\
\hline $\mathrm{H}$ & -4.18472 & 1.38938 & 2.03848 \\
\hline $\mathrm{H}$ & -3.95152 & 0.46799 & 3.52507 \\
\hline $\mathrm{H}$ & -1.68105 & 1.44933 & 3.84464 \\
\hline $\mathrm{H}$ & -2.78482 & 2.79979 & 3.53827 \\
\hline $\mathrm{H}$ & 2.28302 & -2.64001 & -2.62056 \\
\hline $\mathrm{H}$ & 1.33151 & -2.89394 & -4.07752 \\
\hline $\mathrm{H}$ & 1.20494 & -0.44974 & -4.48901 \\
\hline $\mathrm{H}$ & 2.89494 & -0.80116 & -4.15849 \\
\hline $\mathrm{H}$ & 0.32850 & 3.24800 & 1.19132 \\
\hline $\mathrm{H}$ & -0.39284 & 3.92654 & 2.65756 \\
\hline $\mathrm{H}$ & 0.46133 & 2.37972 & 2.72863 \\
\hline $\mathrm{H}$ & -3.12132 & -1.91810 & 0.50484 \\
\hline $\mathrm{H}$ & -4.38609 & -1.60906 & 1.69968 \\
\hline $\mathrm{H}$ & -4.23684 & -0.55471 & 0.28670 \\
\hline $\mathrm{H}$ & 2.80089 & 1.86854 & -3.37541 \\
\hline $\mathrm{H}$ & 1.03445 & 1.96301 & -3.38570 \\
\hline $\mathrm{H}$ & 1.93504 & 2.23690 & -1.88367 \\
\hline $\mathrm{H}$ & -0.39413 & -4.14649 & -2.30538 \\
\hline $\mathrm{H}$ & 0.53849 & -3.61719 & -0.90439 \\
\hline $\mathrm{H}$ & -1.15789 & -3.14435 & -1.06959 \\
\hline C & -2.64073 & 1.46715 & -3.50028 \\
\hline $\mathrm{C}$ & -3.44707 & 1.94874 & -2.46663 \\
\hline $\mathrm{H}$ & -0.87003 & 0.38533 & -4.02786 \\
\hline $\mathrm{H}$ & -2.91589 & 1.64819 & -4.53493 \\
\hline $\mathrm{H}$ & -4.35291 & 2.50260 & -2.69293 \\
\hline $\mathrm{H}$ & -3.72277 & 2.08880 & -0.34499 \\
\hline
\end{tabular}

$3 a-\operatorname{molh}-\mathrm{A}$

SCF Energy = $\quad-1835.646110$ hartree

Total Enthalpy $=-1835.092989$ hartree

$\begin{array}{rrrr}\text { Rh } & -0.05787 & 0.21379 & 0.91860 \\ \mathrm{O} & 1.53632 & -0.29385 & 2.33754 \\ \mathrm{C} & 1.57014 & -1.51952 & 2.57360 \\ \mathrm{~N} & 0.70006 & -2.37172 & 2.03250 \\ \mathrm{C} & -0.45772 & -1.86553 & 1.29816 \\ \mathrm{C} & -1.40253 & -1.07053 & 2.03128 \\ \mathrm{C} & -0.83412 & -2.75207 & 0.23606 \\ \mathrm{~N} & -1.11881 & -3.50953 & -0.60036 \\ \mathrm{H} & -1.27052 & -0.98395 & 3.10593\end{array}$


Supporting Information for Donoghue et al.: Ligand and Substrate Effects on the Mechanisms of the Rhodium...

\begin{tabular}{|c|c|c|c|}
\hline $\mathrm{H}$ & -2.43305 & -1.04756 & 1.69335 \\
\hline $\mathrm{H}$ & 0.74644 & -3.35945 & 2.26002 \\
\hline $\mathrm{H}$ & 2.33222 & -1.94302 & 3.23673 \\
\hline $\mathrm{P}$ & -1.55804 & 0.81879 & -0.74159 \\
\hline $\mathrm{P}$ & 1.62480 & 0.87400 & -0.67175 \\
\hline $\mathrm{C}$ & -0.64639 & 1.84233 & -1.98457 \\
\hline $\mathrm{C}$ & 0.76432 & 1.83070 & -1.98691 \\
\hline $\mathrm{H}$ & 0.04396 & 2.03597 & 1.48767 \\
\hline $\mathrm{H}$ & -0.51362 & 1.70672 & 1.95395 \\
\hline $\mathrm{C}$ & -3.09651 & 1.81398 & -0.25816 \\
\hline $\mathrm{C}$ & -2.51488 & -0.49392 & -1.69851 \\
\hline $\mathrm{C}$ & 3.00898 & 1.91145 & 0.07998 \\
\hline $\mathrm{C}$ & 2.80050 & -0.30055 & -1.57647 \\
\hline $\mathrm{H}$ & 2.81925 & 0.06790 & -2.61023 \\
\hline $\mathrm{H}$ & 3.05874 & 1.51351 & 1.10256 \\
\hline $\mathrm{H}$ & -3.09792 & 2.66444 & -0.94987 \\
\hline $\mathrm{H}$ & -2.60995 & -1.32695 & -0.99397 \\
\hline $\mathrm{C}$ & -0.62717 & 3.30029 & -3.93058 \\
\hline $\mathrm{C}$ & -1.32885 & 2.58087 & -2.96518 \\
\hline $\mathrm{H}$ & -1.17046 & 3.87140 & -4.67730 \\
\hline $\mathrm{H}$ & -2.41357 & 2.59760 & -2.98965 \\
\hline $\mathrm{C}$ & 0.76803 & 3.27957 & -3.93967 \\
\hline $\mathrm{C}$ & 1.45685 & 2.54451 & -2.97730 \\
\hline $\mathrm{H}$ & 1.31805 & 3.83642 & -4.69230 \\
\hline $\mathrm{H}$ & 2.54242 & 2.53496 & -2.99650 \\
\hline $\mathrm{C}$ & -4.28003 & 0.88749 & -0.62376 \\
\hline $\mathrm{H}$ & -4.46191 & 0.18090 & 0.19724 \\
\hline $\mathrm{H}$ & -5.19584 & 1.47652 & -0.74122 \\
\hline $\mathrm{C}$ & -3.91749 & 0.11912 & -1.89982 \\
\hline $\mathrm{H}$ & -4.64300 & -0.67039 & -2.12331 \\
\hline $\mathrm{H}$ & -3.91218 & 0.79567 & -2.76418 \\
\hline $\mathrm{C}$ & 4.29370 & 1.47710 & -0.65682 \\
\hline $\mathrm{H}$ & 5.17898 & 1.71510 & -0.05662 \\
\hline $\mathrm{H}$ & 4.39472 & 2.03068 & -1.59980 \\
\hline $\mathrm{C}$ & 4.18075 & -0.02787 & -0.93726 \\
\hline $\mathrm{H}$ & 4.26551 & -0.58733 & 0.00409 \\
\hline $\mathrm{H}$ & 4.98080 & -0.38704 & -1.59353 \\
\hline $\mathrm{C}$ & -1.84779 & -1.01683 & -2.97199 \\
\hline $\mathrm{H}$ & -2.48252 & -1.78579 & -3.42343 \\
\hline $\mathrm{H}$ & -1.70192 & -0.22272 & -3.70987 \\
\hline $\mathrm{H}$ & -0.88037 & -1.48048 & -2.76291 \\
\hline C & -3.15692 & 2.36228 & 1.16893 \\
\hline $\mathrm{H}$ & -4.11751 & 2.86515 & 1.32312 \\
\hline $\mathrm{H}$ & -3.07429 & 1.56640 & 1.91508 \\
\hline $\mathrm{H}$ & -2.37140 & 3.09830 & 1.36125 \\
\hline $\mathrm{C}$ & 2.38556 & -1.77230 & -1.58648 \\
\hline $\mathrm{H}$ & 3.12301 & -2.36603 & -2.13747 \\
\hline $\mathrm{H}$ & 2.32248 & -2.17743 & -0.57146 \\
\hline $\mathrm{H}$ & 1.41542 & -1.92477 & -2.06754 \\
\hline $\mathrm{C}$ & 2.77137 & 3.42102 & 0.16145 \\
\hline $\mathrm{H}$ & 3.60538 & 3.89819 & 0.68666 \\
\hline $\mathrm{H}$ & 2.69395 & 3.88007 & -0.82808 \\
\hline $\mathrm{H}$ & 1.85794 & 3.66210 & 0.71570 \\
\hline
\end{tabular}

$3 a-\operatorname{molh}-\mathrm{C}$

SCF Energy = $\quad-1835.651128$ hartree 
Supporting Information for Donoghue et al.: Ligand and Substrate Effects on the Mechanisms of the Rhodium...

Total Enthalpy $=-1835.097656$ hartree

\begin{tabular}{|c|c|c|c|}
\hline $\mathrm{Rh}$ & 0.52284 & -0.37650 & 0.07794 \\
\hline 0 & 2.72431 & -0.50294 & 0.03749 \\
\hline C & 3.18860 & -1.01115 & 1.08293 \\
\hline $\mathrm{N}$ & 2.42241 & -1.32285 & 2.12785 \\
\hline C & 1.02347 & -0.92823 & 2.13342 \\
\hline $\mathrm{C}$ & 0.72890 & 0.47545 & 1.99219 \\
\hline $\mathrm{C}$ & 0.15630 & -1.84416 & 2.80110 \\
\hline $\mathrm{N}$ & -0.55474 & -2.59501 & 3.33868 \\
\hline $\mathrm{H}$ & 1.56987 & 1.16521 & 2.01644 \\
\hline $\mathrm{H}$ & -0.18169 & 0.86583 & 2.42387 \\
\hline $\mathrm{H}$ & 2.83945 & -1.72188 & 2.96171 \\
\hline $\mathrm{H}$ & 4.25945 & -1.22186 & 1.17350 \\
\hline P & -1.79022 & -0.36557 & -0.08952 \\
\hline P & 0.44962 & 1.30795 & -1.56393 \\
\hline C & -2.29401 & 0.85976 & -1.38483 \\
\hline $\mathrm{C}$ & -1.29794 & 1.60567 & -2.04607 \\
\hline $\mathrm{H}$ & 0.64707 & -1.76763 & -1.37695 \\
\hline $\mathrm{H}$ & 0.62339 & -2.18375 & -0.72051 \\
\hline C & -3.08938 & -0.17275 & 1.28217 \\
\hline C & -2.40707 & -2.07209 & -0.62464 \\
\hline C & 1.22940 & 2.92647 & -0.98962 \\
\hline C & 1.45453 & 1.20950 & -3.15970 \\
\hline $\mathrm{H}$ & -3.85750 & 0.43413 & 0.78822 \\
\hline $\mathrm{H}$ & -1.68615 & -2.75281 & -0.15415 \\
\hline $\mathrm{H}$ & 0.78159 & 1.56621 & -3.94947 \\
\hline $\mathrm{H}$ & 1.97439 & 2.59042 & -0.25653 \\
\hline C & -3.00484 & 2.70462 & -3.38133 \\
\hline C & -1.66616 & 2.52483 & -3.04103 \\
\hline $\mathrm{H}$ & -3.27659 & 3.42126 & -4.15048 \\
\hline $\mathrm{H}$ & -0.90762 & 3.10834 & -3.55416 \\
\hline C & -3.99212 & 1.96103 & -2.73332 \\
\hline C & -3.63981 & 1.04704 & -1.74236 \\
\hline $\mathrm{H}$ & -5.03658 & 2.08985 & -3.00059 \\
\hline $\mathrm{H}$ & -4.42509 & 0.47424 & -1.25976 \\
\hline $\mathrm{C}$ & 2.57845 & 2.25293 & -2.96331 \\
\hline $\mathrm{H}$ & 3.38673 & 1.81020 & -2.36683 \\
\hline $\mathrm{H}$ & 3.01014 & 2.53226 & -3.93024 \\
\hline C & 1.98769 & 3.46103 & -2.22276 \\
\hline $\mathrm{H}$ & 2.76238 & 4.17289 & -1.91691 \\
\hline $\mathrm{H}$ & 1.29697 & 4.00801 & -2.87833 \\
\hline C & -3.76102 & -2.25578 & 0.09453 \\
\hline $\mathrm{H}$ & -4.00533 & -3.32100 & 0.16669 \\
\hline $\mathrm{H}$ & -4.56208 & -1.78693 & -0.49226 \\
\hline C & -3.65515 & -1.59747 & 1.47534 \\
\hline $\mathrm{H}$ & -2.98759 & -2.17751 & 2.12354 \\
\hline $\mathrm{H}$ & -4.62723 & -1.54394 & 1.97777 \\
\hline C & 1.95047 & -0.19120 & -3.52574 \\
\hline $\mathrm{H}$ & 2.54331 & -0.14870 & -4.44560 \\
\hline $\mathrm{H}$ & 2.58340 & -0.60714 & -2.73617 \\
\hline $\mathrm{H}$ & 1.12074 & -0.88276 & -3.70668 \\
\hline C & 0.28492 & 3.92029 & -0.31142 \\
\hline $\mathrm{H}$ & 0.84777 & 4.79629 & 0.02743 \\
\hline $\mathrm{H}$ & -0.49590 & 4.26895 & -0.99349 \\
\hline $\mathrm{H}$ & -0.20398 & 3.48266 & 0.56488 \\
\hline C & -2.43827 & -2.33613 & -2.13143 \\
\hline
\end{tabular}


Supporting Information for Donoghue et al.: Ligand and Substrate Effects on the Mechanisms of the Rhodium...

$\begin{array}{lrrr}\mathrm{H} & -2.77559 & -3.36121 & -2.31644 \\ \mathrm{H} & -3.12675 & -1.66032 & -2.64674 \\ \mathrm{H} & -1.45161 & -2.22824 & -2.59297 \\ \mathrm{C} & -2.71443 & 0.53408 & 2.58552 \\ \mathrm{H} & -3.61597 & 0.65259 & 3.19638 \\ \mathrm{H} & -2.00362 & -0.04952 & 3.17491 \\ \mathrm{H} & -2.31064 & 1.53671 & 2.41291\end{array}$

$3 a-m o l h-t s-A$

SCF Energy $\quad=-1835.635600$ hartree Total Enthalpy $=-1835.084114$ hartree Negative Frequencies: $-803.93 \mathrm{~cm}^{-1}$

$\begin{array}{rrrr}\mathrm{Rh} & 0.00000 & 0.00000 & 0.00000 \\ \mathrm{O} & 2.20837 & 0.00000 & 0.00000 \\ \mathrm{C} & 2.70886 & 1.14253 & 0.00000 \\ \mathrm{~N} & 1.97356 & 2.25812 & -0.04831 \\ \mathrm{C} & 0.54585 & 2.19071 & -0.29545 \\ \mathrm{C} & 0.11201 & 1.59096 & -1.50738 \\ \mathrm{C} & -0.21612 & 3.18891 & 0.38710 \\ \mathrm{~N} & -0.82423 & 4.01939 & 0.93236 \\ \mathrm{H} & 0.85746 & 1.32640 & -2.24968 \\ \mathrm{H} & -0.86685 & 1.84031 & -1.89897 \\ \mathrm{H} & 2.43466 & 3.16155 & -0.05654 \\ \mathrm{H} & 3.79496 & 1.27670 & 0.05229 \\ \mathrm{P} & -2.28671 & -0.14012 & 0.19576 \\ \mathrm{P} & 0.03746 & -0.55657 & 2.38182 \\ \mathrm{C} & -2.67667 & -0.97752 & 1.79372 \\ \mathrm{C} & -1.67068 & -1.12583 & 2.77112 \\ \mathrm{H} & -0.07347 & -1.58442 & -0.21534 \\ \mathrm{H} & -0.08523 & -0.92695 & -1.29566 \\ \mathrm{C} & -3.28369 & -1.00976 & -1.15054 \\ \mathrm{C} & -3.28166 & 1.46449 & 0.13559 \\ \mathrm{C} & 1.24949 & -1.96520 & 2.70590 \\ \mathrm{C} & 0.59606 & 0.46286 & 3.87774 \\ \mathrm{C} & -1.99879 & -1.70587 & 4.00668 \\ \mathrm{H} & -1.23731 & -1.82643 & 4.77101 \\ \mathrm{C} & -3.98171 & -1.41437 & 2.07485 \\ \mathrm{H} & -4.77425 & -1.29490 & 1.34312 \\ \mathrm{C} & -3.29451 & -2.14444 & 4.27049 \\ \mathrm{H} & -3.52743 & -2.59952 & 5.22847 \\ \mathrm{C} & -4.28804 & -1.99822 & 3.30227 \\ \mathrm{H} & -5.30127 & -2.33334 & 3.50213 \\ \mathrm{C} & 1.91869 & -0.20208 & 4.32280 \\ \mathrm{H} & 2.14600 & 0.06563 & 5.36040 \\ \mathrm{H} & 2.74494 & 0.18014 & 3.70821 \\ \mathrm{C} & 1.78974 & -1.71869 & 4.13004 \\ \mathrm{H} & 2.74804 & -2.23115 & 4.26971 \\ \mathrm{H} & 1.09852 & -2.13782 & 4.87318 \\ \mathrm{C} & -4.50600 & 1.12356 & -0.74474 \\ \mathrm{H} & -4.93109 & 2.04038 & -1.16665 \\ \mathrm{H} & -5.29027 & 0.66785 & -0.12621 \\ \mathrm{C} & -4.05853 & 0.14007 & -1.83323 \\ \mathrm{H} & -3.40544 & 0.64694 & -2.55647 \\ \mathrm{H} & -4.90574 & -0.26017 & -2.39997 \\ \mathrm{C} & -2.52444 & -1.92098 & -2.11504 \\ & & & \end{array}$


Supporting Information for Donoghue et al.: Ligand and Substrate Effects on the Mechanisms of the Rhodium...

$\begin{array}{rrrr}\mathrm{H} & -1.78609 & -1.36417 & -2.70019 \\ \mathrm{H} & -3.23178 & -2.37515 & -2.81717 \\ \mathrm{H} & -2.00782 & -2.73308 & -1.59555 \\ \mathrm{C} & 0.73200 & -3.37887 & 2.43582 \\ \mathrm{H} & 0.38356 & -3.49294 & 1.40442 \\ \mathrm{H} & -0.09073 & -3.64811 & 3.10438 \\ \mathrm{H} & 1.53917 & -4.10235 & 2.59070 \\ \mathrm{C} & 0.69632 & 1.97168 & 3.65325 \\ \mathrm{H} & 1.42797 & 2.21121 & 2.87490 \\ \mathrm{H} & 1.02168 & 2.46398 & 4.57609 \\ \mathrm{H} & -0.25918 & 2.41441 & 3.36119 \\ \mathrm{C} & -3.62225 & 2.09445 & 1.48862 \\ \mathrm{H} & -2.72597 & 2.35085 & 2.05786 \\ \mathrm{H} & -4.24774 & 1.43485 & 2.09641 \\ \mathrm{H} & -4.17426 & 3.02502 & 1.32266 \\ \mathrm{H} & -2.64480 & 2.16357 & -0.41761 \\ \mathrm{H} & -4.00289 & -1.62486 & -0.59649 \\ \mathrm{H} & 2.05461 & -1.74714 & 1.99205 \\ \mathrm{H} & -0.16497 & 0.27074 & 4.64437\end{array}$

$3 a-m o l h-t s-C$

SCF Energy $\quad=-1835.633209$ hartree Total Enthalpy $=-1835.081550$ hartree Negative Frequencies: $-827.36 \mathrm{~cm}^{-1}$

$\begin{array}{rrrr}\mathrm{Rh} & 0.00000 & 0.00000 & 0.00000 \\ \mathrm{O} & 2.20786 & 0.00000 & 0.00000 \\ \mathrm{C} & 2.68150 & 1.15378 & 0.00000 \\ \mathrm{~N} & 1.92328 & 2.24220 & -0.15506 \\ \mathrm{C} & 0.52003 & 2.11204 & -0.50227 \\ \mathrm{C} & 0.18505 & 1.43880 & -1.70118 \\ \mathrm{C} & -0.31570 & 3.10868 & 0.09945 \\ \mathrm{~N} & -0.97783 & 3.93829 & 0.57706 \\ \mathrm{H} & 0.99378 & 1.13126 & -2.35515 \\ \mathrm{H} & -0.76853 & 1.63033 & -2.17120 \\ \mathrm{H} & 2.34448 & 3.16409 & -0.12944 \\ \mathrm{H} & 3.75607 & 1.31864 & 0.13265 \\ \mathrm{P} & -2.29358 & -0.16659 & 0.27747 \\ \mathrm{P} & -0.20332 & -1.97176 & -1.37862 \\ \mathrm{C} & -2.81988 & -1.81138 & -0.38441 \\ \mathrm{C} & -1.91483 & -2.59770 & -1.12537 \\ \mathrm{H} & 0.01661 & -0.96253 & 1.27900 \\ \mathrm{H} & 0.09345 & 0.31438 & 1.57633 \\ \mathrm{C} & -3.63180 & 1.02368 & -0.34997 \\ \mathrm{C} & -2.80304 & -0.00323 & 2.08523 \\ \mathrm{C} & 0.15625 & -1.90163 & -3.23226 \\ \mathrm{C} & 0.95147 & -3.43097 & -1.05431 \\ \mathrm{C} & -4.14862 & 0.75292 & 2.06779 \\ \mathrm{H} & -4.31681 & 1.23775 & 3.03519 \\ \mathrm{H} & -4.97924 & 0.05023 & 1.91960 \\ \mathrm{C} & -4.09964 & 1.76965 & 0.91845 \\ \mathrm{H} & -3.40093 & 2.58136 & 1.15432 \\ \mathrm{H} & -5.07765 & 2.22881 & 0.73745 \\ \mathrm{C} & 1.81806 & -3.51473 & -2.33105 \\ \mathrm{H} & 2.63204 & -2.78013 & -2.27075 \\ \mathrm{H} & 2.28573 & -4.50209 & -40671 \\ & & & \end{array}$


Supporting Information for Donoghue et al.: Ligand and Substrate Effects on the Mechanisms of the Rhodium...

$\begin{array}{lrrr}\mathrm{C} & 0.92259 & -3.20598 & -3.53920 \\ \mathrm{H} & 1.50153 & -3.10064 & -4.46339 \\ \mathrm{H} & 0.21258 & -4.02726 & -3.70389 \\ \mathrm{C} & -4.12858 & -2.27930 & -0.17828 \\ \mathrm{H} & -4.83990 & -1.69045 & 0.39218 \\ \mathrm{C} & -2.34105 & -3.83157 & -1.64288 \\ \mathrm{H} & -1.65730 & -4.44724 & -2.21924 \\ \mathrm{C} & -3.64013 & -4.28564 & -1.42870 \\ \mathrm{H} & -3.95300 & -5.24272 & -1.83504 \\ \mathrm{C} & -4.53473 & -3.50877 & -0.69191 \\ \mathrm{H} & -5.54736 & -3.85906 & -0.51636 \\ \mathrm{C} & 1.74471 & -3.37188 & 0.25330 \\ \mathrm{H} & 1.08502 & -3.36078 & 1.12630 \\ \mathrm{H} & 2.38067 & -2.48420 & 0.29639 \\ \mathrm{H} & 2.38450 & -4.25737 & 0.33493 \\ \mathrm{C} & -1.03877 & -1.60856 & -4.14189 \\ \mathrm{H} & -1.52294 & -0.65977 & -3.88781 \\ \mathrm{H} & -1.79556 & -2.39629 & -4.08601 \\ \mathrm{H} & -0.70549 & -1.53922 & -5.18271 \\ \mathrm{C} & -2.77120 & -1.28619 & 2.91731 \\ \mathrm{H} & -1.77779 & -1.74596 & 2.91851 \\ \mathrm{H} & -3.48801 & -2.02865 & 2.55584 \\ \mathrm{H} & -3.02670 & -1.05362 & 3.95606 \\ \mathrm{C} & -3.34126 & 1.91978 & -1.55309 \\ \mathrm{H} & -2.59567 & 2.68339 & -1.32210 \\ \mathrm{H} & -4.26011 & 2.44302 & -1.83899 \\ \mathrm{H} & -3.01649 & 1.34389 & -2.42583 \\ \mathrm{H} & 0.29295 & -4.30839 & -1.02822 \\ \mathrm{H} & 0.86944 & -1.07112 & -3.31395 \\ \mathrm{H} & -4.42622 & 0.32365 & -0.63959 \\ \mathrm{H} & -2.04280 & 0.68504 & 2.47340 \\ & & & \end{array}$

$3 a-d i h y-A$

SCF Energy $\quad=-1835.640383$ hartree

Total Enthalpy $=-1835.086443$ hartree

$\begin{array}{rrrr}\text { Rh } & -0.08324 & 0.91463 & 0.01550 \\ \mathrm{O} & -0.54157 & 0.71517 & -2.16150 \\ \mathrm{C} & 0.45124 & 0.66109 & -2.90729 \\ \mathrm{~N} & 1.71120 & 0.83028 & -2.47342 \\ \mathrm{C} & 1.98770 & 1.25820 & -1.12479 \\ \mathrm{C} & 1.44948 & 2.46448 & -0.67126 \\ \mathrm{C} & 3.12316 & 0.65565 & -0.50250 \\ \mathrm{~N} & 4.08319 & 0.20605 & -0.02072 \\ \mathrm{H} & 0.93646 & 3.11073 & -1.37331 \\ \mathrm{H} & 1.86115 & 2.93917 & 0.20985 \\ \mathrm{H} & 2.47780 & 0.76905 & -3.13415 \\ \mathrm{H} & 0.34072 & 0.45649 & -3.97844 \\ \mathrm{P} & 0.16900 & 0.96464 & 2.28382 \\ \mathrm{P} & -0.29170 & -1.52053 & 0.25788 \\ \mathrm{C} & -0.29369 & -0.69218 & 2.95106 \\ \mathrm{C} & -0.46666 & -1.78477 & 2.07521 \\ \mathrm{H} & -1.57355 & 0.78187 & 0.43559 \\ \mathrm{H} & -0.67122 & 2.37328 & 0.12015 \\ \mathrm{C} & -0.75256 & 2.25492 & 3.29920 \\ \mathrm{C} & 1.88186 & 1.40913 & 2.95234\end{array}$


Supporting Information for Donoghue et al.: Ligand and Substrate Effects on the Mechanisms of the Rhodium...

\begin{tabular}{|c|c|c|c|}
\hline C & -1.88461 & -2.07552 & -0.59357 \\
\hline $\mathrm{C}$ & 0.72689 & -2.99001 & -0.37635 \\
\hline $\mathrm{H}$ & 0.75087 & -3.69072 & 0.46727 \\
\hline $\mathrm{H}$ & -1.91743 & -1.42279 & -1.47545 \\
\hline $\mathrm{H}$ & -1.09268 & 1.70960 & 4.18802 \\
\hline $\mathrm{H}$ & 2.35970 & 1.95995 & 2.13395 \\
\hline C & -0.75611 & -2.14189 & 4.84930 \\
\hline C & -0.43684 & -0.88742 & 4.33474 \\
\hline $\mathrm{H}$ & -0.86521 & -2.27390 & 5.92143 \\
\hline $\mathrm{H}$ & -0.29301 & -0.06286 & 5.02568 \\
\hline C & -0.93134 & -3.22244 & 3.98517 \\
\hline C & -0.78279 & -3.04354 & 2.61154 \\
\hline $\mathrm{H}$ & -1.18448 & -4.20227 & 4.37874 \\
\hline $\mathrm{H}$ & -0.92545 & -3.89580 & 1.95453 \\
\hline C & -1.61393 & -3.51880 & -1.06682 \\
\hline $\mathrm{H}$ & -2.29431 & -3.78737 & -1.88249 \\
\hline $\mathrm{H}$ & -1.81028 & -4.22475 & -0.24900 \\
\hline C & -0.14391 & -3.60371 & -1.49529 \\
\hline $\mathrm{H}$ & 0.00540 & -3.04046 & -2.42660 \\
\hline $\mathrm{H}$ & 0.17073 & -4.63380 & -1.69555 \\
\hline $\mathrm{C}$ & 0.36079 & 3.23259 & 3.73693 \\
\hline $\mathrm{H}$ & 0.59519 & 3.92006 & 2.91293 \\
\hline $\mathrm{H}$ & 0.01632 & 3.84876 & 4.57405 \\
\hline $\mathrm{C}$ & 1.59784 & 2.40198 & 4.10439 \\
\hline $\mathrm{H}$ & 2.47738 & 3.02853 & 4.28659 \\
\hline $\mathrm{H}$ & 1.41119 & 1.84365 & 5.03121 \\
\hline $\mathrm{C}$ & -1.96864 & 2.91453 & 2.64901 \\
\hline $\mathrm{H}$ & -2.43100 & 3.60676 & 3.36061 \\
\hline $\mathrm{H}$ & -1.68976 & 3.48330 & 1.75726 \\
\hline $\mathrm{H}$ & -2.72576 & 2.18129 & 2.35624 \\
\hline C & 2.78748 & 0.23338 & 3.33092 \\
\hline $\mathrm{H}$ & 3.74382 & 0.61978 & 3.69856 \\
\hline $\mathrm{H}$ & 2.34738 & -0.37853 & 4.12308 \\
\hline $\mathrm{H}$ & 3.00655 & -0.40864 & 2.47436 \\
\hline C & -3.17955 & -1.88602 & 0.19814 \\
\hline $\mathrm{H}$ & -4.03340 & -2.18821 & -0.41718 \\
\hline $\mathrm{H}$ & -3.19306 & -2.49158 & 1.10899 \\
\hline $\mathrm{H}$ & -3.33398 & -0.84015 & 0.48088 \\
\hline $\mathrm{C}$ & 2.16438 & -2.68778 & -0.79639 \\
\hline $\mathrm{H}$ & 2.64848 & -3.60732 & -1.14270 \\
\hline $\mathrm{H}$ & 2.19705 & -1.96793 & -1.62043 \\
\hline $\mathrm{H}$ & 2.76402 & -2.28623 & 0.02340 \\
\hline
\end{tabular}

3a-dihy-c

SCF Energy $\quad=-1835.637619$ hartree Total Enthalpy $=-1835.083496$ hartree

$\begin{array}{rrrr}\text { Rh } & 0.60423 & -0.40682 & -0.10521 \\ \mathrm{O} & 0.37214 & -0.60016 & -2.30898 \\ \mathrm{C} & 1.44786 & -0.72169 & -2.91459 \\ \mathrm{~N} & 2.64305 & -0.53600 & -2.33051 \\ \mathrm{C} & 2.75227 & -0.04691 & -0.97652 \\ \mathrm{C} & 2.24759 & 1.20307 & -0.65258 \\ \mathrm{C} & 3.74116 & -0.72251 & -0.18257 \\ \mathrm{~N} & 4.57624 & -1.25098 & 0.43069\end{array}$


Supporting Information for Donoghue et al.: Ligand and Substrate Effects on the Mechanisms of the Rhodium...

\begin{tabular}{|c|c|c|c|}
\hline $\mathrm{H}$ & 1.81865 & 1.81599 & -1.43536 \\
\hline $\mathrm{H}$ & 2.52883 & 1.68440 & 0.27310 \\
\hline $\mathrm{H}$ & 3.49274 & -0.70195 & -2.85762 \\
\hline $\mathrm{H}$ & 1.47248 & -0.99345 & -3.97614 \\
\hline $\mathrm{P}$ & 0.62485 & -0.59699 & 2.18365 \\
\hline $\mathrm{P}$ & -1.06373 & 1.35983 & 0.21854 \\
\hline $\mathrm{C}$ & -0.84087 & 0.32389 & 2.82932 \\
\hline $\mathrm{C}$ & -1.57166 & 1.18489 & 1.98416 \\
\hline $\mathrm{H}$ & -0.64245 & -1.30464 & 0.13240 \\
\hline $\mathrm{H}$ & 1.18398 & -1.88057 & -0.12907 \\
\hline $\mathrm{C}$ & 2.02407 & -0.07864 & 3.35065 \\
\hline $\mathrm{C}$ & 0.60663 & -2.38542 & 2.77891 \\
\hline $\mathrm{C}$ & -0.75606 & 3.19629 & -0.12891 \\
\hline $\mathrm{C}$ & -2.66183 & 1.28813 & -0.79009 \\
\hline $\mathrm{H}$ & 1.46130 & 0.30652 & 4.21126 \\
\hline $\mathrm{H}$ & 1.12594 & -2.89377 & 1.95920 \\
\hline $\mathrm{H}$ & -3.46522 & 1.37899 & -0.04857 \\
\hline $\mathrm{H}$ & 0.00603 & 3.17715 & -0.91910 \\
\hline $\mathrm{C}$ & -3.03281 & 1.75216 & 3.84890 \\
\hline $\mathrm{C}$ & -2.66435 & 1.89219 & 2.51273 \\
\hline $\mathrm{H}$ & -3.88219 & 2.30684 & 4.23605 \\
\hline $\mathrm{H}$ & -3.23801 & 2.56193 & 1.87957 \\
\hline $\mathrm{C}$ & -2.30896 & 0.90094 & 4.68443 \\
\hline $\mathrm{C}$ & -1.21886 & 0.19742 & 4.17703 \\
\hline $\mathrm{H}$ & -2.59133 & 0.78402 & 5.72612 \\
\hline $\mathrm{H}$ & -0.66527 & -0.45888 & 4.84125 \\
\hline $\mathrm{C}$ & 1.53332 & -2.41919 & 4.01339 \\
\hline $\mathrm{H}$ & 1.91180 & -3.43495 & 4.16856 \\
\hline $\mathrm{H}$ & 0.97454 & -2.14943 & 4.91943 \\
\hline $\mathrm{C}$ & 2.67075 & -1.41363 & 3.77573 \\
\hline $\mathrm{H}$ & 3.34466 & -1.77311 & 2.98821 \\
\hline $\mathrm{H}$ & 3.27808 & -1.26370 & 4.67504 \\
\hline $\mathrm{C}$ & -2.63594 & 2.58220 & -1.63374 \\
\hline $\mathrm{H}$ & -2.00092 & 2.43279 & -2.51739 \\
\hline $\mathrm{H}$ & -3.64102 & 2.81393 & -2.00197 \\
\hline $\mathrm{C}$ & -2.06574 & 3.71057 & -0.76410 \\
\hline $\mathrm{H}$ & -1.87779 & 4.62155 & -1.34328 \\
\hline $\mathrm{H}$ & -2.78196 & 3.98060 & 0.02295 \\
\hline $\mathrm{C}$ & -2.88247 & 0.00736 & -1.59843 \\
\hline $\mathrm{H}$ & -3.82694 & 0.07946 & -2.14902 \\
\hline $\mathrm{H}$ & -2.07761 & -0.15870 & -2.31859 \\
\hline $\mathrm{H}$ & -2.94358 & -0.87360 & -0.95275 \\
\hline $\mathrm{C}$ & -0.22290 & 4.03177 & 1.03739 \\
\hline $\mathrm{H}$ & -0.03614 & 5.05824 & 0.70421 \\
\hline $\mathrm{H}$ & -0.93560 & 4.07470 & 1.86580 \\
\hline $\mathrm{H}$ & 0.72079 & 3.63712 & 1.42908 \\
\hline $\mathrm{C}$ & -0.76790 & -3.03312 & 2.95039 \\
\hline $\mathrm{H}$ & -0.64300 & -4.08551 & 3.22477 \\
\hline $\mathrm{H}$ & -1.35865 & -2.55225 & 3.73464 \\
\hline $\mathrm{H}$ & -1.34605 & -3.00198 & 2.02141 \\
\hline $\mathrm{C}$ & 2.99660 & 1.01239 & 2.90591 \\
\hline $\mathrm{H}$ & 3.65619 & 1.27207 & 3.74082 \\
\hline $\mathrm{H}$ & 3.63489 & 0.68052 & 2.08468 \\
\hline $\mathrm{H}$ & 2.47452 & 1.93037 & 2.61449 \\
\hline
\end{tabular}

$3 a-d i h y-t s-A$ 
Supporting Information for Donoghue et al.: Ligand and Substrate Effects on the Mechanisms of the Rhodium...

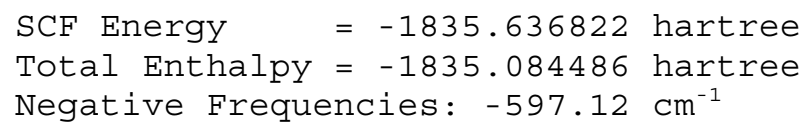

\begin{tabular}{|c|c|c|c|}
\hline $\mathrm{Rh}$ & 0.00000 & 0.00000 & 0.00000 \\
\hline 0 & 2.22975 & 0.00000 & 0.00000 \\
\hline $\mathrm{C}$ & 2.75452 & 1.13044 & 0.00000 \\
\hline $\mathrm{N}$ & 2.05007 & 2.26467 & -0.11314 \\
\hline $\mathrm{C}$ & 0.63555 & 2.23112 & -0.39411 \\
\hline $\mathrm{C}$ & 0.21465 & 1.62148 & -1.60749 \\
\hline $\mathrm{C}$ & -0.15265 & 3.21933 & 0.26310 \\
\hline $\mathrm{N}$ & -0.78655 & 4.05182 & 0.77728 \\
\hline $\mathrm{H}$ & 0.97797 & 1.40112 & -2.34756 \\
\hline $\mathrm{H}$ & -0.75212 & 1.89853 & -2.01200 \\
\hline $\mathrm{H}$ & 2.53349 & 3.15607 & -0.09843 \\
\hline $\mathrm{H}$ & 3.83954 & 1.24565 & 0.10081 \\
\hline $\mathrm{P}$ & -2.26610 & -0.23853 & 0.13901 \\
\hline $\mathrm{P}$ & 0.04080 & -0.55823 & 2.32415 \\
\hline $\mathrm{C}$ & -2.65177 & -1.07703 & 1.73355 \\
\hline $\mathrm{C}$ & -1.64214 & -1.19507 & 2.71040 \\
\hline $\mathrm{H}$ & -0.11044 & -1.55463 & -0.12177 \\
\hline $\mathrm{H}$ & -0.09056 & -0.09195 & -1.60741 \\
\hline $\mathrm{C}$ & -3.20209 & -1.14563 & -1.22087 \\
\hline $\mathrm{C}$ & -3.29982 & 1.33853 & 0.03915 \\
\hline $\mathrm{C}$ & 1.32018 & -1.90183 & 2.64715 \\
\hline $\mathrm{C}$ & 0.55026 & 0.50332 & 3.80434 \\
\hline $\mathrm{C}$ & -1.94690 & -1.79526 & 3.94181 \\
\hline $\mathrm{H}$ & -1.18151 & -1.89350 & 4.70559 \\
\hline $\mathrm{C}$ & -3.94008 & -1.56176 & 2.00959 \\
\hline $\mathrm{H}$ & -4.73446 & -1.46861 & 1.27579 \\
\hline $\mathrm{C}$ & -3.22611 & -2.28367 & 4.20017 \\
\hline $\mathrm{H}$ & -3.44241 & -2.75587 & 5.15369 \\
\hline $\mathrm{C}$ & -4.22418 & -2.16632 & 3.23260 \\
\hline $\mathrm{H}$ & -5.22369 & -2.54204 & 3.42915 \\
\hline $\mathrm{C}$ & 1.91267 & -0.08348 & 4.23819 \\
\hline $\mathrm{H}$ & 2.13792 & 0.20660 & 5.27013 \\
\hline $\mathrm{H}$ & 2.70896 & 0.33668 & 3.60906 \\
\hline $\mathrm{C}$ & 1.86227 & -1.60751 & 4.06225 \\
\hline $\mathrm{H}$ & 2.84780 & -2.06681 & 4.19608 \\
\hline $\mathrm{H}$ & 1.20249 & -2.05340 & 4.81837 \\
\hline $\mathrm{C}$ & -4.50406 & 0.94841 & -0.84763 \\
\hline $\mathrm{H}$ & -4.95793 & 1.84506 & -1.28291 \\
\hline $\mathrm{H}$ & -5.27730 & 0.47059 & -0.23165 \\
\hline $\mathrm{C}$ & -4.00907 & -0.02891 & -1.92217 \\
\hline $\mathrm{H}$ & -3.36488 & 0.49666 & -2.64035 \\
\hline $\mathrm{H}$ & -4.83397 & -0.46201 & -2.49749 \\
\hline C & 0.87042 & -3.34327 & 2.40192 \\
\hline $\mathrm{H}$ & 0.52398 & -3.48840 & 1.37423 \\
\hline $\mathrm{H}$ & 0.06578 & -3.64328 & 3.07933 \\
\hline $\mathrm{H}$ & 1.71387 & -4.02265 & 2.56327 \\
\hline $\mathrm{C}$ & 0.55560 & 2.01405 & 3.57244 \\
\hline $\mathrm{H}$ & 1.26574 & 2.29537 & 2.78864 \\
\hline $\mathrm{H}$ & 0.85414 & 2.53017 & 4.49132 \\
\hline $\mathrm{H}$ & -0.42719 & 2.39508 & 3.28362 \\
\hline $\mathrm{C}$ & -3.66382 & 1.99016 & 1.37537 \\
\hline $\mathrm{H}$ & -2.77568 & 2.29472 & 1.93475 \\
\hline $\mathrm{H}$ & -4.26192 & 1.32289 & 2.00244 \\
\hline
\end{tabular}


Supporting Information for Donoghue et al.: Ligand and Substrate Effects on the Mechanisms of the Rhodium...

$\begin{array}{rrrr}\mathrm{H} & -4.25186 & 2.89433 & 1.18844 \\ \mathrm{C} & -2.37153 & -2.01972 & -2.16064 \\ \mathrm{H} & -1.82507 & -2.79807 & -1.62121 \\ \mathrm{H} & -1.64264 & -1.42773 & -2.72221 \\ \mathrm{H} & -3.03310 & -2.51133 & -2.88162 \\ \mathrm{H} & 2.10371 & -1.65427 & 1.91986 \\ \mathrm{H} & -2.67130 & 2.03990 & -0.52300 \\ \mathrm{H} & -3.90756 & -1.79094 & -0.68338 \\ \mathrm{H} & -0.19116 & 0.26753 & 4.57832\end{array}$

$3 a-d i h y-t s-c$

SCF Energy = -1835.613759 hartree

Total Enthalpy $=-1835.062452$ hartree

Negative Frequencies: $-744.89 \mathrm{~cm}^{-1}$

\begin{tabular}{|c|c|c|c|}
\hline $\mathrm{Rh}$ & 0.00000 & 0.00000 & 0.00000 \\
\hline 0 & 2.26442 & 0.00000 & 0.00000 \\
\hline $\mathrm{C}$ & 2.78314 & 1.12671 & 0.00000 \\
\hline $\mathrm{N}$ & 2.08014 & 2.25773 & -0.17542 \\
\hline $\mathrm{C}$ & 0.66170 & 2.18681 & -0.50428 \\
\hline $\mathrm{C}$ & 0.31344 & 1.46211 & -1.69836 \\
\hline $\mathrm{C}$ & -0.03540 & 3.42688 & -0.20694 \\
\hline $\mathrm{N}$ & -0.59024 & 4.42397 & 0.00758 \\
\hline $\mathrm{H}$ & 1.13439 & 1.11378 & -2.31432 \\
\hline $\mathrm{H}$ & -0.59721 & 1.73463 & -2.21368 \\
\hline $\mathrm{H}$ & 2.53734 & 3.16078 & -0.14996 \\
\hline $\mathrm{H}$ & 3.86049 & 1.25687 & 0.15575 \\
\hline $\mathrm{P}$ & -2.21735 & -0.11623 & 0.53252 \\
\hline $\mathrm{P}$ & -0.28628 & -2.04088 & -1.07933 \\
\hline $\mathrm{C}$ & -2.81403 & -1.80207 & 0.08520 \\
\hline $\mathrm{C}$ & -1.95978 & -2.65889 & -0.63509 \\
\hline $\mathrm{H}$ & 0.00762 & -0.76551 & 1.39418 \\
\hline $\mathrm{H}$ & 0.21521 & 1.51080 & 0.74156 \\
\hline $\mathrm{C}$ & -3.56351 & 1.04930 & -0.11828 \\
\hline $\mathrm{C}$ & -2.57602 & 0.25022 & 2.34245 \\
\hline $\mathrm{C}$ & -0.05958 & -2.12174 & -2.95065 \\
\hline $\mathrm{C}$ & 0.92811 & -3.43451 & -0.70832 \\
\hline $\mathrm{C}$ & -3.91152 & 1.02298 & 2.34892 \\
\hline $\mathrm{H}$ & -4.00656 & 1.60504 & 3.27169 \\
\hline $\mathrm{H}$ & -4.75673 & 0.32226 & 2.33154 \\
\hline $\mathrm{C}$ & -3.93903 & 1.91872 & 1.10160 \\
\hline $\mathrm{H}$ & -3.21841 & 2.73966 & 1.20925 \\
\hline $\mathrm{H}$ & -4.92178 & 2.37697 & 0.94680 \\
\hline $\mathrm{C}$ & 1.71796 & -3.59603 & -2.02634 \\
\hline $\mathrm{H}$ & 2.50452 & -2.83156 & -2.07927 \\
\hline $\mathrm{H}$ & 2.21812 & -4.56999 & -2.05055 \\
\hline $\mathrm{C}$ & 0.73634 & -3.42212 & -3.19499 \\
\hline $\mathrm{H}$ & 1.25112 & -3.37793 & -4.16104 \\
\hline $\mathrm{H}$ & 0.04980 & -4.27797 & -3.24156 \\
\hline $\mathrm{C}$ & -4.10221 & -2.24174 & 0.42970 \\
\hline $\mathrm{H}$ & -4.77436 & -1.59447 & 0.98450 \\
\hline $\mathrm{C}$ & -2.40955 & -3.93925 & -0.99299 \\
\hline $\mathrm{H}$ & -1.76464 & -4.61050 & -1.55217 \\
\hline C & -3.68780 & -4.36710 & -0.63960 \\
\hline $\mathrm{H}$ & -4.02170 & -5.36111 & -0.92136 \\
\hline $\mathrm{C}$ & -4.53500 & -3.51809 & 0.07419 \\
\hline
\end{tabular}


Supporting Information for Donoghue et al.: Ligand and Substrate Effects on the Mechanisms of the Rhodium...

$\begin{array}{rrrr}\mathrm{H} & -5.53017 & -3.84894 & 0.35488 \\ \mathrm{C} & -2.48413 & -0.93636 & 3.30243 \\ \mathrm{H} & -1.49978 & -1.41264 & 3.25740 \\ \mathrm{H} & -3.24161 & -1.69601 & 3.08976 \\ \mathrm{H} & -2.63722 & -0.58963 & 4.32952 \\ \mathrm{C} & -3.28562 & 1.83195 & -1.40099 \\ \mathrm{H} & -2.98536 & 1.17720 & -2.22508 \\ \mathrm{H} & -2.51453 & 2.59202 & -1.25142 \\ \mathrm{H} & -4.19617 & 2.35431 & -1.71368 \\ \mathrm{C} & 1.79027 & -3.26602 & 0.54502 \\ \mathrm{H} & 1.17876 & -3.18582 & 1.44827 \\ \mathrm{H} & 2.41867 & -2.37512 & 0.48182 \\ \mathrm{H} & 2.44123 & -4.13970 & 0.65940 \\ \mathrm{C} & -1.32594 & -1.95234 & -3.79192 \\ \mathrm{H} & -1.82520 & -0.99947 & -3.58720 \\ \mathrm{H} & -2.04603 & -2.75563 & -3.61131 \\ \mathrm{H} & -1.06908 & -1.96715 & -4.85625 \\ \mathrm{H} & -4.39573 & 0.36004 & -0.31234 \\ \mathrm{H} & -1.77475 & 0.95753 & 2.59115 \\ \mathrm{H} & 0.60896 & -1.27511 & -3.15037 \\ \mathrm{H} & 0.29149 & -4.31987 & -0.58142\end{array}$

$3 a-a l h y-A$

SCF Energy $\quad=-1835.682477$ hartree Total Enthalpy $=-1835.125333$ hartree

$\begin{array}{rrrr}\mathrm{Rh} & 0.75621 & -0.11219 & -1.40983 \\ \mathrm{O} & 0.72016 & -0.27335 & -3.56374 \\ \mathrm{C} & 1.87247 & -0.34161 & -4.05204 \\ \mathrm{~N} & 2.96704 & -0.36931 & -3.29832 \\ \mathrm{C} & 2.86992 & -0.47100 & -1.83581 \\ \mathrm{C} & 4.09710 & 0.12895 & -1.17144 \\ \mathrm{C} & 2.53020 & -1.83032 & -1.45344 \\ \mathrm{~N} & 2.02203 & -2.83725 & -1.13335 \\ \mathrm{H} & 5.02521 & -0.32826 & -1.54356 \\ \mathrm{H} & 4.07086 & -0.03000 & -0.09223 \\ \mathrm{H} & 3.87248 & -0.46691 & -3.74868 \\ \mathrm{H} & 2.01053 & -0.37899 & -5.13758 \\ \mathrm{P} & 0.67504 & 0.23334 & 0.86623 \\ \mathrm{P} & -1.60062 & -0.00927 & -1.33492 \\ \mathrm{C} & -1.09541 & 0.41387 & 1.37216 \\ \mathrm{C} & -2.10963 & 0.29716 & 0.40062 \\ \mathrm{H} & 0.85648 & 1.40057 & -1.50564 \\ \mathrm{H} & 4.14537 & 1.20323 & -1.36394 \\ \mathrm{C} & 1.56578 & 1.66699 & 1.71056 \\ \mathrm{C} & 1.47367 & -1.10565 & 1.92428 \\ \mathrm{C} & -2.35970 & 1.27590 & -2.47654 \\ \mathrm{C} & -2.64285 & -1.44384 & -1.97744 \\ \mathrm{H} & 2.27301 & -1.49139 & 1.28068 \\ \mathrm{H} & -1.61656 & 1.34322 & -3.28223 \\ \mathrm{H} & 0.81563 & 2.07699 & 2.39722 \\ \mathrm{H} & -3.42638 & -1.58694 & -1.22229 \\ \mathrm{C} & -2.79053 & 0.77451 & 3.07762 \\ \mathrm{C} & -1.45214 & 0.65532 & 2.70910 \\ \mathrm{H} & -3.04949 & 0.95852 & 4.11597 \\ \mathrm{H} & -0.68894 & 0.74592 & 3.47548 \\ & & & \end{array}$


Supporting Information for Donoghue et al.: Ligand and Substrate Effects on the Mechanisms of the Rhodium...

$\begin{array}{rrrr}\mathrm{C} & -3.45455 & 0.41539 & 0.78539 \\ \mathrm{C} & -3.79407 & 0.65426 & 2.11487 \\ \mathrm{H} & -4.24431 & 0.32405 & 0.04567 \\ \mathrm{H} & -4.83782 & 0.74723 & 2.39949 \\ \mathrm{C} & 2.12477 & -0.33903 & 3.09743 \\ \mathrm{H} & 1.38075 & -0.15265 & 3.88251 \\ \mathrm{H} & 2.91284 & -0.94829 & 3.55329 \\ \mathrm{C} & 2.67081 & 0.99119 & 2.55643 \\ \mathrm{H} & 2.99061 & 1.66073 & 3.36201 \\ \mathrm{H} & 3.55461 & 0.80662 & 1.93287 \\ \mathrm{C} & -3.29142 & -0.88904 & -3.26760 \\ \mathrm{H} & -4.17944 & -1.47564 & -3.52623 \\ \mathrm{H} & -2.58495 & -0.99070 & -4.10128 \\ \mathrm{C} & -3.62225 & 0.59429 & -3.04784 \\ \mathrm{H} & -4.45642 & 0.69806 & -2.34094 \\ \mathrm{H} & -3.93378 & 1.08518 & -3.97641 \\ \mathrm{C} & 0.57608 & -2.28113 & 2.31730 \\ \mathrm{H} & 0.20472 & -2.81294 & 1.43682 \\ \mathrm{H} & -0.27996 & -1.96007 & 2.91760 \\ \mathrm{H} & 1.15296 & -2.99743 & 2.91135 \\ \mathrm{C} & 2.04667 & 2.79622 & 0.79794 \\ \mathrm{H} & 1.21430 & 3.27504 & 0.27350 \\ \mathrm{H} & 2.75579 & 2.43584 & 0.04846 \\ \mathrm{H} & 2.54807 & 3.56617 & 1.39397 \\ \mathrm{C} & -1.88100 & -2.75852 & -2.16408 \\ \mathrm{H} & -1.45421 & -3.12382 & -1.22532 \\ \mathrm{H} & -1.06517 & -2.64525 & -2.88461 \\ \mathrm{H} & -2.55873 & -3.53139 & -2.54203 \\ \mathrm{C} & -2.57067 & 2.66966 & -1.88251 \\ \mathrm{H} & -1.63193 & 3.10477 & -1.52498 \\ \mathrm{H} & -3.27666 & 2.65487 & -1.04711 \\ \mathrm{H} & -2.97347 & 3.34067 & -2.64818\end{array}$

$3 a-a l h y-c$

SCF Energy $\quad=-1835.662848$ hartree Total Enthalpy $=-1835.105396$ hartree

$\begin{array}{rrrr}\mathrm{Rh} & 0.60849 & 0.14371 & -1.32122 \\ \mathrm{O} & 0.56820 & 0.08268 & -3.48704 \\ \mathrm{C} & 1.55099 & 0.14354 & -4.25323 \\ \mathrm{~N} & 2.83303 & 0.01094 & -3.92181 \\ \mathrm{C} & 3.35645 & -0.28805 & -2.56723 \\ \mathrm{C} & 2.68058 & 0.53276 & -1.45403 \\ \mathrm{C} & 4.80769 & -0.03581 & -2.61206 \\ \mathrm{~N} & 5.94952 & 0.17098 & -2.62225 \\ \mathrm{H} & 2.81025 & 1.60226 & -1.67652 \\ \mathrm{H} & 3.23242 & 0.32499 & -0.53429 \\ \mathrm{H} & 3.50676 & 0.05669 & -4.67672 \\ \mathrm{H} & 1.37892 & 0.32191 & -5.32246 \\ \mathrm{P} & -1.75240 & -0.35857 & -1.21130 \\ \mathrm{P} & 0.51170 & 0.34854 & 0.94304 \\ \mathrm{C} & -2.18706 & -0.41607 & 0.57795 \\ \mathrm{C} & -1.19269 & -0.07005 & 1.51867 \\ \mathrm{H} & 0.93481 & -1.29713 & -1.01171 \\ \mathrm{H} & 3.22001 & -1.36037 & -2.37367 \\ \mathrm{C} & -2.47011 & -1.91257 & -2.00521\end{array}$


Supporting Information for Donoghue et al.: Ligand and Substrate Effects on the Mechanisms of the Rhodium...

$\begin{array}{rrrr}\mathrm{C} & -2.93747 & 0.82343 & -2.08807 \\ \mathrm{C} & 1.77366 & -0.60514 & 1.96426 \\ \mathrm{C} & 0.92252 & 2.02739 & 1.69632 \\ \mathrm{H} & -2.32525 & 1.20849 & -2.91511 \\ \mathrm{H} & 2.60236 & -0.73851 & 1.25883 \\ \mathrm{H} & 0.14162 & 2.18872 & 2.45036 \\ \mathrm{H} & -3.12326 & -2.35902 & -1.24513 \\ \mathrm{C} & -2.79407 & -0.38414 & 3.32160 \\ \mathrm{C} & -1.51413 & -0.04854 & 2.88585 \\ \mathrm{H} & -3.02559 & -0.36764 & 4.38236 \\ \mathrm{H} & -0.76433 & 0.22654 & 3.62148 \\ \mathrm{C} & -3.47029 & -0.75669 & 1.03425 \\ \mathrm{C} & -3.77271 & -0.74479 & 2.39416 \\ \mathrm{H} & -4.24561 & -1.02962 & 0.32464 \\ \mathrm{H} & -4.77077 & -1.01070 & 2.72935 \\ \mathrm{C} & 2.23697 & 0.39231 & 3.05076 \\ \mathrm{H} & 1.54343 & 0.37195 & 3.90153 \\ \mathrm{H} & 3.21812 & 0.09935 & 3.43964 \\ \mathrm{C} & 2.26613 & 1.79543 & 2.42458 \\ \mathrm{H} & 2.42972 & 2.57613 & 3.17511 \\ \mathrm{H} & 3.09288 & 1.86758 & 1.70602 \\ \mathrm{C} & -3.34978 & -1.37972 & -3.16019 \\ \mathrm{H} & -4.07611 & -2.14024 & -3.46648 \\ \mathrm{H} & -2.71711 & -1.17122 & -4.03296 \\ \mathrm{C} & -4.02917 & -0.08522 & -2.69340 \\ \mathrm{H} & -4.79110 & -0.30825 & -1.93462 \\ \mathrm{H} & -4.54293 & 0.42671 & -3.51478 \\ \mathrm{C} & 1.34941 & -1.98660 & 2.46857 \\ \mathrm{H} & 1.06608 & -2.65026 & 1.64534 \\ \mathrm{H} & 0.50616 & -1.93183 & 3.16205 \\ \mathrm{H} & 2.18586 & -2.45599 & 2.99658 \\ \mathrm{C} & 0.91175 & 3.20362 & 0.71674 \\ \mathrm{H} & -0.06606 & 3.32737 & 0.23909 \\ \mathrm{H} & 1.66541 & 3.07977 & -0.06699 \\ \mathrm{H} & 1.13557 & 4.13553 & 1.24649 \\ \mathrm{C} & -1.42711 & -2.94832 & -2.43426 \\ \mathrm{H} & -0.85814 & -3.33046 & -1.58101 \\ \mathrm{H} & -0.71985 & -2.52182 & -3.15227 \\ \mathrm{H} & -1.92050 & -3.80207 & -2.91110 \\ \mathrm{C} & -3.45186 & 2.00722 & -1.26597 \\ \mathrm{H} & -2.63431 & 2.63127 & -0.88944 \\ \mathrm{H} & -4.04620 & 1.67925 & -0.40820 \\ \mathrm{H} & -4.08855 & 2.64360 & -1.88962\end{array}$

\section{TMBOP Ligand, Substrate a, Major Manifold}

\begin{tabular}{|c|c|c|c|}
\hline \multicolumn{2}{|c|}{ SCF Energy } & \multicolumn{2}{|c|}{-1832.454186 hartree } \\
\hline Total & 1 Enthalpy $=$ - & \multicolumn{2}{|c|}{-1831.932075 hartree } \\
\hline $\mathrm{Rh}$ & 0.69444 & -0.07199 & 0.90897 \\
\hline $\mathrm{O}$ & 0.56685 & -0.43508 & 3.06988 \\
\hline $\mathrm{C}$ & 1.68590 & -0.52878 & 3.61476 \\
\hline $\mathrm{N}$ & 2.83059 & -0.33114 & 2.95437 \\
\hline
\end{tabular}


Supporting Information for Donoghue et al.: Ligand and Substrate Effects on the Mechanisms of the Rhodium...

\begin{tabular}{|c|c|c|c|}
\hline $\mathrm{C}$ & 2.82099 & 0.07463 & 1.56129 \\
\hline C & 2.28476 & 1.33881 & 1.21190 \\
\hline $\mathrm{C}$ & 3.77567 & -0.60177 & 0.73961 \\
\hline $\mathrm{N}$ & 4.55870 & -1.14067 & 0.06653 \\
\hline $\mathrm{H}$ & 1.97628 & 2.01394 & 2.00591 \\
\hline $\mathrm{H}$ & 2.61147 & 1.81309 & 0.29529 \\
\hline $\mathrm{H}$ & 3.71480 & -0.41172 & 3.44424 \\
\hline $\mathrm{H}$ & 1.77388 & -0.77786 & 4.67804 \\
\hline $\mathrm{P}$ & 0.66460 & -0.08327 & -1.38386 \\
\hline $\mathrm{P}$ & -1.62947 & 0.26347 & 0.69714 \\
\hline C & 2.01986 & 0.67332 & -2.42457 \\
\hline $\mathrm{C}$ & 0.58847 & -1.85468 & -2.00159 \\
\hline $\mathrm{C}$ & -2.25318 & 1.92376 & 1.30201 \\
\hline $\mathrm{C}$ & -2.72914 & -0.91762 & 1.63461 \\
\hline $\mathrm{H}$ & 2.94935 & 0.33697 & -1.94932 \\
\hline $\mathrm{H}$ & -2.33420 & -0.87180 & 2.65728 \\
\hline $\mathrm{H}$ & -0.43130 & -2.20065 & -1.78785 \\
\hline $\mathrm{H}$ & -1.93839 & 2.65722 & 0.54785 \\
\hline C & -0.93573 & 0.68106 & -1.97240 \\
\hline $\mathrm{H}$ & -0.82232 & 1.76654 & -1.88326 \\
\hline $\mathrm{H}$ & -1.10966 & 0.46185 & -3.03100 \\
\hline C & -2.11038 & 0.19452 & -1.10733 \\
\hline $\mathrm{H}$ & -2.36057 & -0.84377 & -1.34764 \\
\hline $\mathrm{H}$ & -3.00805 & 0.78902 & -1.30185 \\
\hline C & -3.79860 & 1.90590 & 1.35043 \\
\hline $\mathrm{H}$ & -4.14992 & 2.85838 & 1.76066 \\
\hline $\mathrm{H}$ & -4.22529 & 1.80426 & 0.33960 \\
\hline $\mathrm{C}$ & -4.18652 & -0.41161 & 1.67457 \\
\hline $\mathrm{H}$ & -4.77035 & -1.06200 & 2.33394 \\
\hline $\mathrm{H}$ & -4.63760 & -0.46615 & 0.66926 \\
\hline C & 2.00617 & 0.10374 & -3.86500 \\
\hline $\mathrm{H}$ & 2.91092 & 0.44460 & -4.37816 \\
\hline $\mathrm{H}$ & 1.13944 & 0.50415 & -4.41781 \\
\hline C & 0.79531 & -1.89444 & -3.53144 \\
\hline $\mathrm{H}$ & 0.83512 & -2.93970 & -3.85501 \\
\hline $\mathrm{H}$ & -0.04699 & -1.41629 & -4.05767 \\
\hline $\mathrm{C}$ & -1.65260 & 2.30315 & 2.66434 \\
\hline $\mathrm{H}$ & -1.98145 & 3.30977 & 2.94227 \\
\hline $\mathrm{H}$ & -1.98550 & 1.61826 & 3.44808 \\
\hline $\mathrm{H}$ & -0.56048 & 2.29483 & 2.63890 \\
\hline $\mathrm{C}$ & -2.63246 & -2.36411 & 1.13311 \\
\hline $\mathrm{H}$ & -3.19642 & -3.02800 & 1.79635 \\
\hline $\mathrm{H}$ & -3.05019 & -2.48202 & 0.12741 \\
\hline $\mathrm{H}$ & -1.59653 & -2.71781 & 1.11802 \\
\hline $\mathrm{C}$ & 1.98112 & 2.20970 & -2.46740 \\
\hline $\mathrm{H}$ & 2.89005 & 2.58952 & -2.94486 \\
\hline $\mathrm{H}$ & 1.13020 & 2.57380 & -3.05242 \\
\hline $\mathrm{H}$ & 1.91659 & 2.66486 & -1.47526 \\
\hline C & 1.58858 & -2.76597 & -1.27112 \\
\hline $\mathrm{H}$ & 1.47056 & -3.79548 & -1.62572 \\
\hline $\mathrm{H}$ & 2.62182 & -2.46474 & -1.45767 \\
\hline $\mathrm{H}$ & 1.42208 & -2.76197 & -0.18922 \\
\hline $\mathrm{O}$ & -4.30672 & 0.89681 & 2.20321 \\
\hline $\mathrm{O}$ & 2.01975 & -1.30684 & -3.92681 \\
\hline
\end{tabular}

$4 a-M O L H-A$ 
Supporting Information for Donoghue et al.: Ligand and Substrate Effects on the Mechanisms of the Rhodium...

$\begin{array}{ll}\text { SCF Energy } & =-1833.622816 \text { hartree } \\ \text { Total Enthalpy } & =-1833.084111 \text { hartree }\end{array}$

\begin{tabular}{|c|c|c|c|}
\hline $\mathrm{Rh}$ & 0.12121 & 1.03515 & 0.97193 \\
\hline 0 & 0.44122 & 0.79369 & 3.13766 \\
\hline C & 1.65526 & 0.78291 & 3.42757 \\
\hline $\mathrm{N}$ & 2.60467 & 0.97725 & 2.50995 \\
\hline C & 2.21781 & 1.45969 & 1.18879 \\
\hline C & 1.49881 & 2.69824 & 1.12699 \\
\hline C & 3.09180 & 0.99711 & 0.15119 \\
\hline $\mathrm{N}$ & 3.80271 & 0.59950 & -0.68066 \\
\hline $\mathrm{H}$ & 1.35426 & 3.24023 & 2.05718 \\
\hline $\mathrm{H}$ & 1.56077 & 3.31414 & 0.24209 \\
\hline $\mathrm{H}$ & 3.58256 & 0.99539 & 2.78155 \\
\hline $\mathrm{H}$ & 1.98550 & 0.60412 & 4.45650 \\
\hline $\mathrm{P}$ & -0.15822 & 0.95597 & -1.35553 \\
\hline P & -0.61525 & -1.28086 & 0.93255 \\
\hline $\mathrm{H}$ & -1.70683 & 1.54253 & 1.31133 \\
\hline $\mathrm{H}$ & -1.31872 & 2.23107 & 1.23047 \\
\hline C & -1.71105 & 1.80842 & -1.98563 \\
\hline C & 1.10735 & 1.58745 & -2.60183 \\
\hline C & -2.12847 & -1.77715 & 1.92462 \\
\hline C & 0.61732 & -2.58615 & 1.47597 \\
\hline $\mathrm{H}$ & -1.58353 & 2.85179 & -1.67066 \\
\hline $\mathrm{H}$ & 0.91505 & -2.27180 & 2.48399 \\
\hline $\mathrm{H}$ & -2.98179 & -1.31941 & 1.40526 \\
\hline $\mathrm{H}$ & 1.96520 & 0.90902 & -2.53167 \\
\hline C & -1.06984 & -1.70638 & -0.82692 \\
\hline $\mathrm{H}$ & -0.90303 & -2.76823 & -1.03277 \\
\hline $\mathrm{H}$ & -2.14621 & -1.53299 & -0.92495 \\
\hline C & -0.29113 & -0.84938 & -1.83279 \\
\hline $\mathrm{H}$ & -0.73103 & -0.93985 & -2.83056 \\
\hline $\mathrm{H}$ & 0.74413 & -1.19996 & -1.90999 \\
\hline C & 0.50304 & 1.46410 & -4.02129 \\
\hline $\mathrm{H}$ & 1.24778 & 1.81732 & -4.74122 \\
\hline $\mathrm{H}$ & 0.26533 & 0.42135 & -4.27959 \\
\hline C & -1.78036 & 1.79066 & -3.53519 \\
\hline $\mathrm{H}$ & -2.61270 & 2.43136 & -3.84379 \\
\hline $\mathrm{H}$ & -2.00606 & 0.76855 & -3.88397 \\
\hline C & -0.04952 & -3.97361 & 1.60312 \\
\hline $\mathrm{H}$ & 0.66923 & -4.66856 & 2.04993 \\
\hline $\mathrm{H}$ & -0.30285 & -4.36374 & 0.60252 \\
\hline C & -2.30435 & -3.31031 & 1.89195 \\
\hline $\mathrm{H}$ & -3.16352 & -3.58681 & 2.51192 \\
\hline $\mathrm{H}$ & -2.50392 & -3.66465 & 0.86779 \\
\hline C & 1.58929 & 3.03037 & -2.36895 \\
\hline $\mathrm{H}$ & 2.10278 & 3.38335 & -3.26908 \\
\hline $\mathrm{H}$ & 0.76828 & 3.72668 & -2.17938 \\
\hline $\mathrm{H}$ & 2.31118 & 3.08834 & -1.55474 \\
\hline C & -3.03362 & 1.26968 & -1.42196 \\
\hline $\mathrm{H}$ & -3.85866 & 1.91161 & -1.74783 \\
\hline $\mathrm{H}$ & -3.24671 & 0.26084 & -1.79039 \\
\hline $\mathrm{H}$ & -3.05289 & 1.24740 & -0.33085 \\
\hline C & 1.85993 & -2.65811 & 0.57918 \\
\hline $\mathrm{H}$ & 2.58561 & -3.36252 & 0.99958 \\
\hline $\mathrm{H}$ & 1.61311 & -3.01656 & -0.42634 \\
\hline $\mathrm{H}$ & 2.35771 & -1.69062 & 0.47915 \\
\hline
\end{tabular}


Supporting Information for Donoghue et al.: Ligand and Substrate Effects on the Mechanisms of the Rhodium...

$\begin{array}{rrrr}\mathrm{C} & -2.07826 & -1.25628 & 3.37040 \\ \mathrm{H} & -3.02325 & -1.48788 & 3.87355 \\ \mathrm{H} & -1.27906 & -1.74358 & 3.93509 \\ \mathrm{H} & -1.92106 & -0.17692 & 3.41725 \\ \mathrm{O} & -0.63356 & 2.29058 & -4.18426 \\ \mathrm{O} & -1.18797 & -3.98916 & 2.44099\end{array}$

$4 a-M O L H-C$

SCF Energy $\quad=-1833.629851$ hartree

Total Enthalpy $=-1833.091390$ hartree

$\begin{array}{rrrr}\mathrm{Rh} & 0.65788 & -0.38679 & 0.94094 \\ \mathrm{O} & 0.54794 & -0.68725 & 3.11408 \\ \mathrm{C} & 1.68069 & -0.73623 & 3.64411 \\ \mathrm{~N} & 2.80146 & -0.52209 & 2.95461 \\ \mathrm{C} & 2.72045 & -0.06467 & 1.57678 \\ \mathrm{C} & 2.02745 & 1.17263 & 1.32337 \\ \mathrm{C} & 3.79579 & -0.50826 & 0.74667 \\ \mathrm{~N} & 4.67494 & -0.85524 & 0.06601 \\ \mathrm{H} & 1.72378 & 1.76443 & 2.18319 \\ \mathrm{H} & 2.33107 & 1.75590 & 0.46393 \\ \mathrm{H} & 3.70063 & -0.56160 & 3.42190 \\ \mathrm{H} & 1.78974 & -0.96335 & 4.70992 \\ \mathrm{P} & 0.67321 & -0.22588 & -1.37709 \\ \mathrm{P} & -1.64458 & 0.23324 & 0.76056 \\ \mathrm{H} & -0.04566 & -2.28291 & 0.85248 \\ \mathrm{H} & 0.72702 & -2.35591 & 0.88962 \\ \mathrm{C} & 1.88324 & 0.86575 & -2.29948 \\ \mathrm{C} & 0.92540 & -1.88570 & -2.22229 \\ \mathrm{C} & -2.08329 & 2.03479 & 1.04327 \\ \mathrm{C} & -2.88309 & -0.61349 & 1.87713 \\ \mathrm{H} & 2.86394 & 0.60216 & -1.88592 \\ \mathrm{H} & -2.49059 & -0.42825 & 2.88480 \\ \mathrm{H} & 0.01845 & -2.46815 & -2.00995 \\ \mathrm{H} & -1.66701 & 2.58918 & 0.19194 \\ \mathrm{C} & -1.01859 & 0.35122 & -1.94265 \\ \mathrm{H} & -0.98453 & 1.44535 & -1.96833 \\ \mathrm{H} & -1.20645 & 0.01912 & -2.96815 \\ \mathrm{C} & -2.13929 & -0.11698 & -1.00205 \\ \mathrm{H} & -2.29011 & -1.19856 & -1.08860 \\ \mathrm{H} & -3.08680 & 0.36157 & -1.26813 \\ \mathrm{C} & -3.61774 & 2.21231 & 1.03977 \\ \mathrm{H} & -3.85565 & 3.25937 & 1.25461 \\ \mathrm{H} & -4.04418 & 1.97266 & 0.05254 \\ \mathrm{C} & -4.27144 & 0.05892 & 1.78456 \\ \mathrm{H} & -4.92576 & -0.38417 & 2.54226 \\ \mathrm{H} & -4.72411 & -0.13965 & 0.79834 \\ \mathrm{C} & 1.89869 & 0.51629 & -3.81160 \\ \mathrm{H} & 2.72100 & 1.06634 & -4.27995 \\ \mathrm{H} & 0.96089 & 0.86027 & -4.27954 \\ \mathrm{C} & 1.02737 & -1.67698 & -3.75136 \\ \mathrm{H} & 1.21246 & -2.64629 & -4.22508 \\ \mathrm{H} & 0.09066 & -1.27782 & -4.17144 \\ \mathrm{C} & -1.47050 & 2.57322 & 2.34607 \\ \mathrm{H} & -1.67946 & 3.64420 & 2.43595 \\ \mathrm{H} & -1.90453 & 2.08047 & 3.21962 \\ & & & \end{array}$


Supporting Information for Donoghue et al.: Ligand and Substrate Effects on the Mechanisms of the Rhodium...

$\begin{array}{rrrr}\mathrm{H} & -0.38806 & 2.43614 & 2.37078 \\ \mathrm{C} & -2.98756 & -2.12913 & 1.66191 \\ \mathrm{H} & -3.68319 & -2.55801 & 2.39094 \\ \mathrm{H} & -3.36694 & -2.38172 & 0.66605 \\ \mathrm{H} & -2.02588 & -2.62909 & 1.80359 \\ \mathrm{C} & 1.63565 & 2.37514 & -2.13605 \\ \mathrm{H} & 2.49691 & 2.93202 & -2.51873 \\ \mathrm{H} & 0.76147 & 2.70193 & -2.70886 \\ \mathrm{H} & 1.48120 & 2.67930 & -1.09836 \\ \mathrm{C} & 2.16256 & -2.64749 & -1.71752 \\ \mathrm{H} & 2.19581 & -3.63116 & -2.19785 \\ \mathrm{H} & 3.08495 & -2.12298 & -1.97324 \\ \mathrm{H} & 2.15249 & -2.80578 & -0.63800 \\ \mathrm{O} & -4.25011 & 1.44871 & 2.04979 \\ \mathrm{O} & 2.11880 & -0.84744 & -4.09899\end{array}$

$4 a-M O L H-t s-A$

SCF Energy $=-1833.611256$ hartree
Total Enthalpy $=-1833.074211$ hartree
Negative Frequencies: $-849.19 \mathrm{~cm}^{-1}$

$\begin{array}{rrrr}\mathrm{Rh} & 0.04428 & 0.67066 & -1.01566 \\ \mathrm{O} & -1.66374 & 1.62545 & -2.02006 \\ \mathrm{C} & -1.97781 & 2.73691 & -1.54953 \\ \mathrm{~N} & -1.28621 & 3.32564 & -0.56953 \\ \mathrm{C} & -0.00729 & 2.78172 & -0.15395 \\ \mathrm{C} & 1.00523 & 2.64511 & -1.13758 \\ \mathrm{C} & 0.26056 & 2.94839 & 1.24005 \\ \mathrm{~N} & 0.45321 & 3.10668 & 2.37789 \\ \mathrm{H} & 0.82050 & 3.05608 & -2.12422 \\ \mathrm{H} & 2.04278 & 2.59861 & -0.83716 \\ \mathrm{H} & -1.57110 & 4.23758 & -0.22901 \\ \mathrm{H} & -2.85478 & 3.27482 & -1.92530 \\ \mathrm{P} & 1.72960 & -0.57648 & 0.00063 \\ \mathrm{P} & -1.53035 & -0.72151 & 0.31723 \\ \mathrm{H} & 0.06847 & -0.51047 & -2.10168 \\ \mathrm{H} & 0.79323 & 0.41410 & -2.40797 \\ \mathrm{C} & 3.11879 & -1.19043 & -1.09290 \\ \mathrm{C} & 2.70814 & 0.06662 & 1.47210 \\ \mathrm{C} & -2.47065 & -1.95640 & -0.74176 \\ \mathrm{C} & -2.89226 & -0.09769 & 1.44651 \\ \mathrm{H} & 3.60123 & -0.27526 & -1.45745 \\ \mathrm{H} & -3.47553 & 0.58029 & 0.81033 \\ \mathrm{H} & -1.70843 & -2.63280 & -1.15143 \\ \mathrm{H} & 1.99538 & 0.19006 & 2.29688 \\ \mathrm{C} & 0.87324 & -2.10936 & 0.63430 \\ \mathrm{H} & 0.65848 & -2.72924 & -0.24275 \\ \mathrm{H} & 1.54891 & -2.68671 & 1.27120 \\ \mathrm{C} & -0.42314 & -1.77522 & 1.38480 \\ \mathrm{H} & -0.20436 & -1.20935 & 2.29659 \\ \mathrm{H} & -0.92496 & -2.69690 & 1.69485 \\ \mathrm{C} & -3.83241 & -1.24569 & 1.87896 \\ \mathrm{H} & -3.30329 & -1.92378 & 2.56985 \\ \mathrm{H} & -4.67847 & -0.81628 & 2.42569 \\ \mathrm{C} & -3.44332 & -2.78399 & 0.12361 \\ \mathrm{H} & -4.02208 & -3.44823 & -0.52658\end{array}$


Supporting Information for Donoghue et al.: Ligand and Substrate Effects on the Mechanisms of the Rhodium...

$\begin{array}{rrrr}\mathrm{H} & -2.89998 & -3.41800 & 0.84279 \\ \mathrm{C} & 3.75319 & -1.00712 & 1.87108 \\ \mathrm{H} & 4.32293 & -0.62760 & 2.72503 \\ \mathrm{H} & 3.27933 & -1.94863 & 2.18639 \\ \mathrm{C} & 4.16644 & -1.96762 & -0.25287 \\ \mathrm{H} & 5.01747 & -2.19873 & -0.90076 \\ \mathrm{H} & 3.74036 & -2.92634 & 0.08625 \\ \mathrm{C} & 2.67755 & -2.03326 & -2.29607 \\ \mathrm{H} & 2.00021 & -1.48845 & -2.95661 \\ \mathrm{H} & 3.55676 & -2.30936 & -2.88723 \\ \mathrm{H} & 2.18742 & -2.96393 & -1.99206 \\ \mathrm{C} & -2.37910 & 0.67338 & 2.66969 \\ \mathrm{H} & -1.85783 & 0.01676 & 3.37415 \\ \mathrm{H} & -1.69814 & 1.48374 & 2.40203 \\ \mathrm{H} & -3.22361 & 1.11457 & 3.20965 \\ \mathrm{C} & 3.42490 & 1.40391 & 1.21951 \\ \mathrm{H} & 2.72747 & 2.23168 & 1.11032 \\ \mathrm{H} & 4.05354 & 1.63577 & 2.08506 \\ \mathrm{H} & 4.08498 & 1.35661 & 0.35018 \\ \mathrm{C} & -3.21216 & -1.27337 & -1.90302 \\ \mathrm{H} & -2.55064 & -0.64858 & -2.50537 \\ \mathrm{H} & -3.65170 & -2.03681 & -2.55354 \\ \mathrm{H} & -4.03116 & -0.65207 & -1.53037 \\ \mathrm{O} & -4.38671 & -1.97227 & 0.80020 \\ \mathrm{O} & 4.69097 & -1.24122 & 0.83945\end{array}$

4a-MOLH-ts-C

SCF Energy $=-1833.613949$ hartree
Total Enthalpy $=-1833.077234$ hartree
Negative Frequencies: $-784.14 \mathrm{~cm}^{-1}$

$\begin{array}{rrrr}\mathrm{Rh} & -0.06927 & 0.92196 & -0.46936 \\ \mathrm{O} & 1.17862 & 2.64039 & -1.06198 \\ \mathrm{C} & 0.63850 & 3.72881 & -0.78075 \\ \mathrm{~N} & -0.48608 & 3.81119 & -0.06231 \\ \mathrm{C} & -1.02700 & 2.63021 & 0.58403 \\ \mathrm{C} & -0.22124 & 1.94267 & 1.52296 \\ \mathrm{C} & -2.45929 & 2.59025 & 0.64904 \\ \mathrm{~N} & -3.61938 & 2.55642 & 0.73357 \\ \mathrm{H} & 0.73210 & 2.37508 & 1.80274 \\ \mathrm{H} & -0.70686 & 1.33120 & 2.27042 \\ \mathrm{H} & -0.89877 & 4.71648 & 0.13372 \\ \mathrm{H} & 1.07277 & 4.67445 & -1.12247 \\ \mathrm{P} & -1.42053 & -0.92425 & -0.13165 \\ \mathrm{P} & 1.81129 & -0.54129 & 0.07771 \\ \mathrm{H} & 0.02118 & 0.35801 & -1.96348 \\ \mathrm{H} & -0.94946 & 1.23517 & -1.76982 \\ \mathrm{C} & -2.41113 & -1.09619 & 1.44713 \\ \mathrm{C} & -2.71266 & -1.23279 & -1.44923 \\ \mathrm{C} & 2.97550 & -0.18438 & 1.50193 \\ \mathrm{C} & 3.05274 & -0.84103 & -1.29409 \\ \mathrm{H} & -2.96719 & -0.15513 & 1.53111 \\ \mathrm{H} & 3.44192 & 0.15870 & -1.52408 \\ \mathrm{H} & -2.14638 & -1.51110 & -2.34795 \\ \mathrm{H} & 2.37684 & -0.28755 & 2.41648 \\ \mathrm{C} & -0.29762 & -2.41607 & -0.21886\end{array}$


Supporting Information for Donoghue et al.: Ligand and Substrate Effects on the Mechanisms of the Rhodium...

\begin{tabular}{|c|c|c|c|}
\hline $\mathrm{H}$ & -0.80847 & -3.29134 & 0.19587 \\
\hline $\mathrm{H}$ & -0.14804 & -2.61096 & -1.28625 \\
\hline $\mathrm{C}$ & 1.05715 & -2.20573 & 0.47932 \\
\hline $\mathrm{H}$ & 1.73983 & -3.01864 & 0.21341 \\
\hline $\mathrm{H}$ & 0.93665 & -2.23929 & 1.56687 \\
\hline $\mathrm{C}$ & 2.44493 & -1.44177 & -2.56694 \\
\hline $\mathrm{H}$ & 2.05895 & -2.45350 & -2.39770 \\
\hline $\mathrm{H}$ & 1.63449 & -0.82263 & -2.95992 \\
\hline $\mathrm{H}$ & 3.21066 & -1.51478 & -3.34615 \\
\hline $\mathrm{C}$ & 4.23311 & -1.70656 & -0.79429 \\
\hline $\mathrm{H}$ & 4.98957 & -1.75135 & -1.58434 \\
\hline $\mathrm{H}$ & 3.89232 & -2.73963 & -0.60892 \\
\hline $\mathrm{C}$ & 4.11117 & -1.23100 & 1.52864 \\
\hline $\mathrm{H}$ & 4.79508 & -0.99919 & 2.35188 \\
\hline $\mathrm{H}$ & 3.71478 & -2.24457 & 1.70045 \\
\hline $\mathrm{C}$ & 3.55949 & 1.23716 & 1.41902 \\
\hline $\mathrm{H}$ & 2.79341 & 1.99663 & 1.24886 \\
\hline $\mathrm{H}$ & 4.07765 & 1.47721 & 2.35321 \\
\hline $\mathrm{H}$ & 4.29122 & 1.30644 & 0.61063 \\
\hline O & 4.89184 & -1.19344 & 0.34774 \\
\hline $\mathrm{C}$ & -1.56819 & -1.32372 & 2.71233 \\
\hline $\mathrm{H}$ & -1.12531 & -2.32545 & 2.72094 \\
\hline $\mathrm{H}$ & -0.75795 & -0.60044 & 2.82652 \\
\hline $\mathrm{H}$ & -2.20592 & -1.24612 & 3.59896 \\
\hline $\mathrm{C}$ & -3.44669 & -2.24791 & 1.32201 \\
\hline $\mathrm{H}$ & -4.10793 & -2.20121 & 2.19316 \\
\hline $\mathrm{H}$ & -2.92370 & -3.21902 & 1.35620 \\
\hline $\mathrm{C}$ & -3.59156 & -2.43240 & -1.02578 \\
\hline $\mathrm{H}$ & -4.36006 & -2.59148 & -1.78823 \\
\hline $\mathrm{H}$ & -3.00113 & -3.35929 & -0.94928 \\
\hline $\mathrm{C}$ & -3.58872 & -0.00726 & -1.75456 \\
\hline $\mathrm{H}$ & -2.99700 & 0.84842 & -2.08602 \\
\hline $\mathrm{H}$ & -4.28679 & -0.26280 & -2.55871 \\
\hline $\mathrm{H}$ & -4.17852 & 0.29553 & -0.88721 \\
\hline O & -4.27746 & -2.17879 & 0.1863 \\
\hline
\end{tabular}

4a-DIHY-A

SCF Energy $=-1833.617655$ hartree Total Enthalpy $=-1833.077910$ hartree

$\begin{array}{rrrr}\mathrm{Rh} & -0.15957 & 0.99864 & 0.99880 \\ \mathrm{O} & -0.40895 & 0.78946 & 3.20208 \\ \mathrm{C} & 0.63482 & 0.74444 & 3.87311 \\ \mathrm{~N} & 1.85377 & 0.96244 & 3.35088 \\ \mathrm{C} & 2.00544 & 1.46156 & 2.00920 \\ \mathrm{C} & 1.35545 & 2.63371 & 1.63822 \\ \mathrm{C} & 3.11836 & 0.93939 & 1.28026 \\ \mathrm{~N} & 4.05514 & 0.54550 & 0.71188 \\ \mathrm{H} & 0.83324 & 3.20929 & 2.39281 \\ \mathrm{H} & 1.64933 & 3.15892 & 0.74079 \\ \mathrm{H} & 2.66996 & 0.90695 & 3.94942 \\ \mathrm{H} & 0.60806 & 0.50769 & 4.94311 \\ \mathrm{P} & -0.22405 & 1.11986 & -1.30092 \\ \mathrm{P} & -0.16209 & -1.48993 & 0.69560 \\ \mathrm{H} & -1.66951 & 0.76310 & 0.74906 \\ \mathrm{H} & -0.81217 & 2.43129 & 0.97307\end{array}$


Supporting Information for Donoghue et al.: Ligand and Substrate Effects on the Mechanisms of the Rhodium...

\begin{tabular}{|c|c|c|c|}
\hline $\mathrm{C}$ & -1.34851 & 2.41603 & -2.03588 \\
\hline C & 1.33428 & 1.35756 & -2.32400 \\
\hline C & -1.82239 & -2.20704 & 1.21162 \\
\hline C & 1.02498 & -2.78767 & 1.35863 \\
\hline $\mathrm{H}$ & -0.93805 & 3.36190 & -1.66118 \\
\hline $\mathrm{H}$ & 1.07388 & -2.57760 & 2.43491 \\
\hline $\mathrm{H}$ & -2.56071 & -1.75077 & 0.53898 \\
\hline $\mathrm{H}$ & 1.92616 & 0.44071 & -2.20941 \\
\hline C & -0.87767 & -0.53737 & -1.85329 \\
\hline $\mathrm{H}$ & -1.94349 & -0.55356 & -1.60350 \\
\hline $\mathrm{H}$ & -0.80385 & -0.63405 & -2.94022 \\
\hline C & -0.14237 & -1.69605 & -1.16097 \\
\hline $\mathrm{H}$ & 0.90562 & -1.72621 & -1.47710 \\
\hline $\mathrm{H}$ & -0.58886 & -2.65066 & -1.45546 \\
\hline C & 0.92546 & 1.49253 & -3.81393 \\
\hline $\mathrm{H}$ & 1.83282 & 1.65377 & -4.40463 \\
\hline $\mathrm{H}$ & 0.44901 & 0.57394 & -4.18907 \\
\hline C & -1.23013 & 2.43921 & -3.57826 \\
\hline $\mathrm{H}$ & -1.80090 & 3.29303 & -3.95590 \\
\hline $\mathrm{H}$ & -1.67620 & 1.52722 & -4.00856 \\
\hline C & 0.45803 & -4.21584 & 1.19091 \\
\hline $\mathrm{H}$ & 1.11478 & -4.91370 & 1.72092 \\
\hline $\mathrm{H}$ & 0.46851 & -4.49857 & 0.12451 \\
\hline C & -1.84634 & -3.73408 & 0.99818 \\
\hline $\mathrm{H}$ & -2.79970 & -4.12924 & 1.36419 \\
\hline $\mathrm{H}$ & -1.77030 & -3.99338 & -0.07013 \\
\hline C & 2.19036 & 2.56138 & -1.89913 \\
\hline $\mathrm{H}$ & 2.99724 & 2.70111 & -2.62580 \\
\hline $\mathrm{H}$ & 1.61357 & 3.48992 & -1.88000 \\
\hline $\mathrm{H}$ & 2.66473 & 2.39955 & -0.93155 \\
\hline C & -2.81229 & 2.30117 & -1.59400 \\
\hline $\mathrm{H}$ & -3.38536 & 3.13962 & -2.00310 \\
\hline $\mathrm{H}$ & -3.28078 & 1.37995 & -1.95626 \\
\hline $\mathrm{H}$ & -2.90840 & 2.33278 & -0.50629 \\
\hline C & 2.43649 & -2.70305 & 0.76180 \\
\hline $\mathrm{H}$ & 3.09854 & -3.40290 & 1.28265 \\
\hline $\mathrm{H}$ & 2.44495 & -2.98479 & -0.29639 \\
\hline $\mathrm{H}$ & 2.87556 & -1.70840 & 0.84898 \\
\hline C & -2.18418 & -1.85434 & 2.66376 \\
\hline $\mathrm{H}$ & -3.18961 & -2.22622 & 2.88766 \\
\hline $\mathrm{H}$ & -1.49696 & -2.33682 & 3.36482 \\
\hline $\mathrm{H}$ & -2.16814 & -0.77874 & 2.84358 \\
\hline 0 & 0.09486 & 2.61264 & -4.04386 \\
\hline 0 & -0.83339 & -4.39480 & 1.73620 \\
\hline
\end{tabular}

$4 a-D I H Y-C$

SCF Energy $\quad=-1833.618015$ hartree Total Enthalpy $=-1833.078712$ hartree

$\begin{array}{rrrr}\text { Rh } & 0.62389 & -0.45622 & 0.94392 \\ \mathrm{O} & 0.58454 & -0.64752 & 3.15910 \\ \mathrm{C} & 1.71329 & -0.61760 & 3.67317 \\ \mathrm{~N} & 2.82083 & -0.26631 & 2.99543 \\ \mathrm{C} & 2.74551 & 0.25609 & 1.65292 \\ \mathrm{C} & 2.02304 & 1.41055 & 1.39916 \\ \mathrm{C} & 3.76385 & -0.21674 & 0.75702\end{array}$


Supporting Information for Donoghue et al.: Ligand and Substrate Effects on the Mechanisms of the Rhodium...

\begin{tabular}{|c|c|c|c|}
\hline $\mathrm{N}$ & 4.61499 & -0.56173 & 0.04383 \\
\hline $\mathrm{H}$ & 1.55870 & 1.94023 & 2.21956 \\
\hline $\mathrm{H}$ & 2.16256 & 1.94532 & 0.47095 \\
\hline $\mathrm{H}$ & 3.72567 & -0.30912 & 3.45023 \\
\hline $\mathrm{H}$ & 1.86395 & -0.88417 & 4.72547 \\
\hline $\mathrm{P}$ & 0.55714 & -0.71796 & -1.34214 \\
\hline $\mathrm{P}$ & -1.28160 & 1.10559 & 0.72088 \\
\hline $\mathrm{H}$ & -0.47911 & -1.53829 & 0.81342 \\
\hline $\mathrm{H}$ & 1.43948 & -1.80577 & 0.92884 \\
\hline $\mathrm{C}$ & 1.92649 & -0.05913 & -2.43951 \\
\hline $\mathrm{C}$ & 0.40878 & -2.48245 & -1.95115 \\
\hline $\mathrm{C}$ & -1.30758 & 2.84867 & 1.41417 \\
\hline $\mathrm{C}$ & -2.95461 & 0.50905 & 1.32300 \\
\hline $\mathrm{H}$ & 2.84737 & -0.48944 & -2.02994 \\
\hline $\mathrm{H}$ & -2.81405 & 0.38146 & 2.40370 \\
\hline $\mathrm{H}$ & -0.57757 & -2.82460 & -1.61182 \\
\hline $\mathrm{H}$ & -0.51042 & 3.39608 & 0.89380 \\
\hline $\mathrm{C}$ & -1.02594 & 0.09132 & -1.92783 \\
\hline $\mathrm{H}$ & -0.94314 & 0.33693 & -2.99146 \\
\hline $\mathrm{H}$ & -1.80098 & -0.67746 & -1.84262 \\
\hline $\mathrm{C}$ & -1.42989 & 1.34229 & -1.12886 \\
\hline $\mathrm{H}$ & -2.44431 & 1.64207 & -1.40944 \\
\hline $\mathrm{H}$ & -0.77328 & 2.18289 & -1.38063 \\
\hline $\mathrm{C}$ & -2.65994 & 3.52653 & 1.10161 \\
\hline $\mathrm{H}$ & -2.66528 & 4.53527 & 1.52782 \\
\hline $\mathrm{H}$ & -2.81629 & 3.62409 & 0.01573 \\
\hline $\mathrm{C}$ & -4.03934 & 1.59115 & 1.11222 \\
\hline $\mathrm{H}$ & -4.96715 & 1.24890 & 1.58191 \\
\hline $\mathrm{H}$ & -4.24533 & 1.71545 & 0.03547 \\
\hline $\mathrm{C}$ & 1.76169 & -0.58140 & -3.89209 \\
\hline $\mathrm{H}$ & 2.65883 & -0.29857 & -4.45199 \\
\hline $\mathrm{H}$ & 0.90277 & -0.08313 & -4.37394 \\
\hline $\mathrm{C}$ & 0.42878 & -2.48924 & -3.49657 \\
\hline $\mathrm{H}$ & 0.35466 & -3.52344 & -3.84634 \\
\hline $\mathrm{H}$ & -0.42730 & -1.93546 & -3.91423 \\
\hline $\mathrm{C}$ & -1.05198 & 2.86343 & 2.93337 \\
\hline $\mathrm{H}$ & -0.82337 & 3.88257 & 3.26179 \\
\hline $\mathrm{H}$ & -1.94524 & 2.53772 & 3.47116 \\
\hline $\mathrm{H}$ & -0.22664 & 2.21421 & 3.23645 \\
\hline $\mathrm{C}$ & -3.40915 & -0.82798 & 0.72551 \\
\hline $\mathrm{H}$ & -4.34165 & -1.14871 & 1.20146 \\
\hline $\mathrm{H}$ & -3.60707 & -0.74888 & -0.34923 \\
\hline $\mathrm{H}$ & -2.67004 & -1.61698 & 0.88604 \\
\hline $\mathrm{C}$ & 2.04820 & 1.47057 & -2.46956 \\
\hline $\mathrm{H}$ & 2.87792 & 1.75874 & -3.12362 \\
\hline $\mathrm{H}$ & 1.14443 & 1.94713 & -2.86428 \\
\hline $\mathrm{H}$ & 2.25754 & 1.89647 & -1.48707 \\
\hline $\mathrm{C}$ & 1.49102 & -3.41861 & -1.39306 \\
\hline $\mathrm{H}$ & 1.34030 & -4.42222 & -1.80411 \\
\hline $\mathrm{H}$ & 2.49376 & -3.09441 & -1.68205 \\
\hline $\mathrm{H}$ & 1.44314 & -3.48978 & -0.30522 \\
\hline $\mathrm{O}$ & 1.64673 & -1.98121 & -4.00897 \\
\hline $\mathrm{O}$ & -3.74316 & 2.84188 & 1.70455 \\
\hline
\end{tabular}

$4 a-D I H Y-t s-A$

$\mathrm{SCF}$ Energy $=-1833.614025$ hartree 
Supporting Information for Donoghue et al.: Ligand and Substrate Effects on the Mechanisms of the Rhodium...

Total Enthalpy $=-1833.075959$ hartree Negative Frequencies: $-633.64 \mathrm{~cm}^{-1}$

\begin{tabular}{|c|c|c|c|}
\hline $\mathrm{Rh}$ & 0.03179 & 0.63039 & -0.93491 \\
\hline $\mathrm{O}$ & -1.67164 & 1.51727 & -2.05211 \\
\hline C & -2.06152 & 2.62960 & -1.64976 \\
\hline $\mathrm{N}$ & -1.41807 & 3.32475 & -0.70264 \\
\hline C & -0.13566 & 2.88157 & -0.21584 \\
\hline C & 0.92466 & 2.75416 & -1.15249 \\
\hline C & 0.08860 & 3.08297 & 1.17641 \\
\hline $\mathrm{N}$ & 0.25551 & 3.27844 & 2.31349 \\
\hline $\mathrm{H}$ & 0.76901 & 3.18319 & -2.13807 \\
\hline $\mathrm{H}$ & 1.94366 & 2.78664 & -0.78821 \\
\hline $\mathrm{H}$ & -1.78357 & 4.22123 & -0.40091 \\
\hline $\mathrm{H}$ & -2.96625 & 3.09085 & -2.06101 \\
\hline $\mathrm{P}$ & 1.74064 & -0.59880 & 0.00955 \\
\hline $\mathrm{P}$ & -1.48387 & -0.77700 & 0.33167 \\
\hline $\mathrm{H}$ & 0.08255 & -0.61007 & -1.87808 \\
\hline $\mathrm{H}$ & 1.10758 & 1.25924 & -1.96576 \\
\hline C & 3.13222 & -1.09989 & -1.12969 \\
\hline $\mathrm{C}$ & 2.70395 & 0.02807 & 1.49576 \\
\hline C & -2.43414 & -1.98795 & -0.74225 \\
\hline C & -2.82108 & -0.14914 & 1.48268 \\
\hline $\mathrm{H}$ & 3.55817 & -0.14409 & -1.46029 \\
\hline $\mathrm{H}$ & -3.39428 & 0.55381 & 0.86454 \\
\hline $\mathrm{H}$ & -1.67762 & -2.67049 & -1.15199 \\
\hline $\mathrm{H}$ & 2.00109 & 0.05146 & 2.33810 \\
\hline C & 0.93282 & -2.16658 & 0.61135 \\
\hline $\mathrm{H}$ & 0.73172 & -2.77867 & -0.27395 \\
\hline $\mathrm{H}$ & 1.61833 & -2.73537 & 1.24564 \\
\hline C & -0.37041 & -1.85951 & 1.36491 \\
\hline $\mathrm{H}$ & -0.15678 & -1.32253 & 2.29500 \\
\hline $\mathrm{H}$ & -0.87499 & -2.78963 & 1.64345 \\
\hline C & -3.78098 & -1.28800 & 1.89752 \\
\hline $\mathrm{H}$ & -3.26317 & -1.98473 & 2.57840 \\
\hline $\mathrm{H}$ & -4.61950 & -0.85178 & 2.45039 \\
\hline C & -3.41435 & -2.80839 & 0.12348 \\
\hline $\mathrm{H}$ & -4.00206 & -3.46100 & -0.53024 \\
\hline $\mathrm{H}$ & -2.87760 & -3.45354 & 0.83756 \\
\hline C & 3.82773 & -0.98968 & 1.81912 \\
\hline $\mathrm{H}$ & 4.39828 & -0.61087 & 2.67306 \\
\hline $\mathrm{H}$ & 3.41923 & -1.97044 & 2.10701 \\
\hline C & 4.23713 & -1.85119 & -0.34809 \\
\hline $\mathrm{H}$ & 5.08343 & -2.01825 & -1.02139 \\
\hline $\mathrm{H}$ & 3.86953 & -2.84015 & -0.02743 \\
\hline C & 2.68747 & -1.89569 & -2.36222 \\
\hline $\mathrm{H}$ & 1.94134 & -1.35128 & -2.94558 \\
\hline $\mathrm{H}$ & 3.55027 & -2.07834 & -3.01095 \\
\hline $\mathrm{H}$ & 2.27000 & -2.87181 & -2.09354 \\
\hline C & -2.28032 & 0.58497 & 2.71708 \\
\hline $\mathrm{H}$ & -1.77375 & -0.09995 & 3.40539 \\
\hline $\mathrm{H}$ & -1.58144 & 1.38371 & 2.46117 \\
\hline $\mathrm{H}$ & -3.11025 & 1.03702 & 3.27039 \\
\hline C & 3.30363 & 1.43003 & 1.30400 \\
\hline $\mathrm{H}$ & 2.53242 & 2.19541 & 1.22975 \\
\hline $\mathrm{H}$ & 3.91310 & 1.68101 & 2.17830 \\
\hline $\mathrm{H}$ & 3.96201 & 1.47583 & 0.4324 \\
\hline
\end{tabular}


Supporting Information for Donoghue et al.: Ligand and Substrate Effects on the Mechanisms of the Rhodium...

$\begin{array}{rrrr}\mathrm{C} & -3.16962 & -1.30048 & -1.90383 \\ \mathrm{H} & -2.49906 & -0.69642 & -2.51573 \\ \mathrm{H} & -3.62796 & -2.06325 & -2.54211 \\ \mathrm{H} & -3.97408 & -0.65964 & -1.53244 \\ \mathrm{O} & -4.34549 & -1.98889 & 0.80751 \\ \mathrm{O} & 4.74813 & -1.12796 & 0.75495\end{array}$

$4 a-D I H Y-t s-C$

$\mathrm{SCF}$ Energy $=-1833.595968$ hartree
Total Enthalpy $=-1833.059413$ hartree
Negative Frequencies: $-723.42 \mathrm{~cm}^{-1}$

\begin{tabular}{|c|c|c|c|}
\hline $\mathrm{Rh}$ & -0.04211 & 0.81384 & -0.40590 \\
\hline 0 & 1.15290 & 2.58656 & -1.10774 \\
\hline C & 0.57325 & 3.67550 & -0.97370 \\
\hline $\mathrm{N}$ & -0.59499 & 3.81549 & -0.32207 \\
\hline $\mathrm{C}$ & -1.17620 & 2.68298 & 0.38796 \\
\hline $\mathrm{C}$ & -0.38084 & 2.09845 & 1.43504 \\
\hline C & -2.60594 & 2.81826 & 0.60696 \\
\hline $\mathrm{N}$ & -3.74440 & 2.92004 & 0.81138 \\
\hline $\mathrm{H}$ & 0.52711 & 2.62123 & 1.70831 \\
\hline $\mathrm{H}$ & -0.90395 & 1.59646 & 2.23894 \\
\hline $\mathrm{H}$ & -1.03859 & 4.72317 & -0.24993 \\
\hline $\mathrm{H}$ & 0.99024 & 4.59562 & -1.39927 \\
\hline P & -1.35132 & -1.03462 & -0.14253 \\
\hline $\mathrm{P}$ & 1.79380 & -0.55064 & 0.10108 \\
\hline $\mathrm{H}$ & -0.04439 & 0.22118 & -1.88159 \\
\hline $\mathrm{H}$ & -1.35026 & 1.79273 & -0.79775 \\
\hline C & -2.35529 & -1.16957 & 1.43044 \\
\hline C & -2.64364 & -1.35100 & -1.45305 \\
\hline C & 2.91267 & -0.15419 & 1.54687 \\
\hline C & 3.04679 & -0.71928 & -1.27607 \\
\hline $\mathrm{H}$ & -2.92090 & -0.23024 & 1.47443 \\
\hline $\mathrm{H}$ & 3.35844 & 0.31278 & -1.47923 \\
\hline $\mathrm{H}$ & -2.08260 & -1.64893 & -2.34804 \\
\hline $\mathrm{H}$ & 2.31566 & -0.33731 & 2.44983 \\
\hline C & -0.22174 & -2.51743 & -0.21466 \\
\hline $\mathrm{H}$ & -0.71246 & -3.39269 & 0.22297 \\
\hline $\mathrm{H}$ & -0.08082 & -2.72631 & -1.28034 \\
\hline C & 1.13419 & -2.26185 & 0.46388 \\
\hline $\mathrm{H}$ & 1.85699 & -3.02631 & 0.16274 \\
\hline $\mathrm{H}$ & 1.03623 & -2.33042 & 1.55207 \\
\hline C & 2.48356 & -1.33265 & -2.56450 \\
\hline $\mathrm{H}$ & 2.16452 & -2.37081 & -2.41673 \\
\hline $\mathrm{H}$ & 1.63511 & -0.75963 & -2.94444 \\
\hline $\mathrm{H}$ & 3.25714 & -1.33923 & -3.33938 \\
\hline $\mathrm{C}$ & 4.28866 & -1.50680 & -0.79335 \\
\hline $\mathrm{H}$ & 5.04814 & -1.46954 & -1.58081 \\
\hline $\mathrm{H}$ & 4.02603 & -2.56764 & -0.64076 \\
\hline C & 4.12073 & -1.11887 & 1.54062 \\
\hline $\mathrm{H}$ & 4.78101 & -0.87016 & 2.37790 \\
\hline $\mathrm{H}$ & 3.79751 & -2.16418 & 1.67054 \\
\hline C & 3.39078 & 1.30679 & 1.52976 \\
\hline $\mathrm{H}$ & 2.56240 & 2.00948 & 1.42837 \\
\hline $\mathrm{H}$ & 3.91580 & 1.53071 & 2.46400 \\
\hline $\mathrm{H}$ & 4.09153 & 1.47807 & 0.70939 \\
\hline
\end{tabular}


Supporting Information for Donoghue et al.: Ligand and Substrate Effects on the Mechanisms of the Rhodium...

$\begin{array}{rrrr}\mathrm{O} & 4.90088 & -0.97963 & 0.36752 \\ \mathrm{C} & -1.50951 & -1.31854 & 2.70316 \\ \mathrm{H} & -1.02468 & -2.29974 & 2.74936 \\ \mathrm{H} & -0.73413 & -0.55235 & 2.77919 \\ \mathrm{H} & -2.14927 & -1.23339 & 3.58752 \\ \mathrm{C} & -3.38393 & -2.32528 & 1.33458 \\ \mathrm{H} & -4.04270 & -2.27138 & 2.20730 \\ \mathrm{H} & -2.85998 & -3.29564 & 1.37606 \\ \mathrm{C} & -3.54039 & -2.53179 & -1.01365 \\ \mathrm{H} & -4.31407 & -2.68844 & -1.77159 \\ \mathrm{H} & -2.96022 & -3.46502 & -0.93286 \\ \mathrm{C} & -3.48595 & -0.10732 & -1.77617 \\ \mathrm{H} & -2.86376 & 0.71130 & -2.14475 \\ \mathrm{H} & -4.20956 & -0.35611 & -2.55935 \\ \mathrm{H} & -4.05346 & 0.23689 & -0.90769 \\ \mathrm{O} & -4.21949 & -2.26401 & 0.19970\end{array}$

$4 a-A L H Y-A$

SCF Energy $\quad=-1833.655642$ hartree

Total Enthalpy $=-1833.112648$ hartree

$\begin{array}{rrrr}\mathrm{Rh} & 0.83527 & -0.11498 & 0.98555 \\ \mathrm{O} & 0.75862 & -0.32539 & 3.11654 \\ \mathrm{C} & 1.90676 & -0.34647 & 3.62921 \\ \mathrm{~N} & 3.00855 & -0.23126 & 2.90810 \\ \mathrm{C} & 2.95822 & -0.14782 & 1.42779 \\ \mathrm{C} & 3.91830 & 0.94988 & 0.96675 \\ \mathrm{C} & 3.26111 & -1.46345 & 0.88776 \\ \mathrm{~N} & 3.40154 & -2.52796 & 0.43079 \\ \mathrm{H} & 4.93447 & 0.78131 & 1.34960 \\ \mathrm{H} & 3.98764 & 0.98328 & -0.12009 \\ \mathrm{H} & 3.91028 & -0.27167 & 3.37552 \\ \mathrm{H} & 2.02033 & -0.46013 & 4.71210 \\ \mathrm{P} & 0.75398 & 0.09396 & -1.33308 \\ \mathrm{P} & -1.58191 & -0.02327 & 0.85357 \\ \mathrm{H} & 0.91614 & 1.39200 & 0.98149 \\ \mathrm{H} & 3.57401 & 1.92391 & 1.32251 \\ \mathrm{C} & 2.04834 & 1.07646 & -2.27525 \\ \mathrm{C} & 0.67025 & -1.52623 & -2.29031 \\ \mathrm{C} & -2.40278 & 1.31885 & 1.86473 \\ \mathrm{C} & -2.54550 & -1.52626 & 1.43262 \\ \mathrm{H} & 0.03846 & -1.28848 & -3.15679 \\ \mathrm{H} & -3.42716 & 1.38798 & 1.47602 \\ \mathrm{H} & 2.45038 & 1.80463 & -1.56153 \\ \mathrm{H} & -1.96639 & -2.40423 & 1.11989 \\ \mathrm{C} & -0.87033 & 0.94066 & -1.68946 \\ \mathrm{H} & -0.74347 & 1.98710 & -1.39084 \\ \mathrm{H} & -1.06045 & 0.93244 & -2.76721 \\ \mathrm{C} & -2.04030 & 0.30215 & -0.92574 \\ \mathrm{H} & -2.31203 & -0.65483 & -1.38121 \\ \mathrm{H} & -2.93003 & 0.93826 & -0.97919 \\ \mathrm{C} & 2.05628 & -1.94301 & -2.83354 \\ \mathrm{H} & 1.90842 & -2.76349 & -3.54279 \\ \mathrm{H} & 2.68914 & -2.31347 & -2.01510 \\ \mathrm{C} & 3.18629 & 0.12080 & -2.70572 \\ \mathrm{H} & 3.70520 & -0.30906 & -1.83674 \\ & & & \\ & & & \end{array}$


Supporting Information for Donoghue et al.: Ligand and Substrate Effects on the Mechanisms of the Rhodium...

$\begin{array}{rrrr}\mathrm{H} & 3.91913 & 0.69010 & -3.28657 \\ \mathrm{C} & -2.55222 & -1.49228 & 2.98016 \\ \mathrm{H} & -3.10414 & -2.36253 & 3.34894 \\ \mathrm{H} & -1.52724 & -1.54288 & 3.37530 \\ \mathrm{C} & -2.50202 & 0.85570 & 3.33568 \\ \mathrm{H} & -1.49653 & 0.76286 & 3.77240 \\ \mathrm{H} & -3.05160 & 1.61340 & 3.90339 \\ \mathrm{C} & 0.05053 & -2.68978 & -1.50123 \\ \mathrm{H} & 0.01232 & -3.58249 & -2.13525 \\ \mathrm{H} & 0.65430 & -2.94065 & -0.62334 \\ \mathrm{H} & -0.97257 & -2.48441 & -1.17727 \\ \mathrm{C} & 1.49119 & 1.82214 & -3.50160 \\ \mathrm{H} & 2.30837 & 2.37322 & -3.97927 \\ \mathrm{H} & 1.09269 & 1.12647 & -4.24447 \\ \mathrm{H} & 0.71874 & 2.54912 & -3.24113 \\ \mathrm{C} & -3.97376 & -1.62173 & 0.87076 \\ \mathrm{H} & -4.43897 & -2.54730 & 1.22712 \\ \mathrm{H} & -4.59428 & -0.79224 & 1.21785 \\ \mathrm{H} & -3.99901 & -1.64666 & -0.22199 \\ \mathrm{C} & -1.72498 & 2.68891 & 1.74771 \\ \mathrm{H} & -2.28831 & 3.43282 & 2.32096 \\ \mathrm{H} & -0.70378 & 2.66589 & 2.14072 \\ \mathrm{H} & -1.68406 & 3.04234 & 0.71260 \\ \mathrm{O} & 2.71498 & -0.91248 & -3.54900 \\ \mathrm{O} & -3.22699 & -0.35311 & 3.48902\end{array}$

\section{$4 a-A L H Y-C$}

$\begin{array}{ll}\text { SCF Energy } & =-1833.640197 \text { hartree } \\ \text { Total Enthalpy } & =-1833.097392 \text { hartree }\end{array}$

$\begin{array}{rrrr}\text { Rh } & 0.73773 & 0.10939 & 0.80727 \\ \mathrm{O} & 0.66163 & 0.31209 & 2.97246 \\ \mathrm{C} & 1.63055 & 0.45093 & 3.74789 \\ \mathrm{~N} & 2.90081 & 0.16526 & 3.47959 \\ \mathrm{C} & 3.38243 & -0.44169 & 2.21761 \\ \mathrm{C} & 2.83548 & 0.25250 & 0.95482 \\ \mathrm{C} & 4.85409 & -0.39210 & 2.25538 \\ \mathrm{~N} & 6.01368 & -0.35009 & 2.25783 \\ \mathrm{H} & 3.10367 & 1.31887 & 0.99037 \\ \mathrm{H} & 3.36327 & -0.18824 & 0.10866 \\ \mathrm{H} & 3.57607 & 0.30212 & 4.22217 \\ \mathrm{H} & 1.45433 & 0.84073 & 4.75846 \\ \mathrm{P} & -1.69870 & -0.21278 & 0.70431 \\ \mathrm{P} & 0.62893 & 0.19428 & -1.47378 \\ \mathrm{H} & 0.95987 & -1.37119 & 0.60202 \\ \mathrm{H} & 3.09329 & -1.50104 & 2.21306 \\ \mathrm{C} & -2.24130 & -1.91429 & 1.28445 \\ \mathrm{C} & -2.88198 & 0.88990 & 1.64203 \\ \mathrm{C} & 1.84454 & -0.65085 & -2.61287 \\ \mathrm{C} & 0.70516 & 1.97898 & -2.05525 \\ \mathrm{H} & -3.87931 & 0.72960 & 1.21306 \\ \mathrm{H} & 1.31415 & -0.69967 & -3.57360 \\ \mathrm{H} & 0.32872 & 2.59077 & -1.22490 \\ \mathrm{H} & -1.42126 & -2.59816 & 1.03285 \\ \mathrm{C} & -1.01687 & -0.51182 & -1.99405 \\ \mathrm{H} & -1.20543 & -0.27088 & -3.04489\end{array}$


Supporting Information for Donoghue et al.: Ligand and Substrate Effects on the Mechanisms of the Rhodium...

\begin{tabular}{|c|c|c|c|}
\hline $\mathrm{H}$ & -0.91367 & -1.60142 & -1.93019 \\
\hline $\mathrm{C}$ & -2.16159 & -0.02589 & -1.09203 \\
\hline $\mathrm{H}$ & -3.09191 & -0.55240 & -1.32321 \\
\hline $\mathrm{H}$ & -2.35115 & 1.04095 & -1.25816 \\
\hline $\mathrm{C}$ & 3.09947 & 0.22051 & -2.84306 \\
\hline $\mathrm{H}$ & 3.69332 & -0.23213 & -3.64315 \\
\hline $\mathrm{H}$ & 3.72663 & 0.24589 & -1.93922 \\
\hline $\mathrm{C}$ & 2.19515 & 2.33657 & -2.26977 \\
\hline $\mathrm{H}$ & 2.75710 & 2.26456 & -1.32569 \\
\hline $\mathrm{H}$ & 2.26323 & 3.37104 & -2.62066 \\
\hline $\mathrm{C}$ & -2.32839 & -1.84324 & 2.82775 \\
\hline $\mathrm{H}$ & -2.63285 & -2.82188 & 3.21216 \\
\hline $\mathrm{H}$ & -1.34576 & -1.59706 & 3.25925 \\
\hline $\mathrm{C}$ & -2.92762 & 0.43421 & 3.11729 \\
\hline $\mathrm{H}$ & -1.95135 & 0.61635 & 3.59327 \\
\hline $\mathrm{H}$ & -3.68026 & 1.02653 & 3.64744 \\
\hline $\mathrm{C}$ & 2.20805 & -2.07479 & -2.17145 \\
\hline $\mathrm{H}$ & 2.86695 & -2.53743 & -2.91361 \\
\hline $\mathrm{H}$ & 2.73008 & -2.07883 & -1.21018 \\
\hline $\mathrm{H}$ & 1.32547 & -2.71472 & -2.07828 \\
\hline $\mathrm{C}$ & -0.11916 & 2.27083 & -3.32016 \\
\hline $\mathrm{H}$ & -0.01541 & 3.33023 & -3.57834 \\
\hline $\mathrm{H}$ & 0.24219 & 1.69245 & -4.17425 \\
\hline $\mathrm{H}$ & -1.18557 & 2.07276 & -3.18411 \\
\hline $\mathrm{C}$ & -3.55013 & -2.43076 & 0.67047 \\
\hline $\mathrm{H}$ & -3.78439 & -3.41492 & 1.09118 \\
\hline $\mathrm{H}$ & -4.39147 & -1.77191 & 0.89993 \\
\hline $\mathrm{H}$ & -3.48299 & -2.55248 & -0.41438 \\
\hline $\mathrm{C}$ & -2.50583 & 2.37334 & 1.53161 \\
\hline $\mathrm{H}$ & -3.23036 & 2.98919 & 2.07495 \\
\hline $\mathrm{H}$ & -1.51673 & 2.56551 & 1.96219 \\
\hline $\mathrm{H}$ & -2.50108 & 2.72428 & 0.49471 \\
\hline 0 & 2.79820 & 1.53670 & -3.27073 \\
\hline $\mathrm{O}$ & -3.30982 & -0.92176 & 3.27298 \\
\hline
\end{tabular}

\section{TMBOP Ligand, Substrate a, Minor Manifold}

\begin{tabular}{llll} 
4a-sqpl & & \\
SCF Energy & $=-1832.448776$ & hartree & \\
Total Enthalpy $=-1831.926422$ & hartree & \\
\multicolumn{5}{c}{} & & \\
Rh & 0.71863 & 0.02165 & -0.43463 \\
$\mathrm{O}$ & 0.50275 & 0.12152 & -2.61295 \\
$\mathrm{C}$ & 1.58944 & 0.02011 & -3.21743 \\
$\mathrm{~N}$ & 2.76122 & -0.05531 & -2.58154 \\
$\mathrm{C}$ & 2.82202 & 0.03579 & -1.13216 \\
$\mathrm{C}$ & 2.49184 & 1.26516 & -0.52110 \\
$\mathrm{C}$ & 3.59097 & -1.00964 & -0.52910 \\
$\mathrm{~N}$ & 4.20840 & -1.88985 & -0.08135 \\
$\mathrm{H}$ & 2.27532 & 2.11322 & -1.16479 \\
$\mathrm{H}$ & 2.85810 & 1.50816 & 0.46687 \\
$\mathrm{H}$ & 3.62162 & -0.14125 & -3.11119 \\
$\mathrm{H}$ & 1.62381 & -0.00465 & -4.31224 \\
$\mathrm{P}$ & 0.71386 & -0.25010 & 1.86414
\end{tabular}


Supporting Information for Donoghue et al.: Ligand and Substrate Effects on the Mechanisms of the Rhodium...

\begin{tabular}{|c|c|c|c|}
\hline $\mathrm{P}$ & -1.61247 & 0.35222 & -0.18770 \\
\hline $\mathrm{C}$ & 2.00864 & 0.38274 & 3.07865 \\
\hline C & 0.65599 & -2.04949 & 2.38054 \\
\hline C & -2.18147 & 1.95629 & -0.95881 \\
\hline C & -2.82654 & -0.87308 & -0.92471 \\
\hline $\mathrm{H}$ & 1.91832 & 1.47734 & 3.07574 \\
\hline $\mathrm{H}$ & 1.62594 & -2.43764 & 2.04451 \\
\hline $\mathrm{H}$ & -2.80508 & -1.74631 & -0.26005 \\
\hline $\mathrm{H}$ & -1.83503 & 1.88433 & -1.99699 \\
\hline C & -0.82968 & 0.60357 & 2.50770 \\
\hline $\mathrm{H}$ & -0.56292 & 1.66645 & 2.54069 \\
\hline $\mathrm{H}$ & -1.04649 & 0.30078 & 3.53544 \\
\hline $\mathrm{C}$ & -2.06645 & 0.39886 & 1.62294 \\
\hline $\mathrm{H}$ & -2.55057 & -0.55486 & 1.85482 \\
\hline $\mathrm{H}$ & -2.80789 & 1.18009 & 1.81774 \\
\hline $\mathrm{C}$ & -4.24699 & -0.26481 & -0.87876 \\
\hline $\mathrm{H}$ & -4.94763 & -0.96406 & -1.34683 \\
\hline $\mathrm{H}$ & -4.57643 & -0.10976 & 0.16152 \\
\hline C & -3.72274 & 2.04478 & -0.98129 \\
\hline $\mathrm{H}$ & -4.01534 & 2.93096 & -1.55402 \\
\hline $\mathrm{H}$ & -4.11246 & 2.17161 & 0.04338 \\
\hline $\mathrm{C}$ & 0.60711 & -2.18782 & 3.91923 \\
\hline $\mathrm{H}$ & 0.69048 & -3.24873 & 4.17622 \\
\hline $\mathrm{H}$ & -0.36444 & -1.83262 & 4.30303 \\
\hline $\mathrm{C}$ & 1.63822 & -0.12764 & 4.49700 \\
\hline $\mathrm{H}$ & 2.39224 & 0.23798 & 5.20120 \\
\hline $\mathrm{H}$ & 0.66418 & 0.25965 & 4.83002 \\
\hline $\mathrm{C}$ & -2.44603 & -1.31130 & -2.34687 \\
\hline $\mathrm{H}$ & -3.15329 & -2.07332 & -2.69079 \\
\hline $\mathrm{H}$ & -2.49469 & -0.47478 & -3.04769 \\
\hline $\mathrm{H}$ & -1.44043 & -1.73580 & -2.38836 \\
\hline $\mathrm{C}$ & -1.55096 & 3.18934 & -0.29978 \\
\hline $\mathrm{H}$ & -1.84928 & 4.09627 & -0.83609 \\
\hline $\mathrm{H}$ & -1.87328 & 3.30668 & 0.74107 \\
\hline $\mathrm{H}$ & -0.45857 & 3.13462 & -0.31478 \\
\hline C & -0.45345 & -2.87156 & 1.71173 \\
\hline $\mathrm{H}$ & -0.33297 & -3.93129 & 1.95956 \\
\hline $\mathrm{H}$ & -1.45004 & -2.57176 & 2.05331 \\
\hline $\mathrm{H}$ & -0.41771 & -2.78258 & 0.62174 \\
\hline C & 3.45975 & -0.01950 & 2.76654 \\
\hline $\mathrm{H}$ & 4.11299 & 0.39149 & 3.54362 \\
\hline $\mathrm{H}$ & 3.58940 & -1.10301 & 2.77286 \\
\hline $\mathrm{H}$ & 3.82068 & 0.35515 & 1.80936 \\
\hline O & -4.34390 & 0.94206 & -1.61352 \\
\hline O & 1.67286 & -1.53927 & 4.58552 \\
\hline
\end{tabular}

$4 a-\operatorname{molh}-\mathrm{A}$

SCF Energy = $\quad-1833.622683$ hartree Total Enthalpy $=-1833.084455$ hartree

$\begin{array}{rrrr}\text { Rh } & -0.15211 & 1.03419 & -0.43303 \\ \mathrm{O} & -0.22170 & 0.96089 & -2.62321 \\ \mathrm{C} & 0.89371 & 1.18863 & -3.13548 \\ \mathrm{~N} & 1.96479 & 1.49373 & -2.40265 \\ \mathrm{C} & 1.81587 & 1.78303 & -0.98012 \\ \mathrm{C} & 0.97343 & 2.87918 & -0.61060\end{array}$


Supporting Information for Donoghue et al.: Ligand and Substrate Effects on the Mechanisms of the Rhodium...

\begin{tabular}{|c|c|c|c|}
\hline $\mathrm{C}$ & 2.99911 & 1.45088 & -0.24127 \\
\hline $\mathrm{N}$ & 3.98938 & 1.20682 & 0.31907 \\
\hline $\mathrm{H}$ & 0.57465 & 3.50855 & -1.40118 \\
\hline $\mathrm{H}$ & 1.16340 & 3.38300 & 0.32974 \\
\hline $\mathrm{H}$ & 2.85282 & 1.68491 & -2.85475 \\
\hline $\mathrm{H}$ & 1.03418 & 1.13768 & -4.22035 \\
\hline $\mathrm{P}$ & -0.15587 & 0.84699 & 1.88988 \\
\hline $\mathrm{P}$ & -0.75848 & -1.30325 & -0.38044 \\
\hline $\mathrm{H}$ & -2.07666 & 1.33188 & -0.38108 \\
\hline $\mathrm{H}$ & -1.75782 & 2.05641 & -0.38765 \\
\hline $\mathrm{C}$ & -1.27007 & 2.09449 & 2.74745 \\
\hline $\mathrm{C}$ & 1.42702 & 0.96069 & 2.88112 \\
\hline $\mathrm{C}$ & -1.86110 & -1.93713 & -1.75060 \\
\hline $\mathrm{C}$ & 0.55909 & -2.63801 & -0.32436 \\
\hline $\mathrm{H}$ & 1.02770 & -2.56616 & 0.66578 \\
\hline $\mathrm{H}$ & -1.28703 & -1.72475 & -2.66115 \\
\hline $\mathrm{H}$ & -2.29627 & 1.79040 & 2.49958 \\
\hline $\mathrm{H}$ & 1.92713 & 1.86192 & 2.50821 \\
\hline $\mathrm{C}$ & -1.68884 & -1.48188 & 1.22424 \\
\hline $\mathrm{H}$ & -1.90349 & -2.52646 & 1.47083 \\
\hline $\mathrm{H}$ & -2.65245 & -0.97892 & 1.08990 \\
\hline $\mathrm{C}$ & -0.88832 & -0.82245 & 2.35899 \\
\hline $\mathrm{H}$ & -1.51042 & -0.70481 & 3.25145 \\
\hline $\mathrm{H}$ & -0.05209 & -1.46607 & 2.64584 \\
\hline $\mathrm{C}$ & -1.08132 & 1.98917 & 4.27879 \\
\hline $\mathrm{H}$ & -1.69654 & 2.75467 & 4.76239 \\
\hline $\mathrm{H}$ & -1.41161 & 1.01046 & 4.66082 \\
\hline $\mathrm{C}$ & 1.12811 & 1.16802 & 4.38637 \\
\hline $\mathrm{H}$ & 2.06994 & 1.39211 & 4.89680 \\
\hline $\mathrm{H}$ & 0.73111 & 0.23377 & 4.81738 \\
\hline $\mathrm{C}$ & -2.04764 & -3.46665 & -1.66572 \\
\hline $\mathrm{H}$ & -2.58997 & -3.80591 & -2.55444 \\
\hline $\mathrm{H}$ & -2.65821 & -3.72865 & -0.78502 \\
\hline $\mathrm{C}$ & -0.09106 & -4.03306 & -0.45239 \\
\hline $\mathrm{H}$ & 0.69625 & -4.79378 & -0.47944 \\
\hline $\mathrm{H}$ & -0.73098 & -4.25093 & 0.41801 \\
\hline $\mathrm{C}$ & -1.04105 & 3.54233 & 2.28632 \\
\hline $\mathrm{H}$ & -1.76219 & 4.19821 & 2.78480 \\
\hline $\mathrm{H}$ & -0.04213 & 3.88891 & 2.56500 \\
\hline $\mathrm{H}$ & -1.16923 & 3.66273 & 1.20892 \\
\hline $\mathrm{C}$ & 2.35858 & -0.24826 & 2.69334 \\
\hline $\mathrm{H}$ & 3.35946 & -0.00276 & 3.05903 \\
\hline $\mathrm{H}$ & 2.00543 & -1.11570 & 3.26135 \\
\hline $\mathrm{H}$ & 2.46682 & -0.53621 & 1.64671 \\
\hline $\mathrm{C}$ & 1.63301 & -2.41685 & -1.40118 \\
\hline $\mathrm{H}$ & 2.42202 & -3.16907 & -1.29772 \\
\hline $\mathrm{H}$ & 1.21219 & -2.52280 & -2.40522 \\
\hline $\mathrm{H}$ & 2.09544 & -1.43022 & -1.31214 \\
\hline $\mathrm{C}$ & -3.20666 & -1.20586 & -1.84443 \\
\hline $\mathrm{H}$ & -3.77117 & -1.57808 & -2.70606 \\
\hline $\mathrm{H}$ & -3.82867 & -1.36605 & -0.95713 \\
\hline $\mathrm{H}$ & -3.07077 & -0.13047 & -1.98570 \\
\hline O & 0.25534 & 2.24435 & 4.66518 \\
\hline O & -0.82593 & -4.18270 & -1.65343 \\
\hline
\end{tabular}

4a-molh-C 
Supporting Information for Donoghue et al.: Ligand and Substrate Effects on the Mechanisms of the Rhodium...

$\begin{array}{lll}\text { SCF Energy } & =-1833.623571 \text { hartree } \\ \text { Total Enthalpy } & =-1833.085532 \text { hartree }\end{array}$

\begin{tabular}{|c|c|c|c|}
\hline $\mathrm{Rh}$ & 0.70672 & -0.20976 & -0.44721 \\
\hline O & 0.45862 & 0.01753 & -2.61932 \\
\hline $\mathrm{C}$ & 1.54099 & 0.15615 & -3.22898 \\
\hline $\mathrm{N}$ & 2.70863 & 0.22550 & -2.59495 \\
\hline $\mathrm{C}$ & 2.75689 & 0.24611 & -1.13918 \\
\hline $\mathrm{C}$ & 2.15210 & 1. 36252 & -0.47789 \\
\hline $\mathrm{C}$ & 3.84108 & -0.53854 & -0.63524 \\
\hline $\mathrm{N}$ & 4.73707 & -1.20244 & -0.29996 \\
\hline $\mathrm{H}$ & 1.81388 & 2.18723 & -1.09989 \\
\hline $\mathrm{H}$ & 2.50840 & 1.65157 & 0.50366 \\
\hline $\mathrm{H}$ & 3.57002 & 0.32056 & -3.12172 \\
\hline $\mathrm{H}$ & 1.56335 & 0.22601 & -4.32176 \\
\hline P & 0.67351 & -0.37037 & 1.88405 \\
\hline $\mathrm{P}$ & -1.60003 & 0.29710 & -0.22013 \\
\hline $\mathrm{H}$ & 0.18766 & -2.23298 & -0.90267 \\
\hline $\mathrm{H}$ & 0.94979 & -2.22001 & -0.81866 \\
\hline $\mathrm{C}$ & 1.99056 & 0.35139 & 3.02113 \\
\hline $\mathrm{C}$ & 0.55915 & -2.11696 & 2.56751 \\
\hline $\mathrm{C}$ & -2.06284 & 1.95157 & -0.95972 \\
\hline $\mathrm{C}$ & -2.88581 & -0.82892 & -0.99112 \\
\hline $\mathrm{H}$ & 1.89738 & 1.44235 & 2.93242 \\
\hline $\mathrm{H}$ & 1.47854 & -2.59773 & 2.21028 \\
\hline $\mathrm{H}$ & -2.92192 & -1.71794 & -0.34699 \\
\hline $\mathrm{H}$ & -1.69759 & 1.88967 & -1.99211 \\
\hline $\mathrm{C}$ & -0.84885 & 0.55343 & 2.47394 \\
\hline $\mathrm{H}$ & -0.55462 & 1.60930 & 2.47647 \\
\hline $\mathrm{H}$ & -1.08016 & 0.28754 & 3.50929 \\
\hline C & -2.07859 & 0.35106 & 1.58199 \\
\hline $\mathrm{H}$ & -2.56821 & -0.60097 & 1.80958 \\
\hline $\mathrm{H}$ & -2.81791 & 1.13650 & 1.76812 \\
\hline $\mathrm{C}$ & -4.26689 & -0.13654 & -0.94247 \\
\hline $\mathrm{H}$ & -5.00715 & -0.78308 & -1.42518 \\
\hline $\mathrm{H}$ & -4.59327 & 0.02579 & 0.09751 \\
\hline $\mathrm{C}$ & -3.59633 & 2.13479 & -1.00801 \\
\hline $\mathrm{H}$ & -3.82249 & 3.04529 & -1.57291 \\
\hline $\mathrm{H}$ & -3.99221 & 2.27438 & 0.01269 \\
\hline $\mathrm{C}$ & 0.59798 & -2.12061 & 4.11640 \\
\hline $\mathrm{H}$ & 0.67697 & -3.15951 & 4.45286 \\
\hline $\mathrm{H}$ & -0.34923 & -1.71823 & 4.51448 \\
\hline C & 1.65713 & -0.03936 & 4.48324 \\
\hline $\mathrm{H}$ & 2.42772 & 0.38344 & 5.13562 \\
\hline $\mathrm{H}$ & 0.68904 & 0.36971 & 4.80905 \\
\hline $\mathrm{C}$ & -2.53483 & -1.25463 & -2.42541 \\
\hline $\mathrm{H}$ & -3.27532 & -1.98057 & -2.77739 \\
\hline $\mathrm{H}$ & -2.55827 & -0.40100 & -3.10656 \\
\hline $\mathrm{H}$ & -1.54780 & -1.71643 & -2.49284 \\
\hline C & -1.38875 & 3.13437 & -0.25236 \\
\hline $\mathrm{H}$ & -1.61180 & 4.06663 & -0.78204 \\
\hline $\mathrm{H}$ & -1.74907 & 3.25271 & 0.77589 \\
\hline $\mathrm{H}$ & -0.30235 & 3.01992 & -0.22042 \\
\hline C & -0.65113 & -2.92915 & 2.08565 \\
\hline $\mathrm{H}$ & -0.56022 & -3.96425 & 2.43106 \\
\hline $\mathrm{H}$ & -1.58912 & -2.53686 & 2.4921 \\
\hline
\end{tabular}


Supporting Information for Donoghue et al.: Ligand and Substrate Effects on the Mechanisms of the Rhodium...

$\begin{array}{rrrr}\mathrm{H} & -0.73125 & -2.95611 & 0.99732 \\ \mathrm{C} & 3.43022 & -0.07302 & 2.69733 \\ \mathrm{H} & 4.10198 & 0.34757 & 3.45314 \\ \mathrm{H} & 3.55175 & -1.15786 & 2.72684 \\ \mathrm{H} & 3.77220 & 0.27869 & 1.72642 \\ \mathrm{O} & -4.27931 & 1.08358 & -1.66191 \\ \mathrm{O} & 1.69882 & -1.43969 & 4.67818\end{array}$

$4 a-m o l h-t s-A$

SCF Energy $=-1833.614653$ hartree Total Enthalpy $=-1833.077851$ hartree Negative Frequencies: $-805.31 \mathrm{~cm}^{-1}$

\begin{tabular}{|c|c|c|c|}
\hline $\mathrm{Rh}$ & -0.11122 & -0.85511 & 0.86255 \\
\hline O & 1.54329 & -2.07397 & 1.64655 \\
\hline $\mathrm{C}$ & 1.79284 & -3.09233 & 0.97234 \\
\hline $\mathrm{N}$ & 1.09807 & -3.43024 & -0.11889 \\
\hline $\mathrm{C}$ & -0.10573 & -2.71043 & -0.48648 \\
\hline $\mathrm{C}$ & -1.19030 & -2.72568 & 0.42298 \\
\hline $\mathrm{C}$ & -0.27516 & -2.54190 & -1.89527 \\
\hline $\mathrm{N}$ & -0.39918 & -2.43976 & -3.04880 \\
\hline $\mathrm{H}$ & -1.13406 & -3.37991 & 1.28629 \\
\hline $\mathrm{H}$ & -2.18771 & -2.51651 & 0.05604 \\
\hline $\mathrm{H}$ & 1. 34755 & -4.26939 & -0.63119 \\
\hline $\mathrm{H}$ & 2.61763 & -3.75737 & 1.25044 \\
\hline $\mathrm{P}$ & -1.71560 & 0.65484 & 0.18034 \\
\hline $\mathrm{P}$ & 1.51489 & 0.78445 & -0.01424 \\
\hline $\mathrm{H}$ & -0.15557 & 0.02546 & 2.19969 \\
\hline $\mathrm{H}$ & -0.91067 & -0.96854 & 2.24348 \\
\hline $\mathrm{C}$ & -3.20515 & 0.82265 & 1.30000 \\
\hline $\mathrm{C}$ & -2.49998 & 0.50067 & -1.51021 \\
\hline $\mathrm{C}$ & 2.85637 & 1.23664 & 1.21621 \\
\hline $\mathrm{C}$ & 2.53274 & 0.59624 & -1.58291 \\
\hline $\mathrm{C}$ & -0.88141 & 2.32234 & 0.27051 \\
\hline $\mathrm{H}$ & -0.83754 & 2.55580 & 1.34000 \\
\hline $\mathrm{H}$ & -1.51342 & 3.08473 & -0.19724 \\
\hline $\mathrm{C}$ & 0.53212 & 2.35161 & -0.33735 \\
\hline $\mathrm{H}$ & 0.47475 & 2.46765 & -1.42316 \\
\hline $\mathrm{H}$ & 1.07389 & 3.22451 & 0.03971 \\
\hline $\mathrm{C}$ & 3.53915 & 1.76206 & -1.69217 \\
\hline $\mathrm{H}$ & 3.01796 & 2.72892 & -1.78260 \\
\hline $\mathrm{H}$ & 4.14525 & 1.62886 & -2.59449 \\
\hline $\mathrm{C}$ & 3.86915 & 2.22981 & 0.60687 \\
\hline $\mathrm{H}$ & 4.69834 & 2.35948 & 1.31003 \\
\hline $\mathrm{H}$ & 3.39777 & 3.21850 & 0.47077 \\
\hline $\mathrm{C}$ & -3.72813 & 1.44023 & -1.63115 \\
\hline $\mathrm{H}$ & -4.24432 & 1.20365 & -2.56685 \\
\hline $\mathrm{H}$ & -3.38854 & 2.48770 & -1.69710 \\
\hline $\mathrm{C}$ & -4.23038 & 1.78090 & 0.65077 \\
\hline $\mathrm{H}$ & -5.11248 & 1.84368 & 1.29553 \\
\hline $\mathrm{H}$ & -3.82066 & 2.79791 & 0.54751 \\
\hline 0 & 4.44788 & 1.78864 & -0.60642 \\
\hline O & -4.68202 & 1.29680 & -0.60071 \\
\hline $\mathrm{C}$ & 2.31304 & 1.75658 & 2.55217 \\
\hline $\mathrm{H}$ & 1.67494 & 1.01730 & 3.04192 \\
\hline $\mathrm{H}$ & 1.74036 & 2.68320 & 2.43119 \\
\hline
\end{tabular}


Supporting Information for Donoghue et al.: Ligand and Substrate Effects on the Mechanisms of the Rhodium...

$\begin{array}{rrrr}\mathrm{H} & 3.14390 & 1.97640 & 3.23086 \\ \mathrm{C} & 3.24801 & -0.76060 & -1.65590 \\ \mathrm{H} & 2.54155 & -1.59211 & -1.60808 \\ \mathrm{H} & 3.98378 & -0.86605 & -0.85394 \\ \mathrm{H} & 3.78847 & -0.83856 & -2.60519 \\ \mathrm{C} & -1.53911 & 0.74964 & -2.68183 \\ \mathrm{H} & -0.64745 & 0.12330 & -2.63074 \\ \mathrm{H} & -1.23956 & 1.80158 & -2.73407 \\ \mathrm{H} & -2.04151 & 0.50722 & -3.62359 \\ \mathrm{C} & -3.86717 & -0.52321 & 1.63576 \\ \mathrm{H} & -3.16855 & -1.21733 & 2.10707 \\ \mathrm{H} & -4.28982 & -0.99029 & 0.74184 \\ \mathrm{H} & -4.69366 & -0.35341 & 2.33333 \\ \mathrm{H} & 3.38108 & 0.29006 & 1.39407 \\ \mathrm{H} & 1.82109 & 0.67142 & -2.41495 \\ \mathrm{H} & -2.86358 & -0.53304 & -1.55678 \\ \mathrm{H} & -2.82636 & 1.28140 & 2.22272\end{array}$

$4 a-m o l h-t s-C$

SCF Energy $=-1833.610121$ hartree
Total Enthalpy $=-1833.073282$ hartree
Negative Frequencies: $-847.78 \mathrm{~cm}^{-1}$

$\begin{array}{rrrr}\text { Rh } & 0.04239 & -0.98115 & 0.38710 \\ \mathrm{O} & -1.27073 & -2.68844 & 0.84538 \\ \mathrm{C} & -0.78442 & -3.77985 & 0.48796 \\ \mathrm{~N} & 0.33645 & -3.86215 & -0.23157 \\ \mathrm{C} & 0.94847 & -2.66095 & -0.77182 \\ \mathrm{C} & 0.18519 & -1.87067 & -1.66375 \\ \mathrm{C} & 2.38197 & -2.71081 & -0.76422 \\ \mathrm{~N} & 3.54220 & -2.79895 & -0.75524 \\ \mathrm{H} & -0.78516 & -2.24500 & -1.97102 \\ \mathrm{H} & 0.68523 & -1.21076 & -2.35849 \\ \mathrm{H} & 0.71644 & -4.76745 & -0.48495 \\ \mathrm{H} & -1.26775 & -4.72419 & 0.76014 \\ \mathrm{P} & 1.43802 & 0.87827 & 0.22994 \\ \mathrm{P} & -1.79162 & 0.60281 & -0.03728 \\ \mathrm{H} & -0.05295 & -0.54888 & 1.92853 \\ \mathrm{H} & 0.87511 & -1.39716 & 1.69456 \\ \mathrm{C} & 2.23856 & 1.42310 & -1.38289 \\ \mathrm{C} & 2.89374 & 0.95609 & 1.39789 \\ \mathrm{C} & -2.95589 & 0.47375 & -1.50035 \\ \mathrm{C} & -3.00389 & 0.78471 & 1.38407 \\ \mathrm{C} & 0.31821 & 2.30493 & 0.67521 \\ \mathrm{H} & 0.83625 & 3.26019 & 0.55329 \\ \mathrm{H} & 0.08925 & 2.19781 & 1.74054 \\ \mathrm{C} & -0.97371 & 2.27901 & -0.15686 \\ \mathrm{H} & -1.65227 & 3.07019 & 0.17583 \\ \mathrm{H} & -0.75444 & 2.47536 & -1.21149 \\ \mathrm{C} & -4.14763 & 1.74354 & 0.98824 \\ \mathrm{H} & -4.87222 & 1.78845 & 1.80773 \\ \mathrm{H} & -3.77473 & 2.76689 & 0.82104 \\ \mathrm{C} & -4.15109 & 1.44275 & -1.35909 \\ \mathrm{H} & -4.86604 & 1.23817 & -2.16298 \\ \mathrm{H} & -3.80688 & 2.48425 & -1.47913 \\ \mathrm{O} & -4.86357 & 1.29210 & -0.14793\end{array}$


Supporting Information for Donoghue et al.: Ligand and Substrate Effects on the Mechanisms of the Rhodium...

$\begin{array}{rrrr}\mathrm{C} & 3.10283 & 2.67987 & -1.09639 \\ \mathrm{H} & 3.57522 & 2.99042 & -2.03377 \\ \mathrm{H} & 2.49457 & 3.52469 & -0.73965 \\ \mathrm{C} & 3.72674 & 2.23976 & 1.14353 \\ \mathrm{H} & 4.63720 & 2.17806 & 1.74711 \\ \mathrm{H} & 3.16451 & 3.12585 & 1.48222 \\ \mathrm{O} & 4.15342 & 2.40424 & -0.19376 \\ \mathrm{C} & 3.11188 & 0.36210 & -2.07191 \\ \mathrm{H} & 2.55905 & -0.52920 & -2.36321 \\ \mathrm{H} & 3.94479 & 0.05035 & -1.43963 \\ \mathrm{H} & 3.53732 & 0.79538 & -2.98323 \\ \mathrm{C} & 2.53501 & 0.84989 & 2.88562 \\ \mathrm{H} & 2.02370 & -0.08550 & 3.12024 \\ \mathrm{H} & 1.90510 & 1.68121 & 3.21947 \\ \mathrm{H} & 3.45281 & 0.87859 & 3.48187 \\ \mathrm{C} & -2.26609 & 0.64933 & -2.85906 \\ \mathrm{H} & -1.40500 & -0.01332 & -2.97176 \\ \mathrm{H} & -1.92590 & 1.67878 & -3.01424 \\ \mathrm{H} & -2.96858 & 0.41859 & -3.66654 \\ \mathrm{C} & -3.55766 & -0.56871 & 1.85816 \\ \mathrm{H} & -2.76447 & -1.26922 & 2.12167 \\ \mathrm{H} & -4.18339 & -1.02742 & 1.08765 \\ \mathrm{H} & -4.18746 & -0.41250 & 2.74031 \\ \mathrm{H} & 1.41677 & 1.71344 & -2.05027 \\ \mathrm{H} & -2.42959 & 1.24536 & 2.19932 \\ \mathrm{H} & -3.35148 & -0.54752 & -1.42808 \\ \mathrm{H} & 3.51051 & 0.09437 & 1.11359\end{array}$

$4 a-d i h y-A$

SCF Energy $\quad=-1833.620147$ hartree Total Enthalpy $=-1833.080556$ hartree

$\begin{array}{rrrr}\text { Rh } & -0.09134 & 1.01672 & -0.31229 \\ \mathrm{O} & -0.53906 & 0.96274 & -2.47891 \\ \mathrm{C} & 0.43308 & 0.95171 & -3.24726 \\ \mathrm{~N} & 1.71214 & 0.98289 & -2.82769 \\ \mathrm{C} & 2.05562 & 1.12801 & -1.43480 \\ \mathrm{C} & 1.78363 & 2.32314 & -0.78591 \\ \mathrm{C} & 2.97146 & 0.15174 & -0.92818 \\ \mathrm{~N} & 3.74052 & -0.63820 & -0.55308 \\ \mathrm{H} & 1.42771 & 3.16865 & -1.36089 \\ \mathrm{H} & 2.23634 & 2.53390 & 0.17444 \\ \mathrm{H} & 2.45887 & 0.93411 & -3.51217 \\ \mathrm{H} & 0.29282 & 0.90634 & -4.33349 \\ \mathrm{P} & -0.08510 & 1.13139 & 1.98647 \\ \mathrm{P} & -0.30659 & -1.46163 & -0.07046 \\ \mathrm{H} & -1.60155 & 1.01362 & 0.02123 \\ \mathrm{H} & -0.49601 & 2.53317 & -0.26507 \\ \mathrm{C} & -1.24388 & 2.38661 & 2.75480 \\ \mathrm{C} & 1.49975 & 1.46842 & 2.92729 \\ \mathrm{C} & -1.94660 & -2.17218 & -0.64933 \\ \mathrm{C} & 0.86336 & -2.78068 & -0.72339 \\ \mathrm{H} & 1.81489 & -2.63653 & -0.19735 \\ \mathrm{H} & -1.93904 & -1.99936 & -1.73287 \\ \mathrm{H} & 1.87851 & 2.40845 & 2.50651 \\ \mathrm{C} & -0.73206 & -0.51147 & 2.59395\end{array}$


Supporting Information for Donoghue et al.: Ligand and Substrate Effects on the Mechanisms of the Rhodium...

\begin{tabular}{|c|c|c|c|}
\hline $\mathrm{H}$ & -1.82317 & -0.43369 & 2.53632 \\
\hline $\mathrm{H}$ & -0.47999 & -0.64054 & 3.65150 \\
\hline $\mathrm{C}$ & -0.23969 & -1.72154 & 1.78362 \\
\hline $\mathrm{H}$ & 0.80854 & -1.93465 & 2.01840 \\
\hline $\mathrm{H}$ & -0.81178 & -2.60819 & 2.07317 \\
\hline $\mathrm{C}$ & -1.06522 & 2.37289 & 4.29032 \\
\hline $\mathrm{H}$ & -1.73107 & 3.12160 & 4.73027 \\
\hline $\mathrm{H}$ & -1.33585 & 1.39522 & 4.71906 \\
\hline $\mathrm{C}$ & 1.20305 & 1.72587 & 4.42721 \\
\hline $\mathrm{H}$ & 2.13192 & 2.05552 & 4.90371 \\
\hline $\mathrm{H}$ & 0.90150 & 0.78331 & 4.91464 \\
\hline $\mathrm{C}$ & -2.01222 & -3.69881 & -0.41881 \\
\hline $\mathrm{H}$ & -2.92571 & -4.08185 & -0.88567 \\
\hline $\mathrm{H}$ & -2.08242 & -3.91576 & 0.66109 \\
\hline $\mathrm{C}$ & 0.31026 & -4.18138 & -0.38441 \\
\hline $\mathrm{H}$ & 0.99284 & -4.93966 & -0.78256 \\
\hline $\mathrm{H}$ & 0.24476 & -4.33469 & 0.70437 \\
\hline $\mathrm{H}$ & -2.25566 & 2.03486 & 2.51545 \\
\hline $\mathrm{C}$ & -1.05977 & 3.80950 & 2.20619 \\
\hline $\mathrm{H}$ & -1.78199 & 4.47589 & 2.68894 \\
\hline $\mathrm{H}$ & -0.06221 & 4.19835 & 2.42753 \\
\hline $\mathrm{H}$ & -1.22961 & 3.85629 & 1.12883 \\
\hline $\mathrm{C}$ & 2.56609 & 0.37339 & 2.78996 \\
\hline $\mathrm{H}$ & 3.48993 & 0.69498 & 3.28191 \\
\hline $\mathrm{H}$ & 2.24992 & -0.55411 & 3.27877 \\
\hline $\mathrm{H}$ & 2.81267 & 0.14293 & 1.75272 \\
\hline $\mathrm{C}$ & 1.10155 & -2.65334 & -2.23652 \\
\hline $\mathrm{H}$ & 1.83281 & -3.40460 & -2.55324 \\
\hline $\mathrm{H}$ & 0.18362 & -2.83439 & -2.80157 \\
\hline $\mathrm{H}$ & 1.50013 & -1.67420 & -2.50718 \\
\hline $\mathrm{C}$ & -3.18174 & -1.48576 & -0.05356 \\
\hline $\mathrm{H}$ & -4.09068 & -1.91396 & -0.48952 \\
\hline $\mathrm{H}$ & -3.24668 & -1.62869 & 1.03095 \\
\hline $\mathrm{H}$ & -3.19088 & -0.41331 & -0.26363 \\
\hline O & 0.24955 & 2.73597 & 4.67237 \\
\hline 0 & -0.94407 & -4.42200 & -0.99832 \\
\hline
\end{tabular}

$4 a-d i h y-c$

SCF Energy $\quad=-1833.616410$ hartree

Total Enthalpy $=-1833.076921$ hartree

$\begin{array}{rrrr}\mathrm{Rh} & 0.52961 & -0.61540 & -0.46952 \\ \mathrm{O} & 0.30702 & -0.78400 & -2.66984 \\ \mathrm{C} & 1.36199 & -0.84937 & -3.31573 \\ \mathrm{~N} & 2.57020 & -0.62332 & -2.77286 \\ \mathrm{C} & 2.72825 & -0.19665 & -1.40570 \\ \mathrm{C} & 2.23385 & 1.02186 & -0.99452 \\ \mathrm{C} & 3.74234 & -0.92618 & -0.69565 \\ \mathrm{~N} & 4.61611 & -1.50765 & -0.19582 \\ \mathrm{H} & 1.76128 & 1.67859 & -1.71360 \\ \mathrm{H} & 2.53444 & 1.43697 & -0.04234 \\ \mathrm{H} & 3.40578 & -0.77953 & -3.32566 \\ \mathrm{H} & 1.35976 & -1.10081 & -4.38263 \\ \mathrm{P} & 0.46603 & -0.86723 & 1.82013 \\ \mathrm{P} & -1.18010 & 1.17938 & -0.13916 \\ \mathrm{H} & -0.71737 & -1.51336 & -0.29861\end{array}$


Supporting Information for Donoghue et al.: Ligand and Substrate Effects on the Mechanisms of the Rhodium...

\begin{tabular}{|c|c|c|c|}
\hline $\mathrm{H}$ & 1.15858 & -2.06272 & -0.49240 \\
\hline $\mathrm{C}$ & 1.60160 & 0.08416 & 2.97496 \\
\hline $\mathrm{C}$ & 0.63896 & -2.60324 & 2.47962 \\
\hline $\mathrm{C}$ & -1.01599 & 3.00681 & -0.54673 \\
\hline $\mathrm{C}$ & -2.83161 & 0.78265 & -0.94260 \\
\hline $\mathrm{H}$ & 1.30822 & 1.13935 & 2.89628 \\
\hline $\mathrm{H}$ & 1.64149 & -2.90722 & 2.15427 \\
\hline $\mathrm{H}$ & -3.18722 & -0.13047 & -0.44668 \\
\hline $\mathrm{H}$ & -0.74057 & 3.01586 & -1.60944 \\
\hline $\mathrm{C}$ & -1.24034 & -0.27291 & 2.29028 \\
\hline $\mathrm{H}$ & -1.36042 & -0.25498 & 3.37738 \\
\hline $\mathrm{H}$ & -1.94638 & -1.01322 & 1.90078 \\
\hline $\mathrm{C}$ & -1.53322 & 1.11627 & 1.69865 \\
\hline $\mathrm{H}$ & -2.57046 & 1.39755 & 1.90357 \\
\hline $\mathrm{H}$ & -0.90463 & 1.87154 & 2.18186 \\
\hline $\mathrm{C}$ & -3.84679 & 1.91344 & -0.67644 \\
\hline $\mathrm{H}$ & -4.77905 & 1.68424 & -1.20268 \\
\hline $\mathrm{H}$ & -4.08554 & 1.99818 & 0.39578 \\
\hline $\mathrm{C}$ & -2.36836 & 3.74319 & -0.41841 \\
\hline $\mathrm{H}$ & -2.24862 & 4.76590 & -0.79167 \\
\hline $\mathrm{H}$ & -2.65831 & 3.81098 & 0.64408 \\
\hline $\mathrm{C}$ & 0.62703 & -2.60788 & 4.02649 \\
\hline $\mathrm{H}$ & 0.86119 & -3.61978 & 4.37062 \\
\hline $\mathrm{H}$ & -0.37903 & -2.35580 & 4.40108 \\
\hline $\mathrm{C}$ & 1.33463 & -0.38404 & 4.42935 \\
\hline $\mathrm{H}$ & 2.02069 & 0.15084 & 5.09416 \\
\hline $\mathrm{H}$ & 0.30998 & -0.14150 & 4.75073 \\
\hline $\mathrm{C}$ & -2.69133 & 0.51694 & -2.45049 \\
\hline $\mathrm{H}$ & -3.66175 & 0.21256 & -2.85643 \\
\hline $\mathrm{H}$ & -2.38963 & 1. 42482 & -2.98062 \\
\hline $\mathrm{H}$ & -1.96833 & -0.27036 & -2.66450 \\
\hline $\mathrm{C}$ & 0.06898 & 3.73308 & 0.25982 \\
\hline $\mathrm{H}$ & 0.22161 & 4.74053 & -0.14071 \\
\hline $\mathrm{H}$ & -0.21349 & 3.84644 & 1.31167 \\
\hline $\mathrm{H}$ & 1.03177 & 3.21800 & 0.22506 \\
\hline $\mathrm{C}$ & -0.38934 & -3.59095 & 1.91591 \\
\hline $\mathrm{H}$ & -0.17108 & -4.59779 & 2.28616 \\
\hline $\mathrm{H}$ & -1.41018 & -3.34396 & 2.22638 \\
\hline $\mathrm{H}$ & -0.35822 & -3.62386 & 0.82446 \\
\hline $\mathrm{C}$ & 3.09391 & -0.05759 & 2.64405 \\
\hline $\mathrm{H}$ & 3.67929 & 0.52503 & 3.36295 \\
\hline $\mathrm{H}$ & 3.42757 & -1.09471 & 2.71675 \\
\hline $\mathrm{H}$ & 3.34130 & 0.30651 & 1.64724 \\
\hline 0 & -3.40748 & 3.16355 & -1.17992 \\
\hline O & 1.59987 & -1.75984 & 4.60818 \\
\hline
\end{tabular}

$4 a-d i h y-t s-A$

SCF Energy = $\quad-1833.615359$ hartree Total Enthalpy $=-1833.077485$ hartree Negative Frequencies: $-609.73 \mathrm{~cm}^{-1}$

$\begin{array}{rrrr}\text { Rh } & -0.08439 & -0.79884 & 0.82534 \\ \mathrm{O} & 1.60889 & -1.90907 & 1.73501 \\ \mathrm{C} & 1.94536 & -2.95387 & 1.14776 \\ \mathrm{~N} & 1.29431 & -3.43800 & 0.08010 \\ \mathrm{C} & 0.07061 & -2.83043 & -0.38251\end{array}$


Supporting Information for Donoghue et al.: Ligand and Substrate Effects on the Mechanisms of the Rhodium...

\begin{tabular}{|c|c|c|c|}
\hline C & -1.05244 & -2.86782 & 0.48153 \\
\hline $\mathrm{C}$ & -0.04693 & -2.68199 & -1.79434 \\
\hline $\mathrm{N}$ & -0.12604 & -2.59318 & -2.95429 \\
\hline $\mathrm{H}$ & -1.00463 & -3.53982 & 1.33312 \\
\hline $\mathrm{H}$ & -2.03943 & -2.72954 & 0.05553 \\
\hline $\mathrm{H}$ & 1.63449 & -4.28008 & -0.37141 \\
\hline $\mathrm{H}$ & 2.81296 & -3.53196 & 1.48484 \\
\hline $\mathrm{P}$ & -1.74856 & 0.64099 & 0.17219 \\
\hline $\mathrm{P}$ & 1.46297 & 0.82516 & -0.06279 \\
\hline $\mathrm{H}$ & -0.17921 & 0.18377 & 2.03328 \\
\hline $\mathrm{H}$ & -1.16254 & -1.62663 & 1.68746 \\
\hline C & -3.21430 & 0.82425 & 1.31940 \\
\hline C & -2.58421 & 0.38514 & -1.48271 \\
\hline C & 2.77591 & 1.34673 & 1.16479 \\
\hline C & 2.49841 & 0.59111 & -1.61056 \\
\hline $\mathrm{C}$ & -0.96106 & 2.33151 & 0.15377 \\
\hline $\mathrm{H}$ & -0.91875 & 2.63350 & 1.20578 \\
\hline $\mathrm{H}$ & -1.61007 & 3.04775 & -0.36034 \\
\hline C & 0.44810 & 2.35126 & -0.46280 \\
\hline $\mathrm{H}$ & 0.38260 & 2.40159 & -1.55350 \\
\hline $\mathrm{H}$ & 0.97803 & 3.25306 & -0.14135 \\
\hline C & 3.46986 & 1.78429 & -1.75589 \\
\hline $\mathrm{H}$ & 2.92087 & 2.73059 & -1.88769 \\
\hline $\mathrm{H}$ & 4.08696 & 1.63463 & -2.64799 \\
\hline $\mathrm{C}$ & 3.76791 & 2.34511 & 0.52838 \\
\hline $\mathrm{H}$ & 4.58749 & 2.51898 & 1.23308 \\
\hline $\mathrm{H}$ & 3.27272 & 3.31623 & 0.35609 \\
\hline $\mathrm{C}$ & -3.82032 & 1.30981 & -1.62177 \\
\hline $\mathrm{H}$ & -4.35881 & 1.02464 & -2.53113 \\
\hline $\mathrm{H}$ & -3.49185 & 2.35538 & -1.74779 \\
\hline C & -4.26709 & 1.74448 & 0.65807 \\
\hline $\mathrm{H}$ & -5.13311 & 1.82445 & 1.32233 \\
\hline $\mathrm{H}$ & -3.87140 & 2.76076 & 0.50488 \\
\hline $\mathrm{O}$ & 4.36789 & 1.87884 & -0.66534 \\
\hline O & -4.74716 & 1.20855 & -0.56135 \\
\hline C & 2.20599 & 1.89404 & 2.47927 \\
\hline $\mathrm{H}$ & 1.58637 & 1.15122 & 2.98654 \\
\hline $\mathrm{H}$ & 1.60612 & 2.79798 & 2.32315 \\
\hline $\mathrm{H}$ & 3.02501 & 2.16134 & 3.15532 \\
\hline C & 3.25892 & -0.74256 & -1.61908 \\
\hline $\mathrm{H}$ & 2.58024 & -1.59457 & -1.54731 \\
\hline $\mathrm{H}$ & 3.98833 & -0.79040 & -0.80596 \\
\hline $\mathrm{H}$ & 3.81253 & -0.83964 & -2.55900 \\
\hline C & -1.65705 & 0.55503 & -2.69438 \\
\hline $\mathrm{H}$ & -0.77749 & -0.08928 & -2.63963 \\
\hline $\mathrm{H}$ & -1.33541 & 1.59562 & -2.80910 \\
\hline $\mathrm{H}$ & -2.19442 & 0.28078 & -3.60805 \\
\hline C & -3.84695 & -0.51773 & 1.71904 \\
\hline $\mathrm{H}$ & -3.13734 & -1.16130 & 2.24362 \\
\hline $\mathrm{H}$ & -4.24415 & -1.04888 & 0.84939 \\
\hline $\mathrm{H}$ & -4.68818 & -0.33267 & 2.39480 \\
\hline $\mathrm{H}$ & 3.32212 & 0.41972 & 1.37705 \\
\hline $\mathrm{H}$ & 1.79406 & 0.60812 & -2.45202 \\
\hline $\mathrm{H}$ & -2.94769 & -0.64975 & -1.45176 \\
\hline $\mathrm{H}$ & -2.82453 & 1.32073 & 2.21723 \\
\hline
\end{tabular}

$4 a-d i h y-t s-c$ 
Supporting Information for Donoghue et al.: Ligand and Substrate Effects on the Mechanisms of the Rhodium...

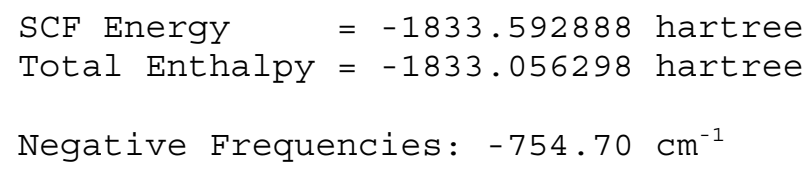

\begin{tabular}{|c|c|c|c|}
\hline $\mathrm{Rh}$ & 0.02270 & -0.84748 & 0.34953 \\
\hline 0 & -1.20164 & -2.62449 & 1.04593 \\
\hline $\mathrm{C}$ & -0.67369 & -3.73158 & 0.86604 \\
\hline $\mathrm{N}$ & 0.44084 & -3.89874 & 0.13585 \\
\hline $\mathrm{C}$ & 1.01709 & -2.76938 & -0.58163 \\
\hline $\mathrm{C}$ & 0.17023 & -2.10867 & -1.53755 \\
\hline $\mathrm{C}$ & 2.41295 & -3.00056 & -0.91175 \\
\hline $\mathrm{N}$ & 3.51814 & -3.22387 & -1.18781 \\
\hline $\mathrm{H}$ & -0.79991 & -2.55349 & -1.72484 \\
\hline $\mathrm{H}$ & 0.64189 & -1.61353 & -2.37615 \\
\hline $\mathrm{H}$ & 0.86407 & -4.81328 & 0.03577 \\
\hline $\mathrm{H}$ & -1.09323 & -4.64209 & 1.30976 \\
\hline $\mathrm{P}$ & 1.38040 & 0.99829 & 0.23751 \\
\hline $\mathrm{P}$ & -1.76782 & 0.61701 & -0.07692 \\
\hline $\mathrm{H}$ & 0.04643 & -0.32675 & 1.84755 \\
\hline $\mathrm{H}$ & 1.30987 & -1.91683 & 0.60615 \\
\hline C & 2.26646 & 1.44850 & -1.35597 \\
\hline $\mathrm{C}$ & 2.78142 & 1.09958 & 1.46802 \\
\hline C & -2.90333 & 0.32837 & -1.53256 \\
\hline C & -2.98336 & 0.82014 & 1.33294 \\
\hline C & 0.25279 & 2.44751 & 0.55254 \\
\hline $\mathrm{H}$ & 0.75951 & 3.39381 & 0.34339 \\
\hline $\mathrm{H}$ & 0.01172 & 2.43503 & 1.62015 \\
\hline $\mathrm{C}$ & -1.02371 & 2.31521 & -0.29307 \\
\hline $\mathrm{H}$ & -1.74518 & 3.09243 & -0.02433 \\
\hline $\mathrm{H}$ & -0.79419 & 2.44890 & -1.35521 \\
\hline $\mathrm{C}$ & -4.17457 & 1.68873 & 0.86650 \\
\hline $\mathrm{H}$ & -4.90483 & 1.74817 & 1.67987 \\
\hline $\mathrm{H}$ & -3.85492 & 2.71711 & 0.63337 \\
\hline $\mathrm{C}$ & -4.14913 & 1.24030 & -1.45710 \\
\hline $\mathrm{H}$ & -4.84707 & 0.94698 & -2.24805 \\
\hline $\mathrm{H}$ & -3.86068 & 2.28916 & -1.64132 \\
\hline 0 & -4.85753 & 1.12588 & -0.23926 \\
\hline $\mathrm{C}$ & 3.17447 & 2.67797 & -1.09715 \\
\hline $\mathrm{H}$ & 3.70661 & 2.91602 & -2.02390 \\
\hline $\mathrm{H}$ & 2.58595 & 3.56580 & -0.81851 \\
\hline $\mathrm{C}$ & 3.66144 & 2.34495 & 1.19383 \\
\hline $\mathrm{H}$ & 4.53455 & 2.29987 & 1.85187 \\
\hline $\mathrm{H}$ & 3.10505 & 3.26353 & 1.44472 \\
\hline 0 & 4.16583 & 2.41400 & -0.12548 \\
\hline $\mathrm{C}$ & 3.09610 & 0.29988 & -1.94768 \\
\hline $\mathrm{H}$ & 2.48763 & -0.57019 & -2.19156 \\
\hline $\mathrm{H}$ & 3.89423 & -0.01296 & -1.27102 \\
\hline $\mathrm{H}$ & 3.57191 & 0.63711 & -2.87441 \\
\hline $\mathrm{C}$ & 2.34464 & 1.05531 & 2.93700 \\
\hline $\mathrm{H}$ & 1.75968 & 0.15973 & 3.15734 \\
\hline $\mathrm{H}$ & 1.74912 & 1.93154 & 3.21491 \\
\hline $\mathrm{H}$ & 3.23017 & 1.04546 & 3.58095 \\
\hline $\mathrm{C}$ & -2.20469 & 0.44696 & -2.89246 \\
\hline $\mathrm{H}$ & -1.30181 & -0.16688 & -2.93858 \\
\hline $\mathrm{H}$ & -1.92665 & 1.48166 & -3.1200 \\
\hline
\end{tabular}


Supporting Information for Donoghue et al.: Ligand and Substrate Effects on the Mechanisms of the Rhodium...

$\begin{array}{rrrr}\mathrm{H} & -2.87698 & 0.11139 & -3.68862 \\ \mathrm{C} & -3.46518 & -0.52255 & 1.90402 \\ \mathrm{H} & -2.63567 & -1.14315 & 2.24285 \\ \mathrm{H} & -4.03924 & -1.08415 & 1.16208 \\ \mathrm{H} & -4.12677 & -0.33385 & 2.75605 \\ \mathrm{H} & 1.48581 & 1.73974 & -2.07081 \\ \mathrm{H} & -2.43729 & 1.36758 & 2.11285 \\ \mathrm{H} & -3.24524 & -0.70505 & -1.39299 \\ \mathrm{H} & 3.38987 & 0.21280 & 1.24935\end{array}$

$4 a-a l h y-A$

$\begin{array}{ll}\text { SCF Energy } & =-1833.657954 \text { hartree } \\ \text { Total Enthalpy } & =-1833.115283 \text { hartree }\end{array}$

$\begin{array}{rrrr}\mathrm{Rh} & 0.87120 & -0.14023 & -0.80148 \\ \mathrm{O} & 0.76870 & -0.40298 & -2.94282 \\ \mathrm{C} & 1.90044 & -0.46854 & -3.47779 \\ \mathrm{~N} & 3.02107 & -0.43169 & -2.76759 \\ \mathrm{C} & 2.98357 & -0.46264 & -1.29950 \\ \mathrm{C} & 4.23350 & 0.18726 & -0.73358 \\ \mathrm{C} & 2.66819 & -1.80421 & -0.84139 \\ \mathrm{~N} & 2.15704 & -2.79336 & -0.47113 \\ \mathrm{H} & 5.14282 & -0.22700 & -1.19258 \\ \mathrm{H} & 4.31320 & 0.01443 & 0.33880 \\ \mathrm{H} & 3.91315 & -0.52228 & -3.24521 \\ \mathrm{H} & 1.99398 & -0.55800 & -4.56484 \\ \mathrm{P} & 0.79413 & 0.28020 & 1.48390 \\ \mathrm{P} & -1.52582 & -0.12057 & -0.67569 \\ \mathrm{H} & 0.92662 & 1.37263 & -0.95038 \\ \mathrm{H} & 4.22060 & 1.26449 & -0.91597 \\ \mathrm{C} & 1.83662 & 1.57382 & 2.35086 \\ \mathrm{C} & 1.07346 & -1.26015 & 2.52645 \\ \mathrm{C} & -2.31528 & 1.26094 & -1.67081 \\ \mathrm{C} & -2.46761 & -1.62169 & -1.27120 \\ \mathrm{H} & 0.87657 & -2.11204 & 1.86584 \\ \mathrm{H} & -1.60630 & 2.09726 & -1.64667 \\ \mathrm{H} & 1.21854 & 1.85471 & 3.21429 \\ \mathrm{H} & -3.47814 & -1.51696 & -0.85399 \\ \mathrm{C} & -0.92944 & 0.89797 & 1.84309 \\ \mathrm{H} & -0.95361 & 1.94191 & 1.50896 \\ \mathrm{H} & -1.09737 & 0.90341 & 2.92426 \\ \mathrm{C} & -1.99387 & 0.07331 & 1.11246 \\ \mathrm{H} & -2.05400 & -0.93745 & 1.53159 \\ \mathrm{H} & -2.98617 & 0.52099 & 1.21607 \\ \mathrm{C} & 2.56787 & -1.30754 & 2.91590 \\ \mathrm{H} & 2.73874 & -2.18750 & 3.54358 \\ \mathrm{H} & 3.20336 & -1.41031 & 2.02403 \\ \mathrm{C} & 3.14634 & 0.98984 & 2.92024 \\ \mathrm{H} & 3.86824 & 0.80979 & 2.11054 \\ \mathrm{H} & 3.59008 & 1.73021 & 3.59337 \\ \mathrm{C} & -2.59957 & -1.57171 & -2.80835 \\ \mathrm{H} & -3.23863 & -2.39951 & -3.13175 \\ \mathrm{H} & -1.61220 & -1.70056 & -3.27768 \\ \mathrm{C} & -2.40873 & 0.76996 & -3.13688 \\ \mathrm{H} & -1.40662 & 0.58510 & -3.54695 \\ \mathrm{H} & -2.88702 & 1.55098 & -3.73668\end{array}$


Supporting Information for Donoghue et al.: Ligand and Substrate Effects on the Mechanisms of the Rhodium...

$\begin{array}{rrrr}\mathrm{C} & 0.18441 & -1.34748 & 3.77796 \\ \mathrm{H} & -0.88260 & -1.36474 & 3.54087 \\ \mathrm{H} & 0.38060 & -0.52306 & 4.46877 \\ \mathrm{H} & 0.41172 & -2.27801 & 4.30935 \\ \mathrm{C} & 2.09456 & 2.81932 & 1.49328 \\ \mathrm{H} & 1.16437 & 3.29035 & 1.16165 \\ \mathrm{H} & 2.68260 & 2.57707 & 0.60337 \\ \mathrm{H} & 2.64815 & 3.56522 & 2.07378 \\ \mathrm{C} & -1.85024 & -2.94375 & -0.79552 \\ \mathrm{H} & -1.82167 & -3.01714 & 0.29627 \\ \mathrm{H} & -0.82890 & -3.07476 & -1.16709 \\ \mathrm{H} & -2.45068 & -3.78543 & -1.15742 \\ \mathrm{C} & -3.68266 & 1.72598 & -1.14755 \\ \mathrm{H} & -3.62462 & 2.15239 & -0.14205 \\ \mathrm{H} & -4.41295 & 0.91245 & -1.14585 \\ \mathrm{H} & -4.07141 & 2.50932 & -1.80756 \\ \mathrm{O} & 2.96151 & -0.18396 & 3.68684 \\ \mathrm{O} & -3.22218 & -0.38476 & -3.27072\end{array}$

$4 a-a l h y-C$

SCF Energy $\quad=-1833.637675$ hartree

Total Enthalpy $=-1833.094770$ hartree

$\begin{array}{rrrr}\mathrm{Rh} & 0.75288 & 0.04808 & -0.60139 \\ \mathrm{O} & 0.73015 & -0.01000 & -2.77761 \\ \mathrm{C} & 1.71465 & 0.11301 & -3.53656 \\ \mathrm{~N} & 2.98629 & -0.08848 & -3.20812 \\ \mathrm{C} & 3.44197 & -0.57818 & -1.88765 \\ \mathrm{C} & 2.85428 & 0.21049 & -0.70093 \\ \mathrm{C} & 4.91316 & -0.51255 & -1.89262 \\ \mathrm{~N} & 6.07170 & -0.45289 & -1.86978 \\ \mathrm{H} & 3.13262 & 1.26944 & -0.80130 \\ \mathrm{H} & 3.34826 & -0.17649 & 0.19152 \\ \mathrm{H} & 3.68111 & 0.01991 & -3.93708 \\ \mathrm{H} & 1.55161 & 0.40961 & -4.58029 \\ \mathrm{P} & -1.67962 & -0.32699 & -0.51460 \\ \mathrm{P} & 0.60474 & 0.36953 & 1.65924 \\ \mathrm{H} & 0.99874 & -1.39225 & -0.22340 \\ \mathrm{H} & 3.16371 & -1.63685 & -1.80074 \\ \mathrm{C} & -2.30028 & -1.99576 & -1.09086 \\ \mathrm{C} & -2.82046 & 0.79913 & -1.49257 \\ \mathrm{C} & 1.94770 & -0.25475 & 2.81128 \\ \mathrm{C} & 0.46215 & 2.17947 & 2.13429 \\ \mathrm{H} & -2.38130 & 1.80384 & -1.43522 \\ \mathrm{H} & 2.36361 & -1.14758 & 2.32873 \\ \mathrm{H} & -0.09284 & 2.19103 & 3.08148 \\ \mathrm{H} & -3.32913 & -2.08593 & -0.71991 \\ \mathrm{C} & -0.97660 & -0.44814 & 2.22303 \\ \mathrm{H} & -1.20780 & -0.13707 & 3.24686 \\ \mathrm{H} & -0.76071 & -1.52203 & 2.25433 \\ \mathrm{C} & -2.16469 & -0.16969 & 1.28547 \\ \mathrm{H} & -2.99842 & -0.83972 & 1.51980 \\ \mathrm{H} & -2.53318 & 0.84988 & 1.43661 \\ \mathrm{C} & 3.06888 & 0.80740 & 2.90934 \\ \mathrm{H} & 3.81203 & 0.46384 & 3.63539 \\ \mathrm{H} & 3.57986 & 0.94357 & 1.94623\end{array}$


Supporting Information for Donoghue et al.: Ligand and Substrate Effects on the Mechanisms of the Rhodium...

$\begin{array}{rrrr}\mathrm{C} & 1.85988 & 2.77391 & 2.41629 \\ \mathrm{H} & 2.43444 & 2.85310 & 1.47800 \\ \mathrm{H} & 1.73201 & 3.78638 & 2.81238 \\ \mathrm{C} & -2.36776 & -1.98435 & -2.63442 \\ \mathrm{H} & -2.77657 & -2.94060 & -2.97660 \\ \mathrm{H} & -1.35502 & -1.87835 & -3.05576 \\ \mathrm{C} & -2.74367 & 0.33899 & -2.96833 \\ \mathrm{H} & -1.71449 & 0.42647 & -3.34589 \\ \mathrm{H} & -3.38893 & 0.98204 & -3.57531 \\ \mathrm{C} & 1.43454 & -0.62084 & 4.21574 \\ \mathrm{H} & 0.68002 & -1.41041 & 4.19842 \\ \mathrm{H} & 1.02460 & 0.25160 & 4.73160 \\ \mathrm{H} & 2.27449 & -0.98699 & 4.81559 \\ \mathrm{C} & -0.27740 & 3.03041 & 1.09131 \\ \mathrm{H} & -1.31261 & 2.71299 & 0.94693 \\ \mathrm{H} & 0.22792 & 3.00186 & 0.11835 \\ \mathrm{H} & -0.30209 & 4.07710 & 1.41348 \\ \mathrm{C} & -1.47146 & -3.17732 & -0.57398 \\ \mathrm{H} & -1.44940 & -3.21803 & 0.51959 \\ \mathrm{H} & -0.43815 & -3.13029 & -0.93266 \\ \mathrm{H} & -1.90486 & -4.12055 & -0.92340 \\ \mathrm{C} & -4.27430 & 0.84353 & -0.99576 \\ \mathrm{H} & -4.35488 & 1.16176 & 0.04724 \\ \mathrm{H} & -4.76134 & -0.12846 & -1.10396 \\ \mathrm{H} & -4.84213 & 1.56041 & -1.59929 \\ \mathrm{O} & 2.59118 & 2.05319 & 3.38652 \\ \mathrm{O} & -3.22835 & -0.98262 & -3.14839\end{array}$

\section{References}

4 (b) Feldgus, S.; Landis, C. R. J. Am. Chem. Soc. 2000, 122, 12714. 